\title{
Nondestructive evaluation of fiber reinforced polymer bridge decks using ground penetrating radar and infrared thermography
}

Cheng Lok Hing

West Virginia University

Follow this and additional works at: https://researchrepository.wvu.edu/etd

\section{Recommended Citation}

Hing, Cheng Lok, "Nondestructive evaluation of fiber reinforced polymer bridge decks using ground penetrating radar and infrared thermography" (2006). Graduate Theses, Dissertations, and Problem Reports. 2715.

https://researchrepository.wvu.edu/etd/2715

This Dissertation is protected by copyright and/or related rights. It has been brought to you by the The Research Repository @ WVU with permission from the rights-holder(s). You are free to use this Dissertation in any way that is permitted by the copyright and related rights legislation that applies to your use. For other uses you must obtain permission from the rights-holder(s) directly, unless additional rights are indicated by a Creative Commons license in the record and/ or on the work itself. This Dissertation has been accepted for inclusion in WVU Graduate Theses, Dissertations, and Problem Reports collection by an authorized administrator of The Research Repository @ WVU.

For more information, please contact researchrepository@mail.wvu.edu. 


\title{
Nondestructive Evaluation of Fiber Reinforced Polymer Bridge Decks Using Ground Penetrating Radar and Infrared Thermography \\ by
}

\section{Cheng Lok Hing}

\author{
A DISSERTATION \\ Submitted to the \\ College of Engineering and Mineral Resources \\ West Virginia University \\ in partial fulfillment of the requirements \\ for the degree of \\ Doctor of Philosophy \\ in \\ Civil and Environmental Engineering
}

\author{
Udaya B. Halabe, Ph.D., P.E., Chair \\ Hota V. GangaRao, Ph.D., P.E. \\ Powsiri Klinkhachorn, Ph.D. \\ Ruifeng Liang, Ph.D. \\ Hema J. Siriwardane, Ph.D., P.E. \\ West Virginia University \\ Morgantown, West Virginia \\ 2006
}

Keywords: Ground Penetrating Radar, GPR, Infrared Thermography, IRT, Fiber Reinforced Polymer, FRP, Nondestructive Evaluation, NDE 


\section{ABSTRACT \\ Nondestructive Evaluation of Fiber Reinforced Polymer Bridge Decks \\ Using Ground Penetrating Radar and Infrared Thermography}

Cheng Lok Hing

Recent studies have focused on the development of Fiber Reinforcement Polymer (FRP) as an alternative construction material for highway bridge decks.

The goal of this study was to explore the viability of nondestructive testing of FRP bridge decks using infrared thermography (IRT) and ground penetrating radar (GPR).

All tests were conducted on a 6' x 3' (1828.8mm x 914.4mm) low profile (4" or 101.6mm deep) FRP bridge deck and a 3' x 2' (914.4mm x 609.6mm) low profile FRP bridge deck specimen with embedded delaminations. Replaceable wearing surface modules with air-voids of varying sizes were used to simulate air-filled debonds between the wearing surface and the FRP bridge deck. To simulate the water-filled debonds, custom made water-pouches were placed in the air-voids.

Solar radiation, commercially available heater, and heating blankets were utilized as active heat sources in the IRT tests. The effectiveness of each heat source in subsurface detection of defects was examined.

A simple finite element model was created to study the heat transfer phenomena between the FRP bridge deck with wearing surface and the surroundings. The FE model enabled a theoretical study of the effect of subsurface defect thickness on the surface temperature profile. Results from the model were also compared to the experimental results obtained through the IRT tests.

A $1.5 \mathrm{GHz}$ ground-coupled antenna and a $2.0 \mathrm{GHz}$ air-coupled antenna were utilized in the GPR tests for this study. They were used in an attempt to identify both airfilled and water-filled debonds and delaminations. The effectiveness of each antenna in detecting subsurface defects was carefully examined.

The results of this study have shown that a combination of GPR and IRT techniques can lead to an effective nondestructive testing system for detecting subsurface defects in FRP Bridge Decks. 


\section{ACKNOWLEDGEMENTS}

I would like to extend my utmost appreciation to Dr. Udaya B. Halabe, my academic and thesis advisor, as well as my mentor, for his kindness, encouragement, and guidance through the entire journey. I would like to thank Dr. Hota V. GangaRao, Dr. Powsiri Klinkhachorn, Dr. Ruifeng Liang, and Dr. Hema J. Siriwardane for serving in the Ph.D. Advisory and Examining Committee (AEC) and for their time and effort in reviewing this dissertation.

There are a number of people who had helped me in making this a successful study. The assistance provided by Jerry Nestor and Bill Comstock, laboratory technicians, are greatly appreciated. Thanks to Bhyrav Mutnuri for his help in determining the FRP's conductivity value. Without the help of Shashanka Dutta, Sandeep Pyakurel, Manjunath Dasarath Rao, and Scott Mercer, this would have been a tall task to fill. I would also like to thank Dr. Roger C. Viadero for his help in providing some of the supplies necessary for the completion of this study.

I would like to thank my parents and all my family members for their constant encouragement and support. Last but not the least, I would like to thank all my friends for their help directly or indirectly in my research. They have made my stay at Morgantown some of the best times of my life.

I would like to gratefully acknowledge the funding for this research provided by the Federal Highway Administration (FHWA) under the CFC - FHWA Designated Center of Excellence program. 


\section{DEDICATIONS}

This dissertation is dedicated to my wife Amanda Lee, who had stood by me through thick and thin; my parents Kong Hock Hing, Kong Khim Leong; my brothers Teik Chong Hing, Teik Sia Hing, Teik Khun Hing; my sisters Siew Eng Hing, Mee Lang Hing, Amy Hing, Be Leng Hing, and Mee Lim Hing. 


\section{TABLE OF CONTENTS}

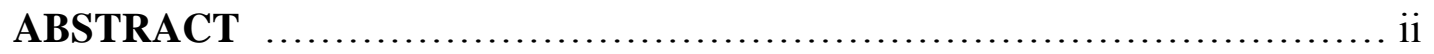

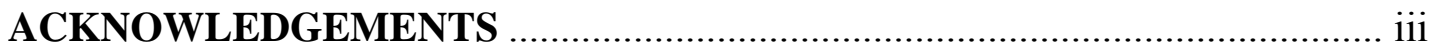

DEDICATION ................................................................. iv

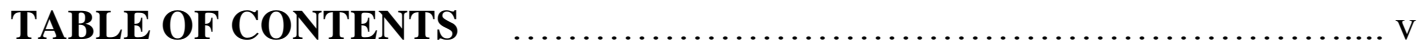

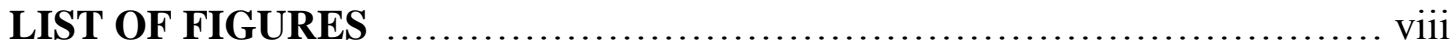

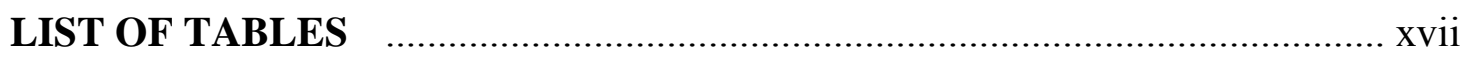

CHAPTER 1 - INTRODUCTION …................................. 1

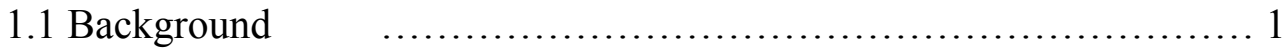

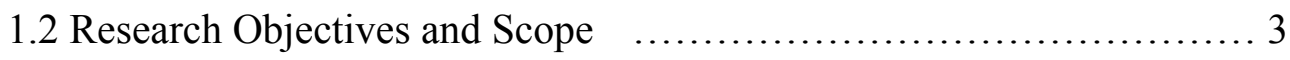

1.3 Organization $\quad$...................................................... 5

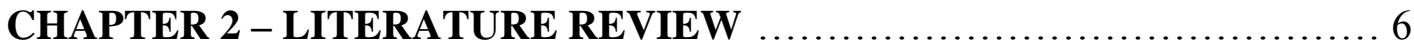

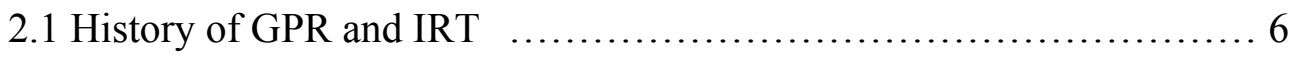

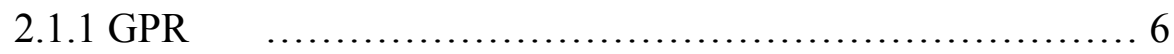

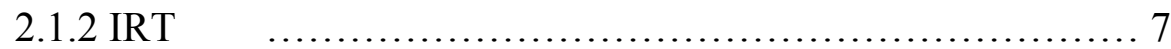

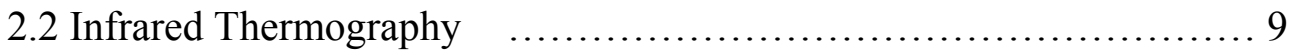

2.3 Ground Penetrating Radar $\quad$........................................ 10

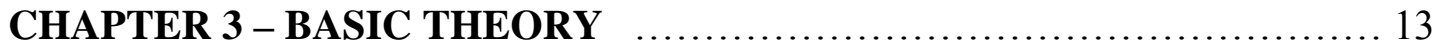

3.1 Basics of Ground Penetrating Radar (GPR) $\quad$.................... 13

3.1.1 Electromagnetic Properties and Radar

Wave Propagation $\quad$.................................... 14

3.1.2 Detection of Voids (................................... 19

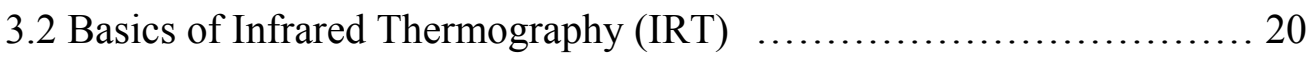

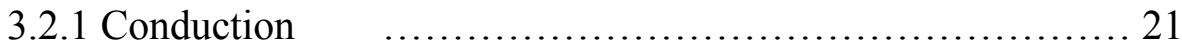

3.2.2 Convection $\quad$.......................................... 21

3.2.3 Radiation $\quad$......................................... 23

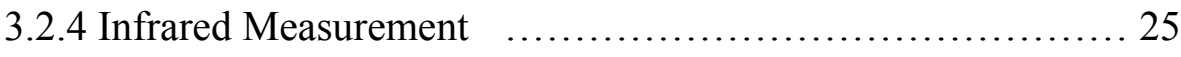




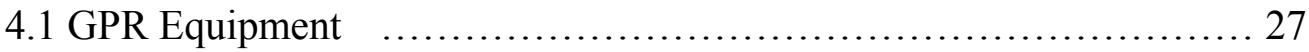

4.2 Infrared Equipment $\quad$......................................... 31

4.2 .1 Heating Sources ........................................... 32

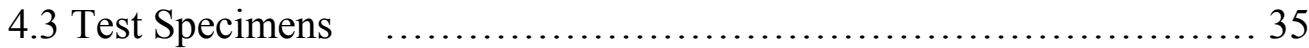

4.4 Preparation of Defects $\quad$........................................ 38

CHAPTER 5 - EXPERIMENTAL RESULTS FROM INFRARED

THERMOGRAPHY ...................................... 45

5.1 IRT Using Commercially Available Heater As

Active Heat Source f.......................................... 45

5.2 IRT Using Solar Radiation as Active Heat Source..................... 48

5.2.1 Solar Radiation Test Conducted in September 2005 ........ 48

5.2.2 Solar Radiation Test Conducted in March 2006............... 57

5.3 IRT Using Heating Blanket as Active Heat Source $\quad$................. 61

5.4 Conclusions $\quad$................................................. 72

CHAPTER 6 - FINITE ELEMENT MODELING …................. 74

6.1 Thermographic Defect Recognition and

Measurement Theory …....................................... 75

6.2 Thermal Properties $\quad$...................................... 76

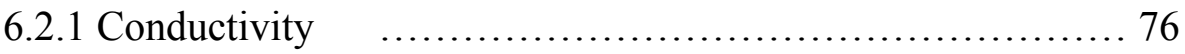

6.2.2 Emissivity $\quad$........................................ 80

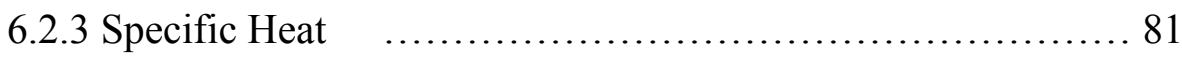

6.3 FE Model f.................................................... 85

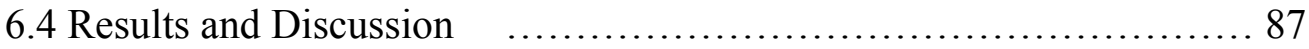

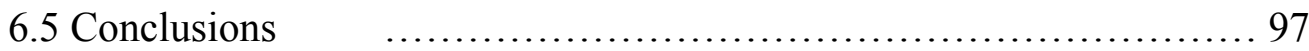

CHAPTER 7 - EXPERIMENTAL RESULTS FROM

GROUND PENETRATING RADAR _.............. 98

7.1 Full Scale GPR Test $\quad$......................................... 101

7.1.1 Detection of Water-filled and Air-filled Debonds $\quad$......... 101

7.1.2 Delamination Detection Using GPR .................... 134

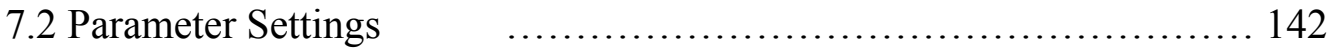


7.2.1 Record Length (512 Points versus 1024 Points)............... 142

7.2.2 Constant Gain versus Linear Gain $\quad$...................... 143

7.3 Blind Test ................................................... 144

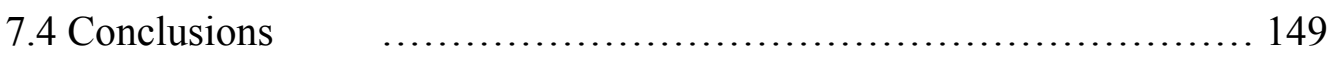

CHAPTER 8 - CONCLUSIONS AND RECOMMENDATIONS $\quad$........ 150

8.1 Conclusions $\quad$................................................ 150

8.2 Recommendations ............................................... 153

REFERENCES

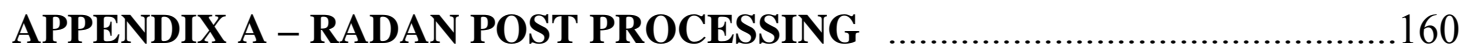

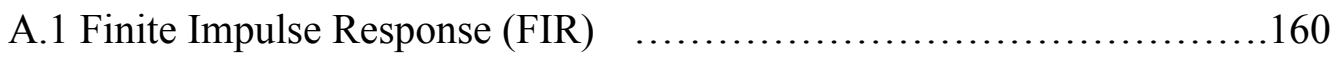

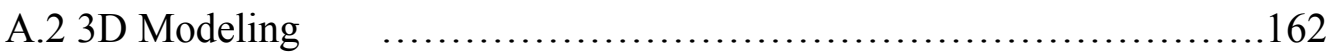




\section{LIST OF FIGURES}

Figure 2.1: Multi-component imaging results................................ 12

Figure 3.1: Electromagnetic spectrum showing the infrared measurement region...... 25

Figure 4.1(a): GPR system with $2.0 \mathrm{GHz}$ horn antenna on the modified push-cart ...... 28

Figure 4.1(b): Hand-held 1.5 GHz ground-coupled antenna and survey wheel

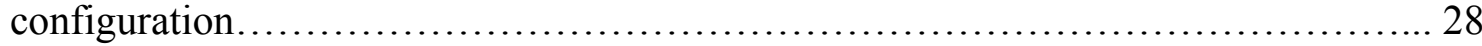

Figure 4.2(a): Footprint of the $2.0 \mathrm{GHz}$ air-coupled horn antenna................... 29

Figure 4.2(b): Footprint of the $1.5 \mathrm{GHz}$ ground-coupled antenna................... 30

Figure 4.3 ThermaCAM ${ }^{\mathrm{TM}} \mathrm{S} 60$ infrared camera from FLIR Systems................ 31

Figure 4.4: Heating blanket with external temperature control box................. 35

Figure 4.5: Cross-section of low-profile FRP bridge deck........................ 36

Figure 4.6 Schematic Diagram of Pultrusion Process........................... 37

Figure 4.7: Photographs showing manufacturing of FRP bridge deck in a factory using Pultrusion process................................................... 38

Figure 4.8: wearing surfaces module of dimensions 36 " x 24" x 3/8" (914.4mm x

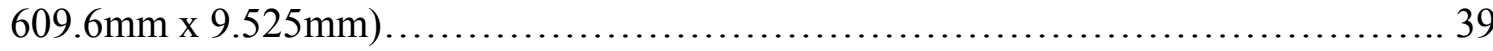

Figure 4.9: Pouch made using Press'N Seal Glad wrap.......................... 40

Figure 4.10: Photograph showing water-filled pouches........................ 41

Figure 4.11: Glad pouch being filled with water using syringe.................... 41

Figure 4.12: Bottom side of a wearing surface with two water-filled simulated

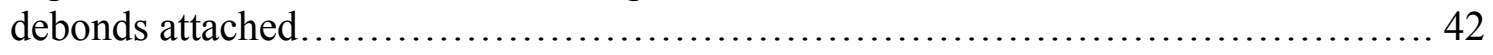

Figure 4.13: The machine drilling out voids to be used as delamination............... 43

Figure 4.14: Cutting out the filling channel................................ 43

Figure 4.15: Two filling channels were cut out by the machine.................. 44

Figure 4.16: The two FRP modules to be joined together........................ 44 
Figure 5.1: Infrared image of 4" x 4" x 0.09" (101.6mm x 101.6mm x 2.25mm) air-filled debond........................................................ 46

Figure 5.2: Debonds detected using infrared image........................... 47

Figure 5.3: Defects map of delamination module for IRT solar radiation experiment.. 48 Figure 5.4: Defects map of debond module for IRT solar radiation experiment........ 49

Figure 5.5: The bridge deck module with wearing surfaces.................... 50

Figure 5.6: The setup of the solar radiation IRT test........................... 50

Figure 5.7: IRT image of FRP module with delaminations $\ldots \ldots \ldots \ldots \ldots \ldots \ldots \ldots \ldots .51$

Figure 5.8: IRT image \#1 of the bridge deck at 11:30 AM...................... 52

Figure 5.9: IRT image \#2 of the bridge deck at 11:30 AM..................... 52

Figure 5.10: IRT image taken after 5 hours of solar heating at 2:30 PM............ 53

Figure 5.11: Surface temperature of defects versus defect-free area on wearing

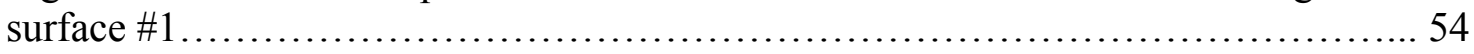

Figure 5.12: Surface temperature of defects versus defect-free area on wearing

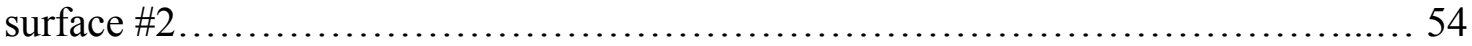

Figure 5.13: Temperature differences of various defects on wearing surface \#1 $\ldots . . .56$

Figure 5.14(a): Temperature differences of various defects on all wearing surfaces.... 56

Figure 5.14(b): Temperature differences of various defects on all wearing

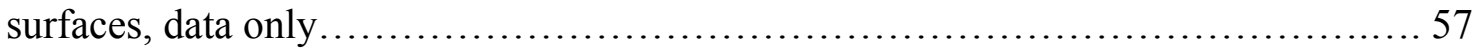

Figure 5.15: FRP bridge deck after 30 minutes of solar heating................. 58

Figure 5.16: FRP bridge deck after 30 minutes of solar heating $\ldots \ldots \ldots \ldots \ldots \ldots \ldots \ldots 58$

Figure 5.17: FRP bridge deck after 3 hours of solar heating..................... 59

Figure 5.18: Defects map of debond module for IRT solar radiation experiment...... 60

Figure 5.19: The experimental setup for IRT test using heating blanket as active heat source.

Figure 5.20: The experimental setup for IRT test using heating blanket as active heat source. 
Figure 5.21(a): Defects map for IRT using heating blanket as active heat source..... 63

Figure 5.21(b): Defects map for IRT using heating blanket as active heat source...... 64

Figure 5.22: Results of IRT on air-filled defects using heating blanket as active heat source.

Figure 5.23: Results of IRT on air-filled defects using heating blanket as active heat source

Figure 5.24: Results of IRT on water-filled defects using heating blanket as active heat source.

Figure 5.25: Results of IRT on water-filled defects using heating blanket as active heat source.

Figure 5.26: Results of IRT on smaller size defects using heating blanket as active heat source.

Figure 5.27: Results of IRT on smaller size defects using heating blanket as active heat source.

Figure 5.28: Temperature profile of various air-filled debonds

Figure 5.29: Temperature profile of defective minus defect-free for various air-filled debonds.

Figure 5.30: Temperature profile of various air-filled debonds.

Figure 5.31: Temperature profile of defective minus defect-free for various air-filled debonds.

Figure 5.32: Temperature profile of defective minus defect-free for 12 " x 12 " x 0.03 " water-filled debonds.......................................................... 71

Figure 5.33: Temperature profile of defective minus defect-free for 8 " x 8 " x 0.09 " water-filled debonds................................................... 71

Figure 6.1: Heat flow due to a flash pulse. Initially the pulse causes thin layer of uniform heat on the surface (Left). Heat flows into the sample (Center). When a defect is encountered, the heat is trapped between the defect and the sample surface, giving rise to lateral heat flow above the defect........................ 76

Figure 6.2: Experimental set up of Unitherm $2022 \ldots \ldots \ldots \ldots \ldots \ldots \ldots \ldots \ldots \ldots \ldots \ldots$ 


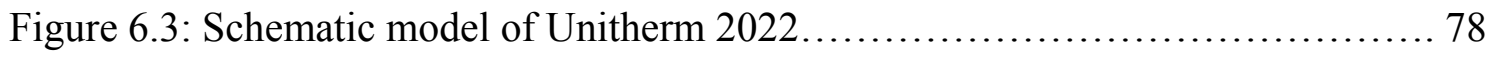

Figure 6.4(a): Circular Transpo wearing surface disc samples....................... 78

Figure 6.4(b): Circular FRP disc samples...................................... 79

Figure 6.5 : The digital deep fryer............................................. 82

Figure 6.6: Weighing of the sample using a digital scale........................... 82

Figure 6.7: Triangular solid mesh of the FE model.............................. 86

Figure 6.8: FE mesh of wearing surface layer with embedded debond .................. 87

Figure 6.9: Temperature profile for a $0.1 \mathrm{~m} \times 0.1 \mathrm{~m}$ x $0.0025 \mathrm{~m}$ air-filled debond

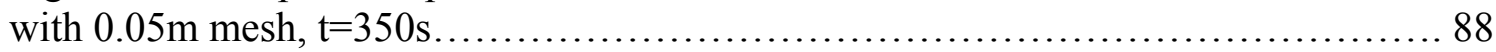

Figure 6.10: Temperature profile for a $0.1 \mathrm{~m} \times 0.1 \mathrm{~m} \times 0.0025 \mathrm{~m}$ air-filled debond

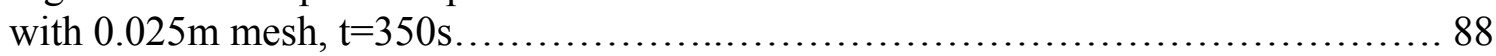

Figure 6.11: Temperature profile for a $0.1 \mathrm{~m} \times 0.1 \mathrm{~m} \times 0.0025 \mathrm{~m}$ air-filled debond

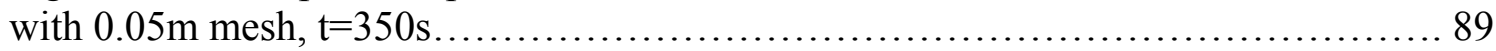

Figure 6.12: Temperature profile for a $0.1 \mathrm{~m}$ x $0.1 \mathrm{~m}$ x $0.0025 \mathrm{~m}$ air-filled debond with $0.025 \mathrm{~m}$ mesh, $\mathrm{t}=350 \mathrm{~s}$.

Figure 6.13: Temperature profile for a $0.1 \mathrm{~m} \times 0.1 \mathrm{~m} \times 0.0015 \mathrm{~m}$ air-filled debond

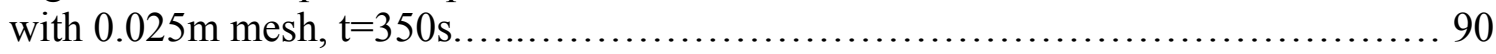

Figure 6.14: Temperature profile for a $0.1 \mathrm{~m}$ x $0.1 \mathrm{~m}$ x $0.0015 \mathrm{~m}$ air-filled debond with $0.025 \mathrm{~m}$ mesh, $\mathrm{t}=350 \mathrm{~s}$.

Figure $6.15(\mathrm{a})$ : Temperature profile of various $0.1 \mathrm{~m} \times 0.1 \mathrm{~m}$ air-filled debonds minus defect-free.

Figure $6.15(\mathrm{~b})$ : Temperature profile of various $0.1 \mathrm{~m} \times 0.1 \mathrm{~m}$ air-filled debonds minus defect-free (after removal of heat, 200 seconds comparison).

Figure 6.16: Temperature profile of various $0.05 \mathrm{~m} \times 0.05 \mathrm{~m}$ air-filled debonds minus defect-free.

Figure 6.17: Temperature profile for a $0.1 \mathrm{~m} \times 0.1 \mathrm{~m} \times 0.0025 \mathrm{~m}$ water-filled debond with $0.025 \mathrm{~m}$ mesh, $\mathrm{t}=350 \mathrm{~s}$......

Figure 6.18: Temperature profile for a $0.1 \mathrm{~m} \times 0.1 \mathrm{~m} \times 0.0025 \mathrm{~m}$ water-filled debond with $0.025 \mathrm{~m}$ mesh, $\mathrm{t}=650 \mathrm{~s}$. 
Figure 6.19: Temperature profile of defective minus defect-free for $0.1 \mathrm{~m} \times 0.1 \mathrm{~m}$ water-filled debonds............................................................... 95

Figure 6.20: Temperature profile of defective minus defect-free for $0.1 \mathrm{~m} \times 0.1 \mathrm{~m}$ partially water-filled debonds..................................................... 97

Figure 7.1: The setup of the preliminary GPR test................................ 98

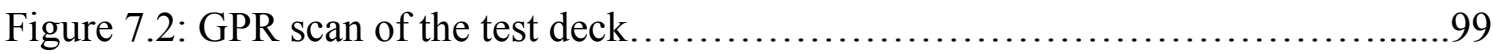

Figure 7.3: Processed signals as Excel plot........................................ 100

Figure 7.4: Result of subtraction of defect-free signal from water-filled signal......... 101

Figure 7.5: FRP Deck module with the wooden platform setup...................... 102

Figure 7.6: Hardware configuration of SIR-20 GPR system with $2.0 \mathrm{GHz}$ horn

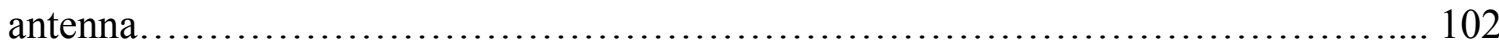

Figure 7.7: 1.5 $\mathrm{GHz}$ ground-coupled antenna................................... 103

Figure 7.8: A typical GPR scan of an FRP deck using 1.5GHz ground-coupled antenna

Figure 7.9: A typical GPR scan of an FRP deck using 2.0GHz air-coupled horn antenna.

Figure 7.10: 3D profile created by collecting three passes of GPR data at equal distances (9") apart using ground-coupled antenna................................ 107

Figure 7.11: GPR processed data after FIR filter was applied to the raw data in Figure 7.8

Figure 7.12: 4" x 4" x 0.09" (101.6mm x 101.6mm x 2.25mm) water-filled debond shown in $3 \mathrm{D}$ profile using ground-coupled antenna. 108

Figure 7.13: 4" x 4" x 0.09" (101.6mm x 101.6mm x 2.25mm) air-filled debond in the central region of the FRP deck is not visible in this 3D profile obtained using ground-coupled antenna................................................ 109

Figure 7.14: 3D profile generated by the air-coupled horn antenna................... 109

Figure 7.15: 4" x 4" x 0.03" (101.6mm x 101.6mm x 0.75mm) water-filled debond seen in a 2D GPR scan obtained using ground-coupled antenna. 
Figure 7.16: 2" x 2" x 0.06" (50.8mm x 50.8mm x 1.5mm) water-filled debond...... 112

Figure 7.17: Comparison of signal amplitudes for GPR waveforms (ground-coupled antenna) from various water-filled debonds...................... 114

Figure 7.18: Waveforms corresponding to various debonds after subtraction

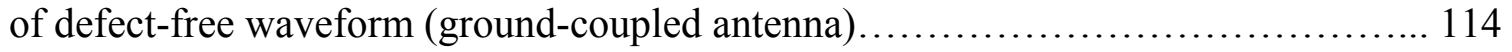

Figure 7.19: Comparison of signal amplitudes for GPR waveforms (air-coupled horn antenna) from various water-filled debonds.................... 115

Figure 7.20: Layout of defects.......................................... 117

Figure 7.21: 2D image of an air-filled debond scan............................ 118

Figure 7.22: Signals corresponding to the original air-filled defects data after subtraction of defect-free waveform (horn antenna).

Figure 7.23: Waveforms corresponding to various water-filled debonds after subtraction of defect-free waveform (ground-coupled antenna)........................... 119

Figure 7.24: Signals corresponding to the air-filled defects data, after subtraction of defect-free waveform, with FIR (horn antenna).............................. 120

Figure 7.25: 2D image of a water-filled debond scan.......................... 121

Figure 7.26: 2D image of a water-filled debond scan with FIR.................... 121

Figure 7.27: $2 \mathrm{D}$ image of the original scan for the 12 " $\mathrm{x} 12$ " $\mathrm{x} 0.06$ "

water-filled defect.................................................... 122

Figure 7.28: 2D image of the FIR scan for the 12 " x 12 " x 0.06 "

water-filled defect................................................... 122

Figure 7.29: 2D image of modules with 8 " x 8" x 0.03 " and 8" x 8" x 0.09 "

water-filled debonds.................................................. 122

Figure 7.30: 2D image of modules with 8" x 8" x 0.03 " and 8" x 8" x $0.09 "$ water-filled debonds with FIR.......................................... 122

Figure 7.31: 3D plot of the entire deck module with various water-filled defects..... 123

Figure 7.32: FIR 3D plot of the entire deck module with various water-filled defects

Figure 7.33: Signal analysis of the water-filled defects data. 124 
Figure 7.34: Signal analysis of the water-filled defects data with FIR 125

Figure 7.35: Layout of the defect for 1.5GHz ground-coupled antenna GPR test...... 126

Figure 7.36: Results of GPR test showing 12" x 12 " x 0.03 " and 8" x 8" x 0.06 " water-filled debonds.

Figure 7.37: Results of GPR test (FIR) showing 12" x 12" x 0.03 " and 8" x 8" x 0.06 "

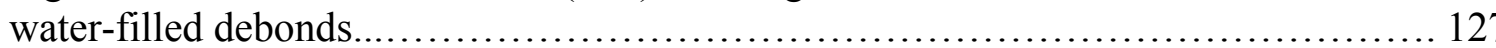

Figure 7.38: Results of GPR test showing 12" x 12" x 0.06 " water-filled debond..... 128

Figure 7.39: Results of GPR test (FIR) showing 12" x 12" x 0.06 " water-filled debond.

Figure 7.40: Results of GPR test showing 8" x 8" x 0.03 " and 8" x 8" x 0.09 " water-filled debond.

Figure 7.41: Results of GPR test (FIR) showing 8" x 8" x 0.03 " and 8 " x 8" x 0.09 " water-filled debond.

Figure 7.42: 3D map showing all the water-filled debonds...................... 130

Figure 7.43: Layout of the defects for $1.5 \mathrm{GHz}$ ground-coupled antenna GPR test...... 131

Figure 7.44: Results of GPR test showing smaller sized water-filled debonds......... 132

Figure 7.45: Results of GPR test (FIR) showing smaller sized water-filled debonds.... 132

Figure 7.46: 3D map showing all the water-filled debonds..................... 133

Figure 7.47(a): 2D GPR data collected using linear gain setting ................... 133

Figure 7.47(b): 2D GPR data (FIR) collected using linear gain setting................. 134

Figure 7.48: Pictures showing 4" thick low profile deck that was loaded until failure.. 136

Figure 7.49: 3D GPR profile of the 4" thick low profile failed deck............... 137

Figure 7.50: 2D GPR profile of the "failed region" in the 4" thick low profile failed deck.

Figure 7.51: 2D GPR profile of the "defect-free region" in the 4" thick low profile failed deck. 
Figure 7.52: Defect layout of delamination module.

Figure 7.53: 2D scan of delamination embedded within top flange of FRP bridge deck module....

Figure 7.54: 2D scan of defect free FRP bridge deck module. 140

Figure 7.55: 2D scan of delamination embedded within bottom flange of FRP bridge deck module.

Figure 7.56: 2D linear scan of delamination embedded within top flange of FRP bridge deck module.

Figure 7.57: 3D map of delamination module constructed by the constant gain data set.

Figure 7.58: (a) 512 points 2D scan (b) 1024 points 2D scan...................... 142

Figure 7.59: A constant gain 2D scan of 10ns range.......................... 143

Figure 7.60: Defect layout for blind test.................................... 145

Figure 7.61: FIR 2D scan of blind test revealing first two defects................. 146

Figure 7.62: FIR 2D scan of blind test revealing third defect.................... 146

Figure 7.63: FIR 2D scan of blind test revealing the fourth defect................. 146

Figure 7.64: FIR 2D scan of blind test revealing the fifth defect................... 146

Figure 7.65: FIR 2D scan of blind test revealing the sixth defect.................. 147

Figure 7.66: FIR 2D scan of blind test confirming the fifth defect shown

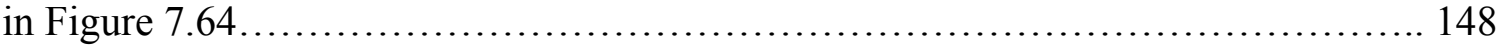

Figure 7.67: 3D map showing the location of the water-filled defects............... 148

Figure A1: RADAN main operating screen................................. 160

Figure A2: Click to apply FIR filter.......................................... 161

Figure A3: Input necessary information for FIR filter application................ 162

Figure A4: Start a new project for $3 \mathrm{D}$ construction........................... 162

Figure A5: Create a new RADAN bridge project............................. 163 
Figure A6: Adding necessary files for 3D construction........................... 164

Figure A7: Attach a dummy macro to all files................................... 164

Figure A8: Provide 3D file parameters ......................................... 165

Figure A9: Pick the beginning of each file ...................................... 166

Figure A10: Initiate the stitching of the files..................................... 166

Figure A11: 3D "stitched" file................................................ 167

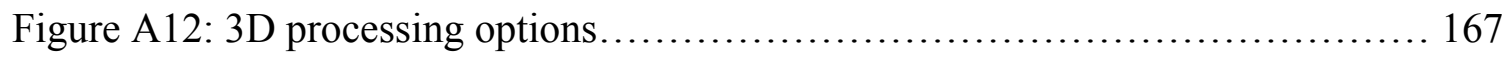




\section{LIST OF TABLES}

Table 3.1 Thermal properties of some materials.............................. 24

Table 6.1: The recorded data sheet for the specific heat value test................ 83

Table 6.2 Density table of round 2" diameter FRP and WS specimen................ 84

Table 6.3 Density table of square FRP specimen............................. 84

Table 6.4 Material properties of GFRP, wearing surface, and water................ 85 


\section{Chapter 1 \\ INTRODUCTION}

\subsection{BACKGROUND}

The advancement of Fiber Reinforced Polymer (FRP) technology has enabled the use of FRP bridge decks as possible replacement for many obsolete in-service concrete bridge decks. These composite decks are manufactured in factory through a pultrusion process. The FRP composites can be pultruded into various shapes and sizes which make it very attractive since it can cater to many different applications, such as bridge decks, support beams, and rebars, just to name a few.

While all of these composite components are manufactured under a control environment with high quality controls, subsurface defects such as cracks, voids, debonds, and delaminations could still be developed during the manufacturing process. Besides, subsurface defects could also develop during the installation phase, or during the service life of the structure due to various factors, such as weather, vehicle loads, wear and tear. The subsurface defects could adversely affect the integrity of the structure locally and globally. Coupled with the fact that FRP is still a relatively new technology with the in-service aging phenomena yet to be fully understood, it is imperative that an effective yet economical inspection procedure be employed to inspect and evaluate all inservice FRP structural components. At the same time, such inspection procedure could elevate the overall quality of the FRP products during the manufacturing (pultrusion) stage. 
This study investigates the use of Infrared Thermography and Ground Penetrating Radar as the two nondestructive evaluation (NDE) techniques for field inspection. Both techniques enable rapid data collection in the field environment and have a great potential for detecting subsurface defects.

The focus of this study is on the use of both Infrared Thermography (IRT) and Ground Penetrating Radar (GPR) for the detection of air- and water-filled subsurface debonds and delaminations in Fiber Reinforced Polymer (FRP) composite bridge decks. A debond refers to the discontinuity between the wearing surface and the underlying FRP deck while a delamination is a discontinuity within the flanges of the FRP deck.

The results of the previous research conducted at Constructed Facilities Center of West Virginia University have demonstrated the usefulness as well as limitations of the Infrared Thermography technique for subsurface defect detection in FRP bridge decks (Halabe et al. 2004a, 2004b, 2004c, 2004d, 2003a, 2002). While Infrared Thermography was found to be an excellent technique for detecting subsurface debonds between the wearing surface and the underlying FRP deck, its capability is very limited in terms of detecting delaminations within the flange of the FRP deck, specially for deck flanges overlaid with wearing surface. Also, Infrared Thermography was found to be sensitive to air-filled defects but the sensitivity reduced significantly for water-filled defects. On the other hand, GPR is more sensitive to water-filled defects. Therefore, it is important to combine the near-surface capability of Infrared Thermography with the deeper penetration capability of Ground Penetrating Radar (GPR) in order to arrive at a more robust hybrid system for detection of subsurface defects in FRP composites. However, research still needs to be conducted on GPR data analysis for FRP bridge decks and on 
developing a procedure to effectively combine the output of GPR and Infrared Thermography based systems.

While Infrared Thermography is more sensitive to near-surface defects (Halabe et al. 2004a, 2004b, 2004c), GPR uses electromagnetic waves to assess the condition at greater depths (Halabe et al. 1995). Also, unlike ultrasonics, GPR allows rapid data collection and does not require the use of a couplant between the antenna and the deck surface. GPR has been used in an air-launched mode by several researchers for concrete bridge deck and pavement applications and has great promise for field use (Maser et al., 2002a, 2002b). Some researchers have highly recommended the combination of Infrared Thermography and GPR as a more robust technique for concrete deck assessment (Maser et al. 2002a). Preliminary investigation by Jackson et al. (2000) also indicates that such a combination could be developed as a potentially effective tool for detection of debonds in FRP wrapped members.

\subsection{RESEARCH OBJECTIVES AND SCOPE}

The objective of this research is to investigate the use of GPR as well as Infrared Thermography for subsurface defect detection in FPR bridge deck specimens. Moreover, a finite element heat transfer model will be used to compare theoretical predictions with the actual results obtained from the IRT tests. The specific research objectives of this study are as follows:

- Investigate the use of both Infrared Thermography and GPR for nondestructive evaluation of FRP bridge decks using laboratory deck specimens. This investigation includes the inspection using GPR and IRT on the following: 
- deck specimens with debonds between the wearing surface and the FRP deck

- deck specimens with delaminations within the top flange of the FRP deck, with and without wearing surface on the top

- deck specimens with delaminations within the bottom flange of the FRP deck (GPR only)

- Compare the advantages and disadvantages of Ground Penetrating Radar with Infrared Thermography based on the experimental results

- Investigate the usefulness and effectiveness of the $1.5 \mathrm{GHz}$ ground-coupled antenna and the $2.0 \mathrm{GHz}$ horn antenna made by Geophysical Survey Systems, Inc. (GSSI). The antennas will be used to test both water and air-filled subsurface defects. The ability to locate the defects, the precision of the detection, and the information extracted from both antennas pertaining to the defects will be discussed.

- Develop a Finite Element (FEM) Heat Transfer Model for FRP bridge deck. The goal of the FE modeling is to study the effect of various defects thickness. The FEM results will be compared to the infrared images obtained in this study. The FEM model requires input parameters such as density, emissivity and thermal conductivity of the material. These parameters were measured in the laboratory for wearing surface and flange of low-profile FRP deck.

- Propose a Test Procedure for FRP Bridge Decks using GPR and IRT techniques. While GPR and IRT are proven nondestructive testing techniques, it is important to standardize the testing procedure as well as the test settings. Such standardization would help minimize many human errors while ensuring an 
effective inspection. A test procedure will be proposed at the end of this study for GPR and Infrared testing. This test procedure could be used in the future to further develop a Standard Field Testing Procedure.

\subsection{ORGANIZATION}

This dissertation consists of 8 chapters. Chapter 1 presents an introduction of nondestructive testing in general, as well as describes the objectives of this research. Chapter 2 includes the literature review of nondestructive testing using infrared thermography and ground penetrating radar. Chapter 3 discusses the underlying theories behind infrared thermography and ground penetrating radar. Chapter 4 describes the infrared and the ground penetrating radar equipment used in this study. The test specimens and the procedure for creating defects are also discussed in details. Chapter 5 discusses the infrared thermography results. It covers the results of using heater, heating blanket, and solar radiation as active heat sources. Chapter 6 presents the results from Finite Element heat transfer modeling. Description of the finite element model, the thermal properties obtained through experiments, and the results predicted using the model are discussed in this chapter. Chapter 7 is devoted to the experimental study using ground penetrating radar. The feasibility of using both ground- and air-coupled antennas as subsurface defect detection tool for FRP bridge deck is carefully discussed. Chapter 8 summarizes the research findings of this study. Future recommendations are also included in this chapter. This is followed by the Reference section and an appendix describing the post-processing steps used for GPR data analysis. 


\section{Chapter 2}

\section{LITERATURE REVIEW}

The history of ground penetrating radar (GPR) and infrared thermography (IRT) has been briefly reviewed in this chapter. The advancement of technology has brought incredible improvements for these nondestructive testing techniques. The applications as a result of such advancement has also been discussed in this chapter.

\subsection{HISTORY OF GPR AND IRT}

\subsubsection{GPR}

The theory behind electromagnetic waves and its reflections were first developed by James Clerk Maxwell in 1864. It was later confirmed by Heinrich Hertz in 1886 . In 1924, Sir Edward Victor Appleton utilized the basic electromagnetic reflection principles to estimate the height of the ionosphere, a layer in the upper atmosphere that reflects long radio waves. In 1935, British physicist Sir Robert Watson-Watt created the first practical radar system. Entering World War II, the British had constructed a network of radar systems along England's south and east coasts (Smemoe, 2000).

It is believed the first GPR survey was performed by German geophysicist W. Stern in 1929 at Austria (Olhoeft, web site). GPR did not gain prominence until the late 1950's when the radar systems in US Air Force planes penetrated through the ice in Greenland. It caused the pilot to misinterpret the altitude of the planes and crash into the ice. John C. Cook proposed to utilize radar for subsurface reflections detection in 1960 
(Cook, 1960). Cook and others continued to develop radar systems to detect reflections beneath the ground surface (Moffatt and Puskar, 1976).

The GPR system created by Moffatt and Puskar in 1976 used an improved antenna that gave a better target-to-clutter ratio and was able to detect subsurface reflections with great accuracy. Moffatt and Puskar (1976) were able to locate an underground tunnel, a fault, and mines. They concluded that GPR is a useful tool for detection of subsurface anomalies and exploration of subsurface soils variations. Moffatt and Puskar (1976) also presented some basic theory on GPR and equations for computing subsurface wave velocities.

\subsubsection{IRT}

Many ancient civilizations believed to have used their hands as a thermal imaging system, with the fingers acting as sensors and the brain interpreting the changes in temperature. The practice had enabled them to effectively evaluate, or even isolate, the changes of temperature at a specific area.

Hippocrates wrote in 400 B.C., "In whatever part of the body excess of heat or cold is felt, the disease is there to be discovered." The ancient Greeks immersed the body in wet mud. The area that dried more quickly indicated a warmer region, and was considered the diseased tissue (Hodge Jr., 1987).

The practice of sensing body temperature using hands continued well into the sixteenth and seventeenth centuries. Galileo made a thermoscope from a glass tube, which is believed to be the first thermometer. This device, however, did not have a scale since a conforming set of temperature scale was not established at the time. Fahrenheit 
then fixed a lower point by using salt with ice water and an upper point using boiling water at 212 degrees. Obviously, this set of scale is formally denoted as the Fahrenheit scale to honor Fahrenheit.

In 1742, Celsius created a decimal scale which focuses the zero degree as the boiling point of water and 100 as the freezing point. That scale was later reversed by a Swedish named Linnaeus. In 1868, Prof. Carl Wunderlich of Leipzig created the first set of temperature charts on individual patients with a wide range of diseases. He also proposed the creation of modern day clinical thermometer (Ring, 1997).

In 1877, Lehmann established that Cholesteric esters have the property of changing color with temperature. This discovery established the use of liquid crystal as another method of evaluating skin temperature. It is worth noting that the methods described before are both contact methods. The use of infrared thermal imaging did not gain popularity until the last 30 years.

The astronomer, Sir William Herschel, discovered the existence of infrared radiation when he tried to measure the heat of each of the rainbow spectrum cast on a table in a darkened room. He found the highest temperature to fall beyond the red end, which he reported to the Royal Society as Dark heat in 1800. His son, Sir John Herschel, managed to record the heat rays on the infra red side of red by creating an evaporograph image using carbon suspension in alcohol. That was believed to be the very first thermo image known to humans. By using the same principle, sophisticated thermal imaging equipment were later developed to accommodate military, industrial, and medical applications. 


\section{$2.2 \quad$ INFRARED THERMOGRAPHY}

In 2001, Miceli et al. used infrared imaging as the tool for monitoring the health of FRP structures with debonds and delaminations. They have found that surface anomalies due to staining and non-uniform wear causes complication in the data interpretation. They also concluded that the presence of moisture in the defects caused an inaccurate estimation of the defects.

In recent years, seismic retrofitting of bridge columns and rehabilitation of concrete structures using fiber reinforced polymer (FRP) wraps are becoming more and more popular. Traditionally, there are three main methods of wrapping. These are hand lay-up, pre-cured shells, and machine wraps. Though these additions to the existing infrastructures provide great improvements, each of these wrapping methods may end up creating voids or defects between the FRP sheet and the underlying structural component. Infrared thermography has proven to be an excellent method to detect such subsurface voids because of its remote inspection capability, its short inspection time, and its convenient means of data archival (Johnson et al., 1999).

Mtenga et al. (2001) found infrared thermography to be a very efficient method for quality assurance in FRP retrofitted structures. In their study, a double-tee (DT) concrete beam retrofitted with a CFRP wrap was nondestructively tested. They've used heat gun as their primary means of heat source. They have found IRT to be extremely useful in the void detection.

Brown and Hamilton III (2003) have conducted infrared thermography NDE tests on small-scale specimens created in the laboratory setting and on four full-scale AASHTO Type II concrete girders that were loaded to failure. The damaged girders were 
then strengthened by bonding FRP sheet on the bottom face of each girder. The laboratory tests concluded that IRT thermography is useful in evaluating the bond between FRP strengthening systems and concrete. They have also captured digital IRT images containing pixel-by-pixel temperature data. These digital data enabled an effective quantitative analysis of debonded areas. They also found that the effectiveness of IRT thermography in detecting debonds between the FRP and concrete decreases as the thickness of the FRP increases (Brown and Hamilton III, 2003).

\subsection{GROUND PENETRATING RADAR}

Barnes and Trottier (World Wide Web, 1999) surveyed seventy-two bridge decks using ground penetrating radar from 1996 to 1999. They found that ground penetrating radar can detect deterioration with a higher accuracy and less variability than the traditional method such as chain drag. This project further enhanced the accountability of ground penetrating radar and also helped reduce the cost of future maintenance with a more accurate procedure.

Ground penetrating radar has become an established technology for subsurface exploration purposes that involved geological application as well as many Civil Engineering applications. Applications such as groundwater investigations and road inspection using GPR with 2-D data acquisition are considered normal practice in the United States of America as well as many European countries. In recent years, however, many researchers have been focusing on developing 3-D data acquisition methods for alternative applications such as utility detection, landmine detection, just to name a few (Groenenboom and Yarovoy, 2000). Since subsurface targets such as utility lines, 
landmines, etc., may not necessarily be detected along a single line, broader plots of subsurface profile become necessary. This essentially leads to a need for threedimensional data acquisition using ground penetrating radar (Groenenboom et. al., 2001). The 3D GPR test has also greatly improved the traditional 2D GPR test as more information can be obtained in a single pass.

Aside from utilizing 3D GPR data acquisition for various applications, such as marine geology, many researchers are concentrating on developing imaging algorithms to physically explain and describe the datasets obtained through 3D GPR data acquisition. There are three important parameters that must be considered in the imaging algorithm in order to obtain a good image of the subsurface. The three parameters are the wave speed, the polarization, and the radiation characteristics of the source and the receiver antennas (Van der Kruk, 2001). Radzevicius and Daniels (2000) showed that when the polarization of the electric field is parallel to a pipe, a maximum reflection from that pipe would be obtained.

Although there exist several 3D imaging algorithms for GPR data based on scalar (seismic) imaging algorithms, many researchers turned their attention to incorporate the radiation patterns of source and receiver antennas and the characteristic of electromagnetic waves in the imaging algorithms for GPR data (Wang and Oristaglio, 2000). For instance, Van der Kruk et al. (2001) have compared the resolution functions of 3D multi-component imaging algorithms with 3D single-component imaging algorithms for ground penetrating radar data. They have found that the multi-component imaging algorithms were able to provide more information than that of single-component imaging algorithms. 


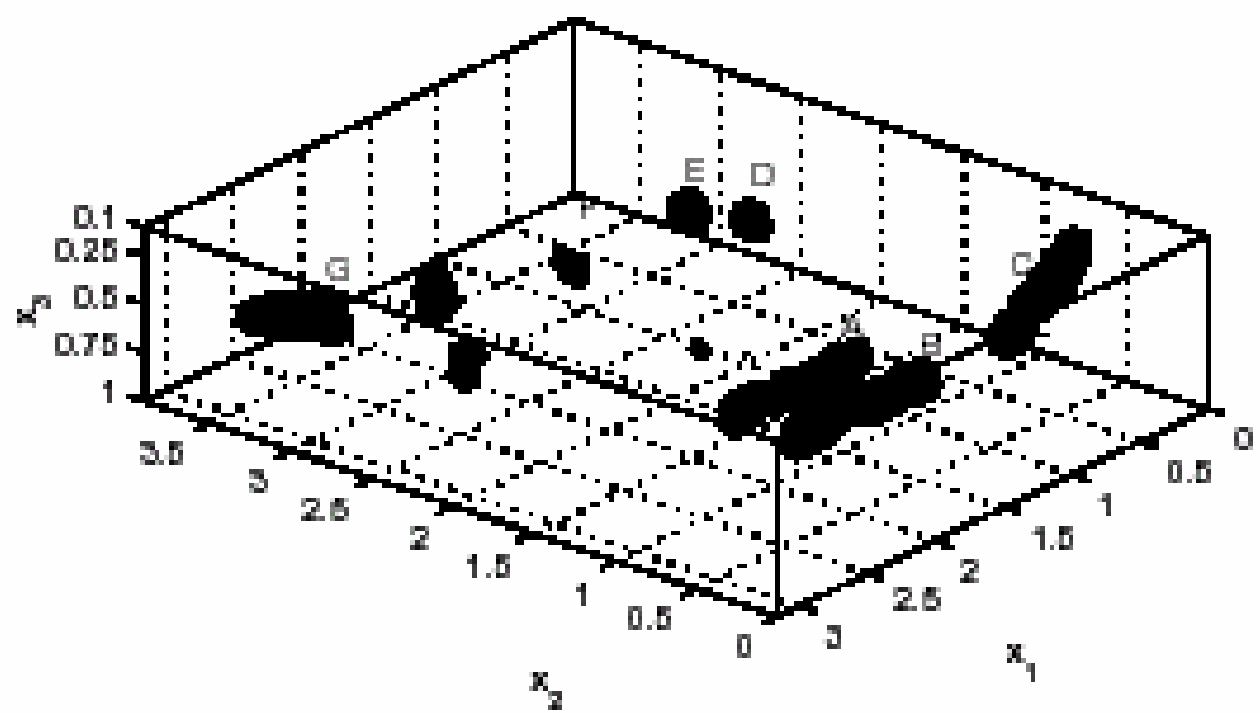

Figure 2.1 Multi-Component Imaging Results (Van der Kruk, 2001). 


\section{Chapter 3}

\section{BASIC THEORY}

\subsection{BASICS OF GROUND PENETRATING RADAR (GPR)}

Ground Penetrating Radar (GPR) operates by transmitting electromagnetic energy into the probed material and receiving the reflected pulses. The transmitted EM pulses are reflected as they encounter discontinuities. The discontinuity could be a boundary or interface between materials with different dielectrics or it could be a subsurface object such as debond or delamination. The antenna receives the pulses with varying amplitudes and arrival times. The amplitudes of the received echoes and the corresponding arrival times can then be used to determine the nature and location of the discontinuity. It is important to realize that the recorded arrival time is two-way travel time. The measurements have to be conducted carefully in order to get meaningful post-processing results. The reflected pulses are displayed on an oscilloscope as a time-series of pulses, known as waveform. These waveforms have been used to determine the depth of the asphalt layer, the thickness of the pavement, debonding of asphalt from concrete, and delamination of concrete (Halabe et al. 2003c, Carter et al. 1995). With the advancement in GPR technology, especially the increase in frequency of commercially available GPR antennas and better data processing software, GPR can now be used for subsurface condition assessment in materials consisting of thin layers such as Fiber Reinforced Polymer (FRP). Careful analysis of GPR waveforms can potentially help detect subsurface debonds between the wearing surface and the underlying FRP bridge deck, and delaminations within the flanges of the FRP deck. A major advantage of GPR over 
techniques such as Infrared Thermography is its ability to attain deeper penetration including evaluation of the bottom flange from the top surface of the deck itself, with no access required to the bottom side.

Velocity and attenuation of radar waves in the transmitted media directly affect the output waveform. Both the velocity and the attenuation of radar waves depend on the complex dielectric permittivity, which is related to the electromagnetic properties of solid particles, porosity, moisture content, and salt content of the medium (Halabe et al. 1993). Basically, most materials can be classified as either (a) conductors (e.g., metallic objects such as rebars in concrete deck), or (b) insulators, also known as dielectrics, such as asphalt, FRP, and concrete. Some media, such as water, are in between conductors and dielectrics depending on their purity (Halabe et al. 1993).

\subsubsection{Electromagnetic Properties and Radar Wave Propagation}

An FRP deck consists of multiple layers of different material with different dielectric properties. These layers include the wearing surface, FRP deck flanges and webs, debonds between the wearing surface and the FRP deck and possible delaminations within the deck flanges and/or web. The electromagnetic property of each of these layers is characterized by a property known as the dielectric permittivity. The relative dielectric permittivity $(\varepsilon)$ of a material is defined as the ratio of the actual dielectric permittivity of the medium $\left(\varepsilon_{\text {actual }}\right)$ to the permittivity of free space or vacuum $\left(\varepsilon_{0}\right)$.

$$
\varepsilon=\frac{\varepsilon_{\text {actual }}}{\varepsilon_{o}}
$$


The dielectric permittivity is a complex quantity and can be expressed as (Halabe et al. 1993):

$$
\varepsilon=\varepsilon^{\prime}+i \varepsilon^{\prime}
$$

where,

$$
\begin{aligned}
& \varepsilon=\text { relative complex dielectric permittivity of the medium } \\
& \varepsilon^{\prime}=\text { real part of relative dielectric permittivity } \\
& \varepsilon^{\prime \prime}=\text { imaginary part of relative dielectric permittivity }=\frac{\sigma}{\omega \varepsilon_{o}}
\end{aligned}
$$

where,

$$
\begin{aligned}
& \sigma=\text { dielectric conductivity of the medium }(\mathrm{mho} / \mathrm{m}) \\
& \omega=\text { angular frequency }(\text { radian } / \mathrm{sec})=2 \pi f \\
& \varepsilon_{o}=\text { dielectric permittivity of free space or vacuum }=8.854 \times 10^{-12} \text { farad } / \mathrm{m} \\
& f=\quad \text { frequency of the radar wave }(\mathrm{Hz})
\end{aligned}
$$

The real part of the relative dielectric permittivity $\left(\varepsilon^{\prime}\right)$ in Equation 3.2 is commonly known as the dielectric constant. The imaginary part ( $\left.\varepsilon^{\prime \prime}\right)$ is known as the loss factor. For one dimensional electromagnetic wave propagation along x-direction, the amplitude of the wave is given by Halabe (1990):

$$
A(x, t)=A_{o} e^{i(k x-\omega t)}
$$


where,

$$
\begin{aligned}
A_{o}= & \text { initial wave amplitude } \\
A(x, t)= & \text { wave amplitude at a distance ' } x \text { ' and time ' } t \text { ' } \\
k \quad & \text { complex wave number, which is related to } \varepsilon \text { as per the following } \\
& \text { relationship (Halabe 1990): } \\
& k=k_{R}+i k_{I}=\omega \sqrt{\mu_{o} \varepsilon_{o} \varepsilon}
\end{aligned}
$$

where,

$$
\begin{aligned}
& k_{R}=\quad \text { real part of complex wave number }\left(\mathrm{m}^{-1}\right) \\
& k_{I}=\text { imaginary part of complex wave number, also known as the } \\
& \text { material attenuation coefficient }\left(\mathrm{m}^{-1}\right) \\
& \mu_{o} \quad=\quad \text { magnetic permeability of free space }=4 \pi \times 10^{-7} \text { henry } / \mathrm{m}
\end{aligned}
$$

The real part of the wavenumber $\left(k_{R}\right)$ is related to the wavelength $(\lambda)$ of the propagating radar wave as per the following equation (Halabe 1990):

$$
k_{R}=\frac{2 \pi}{\lambda}
$$

For a medium with low conductivity, such as FRP decks, the attenuation coefficient, $k_{I}$, is given by (Halabe et al. 1993, Halabe 1990):

$$
k_{I}=\frac{377 \sigma}{2 \sqrt{\varepsilon^{\prime}}}
$$


The relationship between phase velocity $(V)$, wavelength $(\lambda)$, and frequency $(f)$ for electromagnetic waves is given as:

$$
V=\lambda f
$$

where,

$$
\lambda=\frac{2 \pi}{k_{R}}
$$

and,

$$
\omega=2 \pi f
$$

and hence,

$$
V=\frac{\omega}{k_{R}}
$$

For slightly conducting medium, the electromagnetic wave velocity is given by (Halabe et al. 1993, Halabe 1990):

$$
V=\frac{c}{\sqrt{\varepsilon^{\prime}}}
$$

where $c$ is the electromagnetic wave velocity in vacuum or air, which is the speed of light $\left(3 \times 10^{8} \mathrm{~m} / \mathrm{sec}\right)$.

The dielectric constant for the top layer can be computed from the reflection coefficient $R_{1,2}$. It is defined as the ratio of the reflected wave amplitude to the incident wave amplitude at an interface, and is given by (Halabe et al. 1993):

$$
R_{1,2}=\frac{\sqrt{\varepsilon_{1}{ }^{\prime}}-\sqrt{\varepsilon_{2}{ }^{\prime}}}{\sqrt{\varepsilon_{1}{ }^{\prime}}+\sqrt{\varepsilon_{2}{ }^{\prime}}}
$$

where the subscripts 1 and 2 denote the first and second media at the interface. 
The transmission coefficient, $T_{1,2}$ is defined as the ratio of the transmitted wave amplitude to the incident wave amplitude, and is given by (Halabe et al. 1993):

$$
T_{1,2}=1+R_{l, 2}=\frac{2 \sqrt{\varepsilon_{1}{ }^{\prime}}}{\sqrt{\varepsilon_{1}{ }^{\prime}}+\sqrt{\varepsilon_{2}{ }^{\prime}}}
$$

Reflection from a metal plate gives the amplitude of the incident radar wave (transmit pulse) because a metal plate is a perfect conductor, hence a perfect reflector. For a FRP bridge deck, the magnitude of the reflection coefficient $(R)$ for the top surface (air-FRP interface) can be written as (Halabe et al. 1995):

$$
R=-R_{1,2}=\frac{\sqrt{\varepsilon_{a}^{\prime}}-1}{\sqrt{\varepsilon_{a}^{\prime}}+1}=\frac{A}{A_{p}}
$$

where,

$A=$ amplitude of reflection from the top surface (i.e., air-FRP bridge deck interface)

$A_{p}=$ amplitude of reflection from the metal plate (negative of the incident amplitude) placed at the top of the FRP (in order to maintain a constant distance from the radar antenna)

$\varepsilon_{a}^{\prime}=$ dielectric constant of top surface of the FRP bridge deck

Hence,

$$
\varepsilon_{a}^{\prime}=\left[\frac{1+R}{1-R}\right]^{2}
$$


A $3 / 8 "(9.5 \mathrm{~mm})$ thick layer of wearing surface is usually placed on the top of the FRP deck modules in field construction to provide a riding surface and prevent slip of vehicles. For such decks, Equation (3.15) gives the dielectric constant of the wearing surface. If the wearing surface has not yet been placed, the GPR measurements of $A$ and $A_{p}$ would lead to dielectric constant of the FRP deck surface. It is important to note that the measurements of $A$ and $A_{p}$ are only possible with the use of an air-launched antenna and not with a ground-coupled antenna.

\subsubsection{Detection of Voids}

The detection of subsurface defects (voids, cracks, delaminations, etc.) is primarily a function of the changes in dielectric permittivity. Subsurface voids, debonds, or delaminations in FRP bridge decks may be filled with either air or water, which creates a discontinuity in the dielectric permittivity of the medium. Thus, if the change in dielectric permittivity is significant, then the gap (voids, debonds, or delaminations) appears to the radar as two reflectors close to each other (Halabe et al. 1995). If the entire wearing surface and/or the underlying deck have high moisture content, the attenuation of the radar wave also increases. This adversely affects the waveform amplitudes and makes it more difficult to analyze. Therefore, it is essential to conduct radar survey only during relatively dry weather when the overall FRP deck may have low moisture content. However, moisture inside the voids, debonds, or delaminations, are beneficial for radar detection since water-filled defects produce high amplitude reflections. Therefore, the optimal condition for conducting field GPR measurements is few days after it has rained, that is, after the overall deck had time to dry up but still has some moisture entrapped in the subsurface voids, debonds, or delaminations. 


\subsection{BASICS OF INFRARED THERMOGRAPHY (IRT)}

Heat transfer is defined as energy transfer due to temperature differences. Heat flows from the object at higher temperature to that at lower temperature. Because of the heat transfer, objects' temperature changes according to the heat flow. Hotter objects will become cooler while colder objects will become warmer. This heat transfer process will continue until a state of thermal equilibrium is achieved, meaning both objects attain the same temperature.

Temperature is a measurement of a material's thermal state but it is not a measurement of internal energy. Temperature can be measured in either relative or absolute terms. Relative temperatures are expressed in degrees "Celsius" $\left({ }^{\circ} \mathrm{C}\right)$ or "Fahrenheit" $\left({ }^{\circ} \mathrm{F}\right)$ for Foot-Pound-Second system (FPS) while absolute temperature is expressed in "Kelvin" (K) for the International System of Units (SI). The temperature units corresponding to the FPS system and SI system are related. The conversions between the two systems are as follows:

$$
\begin{aligned}
& T_{f}=\frac{9}{5} T_{c}+32 \\
& T_{k}=T_{c}+273.16
\end{aligned}
$$

where,

$$
\begin{aligned}
& T_{c}=\text { Temperature in degrees Celsius } \\
& T_{f}=\text { Temperature in degrees Fahrenheit } \\
& T_{k}=\text { Temperature in Kelvin }
\end{aligned}
$$

There are three modes of heat transfer between two objects, namely conduction, convection, and radiation. For simplicity purposes, the discussions of the three modes of 
heat transfer in this chapter deal with steady-state heat transfer. These modes of heat transfer occur on a molecular or subatomic scale.

\subsubsection{Conduction}

Conduction is the transfer of heat between two media through direct contact. By definition, conduction occurs when molecules travel a very short distance $(\sim 0.65 \mu \mathrm{m})$ before colliding with another molecule to initiate the energy exchange. The energy will be moved from higher temperature sites to lower temperature sites. Thermal conductivity is defined as the ratio of the heat flux to the temperature gradient and is measured in heat flow per unit area for a unit temperature difference across a unit thickness (Neville 1973). The equation relating the net heat flow through a material with its thermal conductivity is as follows:

$$
q_{\text {net }}=\frac{k}{d}\left(T_{h}-T_{l}\right)
$$

where,

$$
\begin{aligned}
& q_{n e t}=\text { net heat flow through a unit area of the deck per unit time }\left(\mathrm{W} / \mathrm{m}^{2}\right) \\
& k \quad=\text { thermal conductivity of the medium }\left(\mathrm{J} / \mathrm{m} / \mathrm{s} /{ }^{\circ} \mathrm{C} \text { or W/m} /{ }^{\circ} \mathrm{C}\right) \\
& T_{h} \quad=\text { temperature of the hotter side }\left({ }^{\circ} \mathrm{C}\right) \\
& T_{l} \quad=\text { temperature of the colder side }\left({ }^{\circ} \mathrm{C}\right) \\
& d \quad \quad=\text { thickness of the layer or the medium (m) }
\end{aligned}
$$

\subsubsection{Convection}

Convection is heat transfer by mass motion of a fluid such as air or water when the heated fluid is caused to move away from the source of heat, carrying thermal energy 
with it. Convection may arise from temperature differences either within the fluid or between the fluid and its boundary, other sources of density variations (such as variable salinity), or from the application of an external motive force. Convection is usually the dominant form of heat transfer in liquids and gases. There are two primary convection modes, namely the forced convection and the free convection. Forced convection happens when motion of the fluid is imposed externally (such as by a pump or fan). Free convection is convection in which motion of the fluid arises solely due to the temperature differences existing within the fluid. Naturally, the temperature at the object's surface, the ambient temperature, and the speed of the motion fluid (e.g. wind) are the factors that control the convective heat flow. The convective heat transfer between a deck surface and the surrounding fluid (air) can be obtained through Langmuir's equation, which is given as follows (Malloy 1969):

$$
q_{c}=1.947\left(T_{0}-T_{a}\right)^{5 / 4} \sqrt{\frac{(v+0.35)}{0.35}}
$$

where,

$$
\begin{array}{ll}
q_{c} & =\text { convective heat transfer from the surface }\left(\mathrm{W} / \mathrm{m}^{2}\right) \\
v & =\text { wind velocity }(\mathrm{m} / \mathrm{s}) \\
T_{o} & =\text { surface temperature of the } \operatorname{deck}\left({ }^{\circ} \mathrm{C}\right) \\
T_{a} & =\text { ambient air temperature }\left({ }^{\circ} \mathrm{C}\right)
\end{array}
$$




\subsubsection{Radiation}

Radiative heat transfer is the only form of heat transfer that can occur in the absence of any form of medium and as such is the only means of heat transfer through vacuum. Thermal radiation is a direct result of the movements of atoms and molecules in a material. Since these atoms and molecules are composed of charged particles such as protons and electrons, their movements result in the emission of electromagnetic radiation, which carries energy away from the surface. The behavior of such radiant emission can be described through Stefan-Boltzmann law, which states that the heat radiated by a body is directly proportional to the fourth power of its absolute temperature (Vanzetti 1972).

$$
q_{e}=\sigma \varepsilon T^{4}
$$

where,

$$
\begin{array}{ll}
q_{e} & =\text { total radiant emission from the radiating surface }\left(\mathrm{W} / \mathrm{m}^{2}\right) \\
\sigma & =\text { Stefan-Boltzmann constant }=5.673 \times 10^{-8} \mathrm{~W} / \mathrm{m}^{2} / \mathrm{K}^{4} \\
\varepsilon & =\text { emissivity value of the radiant object } \\
T & =\text { absolute temperature of the object }(\mathrm{K})
\end{array}
$$

While a body is radiating heat, its surface is also constantly receiving the radiation from the surroundings, resulting in the transfer of energy into the surface. Since the amount of emitted radiation increases with increasing temperature, a net transfer of energy from higher temperatures to lower temperatures results. Through the variation of the Stefan-Boltzmann law, the net radiation from any surface can be expressed as follows (Halabe and Maser 1986): 


$$
q_{r}=\sigma \varepsilon\left[\left(T_{o}+273\right)^{4}-\left(T_{a}+273\right)^{4}\right]
$$

where,

$$
\begin{array}{ll}
q_{r} & =\text { net radiation from the radiating surface }\left(\mathrm{W} / \mathrm{m}^{2}\right) \\
T_{a} & =\text { ambient air temperature }\left({ }^{\circ} \mathrm{C}\right) \\
T_{o} & =\text { temperature of the radiating surface }\left({ }^{\circ} \mathrm{C}\right)
\end{array}
$$

As per the Stefan-Boltzmann equation, the radiation heat transfer is directly

\begin{tabular}{|c|c|c|c|c|c|}
\hline Material & $\begin{array}{l}\text { Specific heat } \\
\left(\mathrm{J} \mathrm{kg}^{-1 \circ} \mathrm{C}\right)^{-1}\end{array}$ & $\begin{array}{l}\text { Density } \\
\left(\mathrm{kg} \mathrm{m}^{-3}\right) \\
\end{array}$ & $\begin{array}{l}\text { Heat capacity } \\
\left(\mathrm{J} \mathrm{cm}^{-3}{ }^{\circ} \mathrm{C}^{-1}\right) \\
\end{array}$ & $\begin{array}{l}\text { Thermal conductivity } \\
\qquad\left(\mathrm{W} \mathrm{m}^{-1}{ }^{\circ} \mathrm{C}^{-1}\right)\end{array}$ & $\begin{array}{c}\text { Thermal diffusivity }{ }^{\mathrm{a}} \\
\delta \times 10^{-6}\left(\mathrm{~m}^{2} \mathrm{~s}^{-1}\right) \\
\end{array}$ \\
\hline Air (as defect) & 700 & 1.2 & $0.8 \times 10^{-3}$ & 0.024 & 33 \\
\hline Aluminium & 880 & 2700 & 2.4 & 230 & 95 \\
\hline Brass $(65 \% \mathrm{Cu}, 35 \% \mathrm{Zn})$ & 380 & 8400 & 3.2 & 130 & 32 \\
\hline $\mathrm{CFRPb}(\perp$ fibers $)$ & 1200 & 1600 & 1.9 & 0.8 & 0.42 \\
\hline $\operatorname{CFRP}^{\mathrm{b}}(\overline{\|}$ fibers $)$ & 1200 & 1600 & 1.9 & 7 & 3.7 \\
\hline Concrete & 800 & 2400 & 1.9 & 1 & 0.53 \\
\hline Copper & 380 & 8900 & 3.4 & 380 & 110 \\
\hline Epoxy resin & 1700 & 1300 & 2.2 & 0.2 & 0.09 \\
\hline Glass & 670 & 2600 & 1.7 & 0.7 & 0.41 \\
\hline $\operatorname{GFRP}^{\mathrm{c}}(\perp$ fibers $)$ & 1200 & 1900 & 2.3 & 0.3 & 0.13 \\
\hline $\operatorname{GFRP}^{c}$ ( $\|$ fibers $)$ & 1200 & 1900 & 2.3 & 0.38 & 0.17 \\
\hline Lead & 130 & 11300 & 1.5 & 35 & 23 \\
\hline Nickel & 440 & 8900 & 3.9 & 91 & 23 \\
\hline Plexiglass $^{\mathrm{TM}}$ & - & 1200 & - & 0.2 & 0.25 \\
\hline Porcelain & 1100 & 2300 & 2.5 & 1.1 & 0.43 \\
\hline Steel (mild) & 440 & 7900 & 3.5 & 46 & 13 \\
\hline Steel (stainless) & 440 & 7900 & 3.5 & 25 & 7.1 \\
\hline Teflon $^{\mathrm{TM}}$ & - & - & - & 0.42 & 1.59 \\
\hline Titanium & 470 & 4500 & 2.1 & 16 & 7.6 \\
\hline Uranium & 120 & 17800 & 2.2 & 27 & 12 \\
\hline Water & 4180 & 1000 & 4.2 & 0.6 & 0.14 \\
\hline Zircaloy 2 & 280 & 6600 & 1.8 & 13 & 11 \\
\hline
\end{tabular}
proportional to the emissivity value, $\varepsilon$, which is therefore an important material property.

Table 3.1 lists the various thermal properties of some commonly used materials.

Table 3.1 Thermal properties of some materials (Maldague 1993)

(Vavilov 1980, p 182; Reynolds and Wells 1984, p 43; Tretout 1987, p 49; Touloukian and DeWitt 1970) ${ }^{a}$ Defined as $\delta=\mathrm{K} / \rho \mathrm{C}$, where $\mathrm{K}$ is the thermal conductivity, $\rho$ is mass density and $\mathrm{C}$ is the specific heat.

${ }^{\mathrm{b}}$ Carbon fiber reinforced polymer.

${ }^{\mathrm{c}}$ Glass fiber reinforced polymer. 


\subsubsection{Infrared Measurement}

At a temperature above absolute zero $\left(-273.16{ }^{\circ} \mathrm{C}\right)$, all objects radiate electromagnetic waves. The intensity of the radiation is directly proportional to the object's temperature and its emissivity value. Emissivity value is defined as the ratio of radiation emitted by an object to that from a blackbody source, which theoretically refers to the object that emits maximum amount of radiation at any given temperature.

Radiative heat transfer occurs in the infrared portion of the electromagnetic spectrum $(0.75 \mu \mathrm{m}$ to around $100 \mu \mathrm{m})$. Figure 3.1 shows the electromagnetic spectrum.

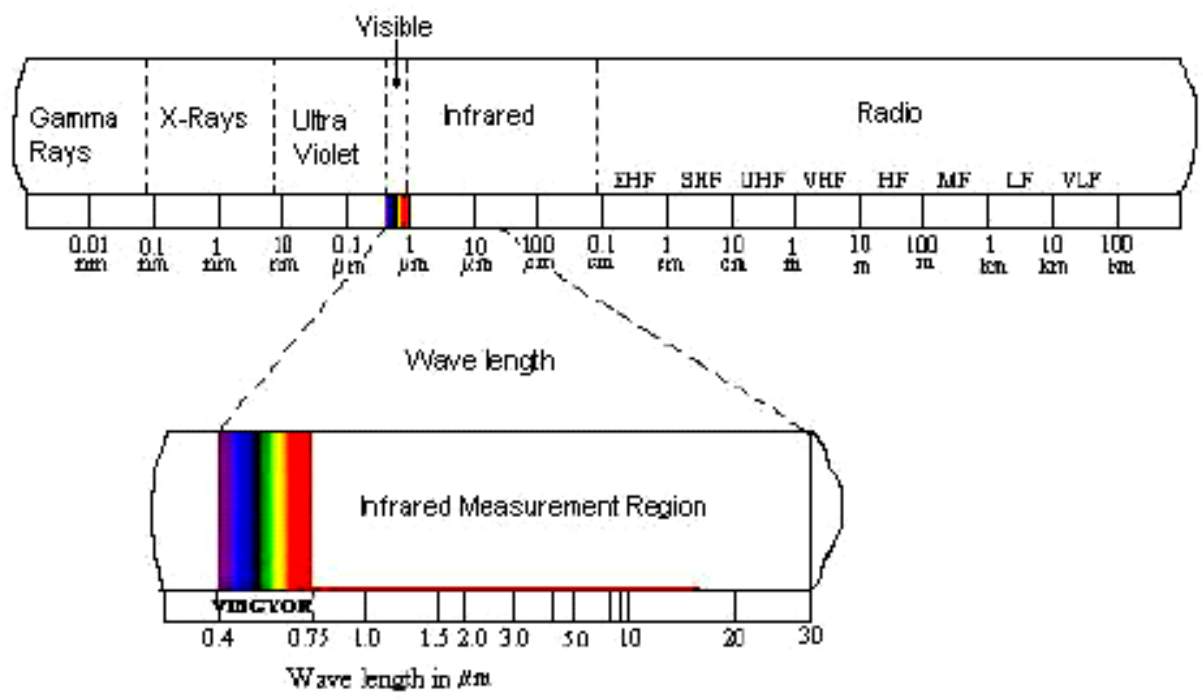

Figure 3.1 Electromagnetic spectrum showing the infrared measurement region (Kaplan 1999).

For practical purposes, most infrared measurements are conducted around $20 \mu \mathrm{m}$ (Kaplan 1999). The infrared measurement is made through infrared detectors that can sense the infrared radiant energy. Such devices translate the radiant energy into useful electrical signals proportional to the temperature of the probed objects. With the advancement of technology in recent years, the infrared thermal imagers are capable of 
producing thermal maps (thermograms) with various color palettes, including black and white scale.

Thermal imaging is conducted through two different approaches: the active thermography and the passive thermography. In active thermography, heat flow is produced with external heating or cooling of the structure. Because of this thermal excitation, the object with subsurface anomalies will display non-uniform surface temperatures. Conversely, the passive thermography relies solely on the existing temperature differences within the object. The passive scheme is commonly used to assess the state of industrial processes or during the manufacturing stage of certain products (Maldague and Moore 2001). 


\section{Chapter 4}

\section{EQUIPMENT AND TEST SPECIMENS}

\subsection{GPR EQUIPMENT}

The equipment used in the Ground Penetrating Radar portion of this study was manufactured by Geophysical Survey Systems, Inc. The SIR-20 data acquisition system was chosen based on the fact that it has two different channels that could accommodate various antennas with different frequencies. The SIR-20 system is also capable of integrating data collection and post-processing for instant results. Furthermore, the SIR20 system has the capability to take input from a geographic information system (GIS). The associated data processing software, RADAN, is capable of 3D modeling, which drastically improves the post-processing process.

The SIR-20 system comes with an industrial strength notebook computer made by Panasonic and a mainframe, which serve as a bridge between the notebook computer and the antennas. Two types of antenna were chosen for this study.

The first type is a $2.0 \mathrm{GHz}$ air-coupled antenna, also commonly known as horn antenna. The horn antenna is optimized for non-contact high-speed scanning, and is the antenna with the highest frequency commercially available at this time. Typically, a horn antenna is mounted to the back of a vehicle for field testing. Since the current research is a laboratory study, a push-cart was modified to mount the horn antenna (Figure 4.1a). Such configuration will allow the survey of a small bridge deck within laboratory setting.

The second antenna is a $1.5 \mathrm{GHz}$ ground-coupled antenna (Figure 4.1b). While the horn antenna is optimized for high speed scanning, the ground-coupled antenna is 
well known for the high resolution radar images it produces because it is capable of transmitting more energy into the material. The $1.5 \mathrm{GHz}$ frequency is the highest commercially available frequency at this time for a ground-coupled antenna. The depth of viewing window for the $1.5 \mathrm{GHz}$ antenna is approximately 18 inches in concrete.

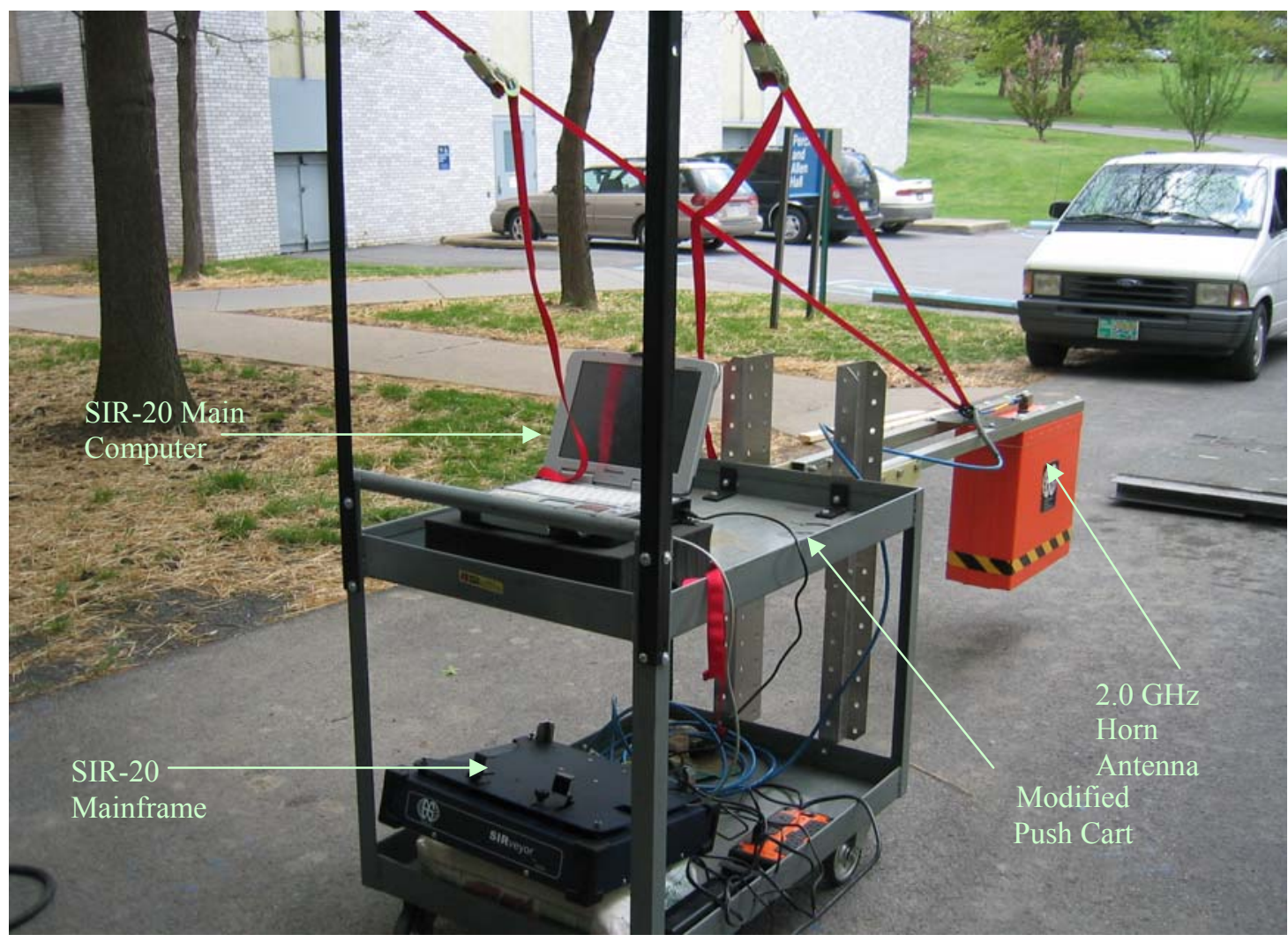

Figure 4.1(a): GPR system with $2.0 \mathrm{GHz}$ horn antenna on the modified push-cart.

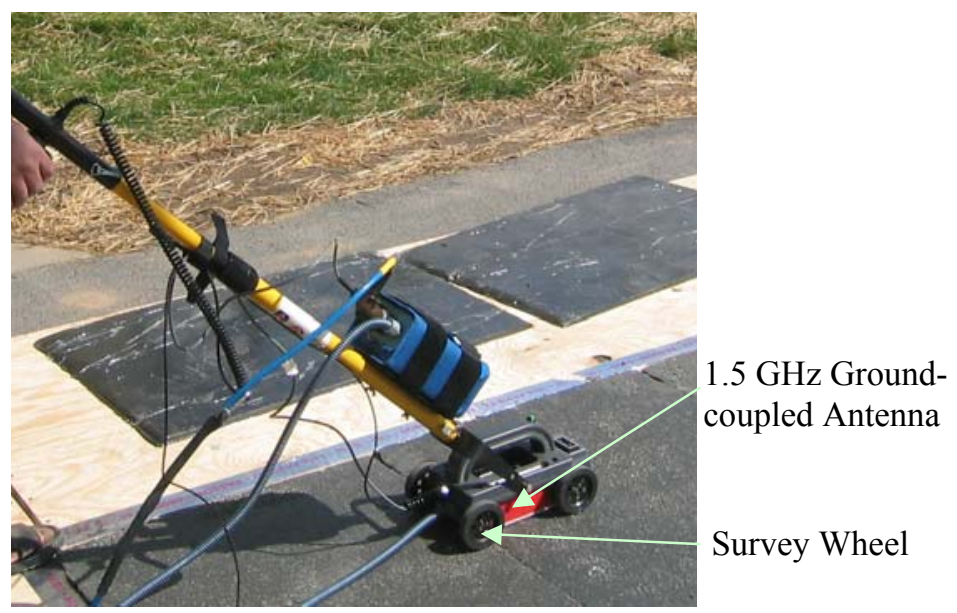

Figure 4.1(b): Hand-held 1.5 GHz ground-coupled antenna and survey wheel configuration. 
Figure 4.2(a) below shows the footprint of the $2.0 \mathrm{GHz}$ air-coupled antenna. The footprint was obtained by moving a steel rebar underneath the antenna. When the rebar reflects the electromagnetic energy sent by the antenna, the waveform of the scan will change from its original state. Such changes would determine the outer most boundary for the electric field generated by the antenna. The dimensions of this boundary, known as the antenna's footprint, is directly proportion to the height of the antenna from the ground because of the beam spread as shown in Figure 4.2(a). Therefore, it is important to calculate the beam spread angle, which is shown in the figure below.

\section{$\underline{\text { GPR - Horn Antenna Dimensions }}$}

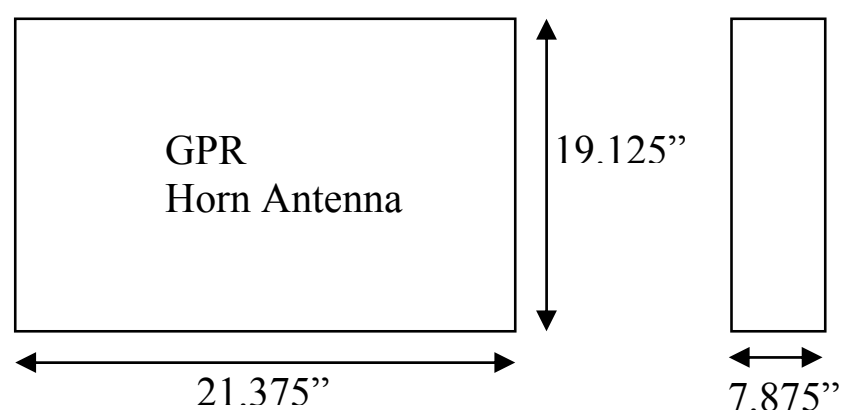

Air-coupled horn antenna's effective footprint and angle:

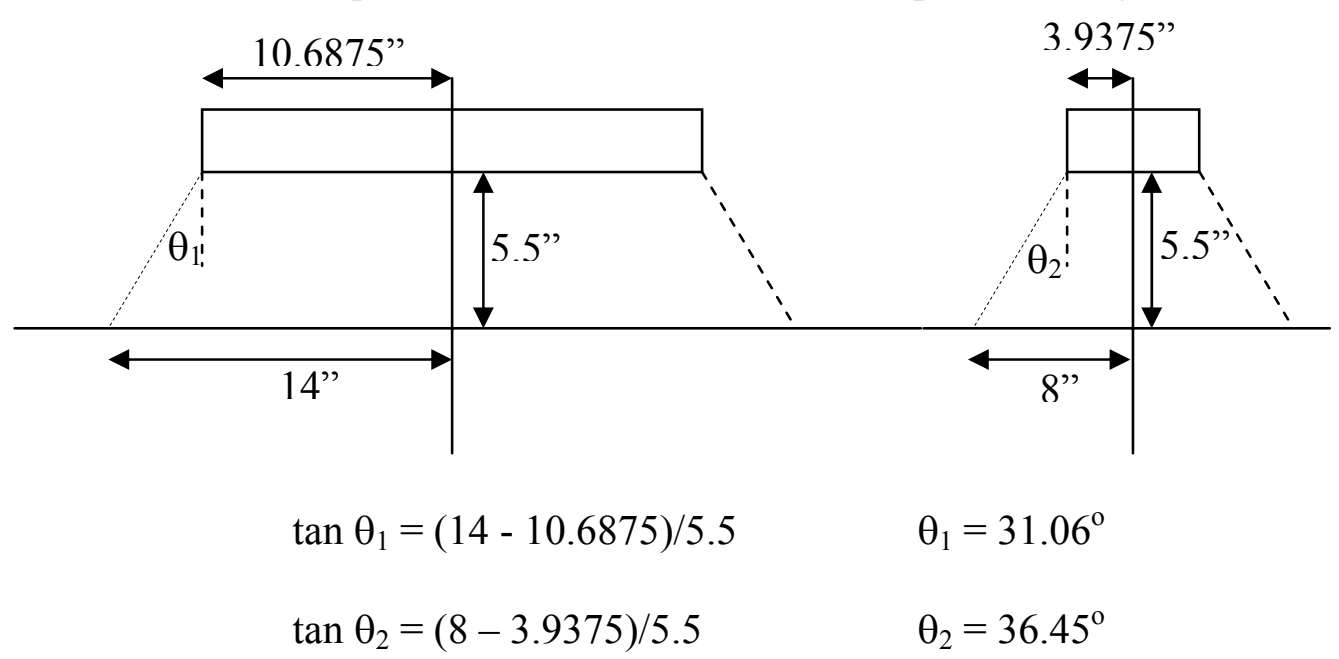

Figure 4.2(a): Footprint of the $2.0 \mathrm{GHz}$ air-coupled horn antenna. 
Figure 4.2 (b) shows the footprint of the $1.5 \mathrm{GHz}$ ground-coupled antenna. The footprint was obtained by moving a thin aluminum plate underneath the antenna. When the aluminum plate reflects the electromagnetic energy sent by the antenna, the waveform of the scan will change from its original state. Such changes would determine the outer most boundary (antenna's footprint) for the electric field generated by the antenna. This footprint is longer that the physical size (7" x 4") of the ground-coupled antenna, but narrower with a total of 2" effective footprint perpendicular to the line of survey.

\section{Ground-coupled antenna's footprint}
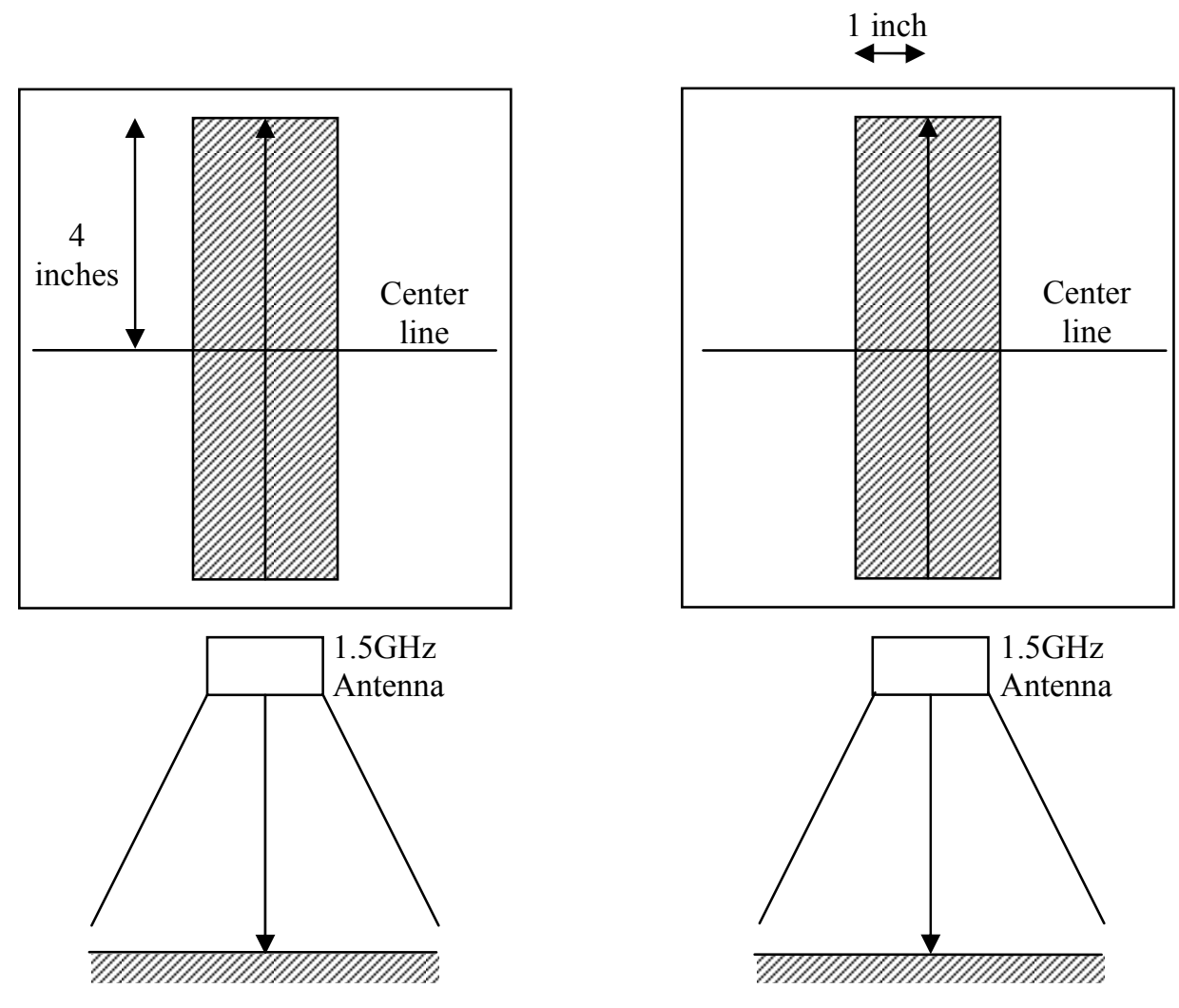

Footprint along the line of survey

Footprint perpendicular to the line of survey

Figure 4.2(b): Footprint of the $1.5 \mathrm{GHz}$ ground-coupled antenna. 


\subsection{INFRARED EQUIPMENT}

The ThermaCAM ${ }^{\mathrm{TM}}$ S60 (FLIR Systems) infrared monitoring system consists of an advanced digital infrared camera and associated image processing software. The ThermaCAM ${ }^{\mathrm{TM}}$ camera (shown in Figure 4.3) is a handheld, lightweight and a truly portable camera with a built-in $24^{\circ}$ lens. It also contains an integral digital color camera, a laser pointer, a 4" color LCD on a removable remote control, and a range of accessories. This camera can detect radiation in the spectral range of 7.5 to $13 \mu \mathrm{m}$.

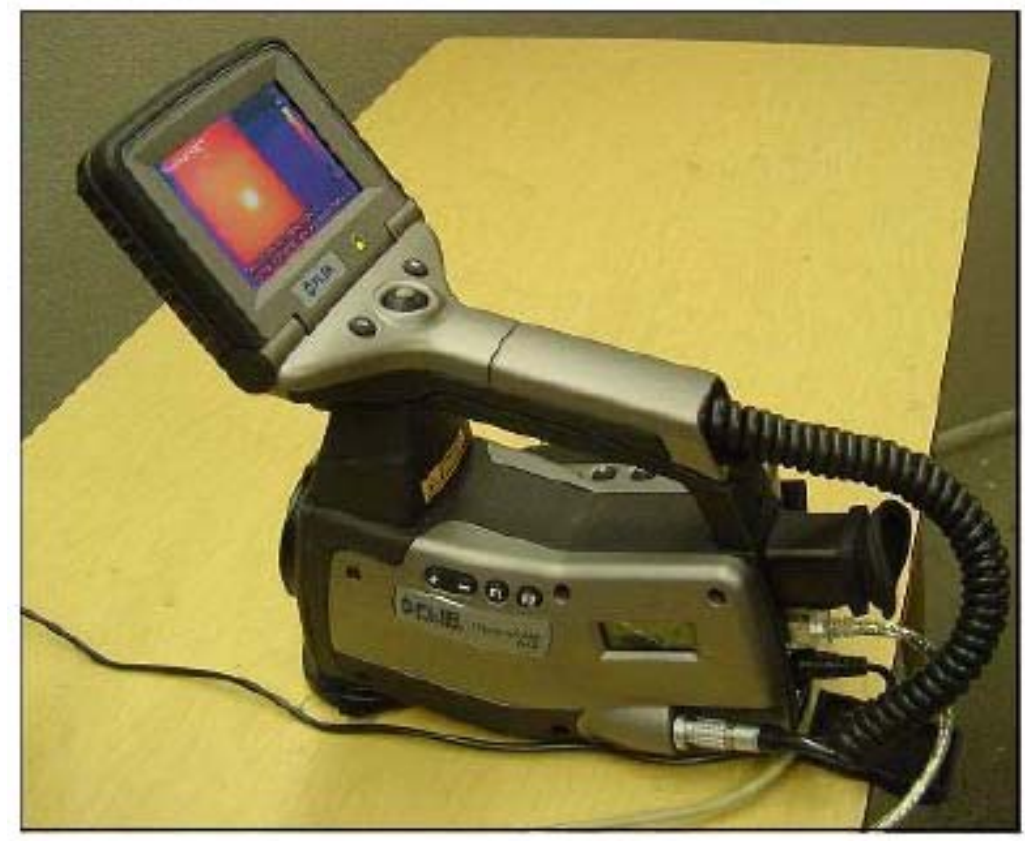

Figure 4.3 ThermaCAM ${ }^{\text {TM }}$ S60 infrared camera from FLIR Systems.

The type of detector in the camera is a focal plane array (FPA), uncooled microbolometer. The different temperature ranges available for the measurement are 0 to $+500^{\circ} \mathrm{C}\left(+32\right.$ to $\left.+932{ }^{\circ} \mathrm{F}\right),-40$ to $+120^{\circ} \mathrm{C}\left(-40\right.$ to $\left.+248{ }^{\circ} \mathrm{F}\right)$ and +350 to $1500{ }^{\circ} \mathrm{C}(+662$ to $+2732^{\circ} \mathrm{F}$ ). The measurements can be detected up to an accuracy of $\pm 0.06^{\circ} \mathrm{C}$ or $\pm 2 \%$ of the temperature reading. The imaging performance for the camera has a spatial resolution 
of $1.3 \mathrm{mrad}$ and can record images at a capture rate of up to 60 frames per second, noninterlaced. It is possible to capture and store images on a removable flash card or directly into a laptop computer which also houses the display software. The camera also features burst recording functionality that allows the user to record sequences of events into the internal RAM memory. Voice and/or text comments could be stored. The built-in digital color camera captures critical details, making reporting and analysis easy. The images can be analyzed either in the field by using the real-time measurement markers built into the camera software, or in a PC using FLIR Systems software (FLIR Systems 2002a).

The software that is used along with the camera is called as the ThermaCAM ${ }^{\mathrm{TM}}$ Researcher 2002. It deals with the live IRT images arriving through the camera interface and can also receive IRT images from other media, such as PC card hard disk from the camera. The software can be used to display the IRT images, record them on the disk, or analyze them later during the replay. The measurements can be made with the analysis tools like isotherm, spot meter, area and line. The images can be processed further to enhance their contrast. Since the camera captures fully radiometric digital images, a reference image can be subtracted from the full image sequence to achieve better results in terms of detectability of defects and to conduct a quantitative analysis (FLIR Systems 2002b).

\subsubsection{Heating Sources}

One of the objectives of this study is to advance the nondestructive testing technology using Infrared Thermography. In order to attain the goal, it is vital to explore both passive thermography and active thermography. 


\section{Solar Radiation}

The sun has long been considered a massive energy source for the earth. On a bright sunny day, solar radiation is often regarded as the perfect uniform heating source provided the wind is not interfering with the process. As Maser et al. (1990) pointed out, the sun produced excellent temperature differentials in concrete decks using IRT between 10 AM to 2 PM.

As good as it may be, though, solar radiation does indeed have weaknesses when dealing with IRT nondestructive testing. First of all, the availability of solar heating is heavily dependent on the unpredictable weather. In order to conduct a successful solar heating IRT test, the sky has to be clear most of the time. Also, as indicated in the previous chapter, wind velocity is one of the parameters that affects the overall heat transfer in convective mode. Therefore, it is important to have a relatively windless day to ensure uniform heating. It should be noted that solar radiation is a gradually increasing heat source (from morning to mid-day) which may not cause a sufficiently large thermal perturbation over a small period that is often needed for successful IRT testing.

Nonetheless, the study of solar heating on FRP bridge decks is crucial in further understanding and advancing the nondestructive testing using IRT.

\section{Electrical/Gas Heater}

To induce heat into the FRP bridge deck specimens thus creating an active heating, both electrical and gas heaters can be used. Generally, the commercially available heaters (both electrical and gas powered heaters) are capable of inducing a high level of heat into any object over a short period of time, thus resulting in high thermal 
stimulation. There are a wide range of heaters with various heating power available for selection. This provides an incredible amount flexibility to achieve a good active heating on FRP bridge deck, which will enable field testing even during cold days.

\section{Heating Blanket}

An electric heating blanket of plan size 36" x 36" (914mm x 914mm) and 1" (25mm) thickness (Figure 4.4) was purchased to apply uniform heating in this study. This heating blanket is rated at $1016 \mathrm{~W}$. The blanket is connected to an external temperature control box (Figure 4.4). The blanket has a double stitched inner liner of 3259 silicone fabric with an outer cover made from a tough vinyl fabric. The insulation of the blanket is an one inch thick Armaflex foam. The heating elemts are 2" wide knitted heating tape strips bonded to the inner liner. These multi-stranded (coils) heating elements are connected in a series configuration. The blanket also has a Type J thermocouple with a male plug for connection to an external temperature controller.

Although maximum allowable temperature of $163^{\circ} \mathrm{C}\left(325^{\circ} \mathrm{F}\right)$ can be produced by the blanket, the safe operational temperature range was limited to $32-60{ }^{\circ} \mathrm{C}(90-140 \stackrel{\circ}{\mathrm{F}})$ since higher temperatures over prolonged periods could damage the heating coils. To ensure that there is no overheating of the blanket before, during, and after the experiment, the external temperature control box with thermostatic control was used to cut off the power supply to the blanket once the desirable temperature is achieved.

One of the most intriguing features of the heating blanket is its ability to wrap around objects such as beams and columns. Since the heat is produced through electrical heating of coils, the heating blanket can be used in either vertical or horizontal position. 
As attractive as it may sound, the heating blanket does take a longer time to induce same amount of heat compared to the electrical or gas heaters in general. Also, since the heating blanket was constructed by connecting the heating elements in series, it is important to handle the heating blanket with care to ensure that none of the heating elements are damaged. A damaged heating element will cause the entire heating blanket (or a part of it) to cease functioning.

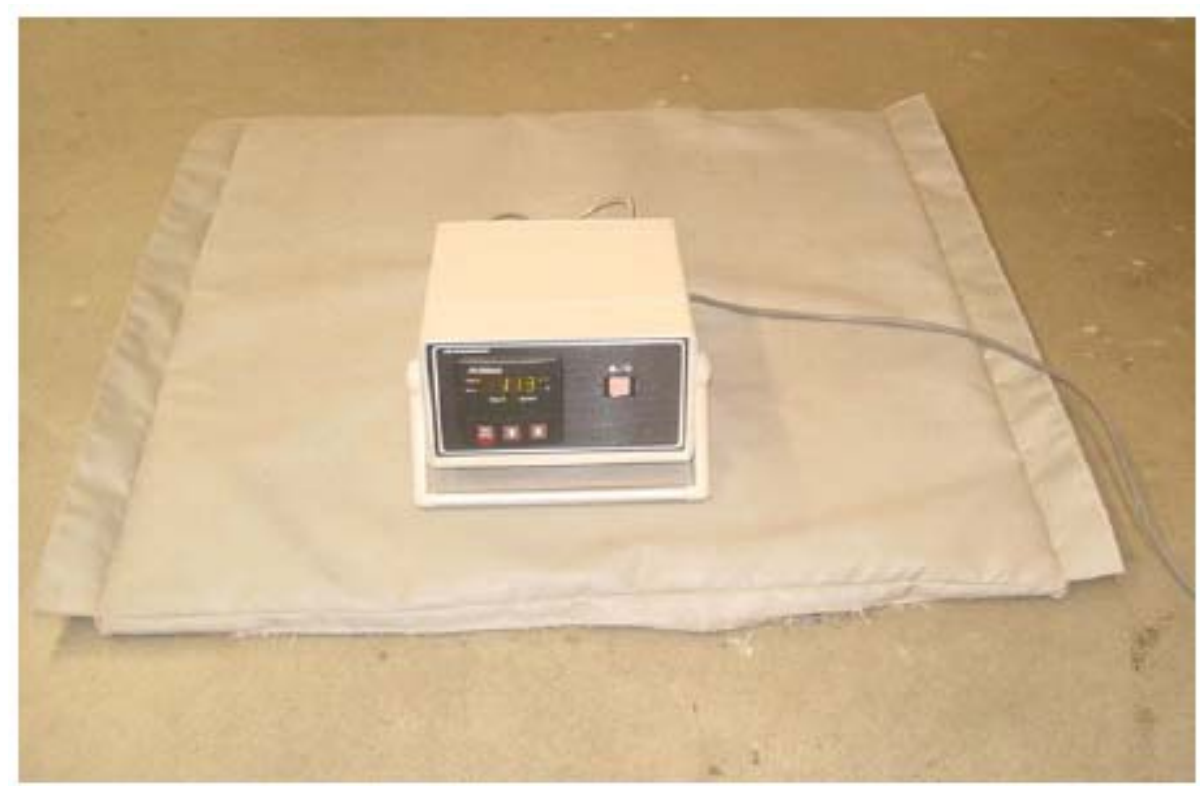

Figure 4.4: Heating blanket with external temperature control box.

\subsection{TEST SPECIMENS}

The multi-cellular shaped low-profile FRP bridge deck is made of E-glass fiber and vinyl ester resin. Figure 4.5 shows the cross section of the multi-cellular shaped FRP deck component of 30" (762 mm) width and 4" $(101.6 \mathrm{~mm})$ thickness. This bridge deck is designed to withstand AASHTO's HS20 loads in spite of the low deck thickness of 4" (101.6 mm). This is achieved by creating more fiber continuity between the web and the 
flange as well as by reducing the weight. This low-profile deck is cost effective and is manufactured with higher structural strength and lower weight than its predecessor.

The flanges and webs of the low-profile FRP bridge deck component were made of triaxial fabrics, continuous rovings and mats. The fibers continue from the flange to web and back to the flange. The resin used in this deck was vinyl ester resin which is a high elongation resin. This low-profile deck weighs about $10 \mathrm{lb} / \mathrm{ft}^{2}$ and has fiber volume fraction of approximately $50 \%$.

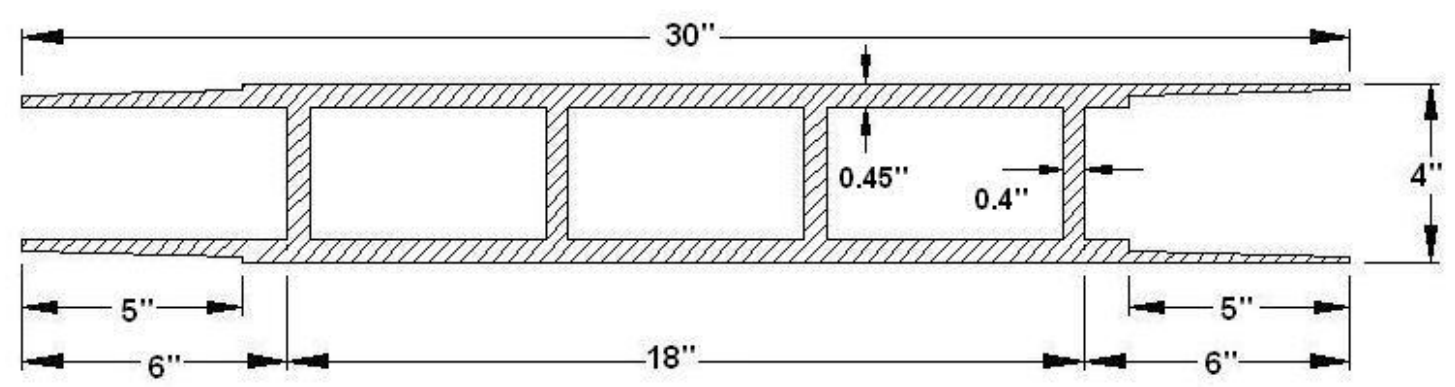

Figure 4.5: Cross-section of low-profile FRP bridge deck.

The ability to manufacture bridge deck in a factory setting thus ensuring good quality control is one of the most intriguing aspects of FRP bridge deck. Generally, the FRP products are first designed, and then manufactured through a pultrusion process. Pultrusion is a process that involves direct conversion of continuous fibers and resins into finished product of any size and shape, so long as a mold can be made. The first step to be considered in pultrusion process is the design and manufacturing of pultrusion die to strict tolerances of thickness, angularity and radius. The performing guides position the roving and mat dispensed from the creel and mat racks in the specified location, in the cross section of the product. Then the fiber reinforcements are made to pass through the wet-out bath and the injection chamber, where they are wetted by the thermoset resin, 
supplied under pressure. The extreme pressure forces out any air or excess resin from the fiber reinforcement as it enters the die. The part is subjected to multiple curing temperatures inside the die. The layers of reinforcements are mechanically fixed to each other, which results in a solidified laminate. The finished product exiting the die is then pulled by reciprocating pullers at a constant speed. The product is cut to the specified length with the aid of a moving cutoff saw. Figure 4.6 shows the schematic diagram of a basic pultrusion process.

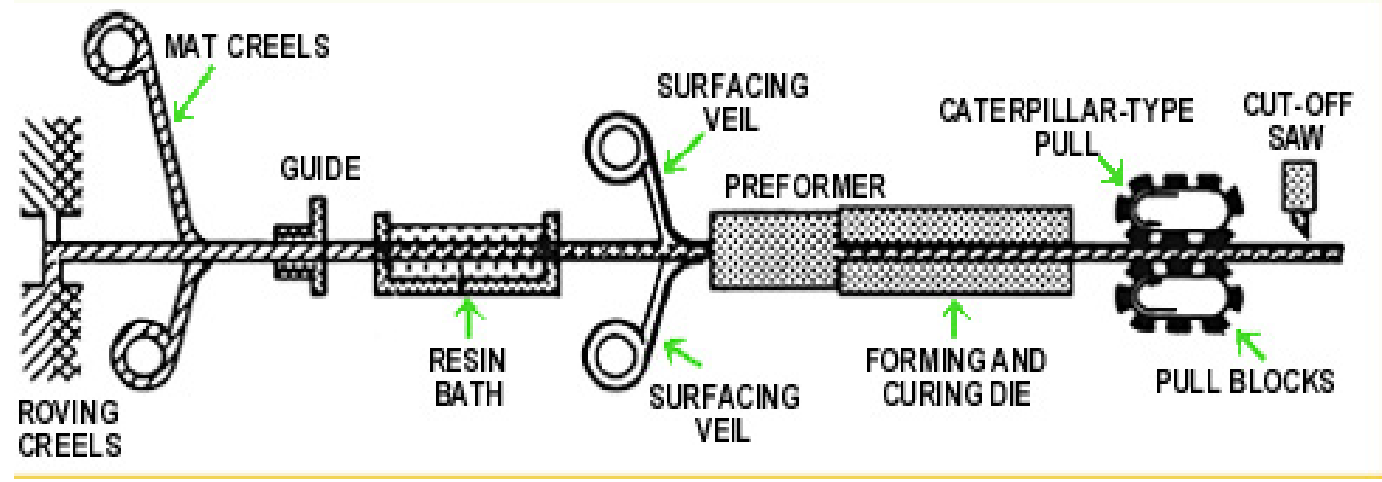

Figure 4.6 Schematic diagram of Pultrusion process.

The low-profile FRP bridge deck specimens used in this study were manufactured by Bedford Reinforced Plastics, Inc (BRP). Figure 4.7 shows the photographs taken at BRP during pultrusion of the low-profile FRP deck. 

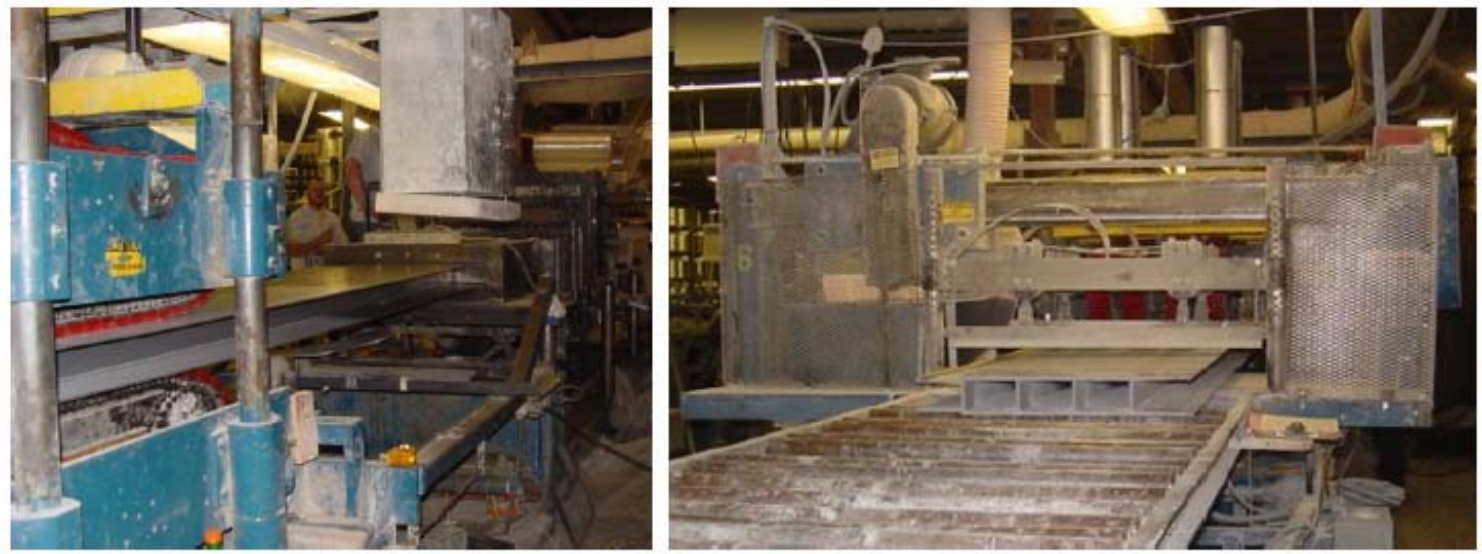

Figure 4.7: Photographs showing manufacturing of FRP bridge deck in a factory using Pultrusion process.

This study evaluates the applicability of both ground penetrating radar and digital infrared thermography systems to detect subsurface anomalies such as water-filled and air-filled debonds and delaminations. Debonds refer to discontinuities between the wearing surface and the underlying deck, while delaminations refer to discontinuities within the flanges of the deck itself.

\subsection{PREPARATION OF DEFECTS}

Debonds refer to the subsurface defects that are present at the interface between the wearing surface layer and the underlying FRP deck. In order to produce defects that could be used over and over again, modular wearing surfaces of dimensions 36 " x 24 " $\mathrm{x}$ $3 / 8 "(914.4 \mathrm{~mm} \times 609.6 \mathrm{~mm} \times 9.525 \mathrm{~mm})$ (Figure 4.8) were cast. The wearing surface module was made by mixing a gallon of Transpo Industries' Transpo T-48A Epoxy Resin, half a gallon of Transpo T-48B Hardener, and a 52 pounds bag of Transpo T-48 Powder component and cast over a light steel frame of dimensions 36 " x 24 " x $3 / 8$ " (914.4mm x 609.6mm x 9.525mm). 


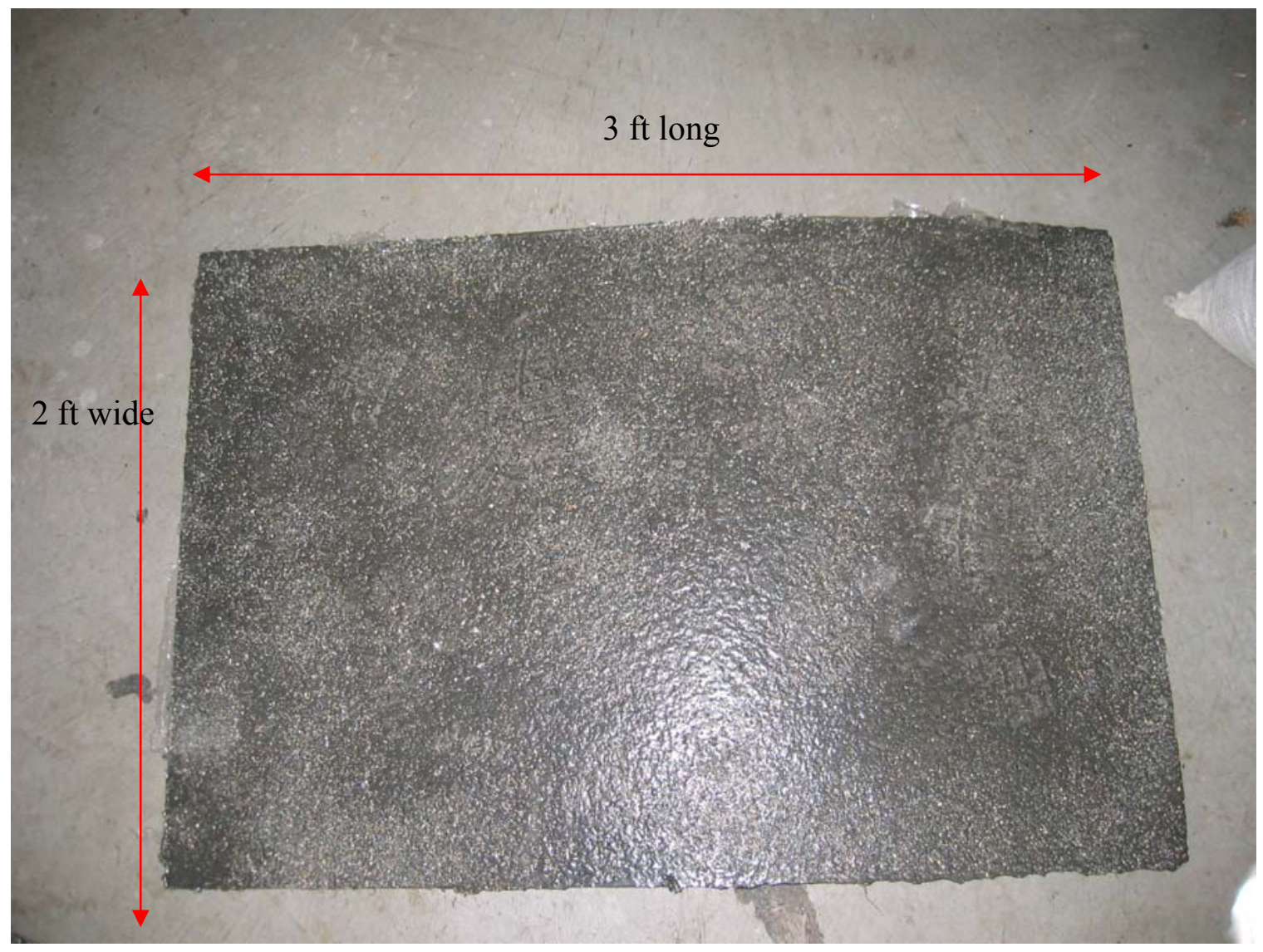

Figure 4.8: Wearing surfaces module of dimensions 36" x 24" x 3/8" (914.4mm x 609.6mm x 9.525mm).

Aluminum plate of various sizes and thicknesses were used to create "voids" at the bottom of the wearing surface modules. The aluminum plates were first placed on a flat surface and the wearing surface was overlaid on the top, with removable mold holding the wearing surface from the side. Use of plastic wrap under the wearing surface modules ensured that the modules did not stick to any surface, thus making them reusable for the laboratory experiments. Once the wearing surface cured, the aluminum plates were removed to create "voids" underneath the wearing surface. These voids were used later as air-filled debonds, or served as water-filled debonds by attaching a water pouch made using Glad "Press'N Seal" wraps. Due to the self-adhesive nature of the wraps, the 
pouch could be easily made by folding the wrap into half and gently pressing the sides together to seal off the edges (Figure 4.9). The size of the pouch ranged from as large as $12 " \mathrm{x} 12 "(304.8 \mathrm{~mm} \times 304.8 \mathrm{~mm})$ to as small as 1 " $\mathrm{x} 1$ " $(25.4 \mathrm{~mm} \times 25.4 \mathrm{~mm})$. The pouches were made into three thicknesses: $0.75 \mathrm{~mm}, 1.5 \mathrm{~mm}, 2.25 \mathrm{~mm}$ (Figure 4.10 ). These pouches were then filled with water using a syringe (Figure 4.11). While calculations were made to inject the exact amount of water to fill the entire void, some air bubbles were trapped inside the water pouch as seen in Figure 4.10, which was due to some human error. The bottom side of a typical wearing surface module with simulated debonds is shown in Figure 4.12.

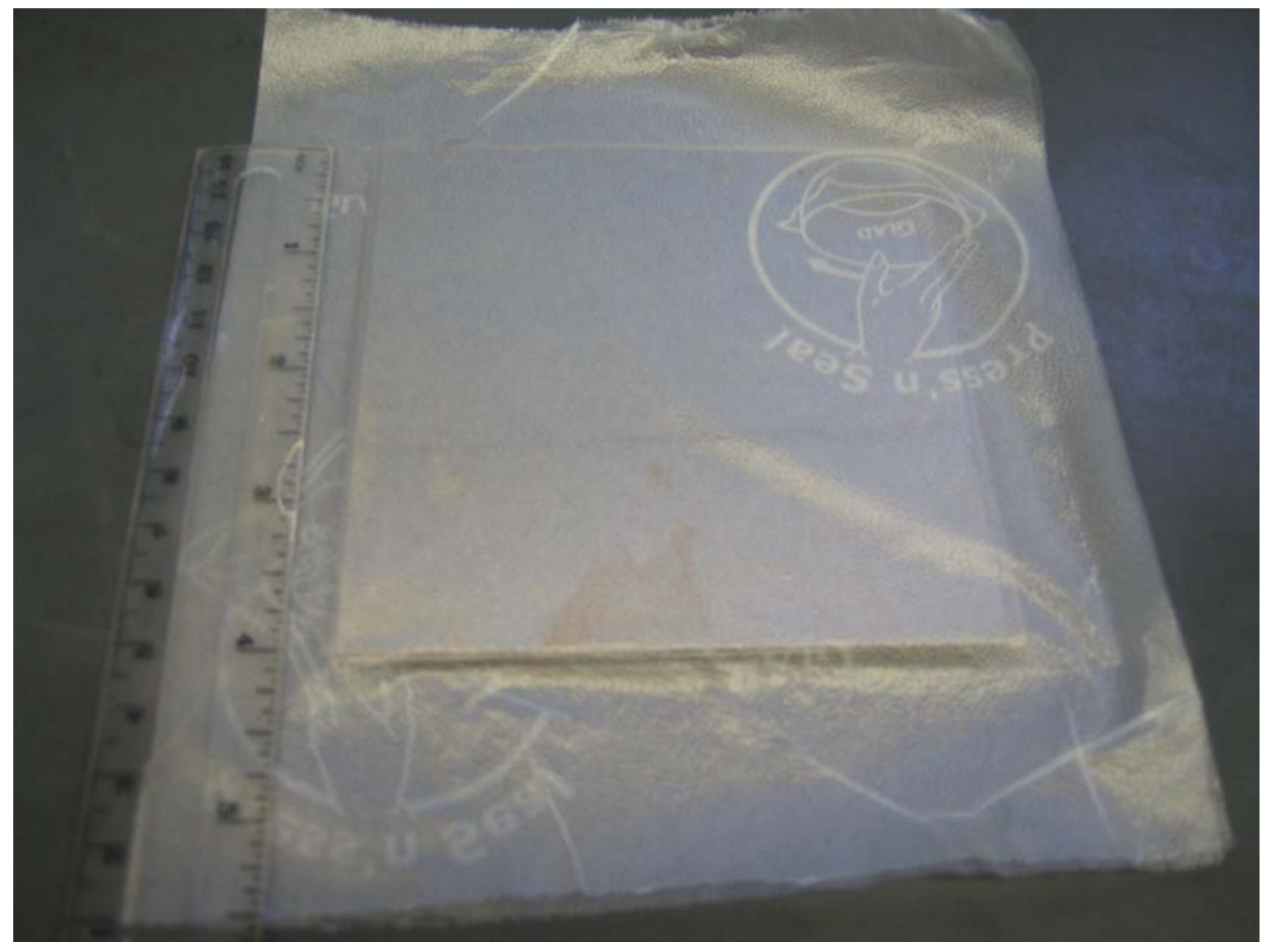

Figure 4.9: Pouch made using Press'N Seal Glad wrap. 


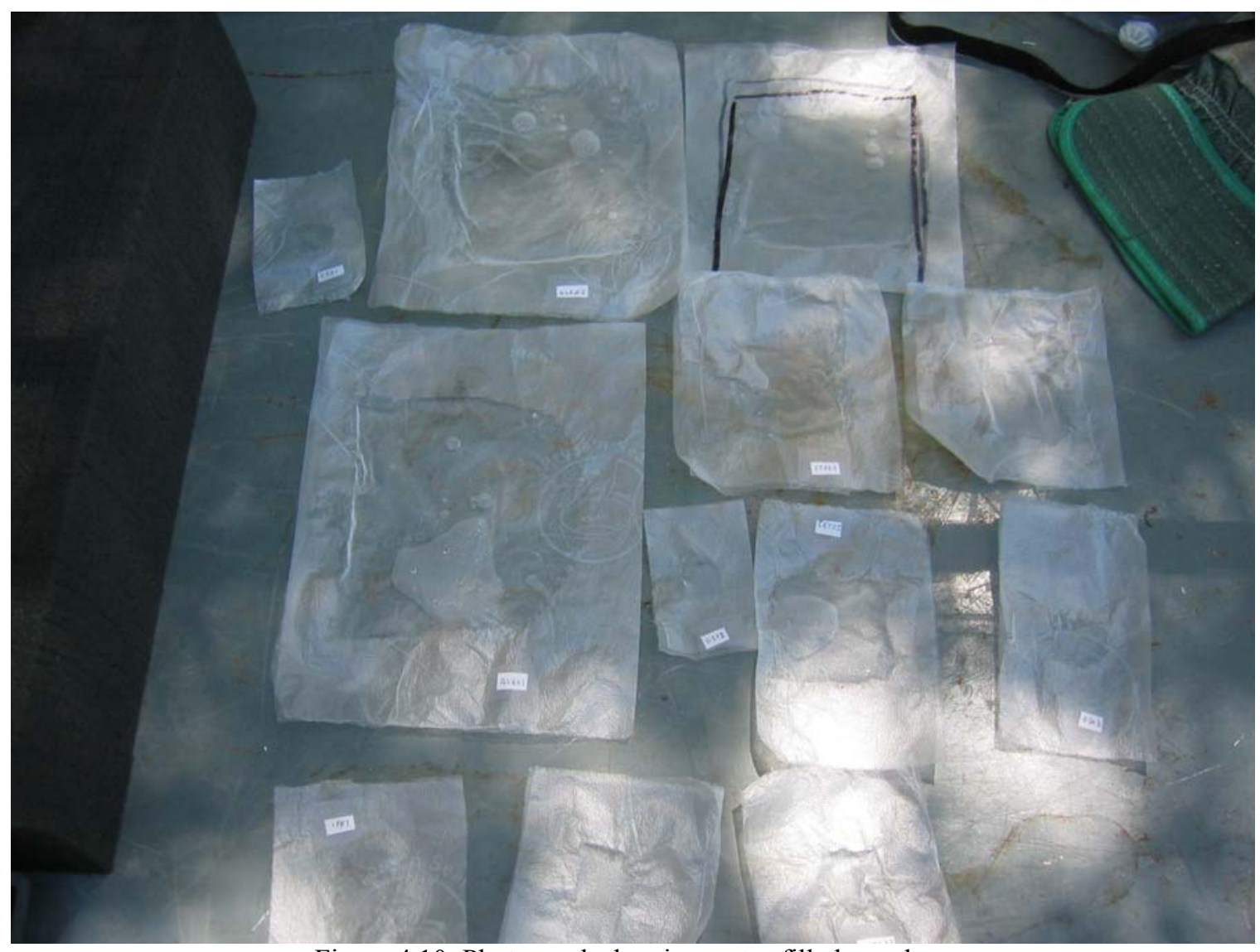

Figure 4.10: Photograph showing water-filled pouches.

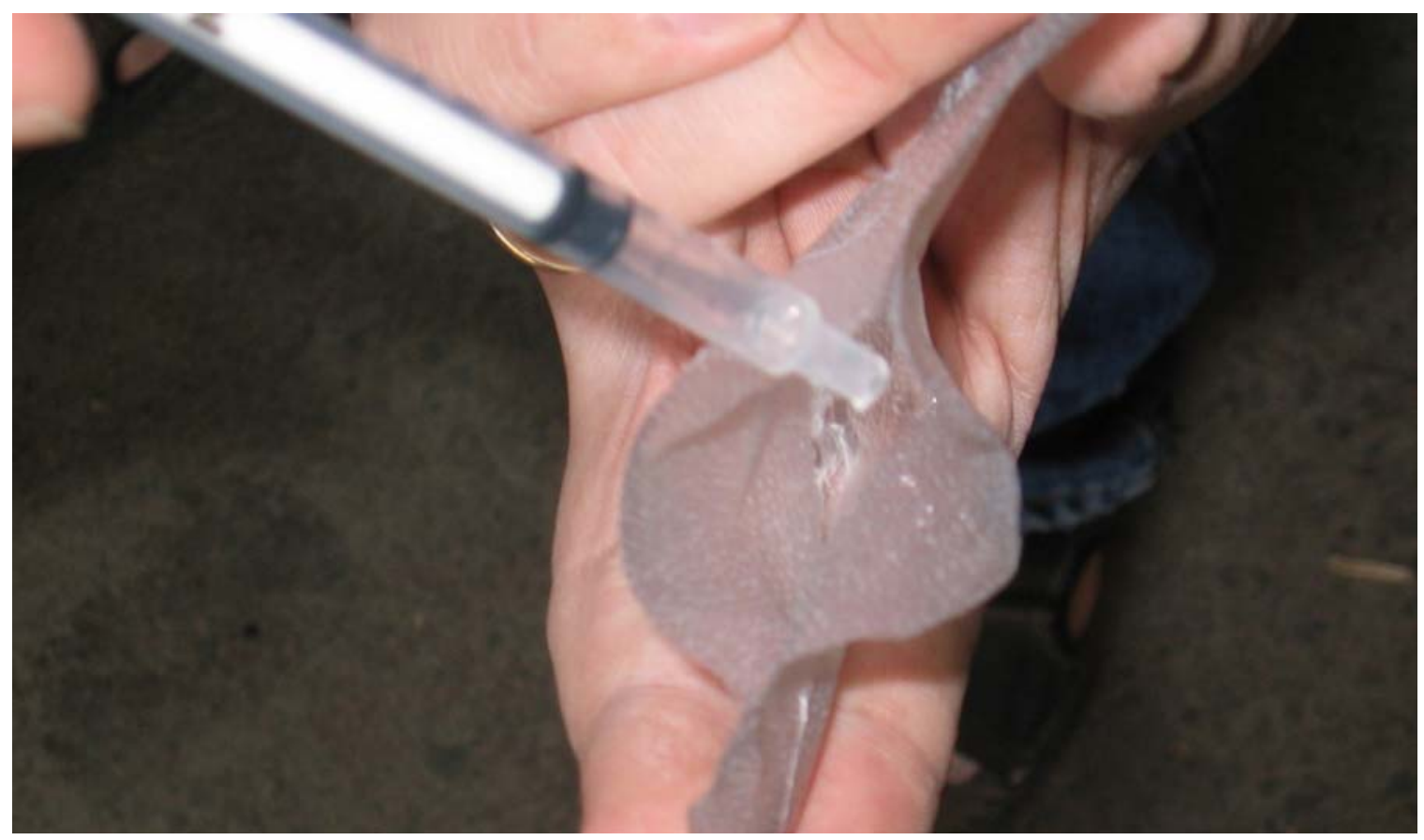

Figure 4.11: Glad pouch being filled with water using syringe. 


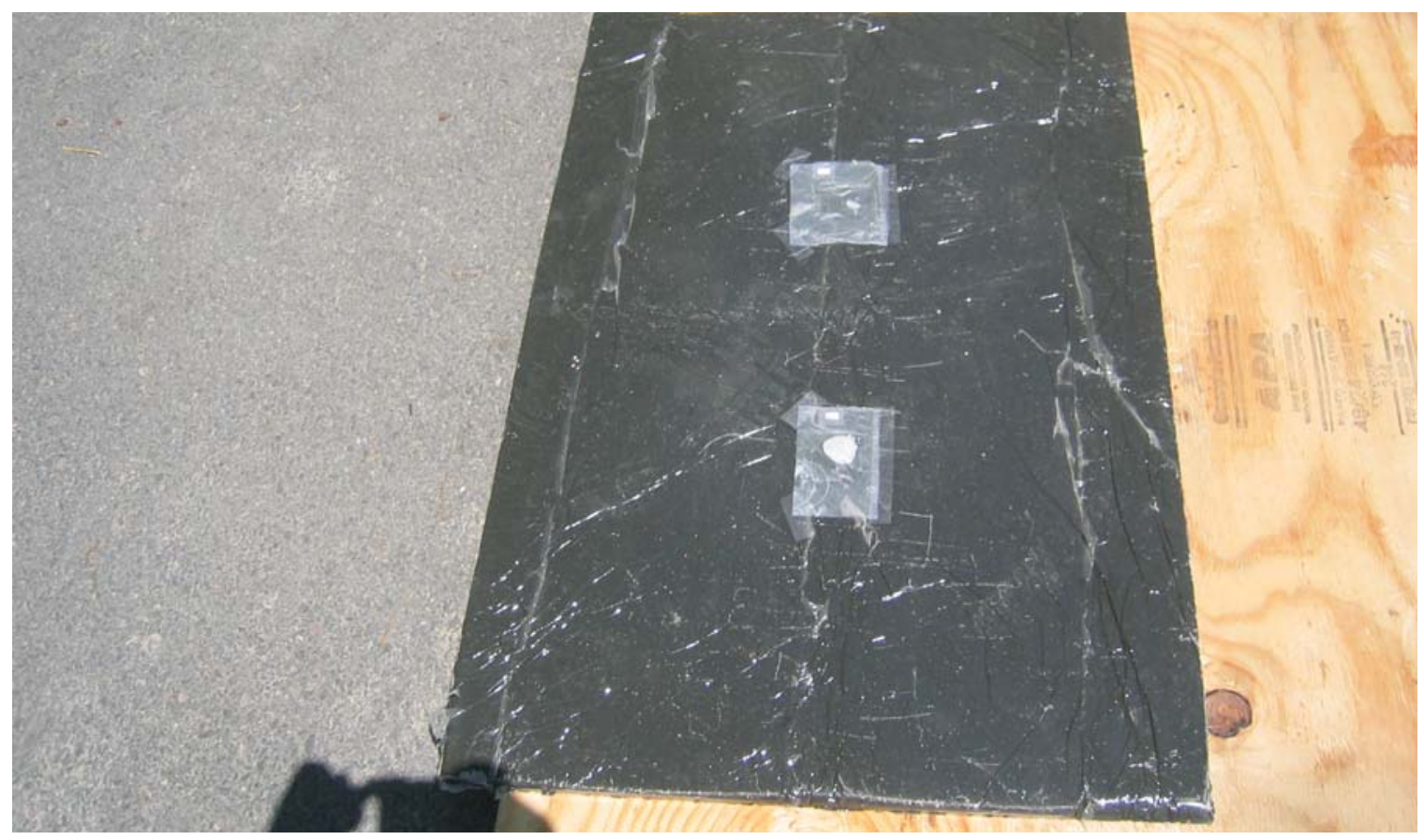

Figure 4.12: Bottom side of a wearing surface with two water-filled simulated debonds attached.

Along with the wearing surface modules, a delamination deck module was created (Figure 4.13). Two FRP bridge deck modules were first cut out of a large deck. The small modules were then brought to the shop to manually cut out voids to simulate delaminations. Two filling channels were cut out for simple refilling of the water delaminations later. The two modules were then joined together by applying appropriate amount of special glue used to bond the FRP module. The two delaminations prepared in this manner were of sizes 3"x3"x0.06" (76.2mm x 76.2mm x 1.5mm). One was air-filled and the other (connected to two filling channels) was water-filled. Figures $4.13-4.16$ illustrate the process of creating the two delaminations inside a deck module. 


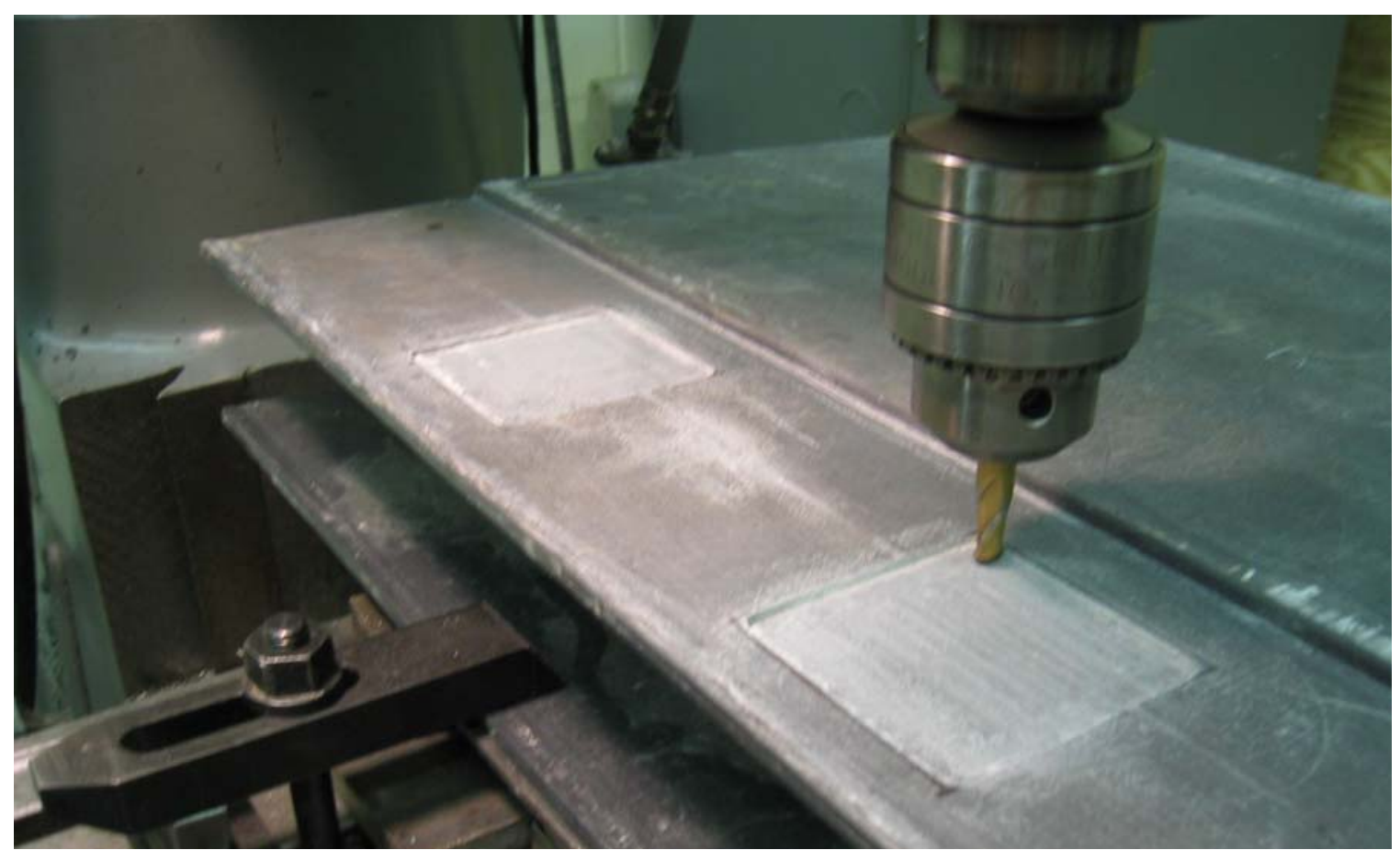

Figure 4.13: The machine drilling out voids to be used as delamination.

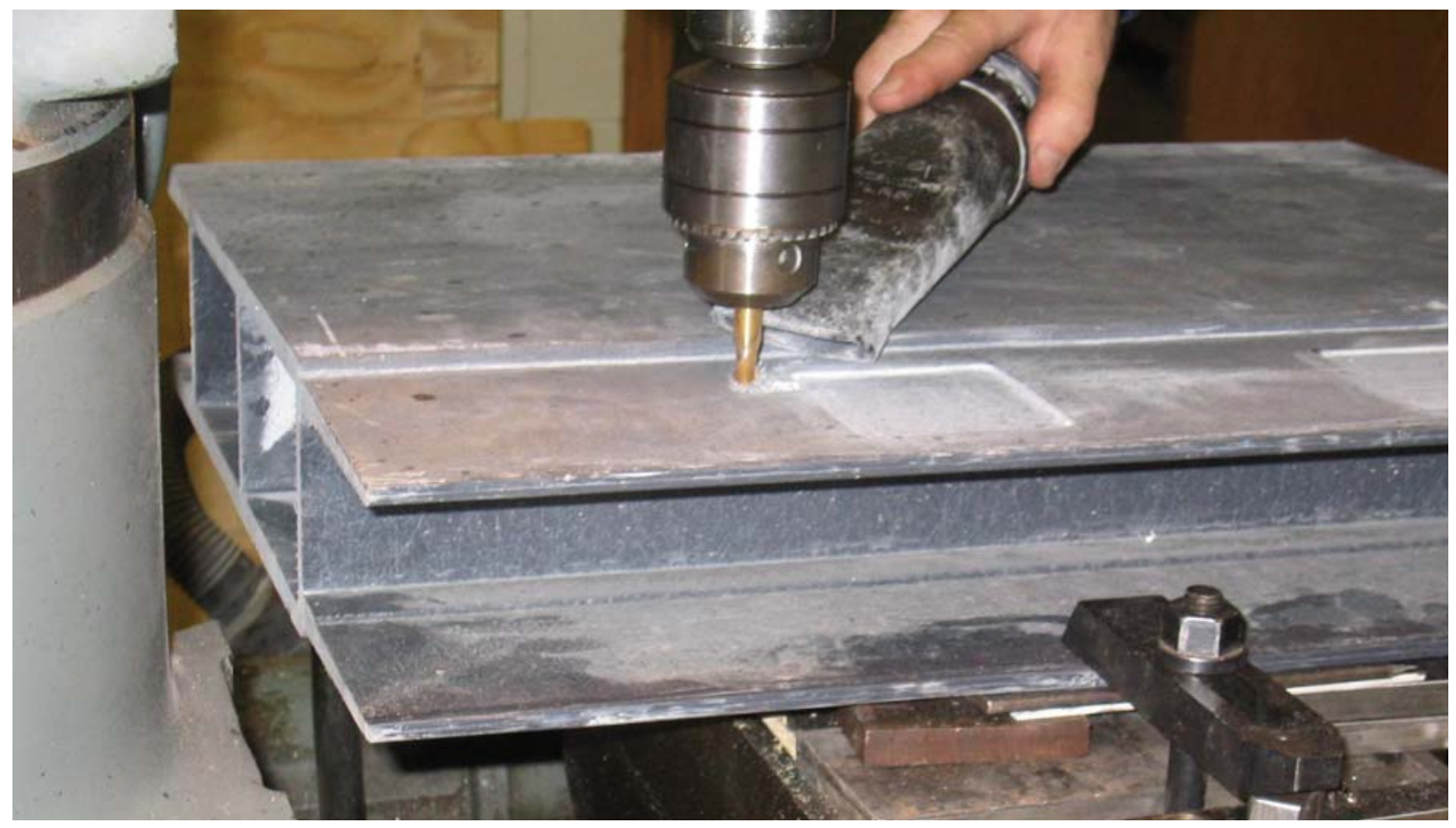

Figure 4.14: Cutting out the filling channel. 


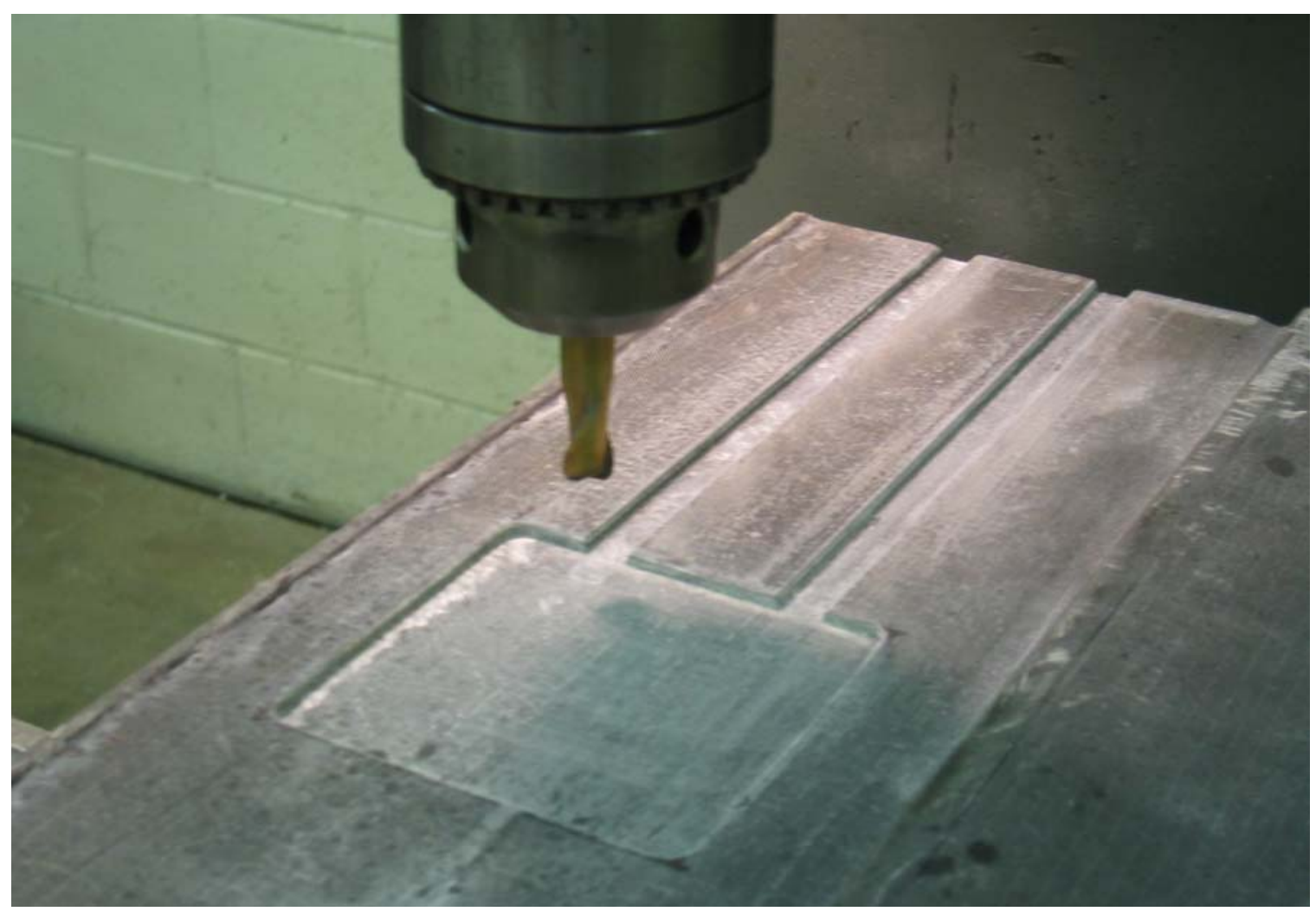

Figure 4.15: Two filling channels were cut out by the machine.

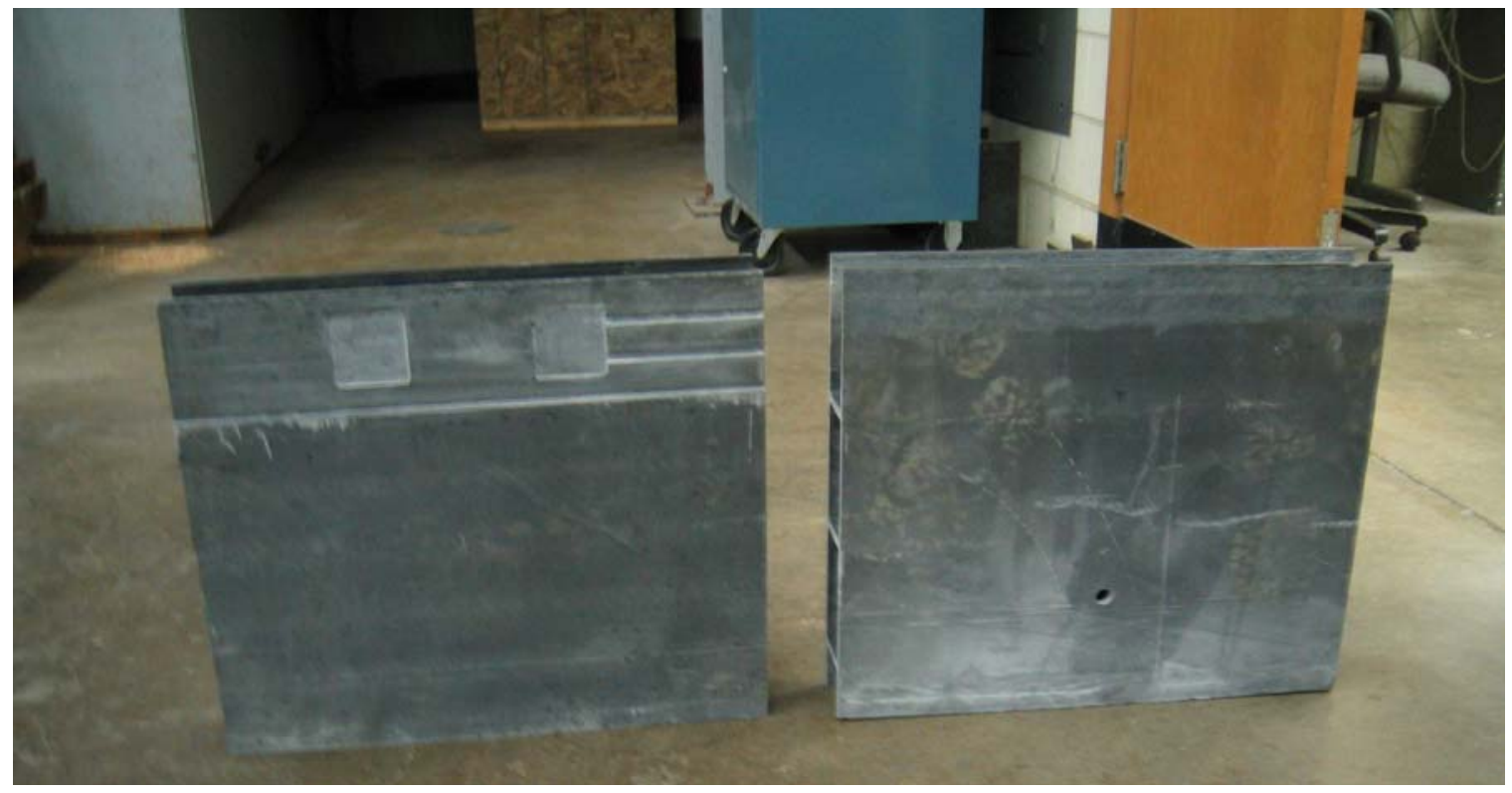

Figure 4.16: The two FRP modules to be joined together. 


\section{Chapter 5}

\section{EXPERIMENTAL RESULTS FROM INFRARED THERMOGRAPHY}

Previous studies have shown that commercially available heater is a useful tool to help find debonds as small as 1" x 1" $(25.4 \mathrm{~mm} \times 25.4 \mathrm{~mm})$ in small scale FRP bridge deck specimens (Halabe et al. 2004d). To further evaluate the feasibility of utilizing commercially available heating source in subsurface detection of defects on FRP bridge decks, similar tests were conducted on a larger scale specimen $\left(6^{\prime}\right.$ x $3^{\prime}$, or $1828.8 \mathrm{~mm}$ x 914.4mm). Furthermore, the same specimens were subjected to two rounds of solar radiation tests during two different seasons. The first solar radiation test was conducted during the early fall season. The abundance of solar energy and relatively calm wind yielded excellent heating of the FRP deck. The second solar radiation test was conducted during late winter/early spring season. The low ambient temperature provided a testing environment with low rate of heat transfer between the FRP bridge deck specimen and the surroundings. In addition to solar radiation tests, a heater and an electric heating blanket were used as active heat sources.

\subsection{IRT USING COMMERCIALLY AVAILABLE HEATER AS ACTIVE HEAT SOURCE}

Uniform heating was achieved by placing a $1500 \mathrm{~W}$ heater few inches away from the wearing surface and moving the heater back and forth repeatedly to ensure no overheating of one region. Heat was applied for about 5 minutes. The infrared image of 
the specimen revealed the location of the air-filled debonds as white regions. They were areas with higher temperature when compared to the surrounding defect-free regions. The color scale showing the temperature variability over the deck area is also available with the infrared image. A simple post-processing step was used in which the infrared image's scale was adjusted until a clear contrast could be seen. Figure 5.1 shows the processed infrared image of a 4" x 4" x 0.09" (101.6mm x 101.6mm x 2.25mm) air-filled debond.

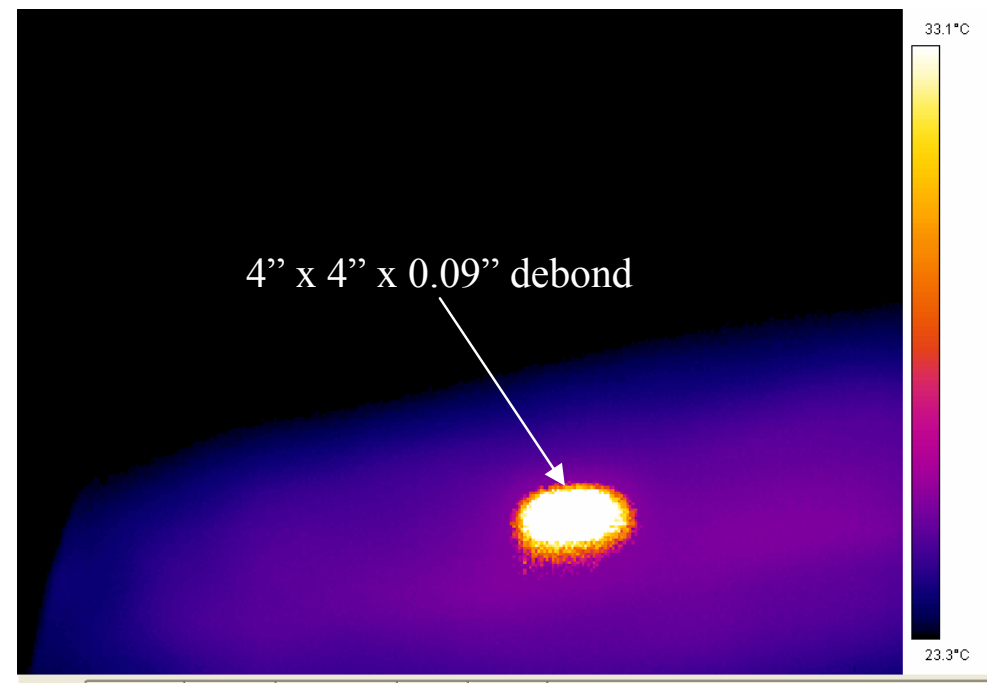

Figure 5.1: Infrared image of 4" x 4" x 0.09" (101.6mm x 101.6mm x 2.25mm) air-filled debond.

By repeating the same procedure, it was found that air-filled debond as small as $2 "$ × 2" x $0.06 "(50.8 \mathrm{~mm} \times 50.8 \mathrm{~mm} \times 1.5 \mathrm{~mm})$ could be detected by infrared thermography (Figure 5.2). 


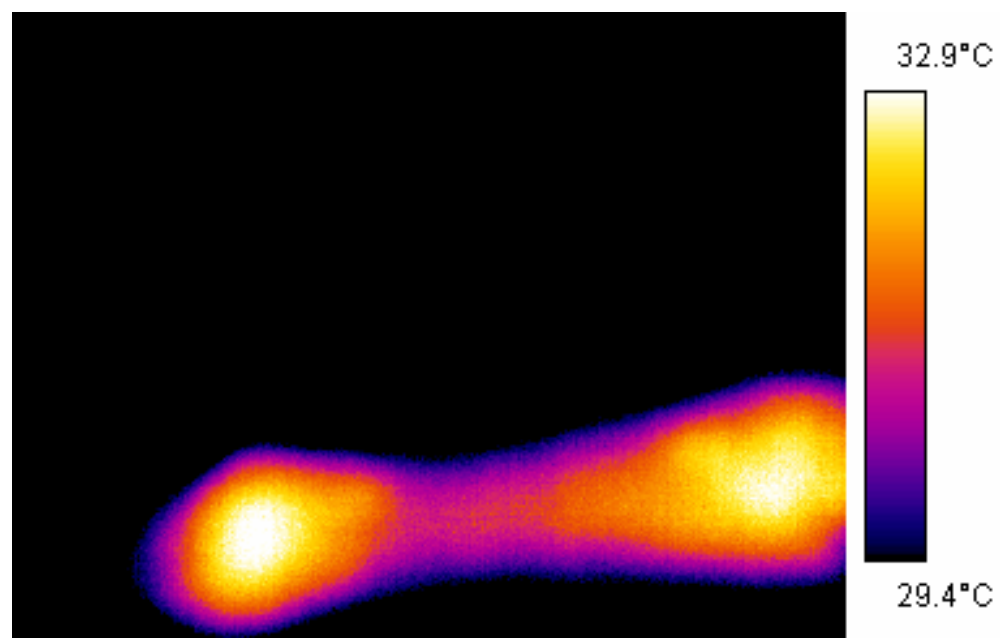

Figure 5.2: Debonds detected using infrared image.

In this testing, infrared thermography was unable to detect the water-filled debonds, even though past research using small FRP deck specimens indicated that water-filled debonds as small as $1 / 16 "(1.6 \mathrm{~mm})$ in thickness could be detected using infrared thermography (Halabe et al. 2004d). This is because past testing used FRP deck specimens with small plan sizes ( $1^{\prime}$ x $2^{\prime}=2$ sq. ft.), which allowed intense heat to be applied to the deck surface using a commercially available $1500 \mathrm{~W}$ heater. On the other hand, the current test deck had a much larger plan size $\left(3^{\prime} \times 6^{\prime}=18\right.$ sq. ft.), which was needed for GPR testing. However, the larger plan size required the heater to be moved around to cover a much larger area, thus reducing the heat intensity on the deck. 


\subsection{IRT USING SOLAR RADIATION AS ACTIVE HEAT SOURCE}

\subsubsection{Solar Radiation Test Conducted in September 2005}

The solar radiation test was conducted on a sunny day, with ambient temperature with highs in the $70 \mathrm{~s} F(\cong 22 \mathrm{C})$. The bridge deck module with wearing surfaces (with debonds) and another module with defect-free wearing surface (with delamination) were placed directly under the sun for solar radiation heating. Each wearing surface module consisted of several water-filled and air-filled defects. The exact location of the defects are shown in Figures 5.3 and 5.4. The modules were placed in the middle of an open space which had no obstruction to sunlight (Figure 5.5 and Figure 5.6). The solar radiation test started at 9 A.M. and lasted for 24 hours. Readings were taken once every half hour for the first 12 hours. A reading was taken for each subsequent one hour span.
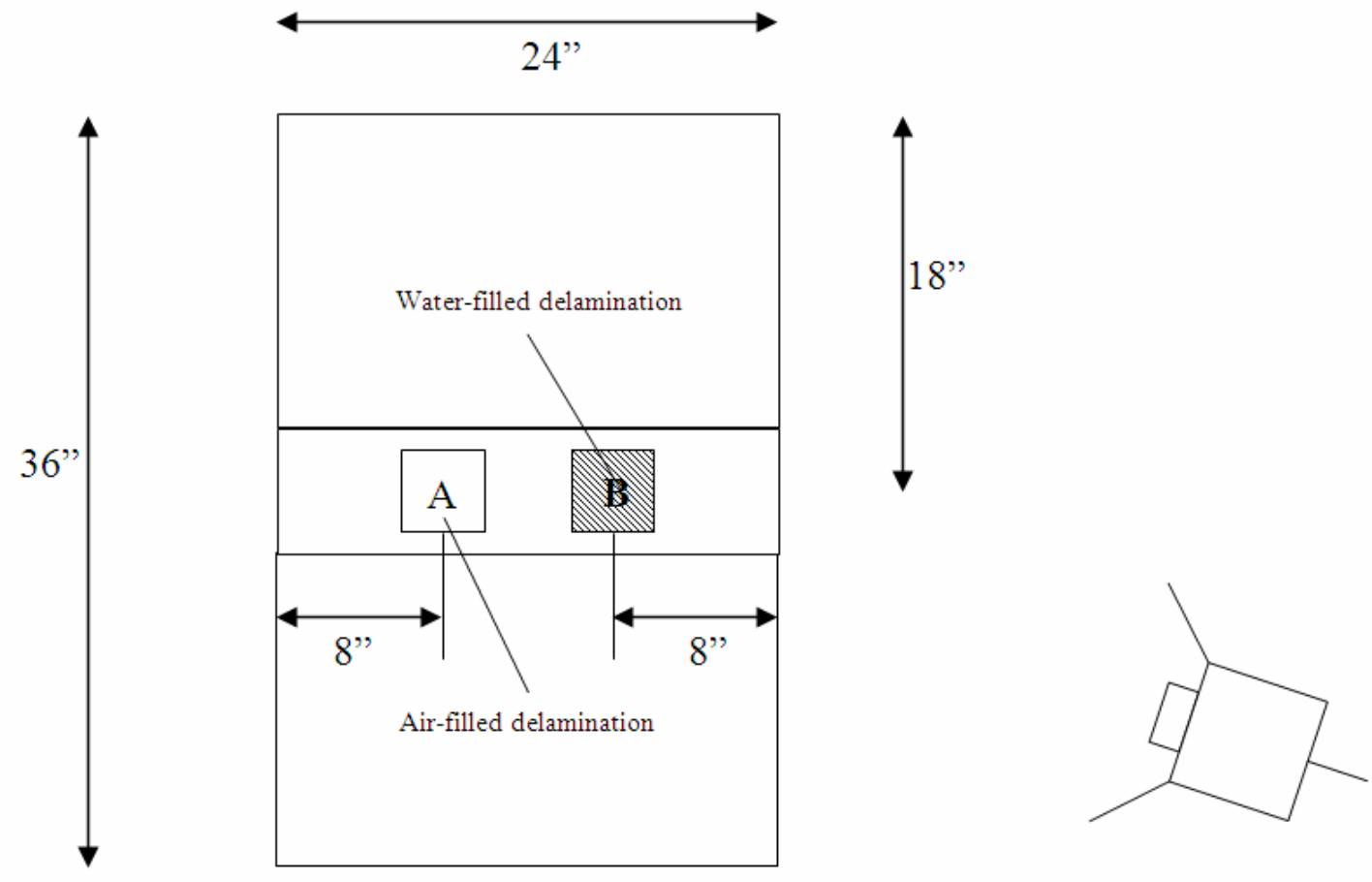

Figure 5.3: Defects map of delamination module for IRT solar radiation experiment. 

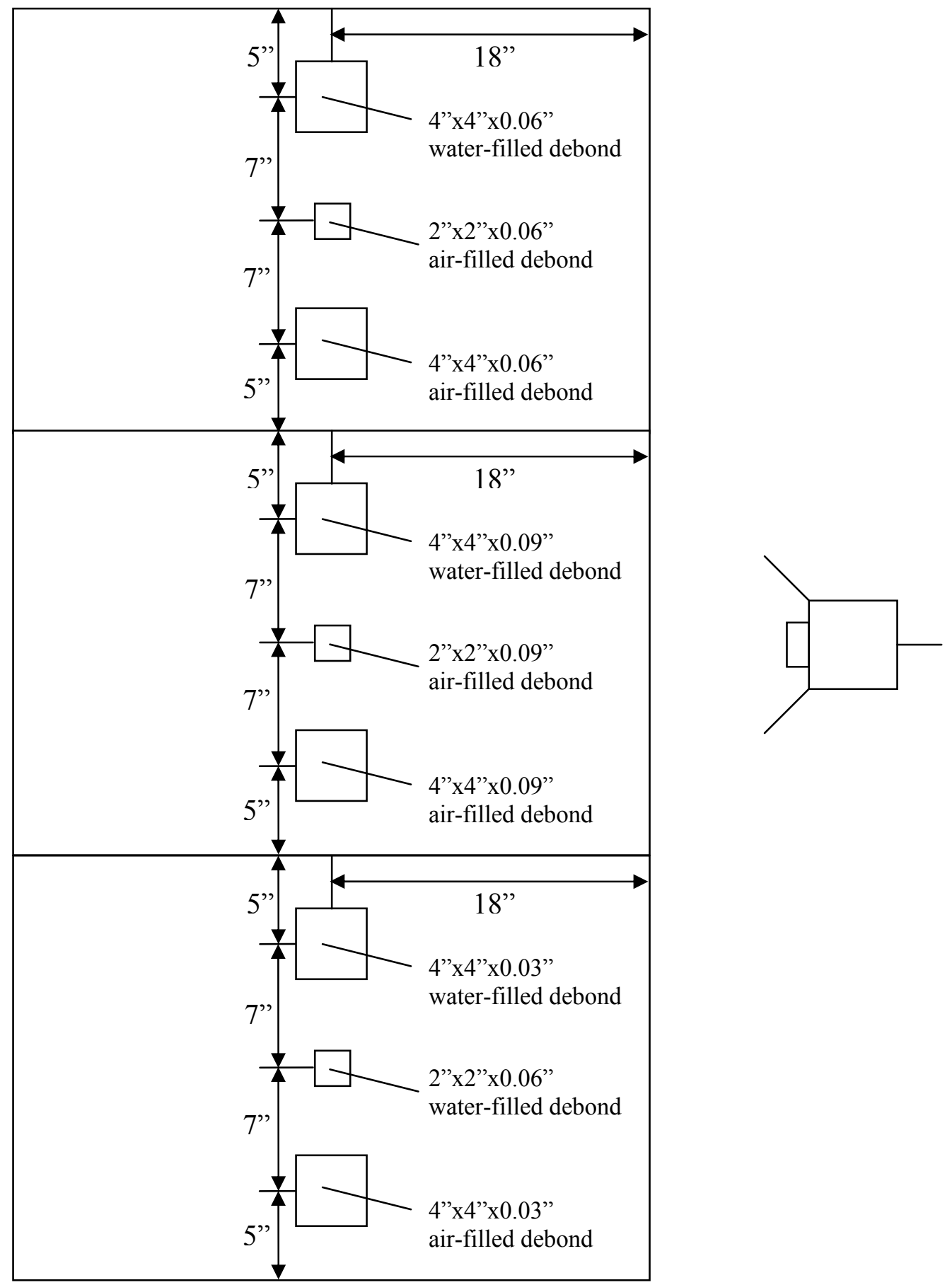

Figure 5.4: Defects map of debond module for IRT solar radiation experiment. 


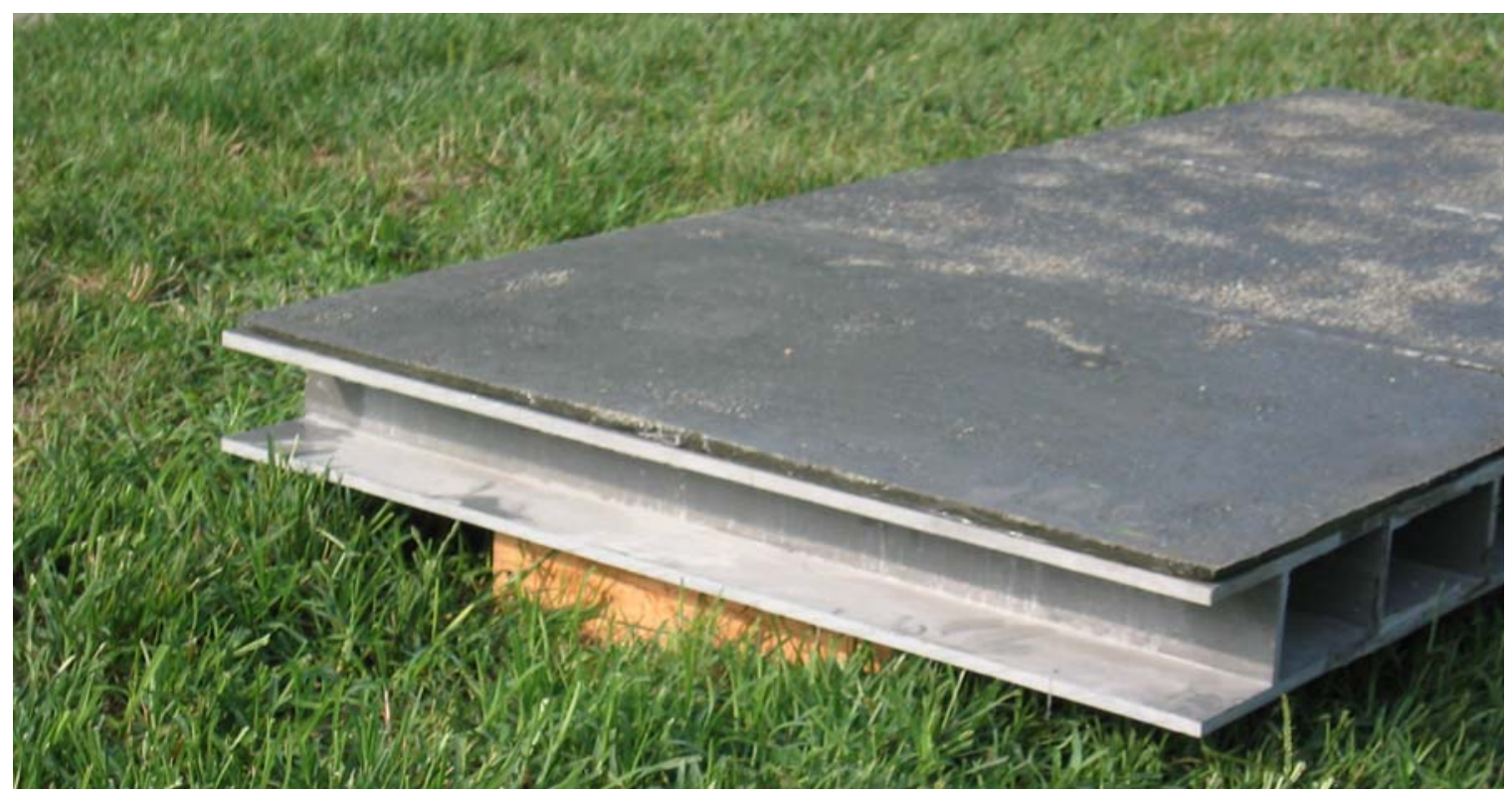

Figure 5.5: The bridge deck module with wearing surfaces.

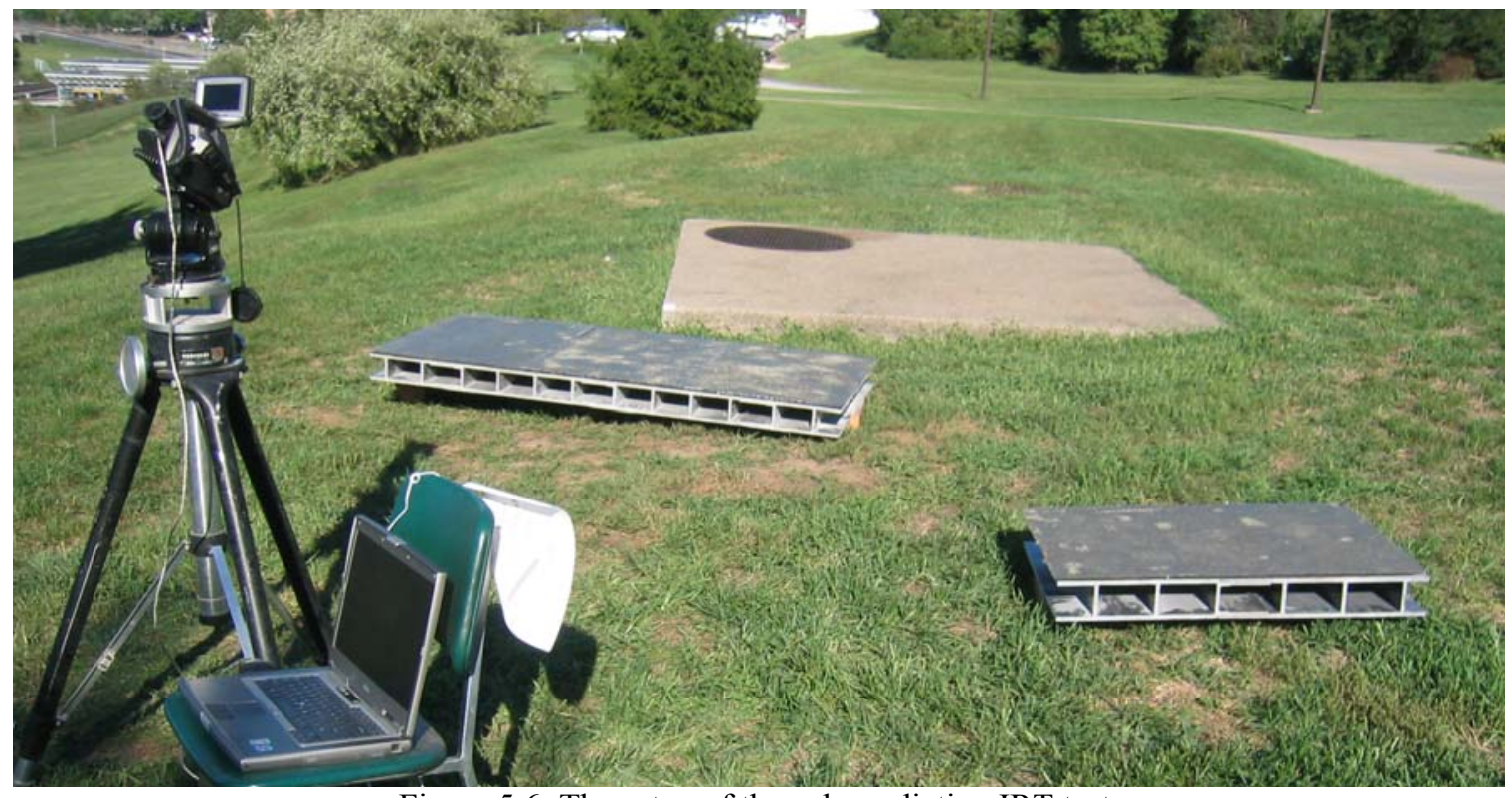

Figure 5.6: The setup of the solar radiation IRT test.

The data were processed through ThermaCAM ${ }^{\mathrm{TM}}$ Researcher software. As indicated in Figure 5.7, the solar radiation test did not reveal any detectable delaminations. Subsequent results confirmed that IRT is not capable of detecting any delamination, whether it's water or air-filled delamination. 


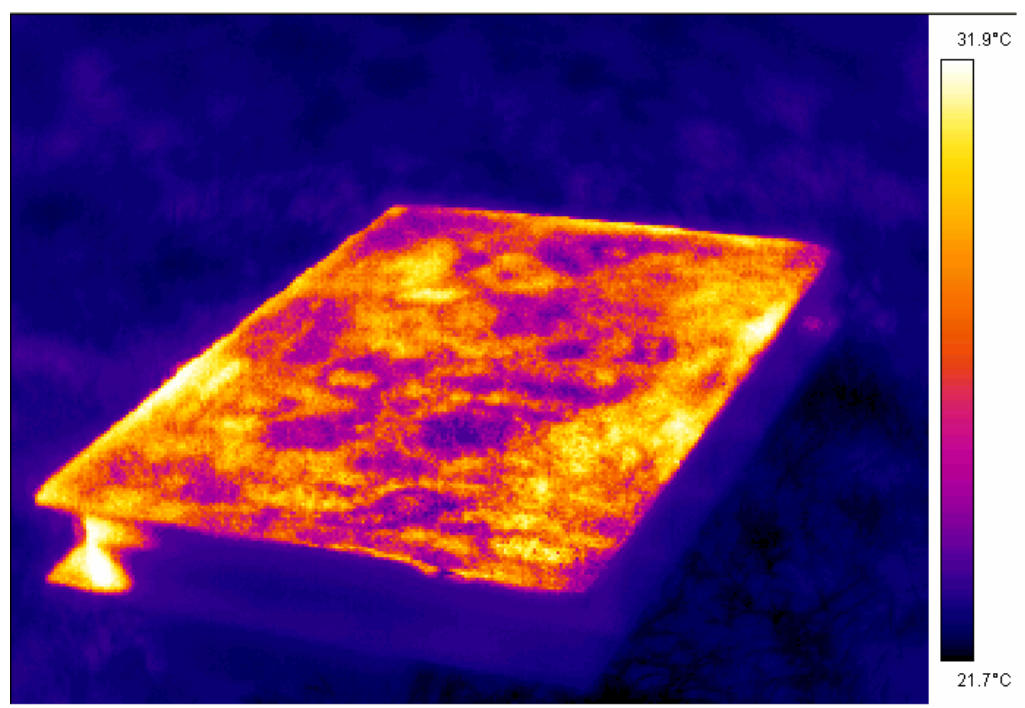

Figure 5.7: IRT image of FRP module with delaminations.

Figures 5.8 and 5.9 show the IRT images of the bridge deck module with debonds after two hours of solar heating. Evidently from Figure 5.8, the air-filled debonds were clearly detectable by IRT. The previous studies had shown that IRT test can comfortably detect air-filled debonds as small as $2 " \mathrm{x} 2$ 2" $(50.8 \mathrm{~mm} \times 50.8 \mathrm{~mm})$ and as thin as 0.06 " $(1.5 \mathrm{~mm})$. This study also showed that the air-filled debonds with thickness of 0.06 " $(1.5 \mathrm{~mm})$ and $0.09 "(2.25 \mathrm{~mm})$ and as small as $2 " \mathrm{x} 2 "(50.8 \mathrm{~mm} \times 50.8 \mathrm{~mm})$ in plan could be detected in the infrared images. However, as shown in Figure 5.9, the 4" x 4" x 0.03 " (101.6mm x $101.6 \mathrm{~mm} \times 0.75 \mathrm{~mm})$ air-filled debond and the water-filled debonds are not detectable by the IRT, which indicated that these 0.03 " $(0.75 \mathrm{~mm})$ thick debonds are too thin for detection using solar radiation. Further processed results indicated that the waterfilled debonds are not as clearly shown as the air-filled debonds. Figure 5.10 shows the IRT image taken after 5 hours of solar heating at 2:30 PM. The 4" x 4" x 0.09" $(101.6 \mathrm{~mm}$ $\mathrm{x} 101.6 \mathrm{~mm} \times 2.25 \mathrm{~mm}$ ) water-filled debond is visible along with all the air-filled debonds. Usually, a water-filled defect should show up as a cold-spot as opposed to the indicated 
hot-spot in Figure 5.10. Since the water-filled debond was heated for 5 hours, the water stored lot of heat energy due to its high specific heat capacity. Thus, it is understandable that the temperature would be higher after prolonged heating of water-filled defect, thus leading to a hot-spot as opposed to a normal cold-spot for a water-filled defect.

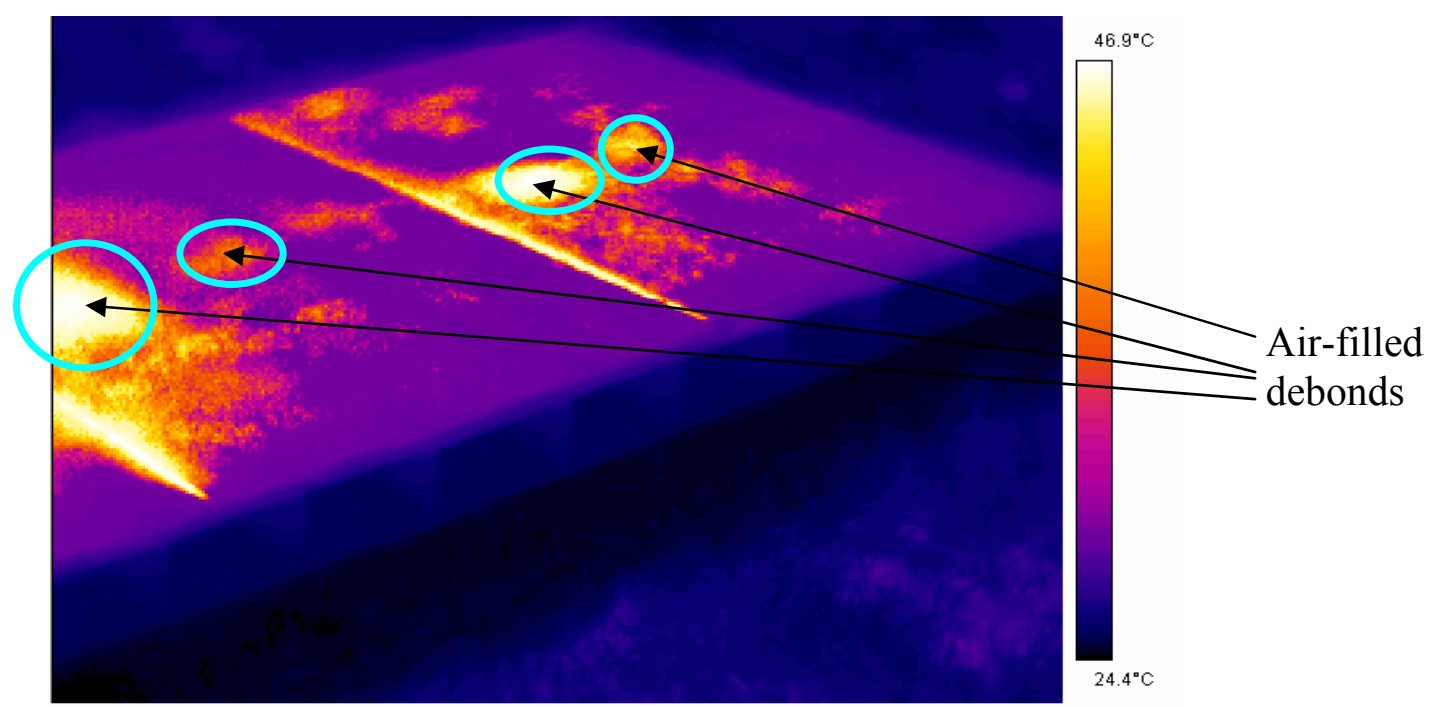

Figure 5.8: IRT image \#1 of the bridge deck at 11:30 AM.

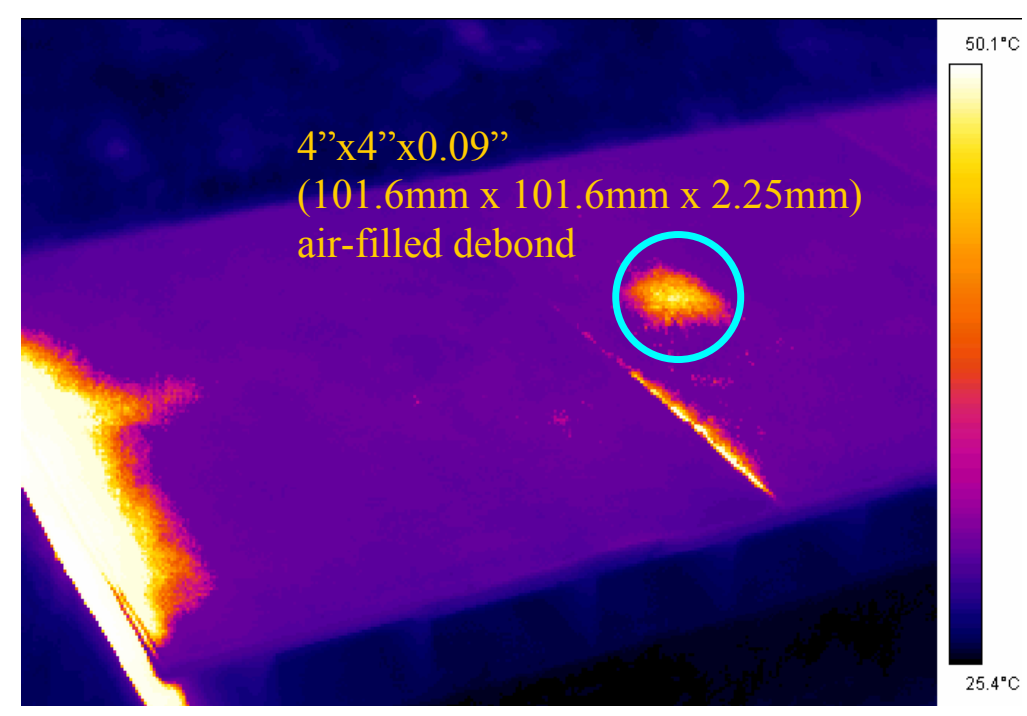

Figure 5.9: IRT image \#2 of the bridge deck at 11:30 AM. 


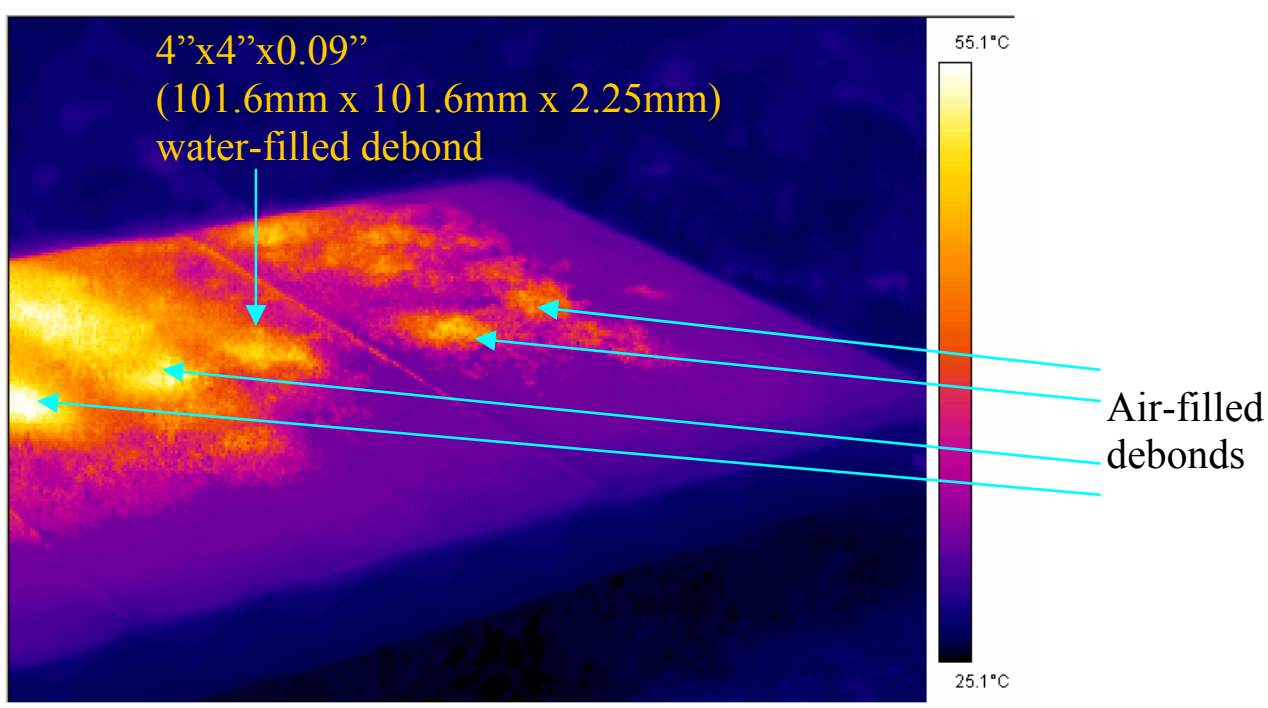

Figure 5.10: IRT image taken after 5 hours of solar heating at 2:30 PM.

The surface temperature of the debonds were recorded and plotted using the Microsoft Excel. As seen in Figure 5.11, the debonds are a few degrees hotter than the defect-free area during the day time. As the sun goes down, the effect of solar heating began to wear off. The temperatures converged around 5:30 PM and stayed relatively closed to each other throughout the night. The equilibrium state achieved during the night proved that cooling itself is not suitable for IRT detection of defects. On the other hand, the equilibrium state is a perfect stage for IRT experiment, since the temperatures are not fluctuating as much as it were during the day.

Figure 5.12 also shows the temperatures converging around 5:30 PM. The temperatures are fluctuating slightly more after 5:30 PM. for this wearing surface compared to the previous wearing surface data in Figure 5.11. This is due to the fact that the two wearing surfaces were made from two different batches of mixes. 


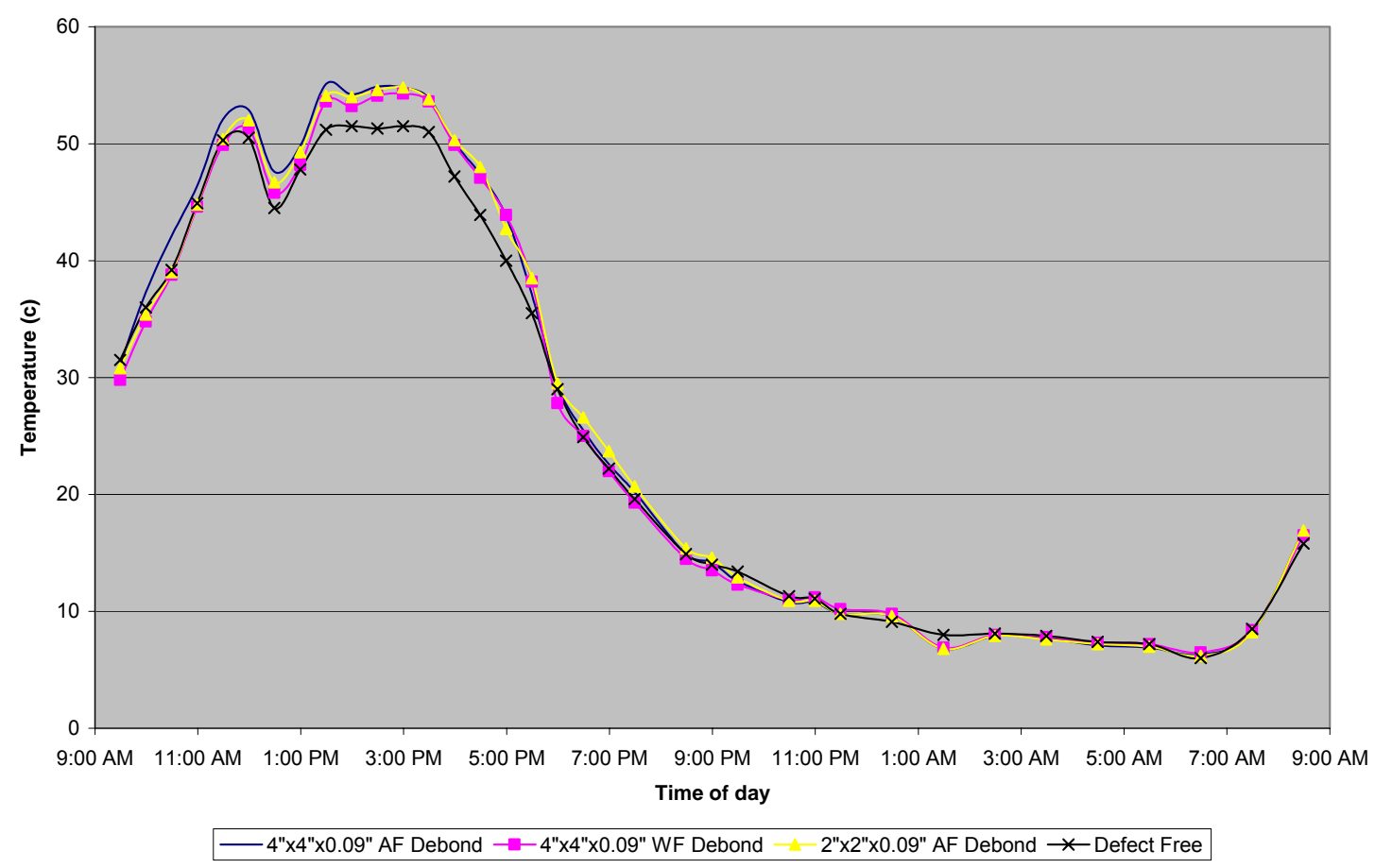

Figure 5.11: Surface temperature of defects versus defect-free area on wearing surface \#1.

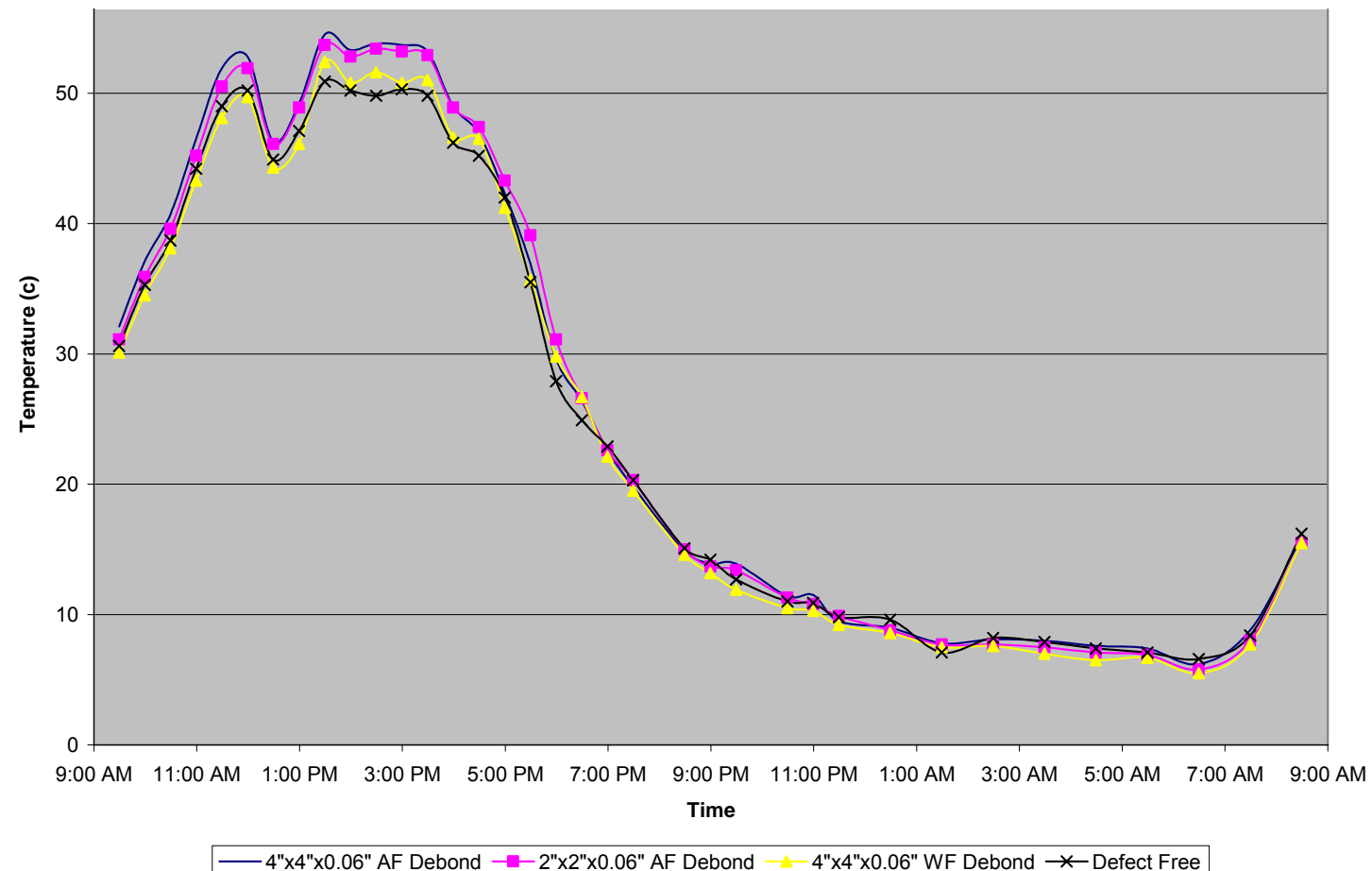

Figure 5.12: Surface temperature of defects versus defect-free area on wearing surface $\# 2$. 
Figure 5.13 and Figure 5.14(a) show the temperature differences for the previous data (from Figures 5.11 and 5.12). The temperature difference data was used for curvefitting using polynomial function to the $4^{\text {th }}$ order. From Figure 5.13 , it can be seen that the temperature differences are the highest between 12 PM to 5 PM. Also, it is clear that $4 " \times 4 " \times 0.09 "(101.6 \mathrm{~mm} \times 101.6 \mathrm{~mm} \times 2.25 \mathrm{~mm})$ air-filled defect has the highest temperature differences among all the defect sizes considered in this study, follow by 2 " x 2" x 0.09" (50.8mm x 50.8mm x 2.25mm) air-filled defect. The water-filled defect has the lowest temperature differences for wearing surface module \#1, as expected.

Since the source of the heating (sun) is the same for both wearing surfaces, and the fact that the defects embedded on each wearing surface are consistent relative to its defect-free area, all six detectable defects' temperature differences were plotted in Figure 5.14(a). Once again, it is clear that the temperature differences are the greatest between 12PM to 5PM. Also note that the 4" $\mathrm{P} 4$ " $\mathrm{x} 0.06 "(101.6 \mathrm{~mm} \times 101.6 \mathrm{~mm} \times 1.5 \mathrm{~mm})$ waterfilled debond's temperature differences are relatively low and thus very hard to distinguish from a regular IRT image. Notice that all the air-filled defects have higher maximum differences than any of the water-filled defects. It is consistent with the conclusion from previous studies. Furthermore, Figure 5.14(a) also indicated that an infrared experiment would obtain the best result if conducted during day time when the temperature differences between a defective and a defect-free area are the highest. However, since the temperature fluctuation is relatively low at night, it provides an ideal condition to conduct an infrared thermography test if an external active heat source is available. Figure 5.14(b) shows the entire set data collected during the test. Notice that the temperature variance at night (9PM to 6AM) is very low. 


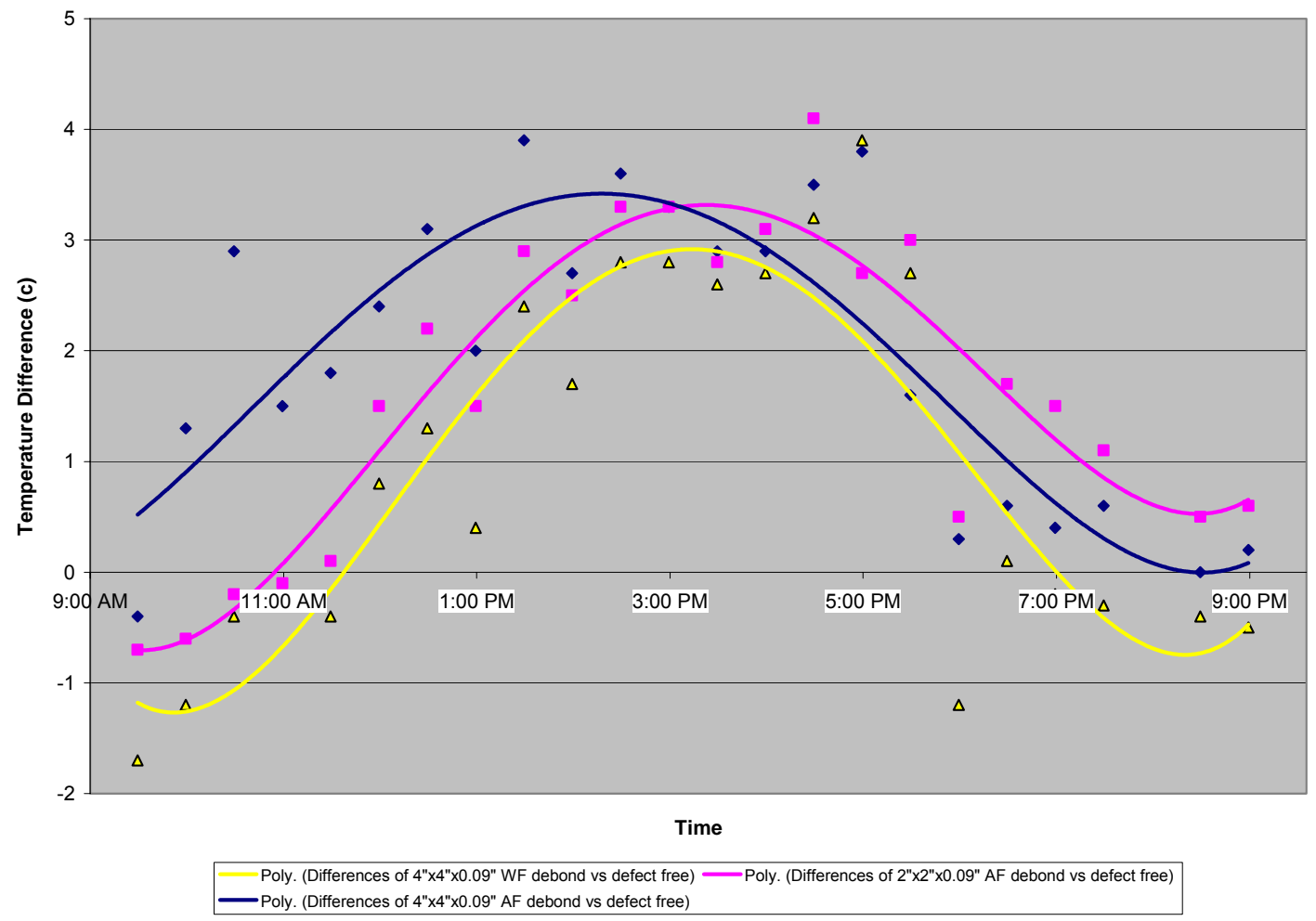

Figure 5.13: Temperature differences of various defects on wearing surface \#1.

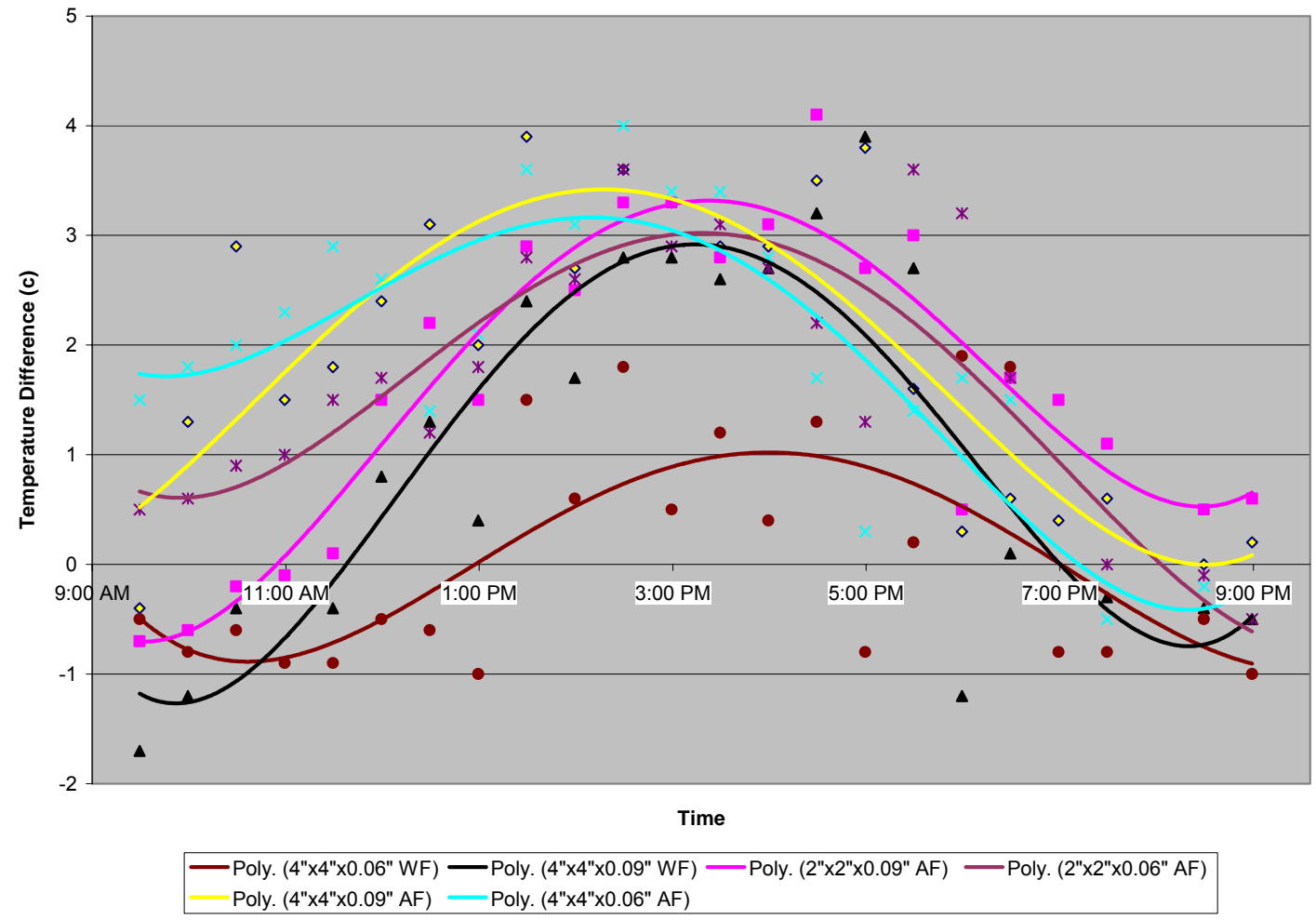

Figure 5.14(a): Temperature differences of various defects on all wearing surfaces. 


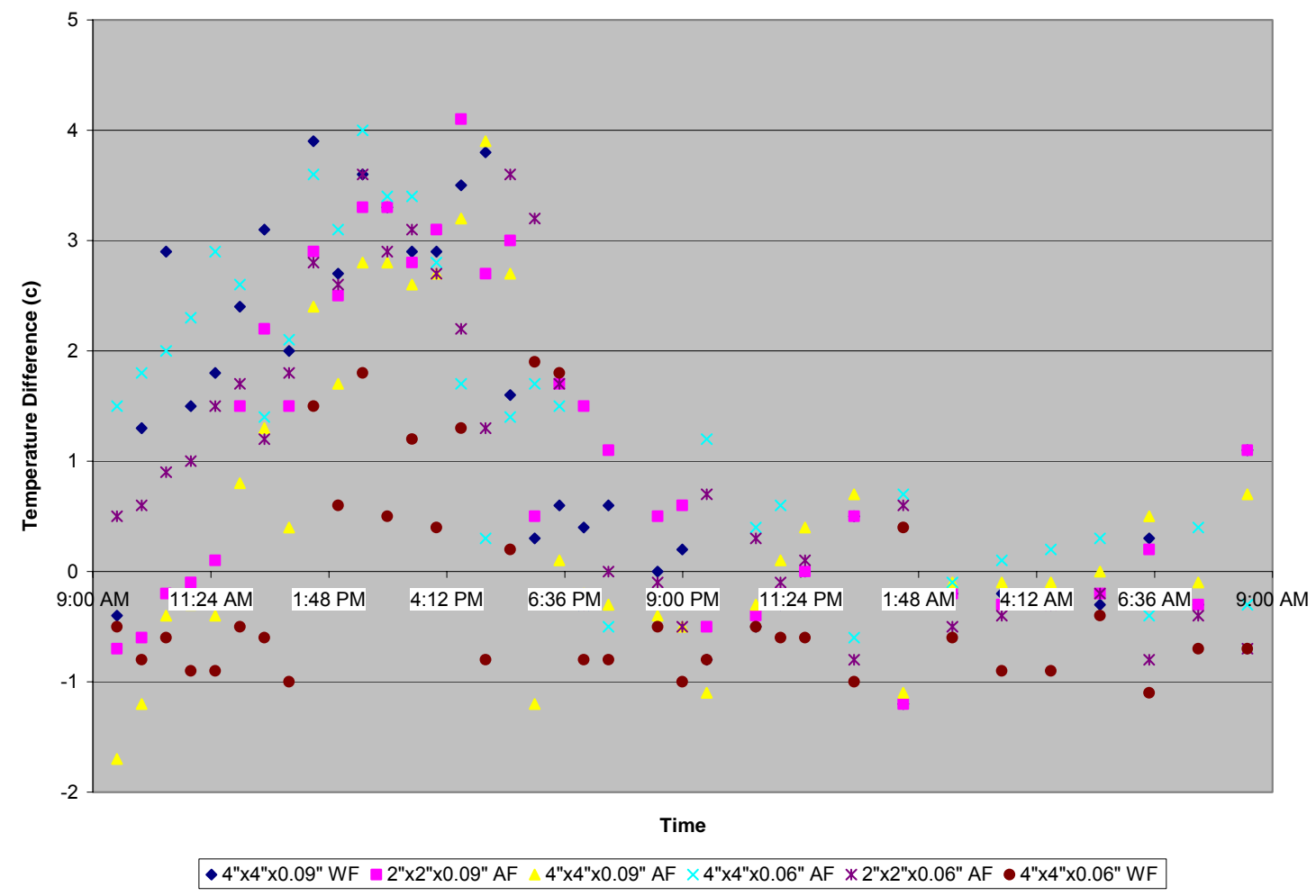

Figure 5.14(b): Temperature differences of various defects on all wearing surfaces, data only.

\subsubsection{Solar Radiation Test Conducted in March 2006}

The solar radiation test was conducted on a partly cloudy day, with ambient temperature highs in the $40 \mathrm{~s} \mathrm{~F} \mathrm{( \cong 4} \mathrm{C).} \mathrm{The} \mathrm{wind} \mathrm{was} \mathrm{blowing} \mathrm{at} 2-4 \mathrm{mph}$. The bridge deck module with wearing surfaces were placed directly under the sun for solar radiation heating. Each wearing surface module consisted of several water-filled and air-filled defects. The exact location of the defects are shown in Figure 5.18. The modules were placed in the middle of an open space which had no obstruction of sunlight. The solar radiation test started at $11 \mathrm{AM}$ and lasted for 4 hours. Readings were taken once every half hour.

Figure 5.15 and Figure 5.16 show the FRP bridge deck after being heated by the sun for 30 minutes. Figure 5.17 shows the FRP bridge deck after 3 hours of solar heating. 
Notice that the 4" x 4" x $0.09 "(101.6 \mathrm{~mm} \times 101.6 \mathrm{~mm} \times 2.25 \mathrm{~mm})$ air-filled debond can still be seen in Figure 5.17 while all other air-filled debonds disappeared. Furthermore, the appearances of the defects in Figure 5.15 and Figure 5.16 are not as prominent as those from the previous solar radiation test. More importantly, many of the smaller

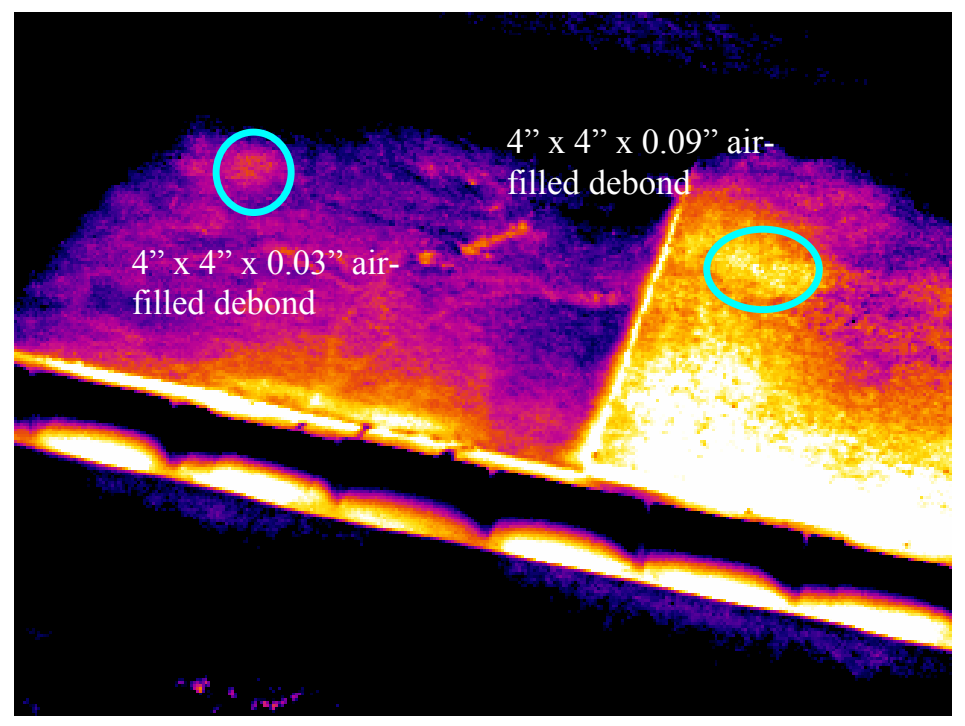

Figure 5.15: FRP bridge deck after 30 minutes of solar heating.

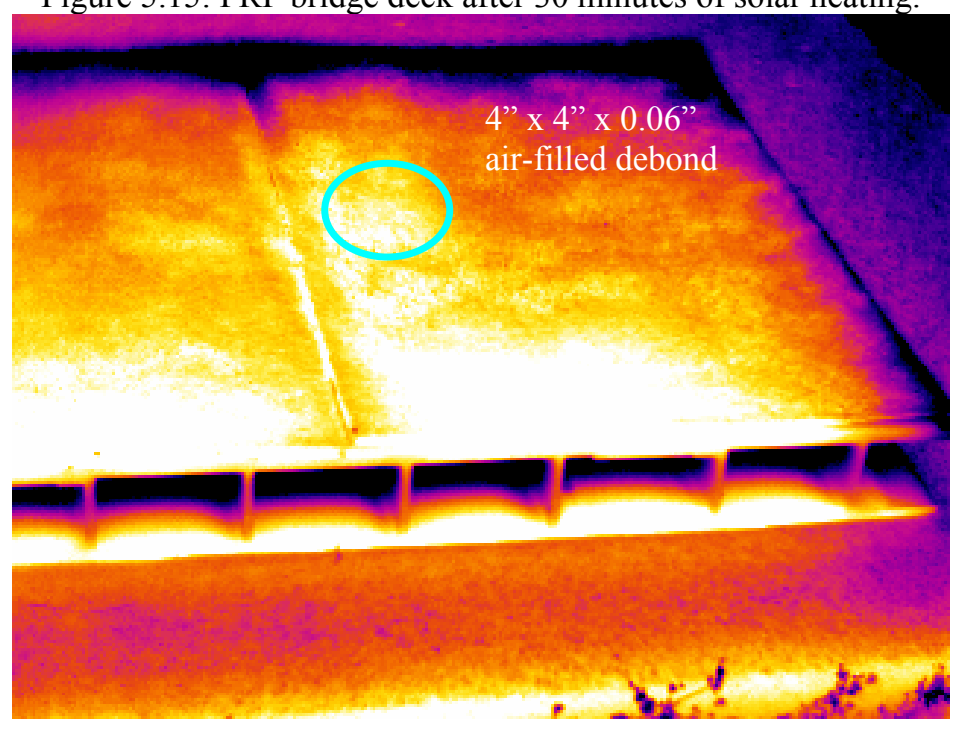

Figure 5.16: FRP bridge deck after 30 minutes of solar heating. 


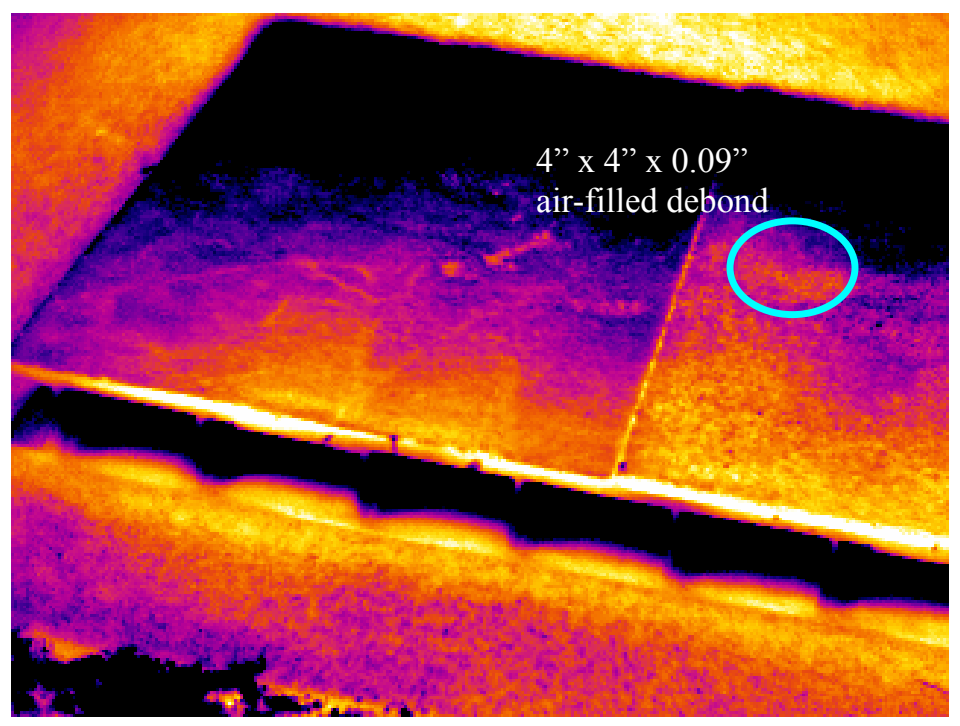

Figure 5.17: FRP bridge deck after 3 hours of solar heating.

defects that were detected by the previous solar radiation during a relatively warm September day could not be detected during the second solar radiation test in colder ambient temperature. Also, the wind flow was relatively strong compared to the September's solar radiation test, which reduced the temperature differences between defective and defect-free area. This explains the disappearance of the smaller defects as well as the vague appearance of the debonds that could be seen. 


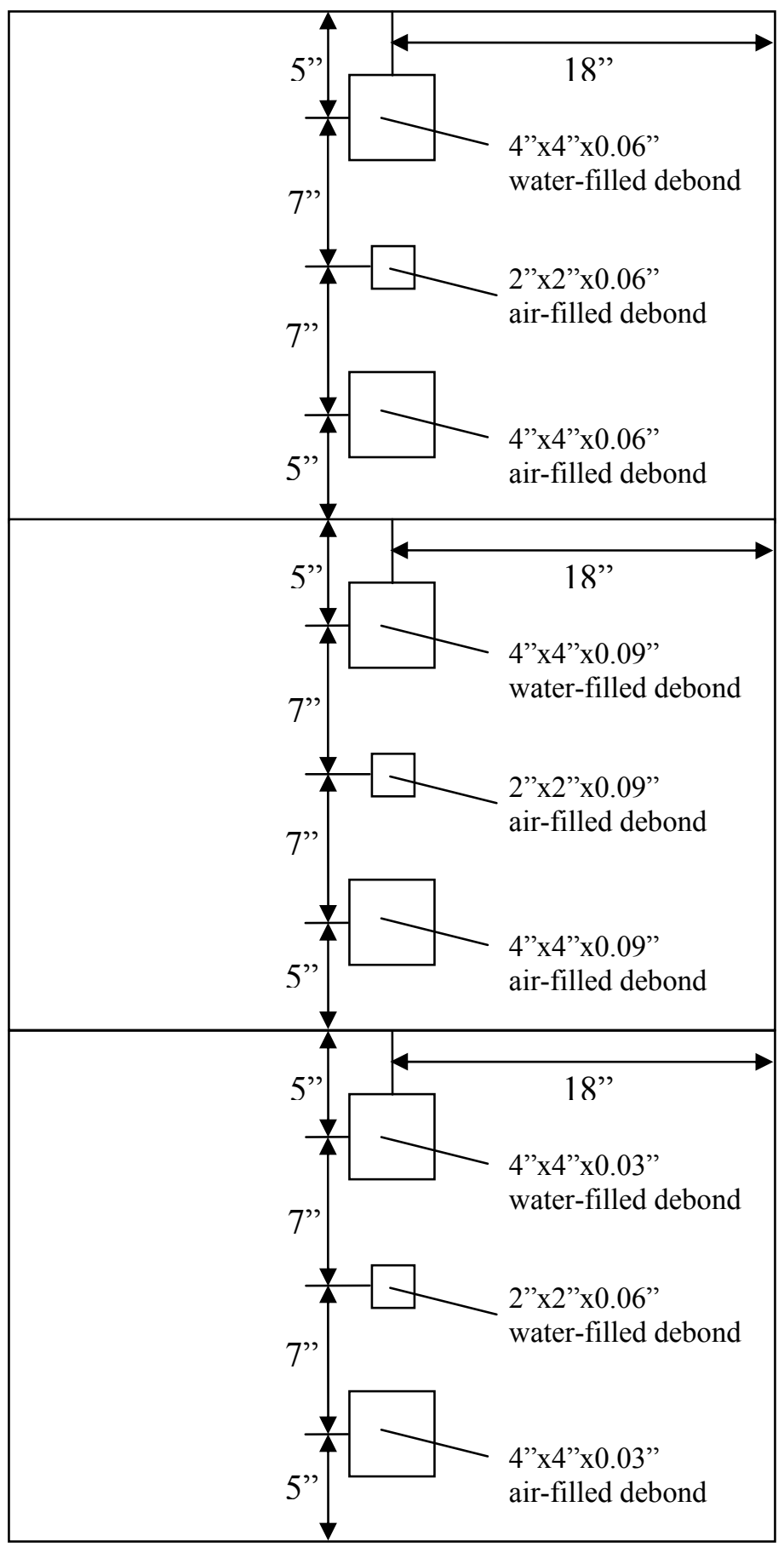

Figure 5.18: Defects map of debond module for IRT solar radiation experiment. 


\subsection{IRT USING HEATING BLANKET AS ACTIVE HEAT SOURCE}

The heating blanket IRT tests were conducted to determine the feasibility of using heating blanket as active heat source. The tests were conducted indoors, with an ambient temperature in the neighborhood of $61{ }^{\circ} \mathrm{F}\left(16^{\circ} \mathrm{C}\right)$. Figure 5.19 and Figure 5.20 show the experimental setup for the heating blanket IRT test. The wooden platforms surrounding the FRP bridge deck were for the GPR test prior to the IRT tests. The two grey color heating blankets were warmed up for 15 minutes prior to actual testing, to ensure the active heating was to be conducted at around $70^{\circ} \mathrm{F}$. As noted in Figures 5.19 and 5.20, the size of the heating blanket was not sufficient to cover up the entire bridge deck module, thus the data acquisition of the IRT was divided into two portions.

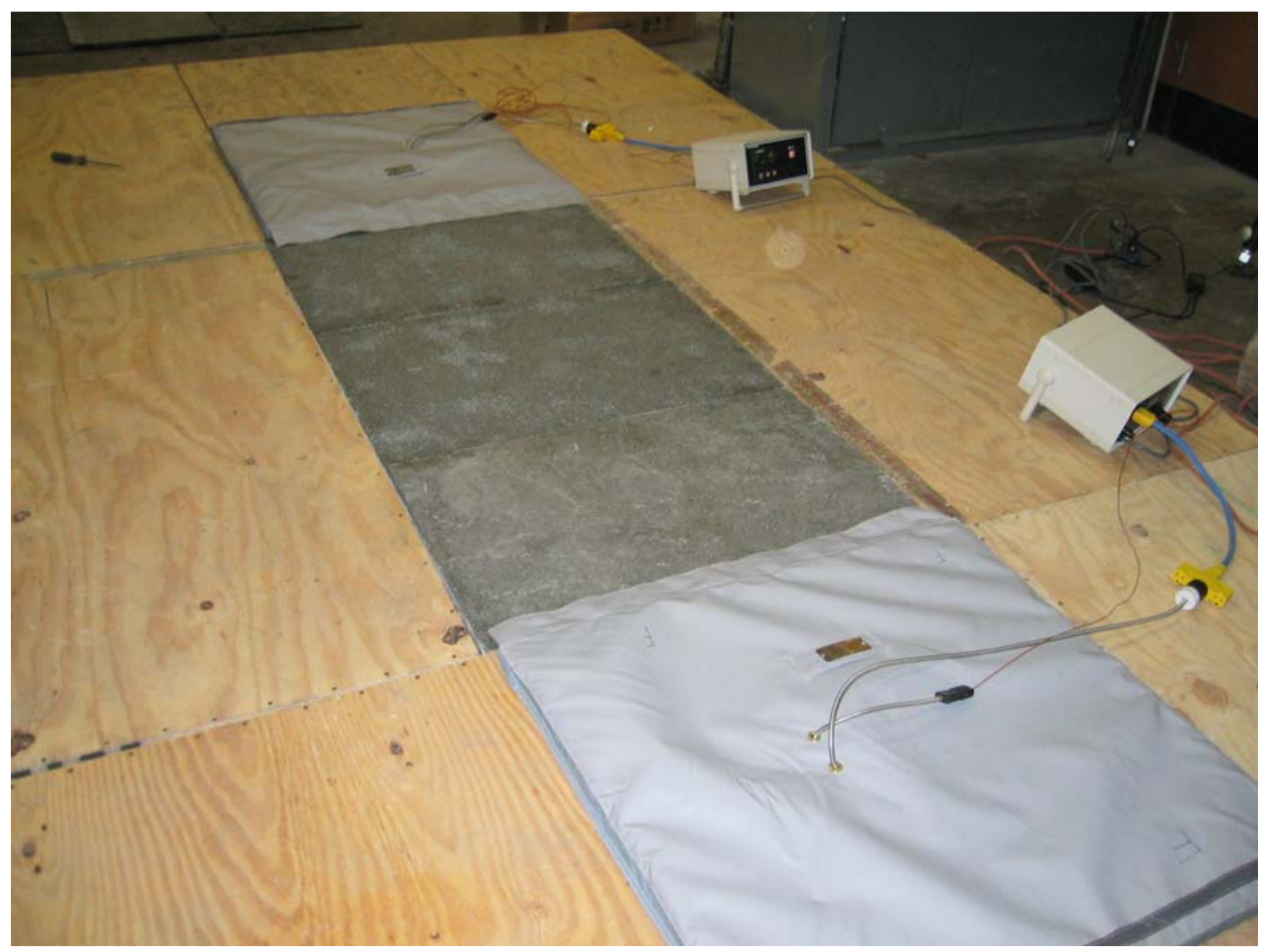

Figure 5.19: The experimental setup for IRT test using heating blanket as active heat source. 


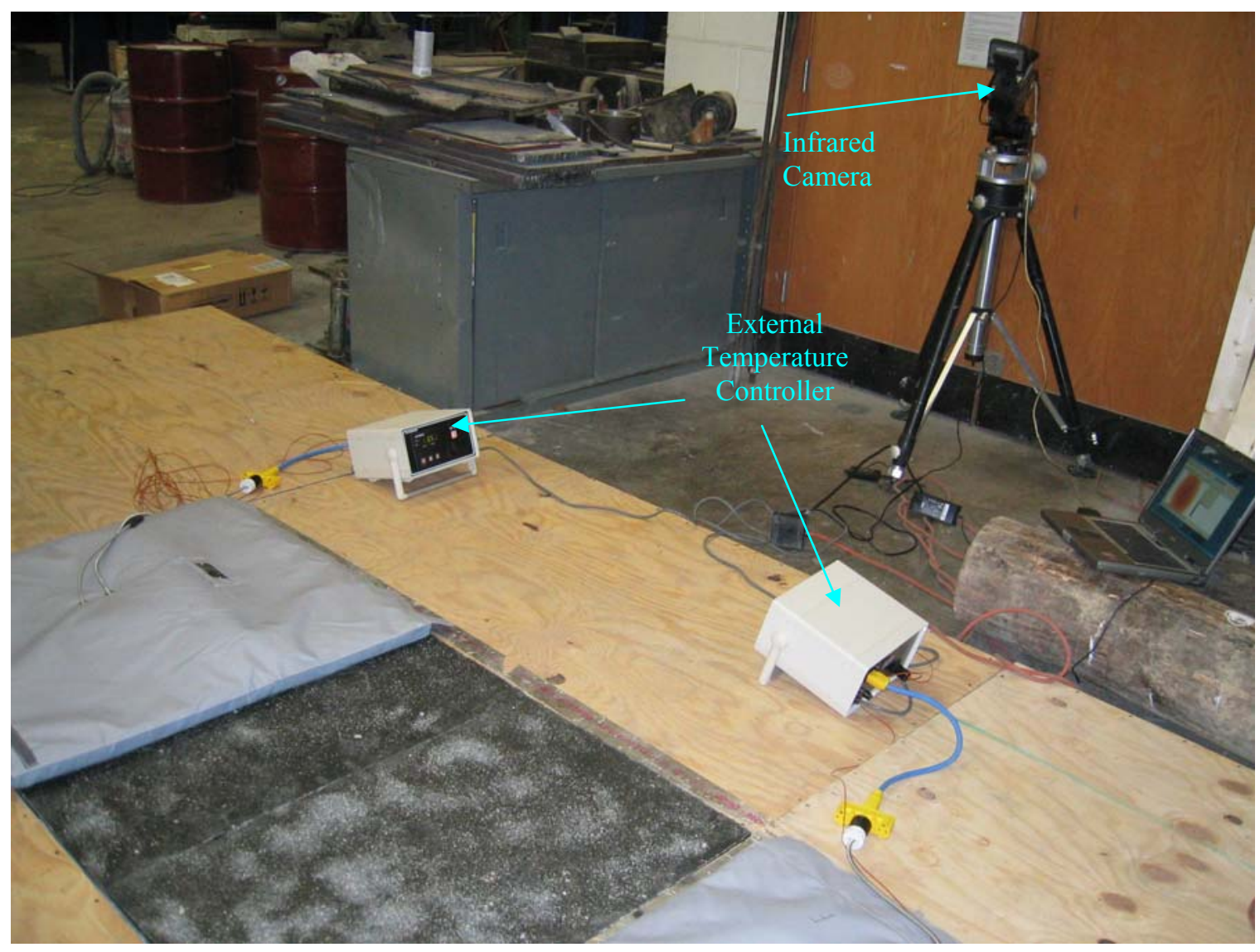

Figure 5.20: The experimental setup for IRT test using heating blanket as active heat source.

The objective of this experiment was to determine the feasibility of IRT using heating blanket as active heat source in defect detection. Therefore, various debond sizes and thicknesses were used with the defect configuration shown in Figures 5.21 and 5.22. Both air-filled and water-filled debonds were used in the experiments. Four minutes of heating at $70^{\circ} \mathrm{F}$ was applied to one $3^{\prime}$ x $3^{\prime}$ portion of the FRP bridge deck. The thermo image acquisition began immediately after the removal of the heating blankets. Three minutes (180 seconds) of thermo images were acquired at one second interval. The process was repeated for another 3' x 3' portion of the FRP bridge deck, and the same process was continued for the other defects configurations. 


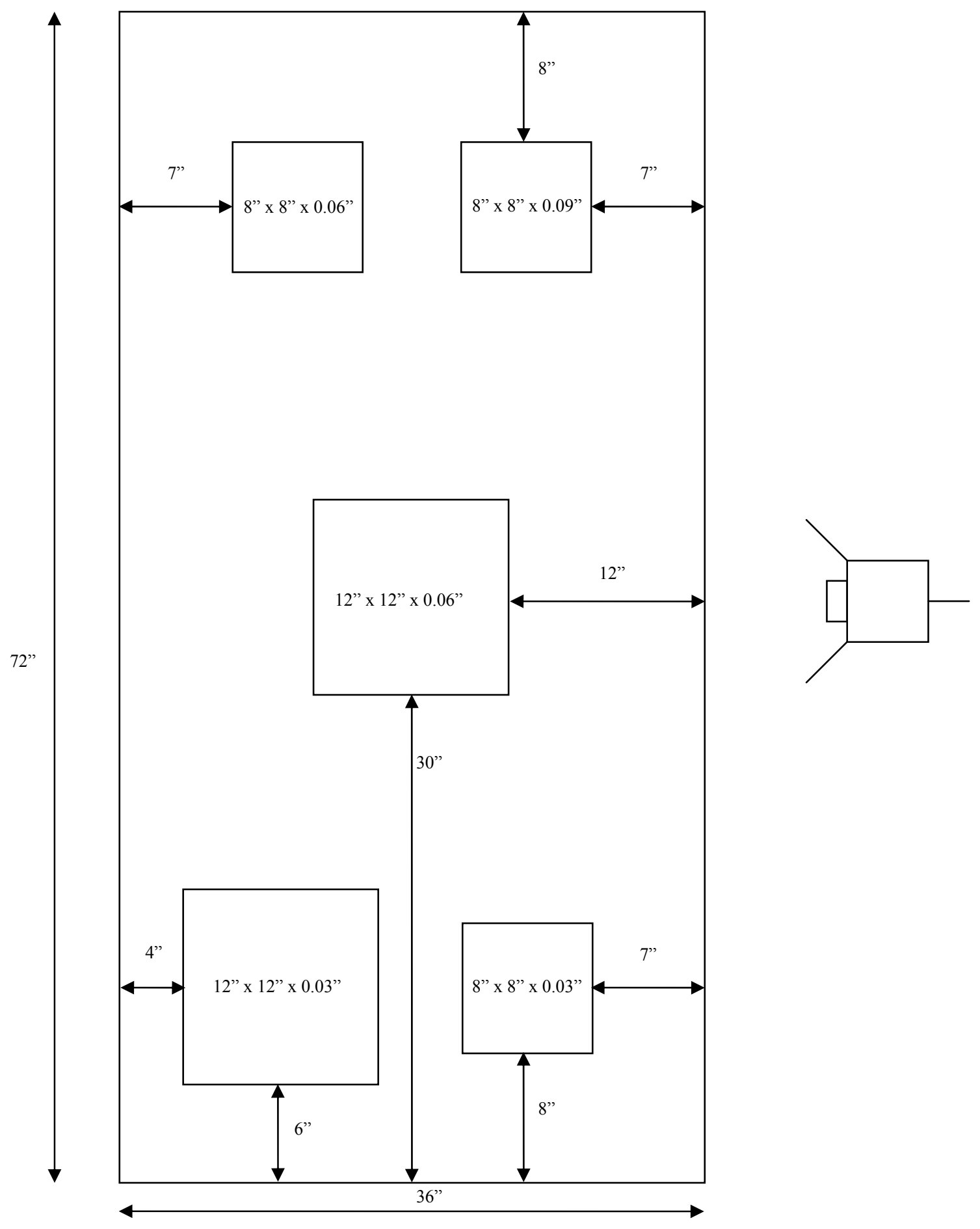

Figure 5.21(a): Defect map for IRT using heating blanket as active heat source. 

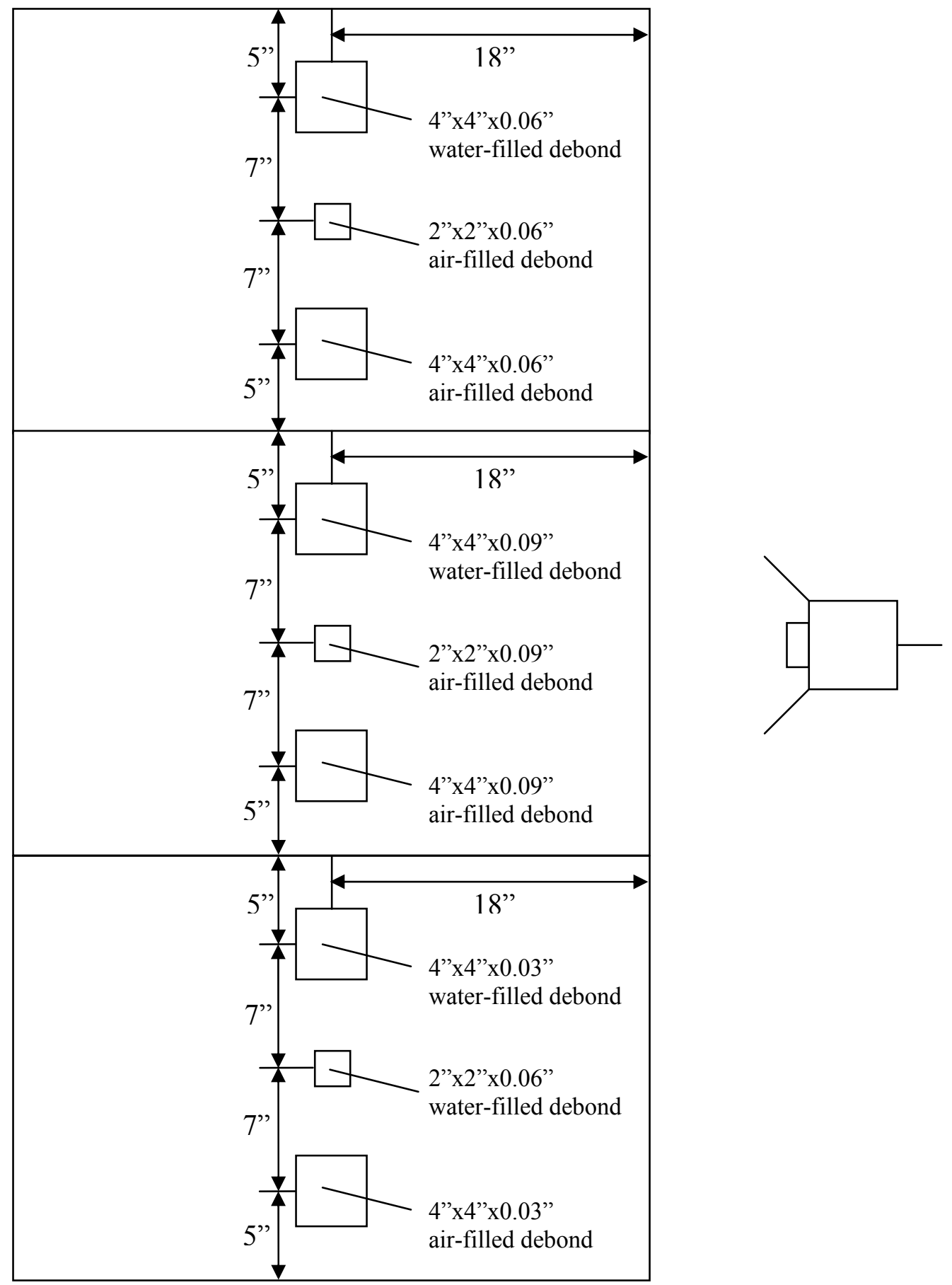

Figure 5.21(b): Defect map for IRT using heating blanket as active heat source. 
As seen in Figure 5.22 and Figure 5.23, all the air-filled defects can be clearly identified. Due to the sheer size of the defects $(8 "$ x 8 " and $12 " \times 12$ ", or $203.2 \mathrm{~mm} \times$ $203.2 \mathrm{~mm}$ and $304.8 \mathrm{~mm} \times 304.8 \mathrm{~mm}$ ), the effect of thickness is not as dominant as before.

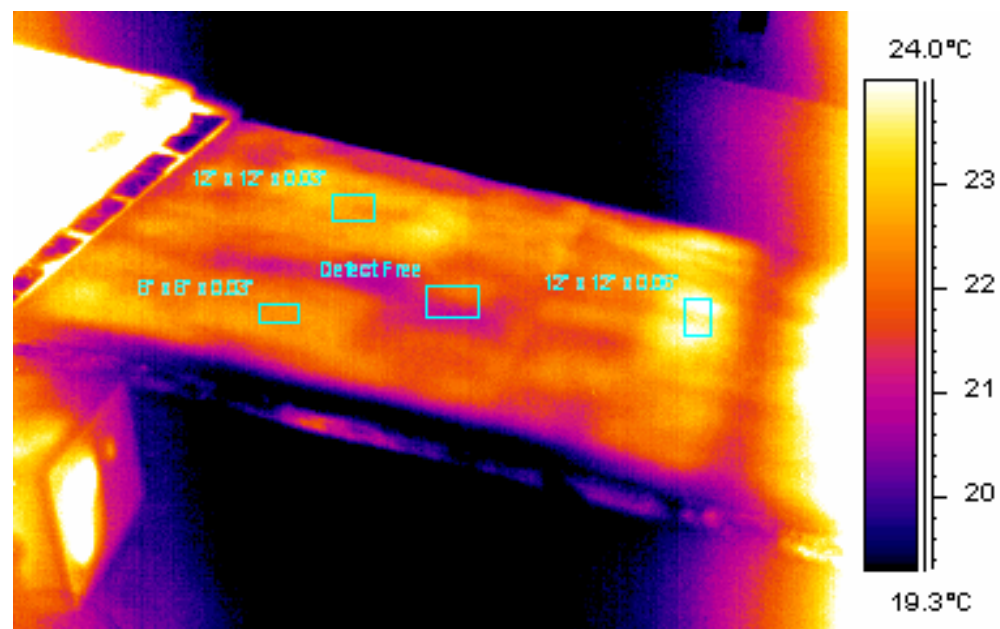

Figure 5.22: Results of IRT on air-filled defects using heating blanket as active heat source.

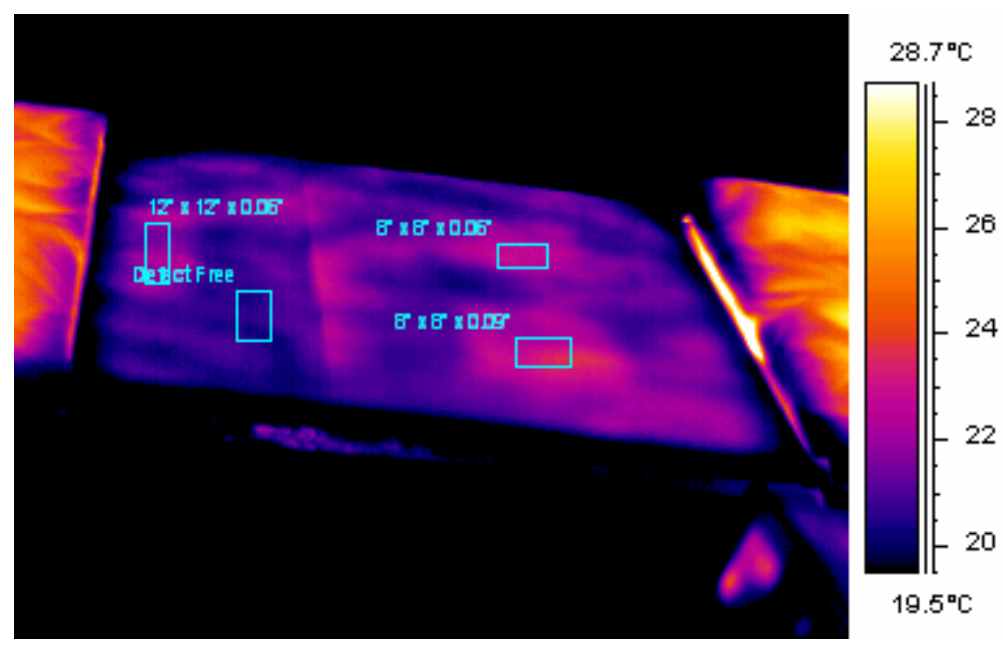

Figure 5.23: Results of IRT on air-filled defects using heating blanket as active heat source.

On the other hand, results for the water-filled defects indicated that thickness is a dominant factor in defect determination using heating blanket as active heat source. As seen in Figure 5.25, the thickest debond of all ( 8 " x 8" x 0.09 " or $203.2 \mathrm{~mm}$ x $203.2 \mathrm{~mm}$ x $2.25 \mathrm{~mm}$ ) can be clearly seen. At the same time, though fuzzy, the 12 " x 12 " $\mathrm{x} 0.03$ " 
$(304.8 \mathrm{~mm} \times 304.8 \mathrm{~mm} \times 0.75 \mathrm{~mm})$ can be detected in Figure 5.24. However, it is important to realize that without prior information, the 12 " x 12 " $\mathrm{x} 0.03$ " $(304.8 \mathrm{~mm} \mathrm{x}$ $304.8 \mathrm{~mm} \times 0.75 \mathrm{~mm}$ ) water-filled debond in Figure 5.24 cannot be accurately identified. The last set of IRT tests were conducted on modules with smaller defects (2" x 2" and 4" x 4" or $50.8 \mathrm{~mm} \times 50.8 \mathrm{~mm}$ and $101.6 \mathrm{~mm} \times 101.6 \mathrm{~mm}$ debonds). As seen in Figures 5.26 and 5.27, the heating blanket did not provide adequate energy to detect any of these smaller defects.

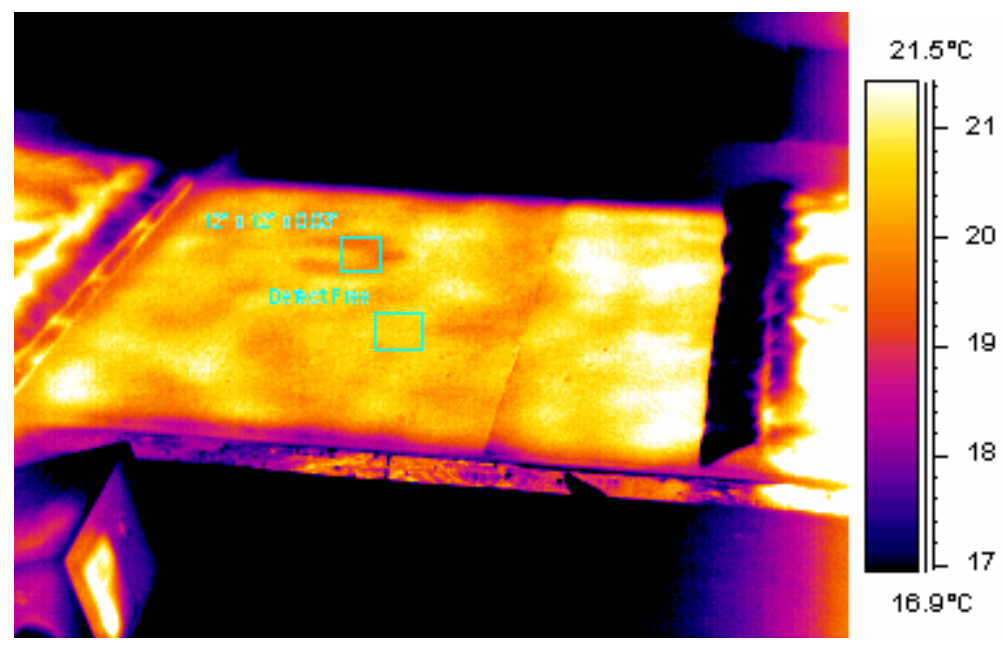

Figure 5.24: Results of IRT on water-filled defects using heating blanket as active heat source.

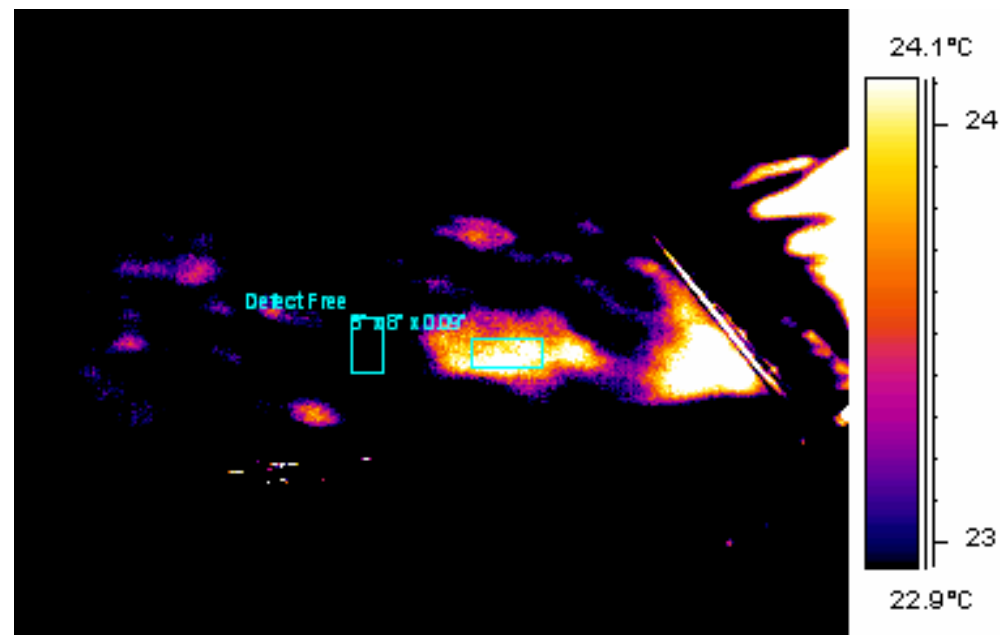

Figure 5.25: Results of IRT on water-filled defects using heating blanket as active heat source. 


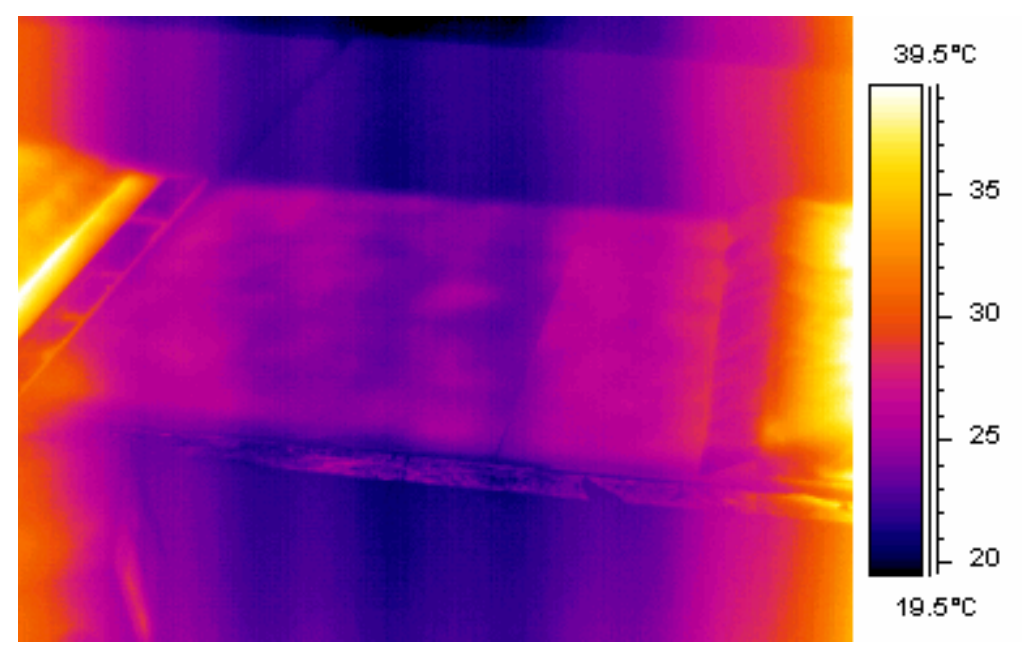

Figure 5.26: Results of IRT on smaller size defects using heating blanket as active heat source.

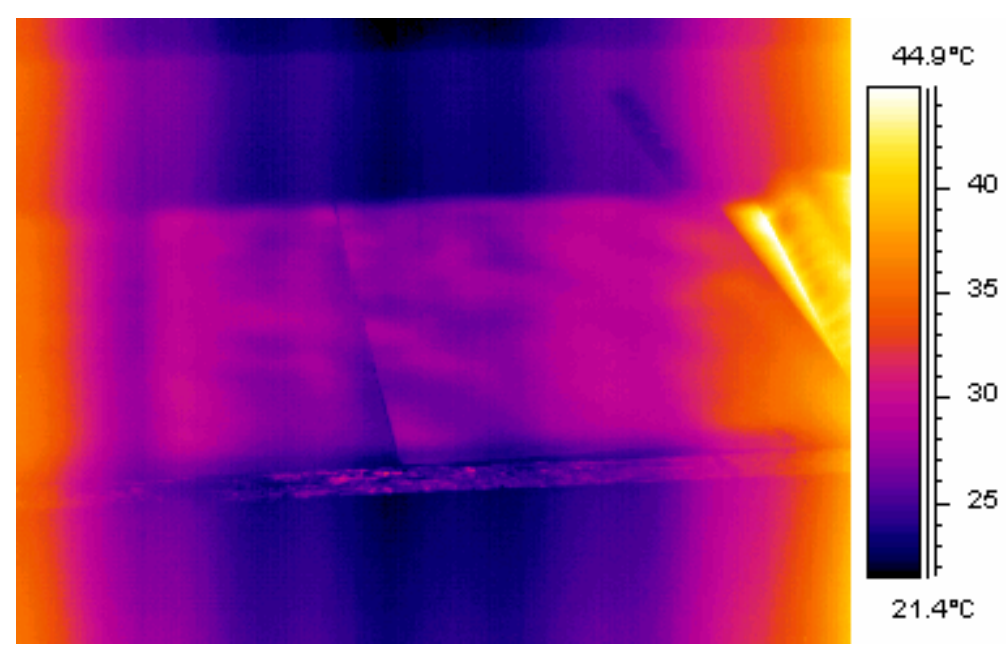

Figure 5.27: Results of IRT on smaller size defects using heating blanket as active heat source.

To further investigate the results obtained so far, the surface temperature of each detectable debond (both air-filled and water-filled) were recorded and plotted using MicrosoftC Excel.

It is clear that the surface temperature over an air-filled debond increases with the volume of the debond. By inspecting Figure 5.28, it can be seen that all the air-filled defects show higher surface temperatures than the defect-free area. Furthermore, in Figure 5.29, the difference between the defective and defect-free area is about 2 degrees 
Celsius for the largest debond (12" x 12" x 0.06 " or $304.8 \mathrm{~mm}$ x $304.8 \mathrm{~mm} \times 1.5 \mathrm{~mm}$ ). From Figure 5.29, it is clear that the effect of thickness is more prominent than the effect of size since the difference between 12 " x 12 " x 0.06 " and 12 " x 12 " x 0.03 " is about 1 degree Celsius while it's about 0.5 degree Celsius for the difference between two different sizes defect of the same thickness. Interestingly, the difference between 8 " x 8 " x $0.09 "$ and $8 "$ x 8 " x 0.06 " air-filled debond is only about 0.25 degree Celsius (Figure 5.30). This indicate that the effect of thickness on surface temperature is tapering down after reaching a thickness of about 0.06 ". In Figure 5.31 the surface temperature of 12 " $\mathrm{x}$ 12 " $\mathrm{x} 0.06$ " is much lower compared to any other defects. This indicates that the heating blanket may not be as effective in its edge region, thus resulting in lower surface temperature near the edge area of the heating blanket.

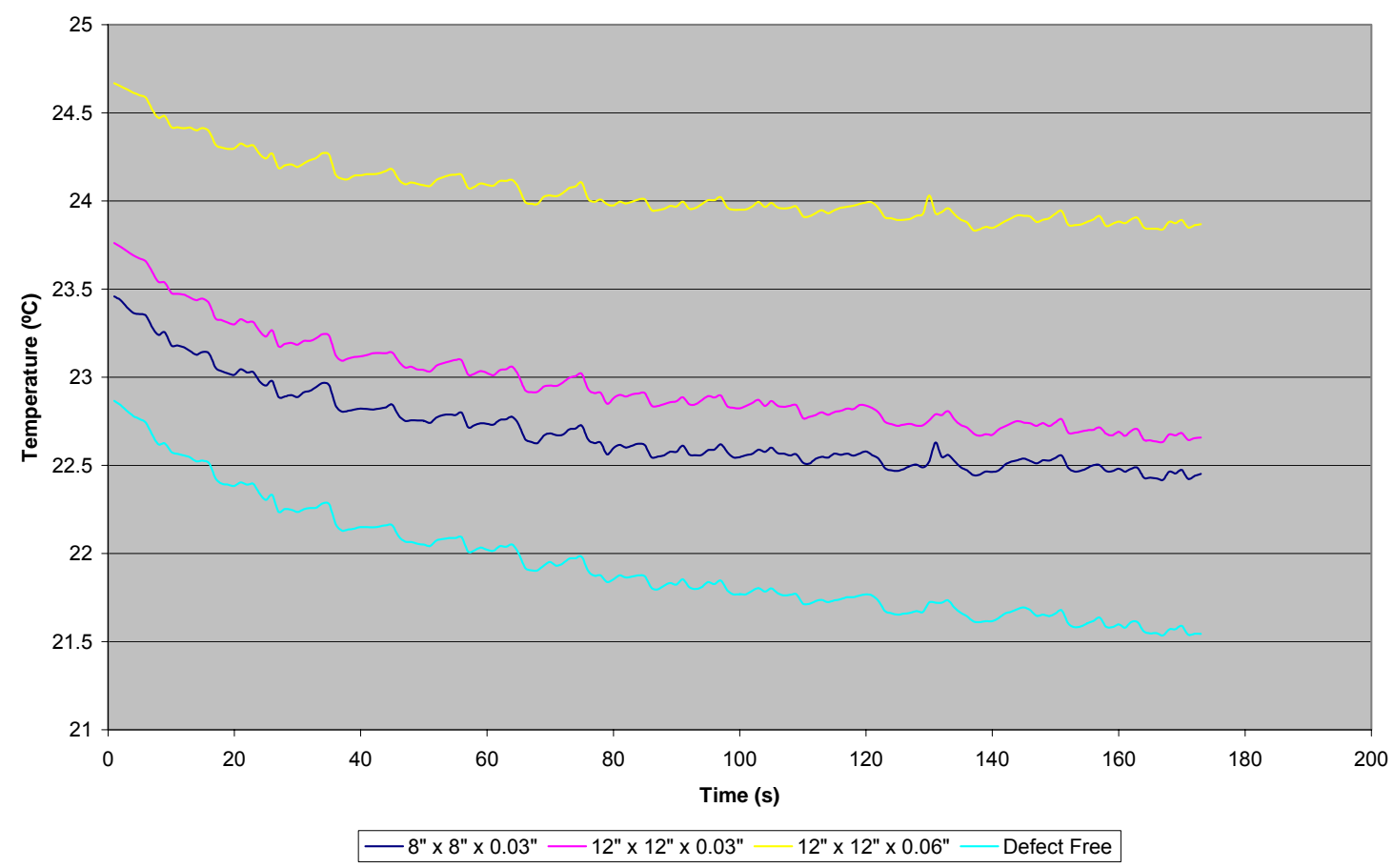

Figure 5.28: Temperature profile of various air-filled debonds. 


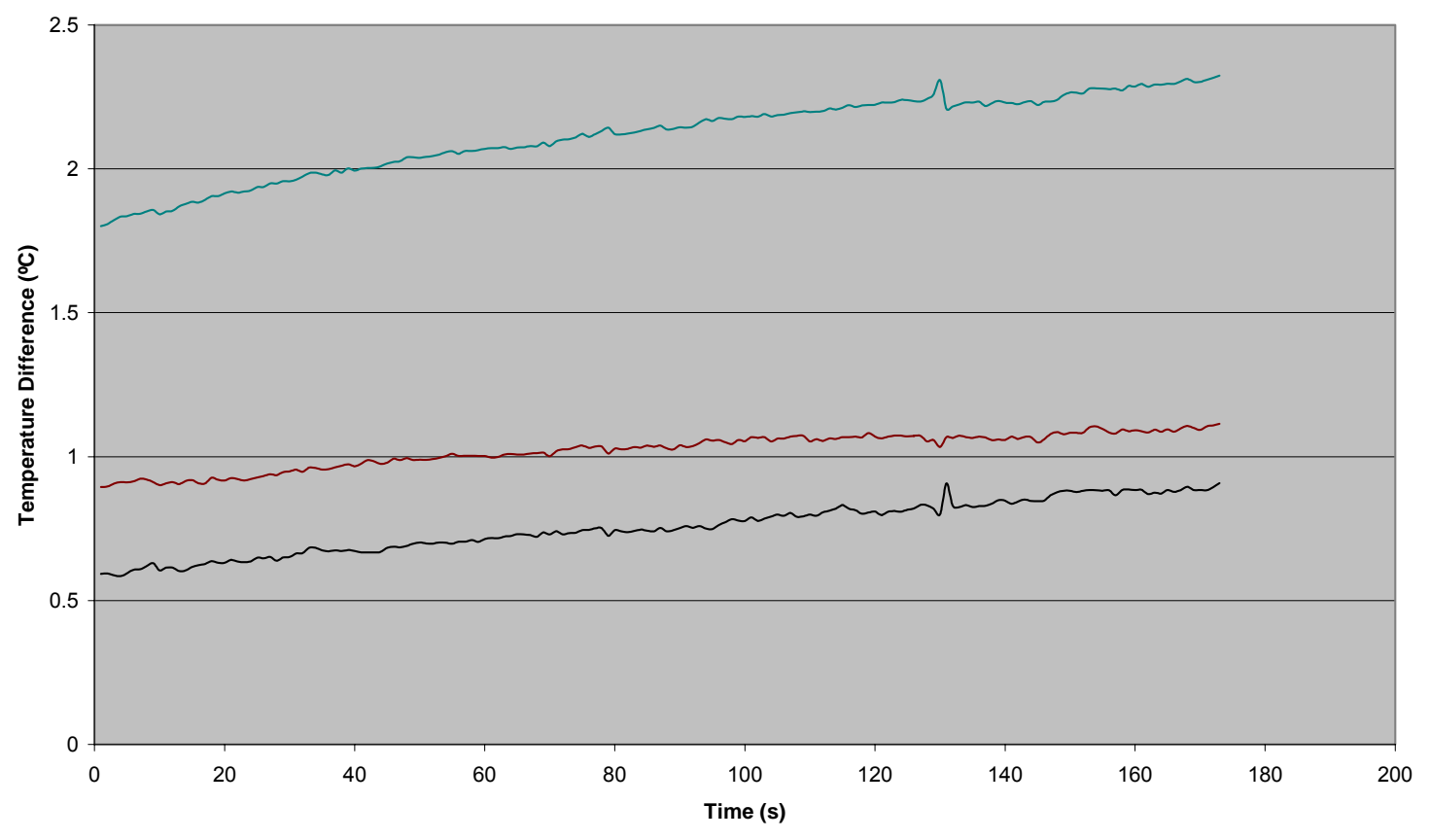

—

Figure 5.29: Temperature profile of defective minus defect-free for various air-filled debonds.

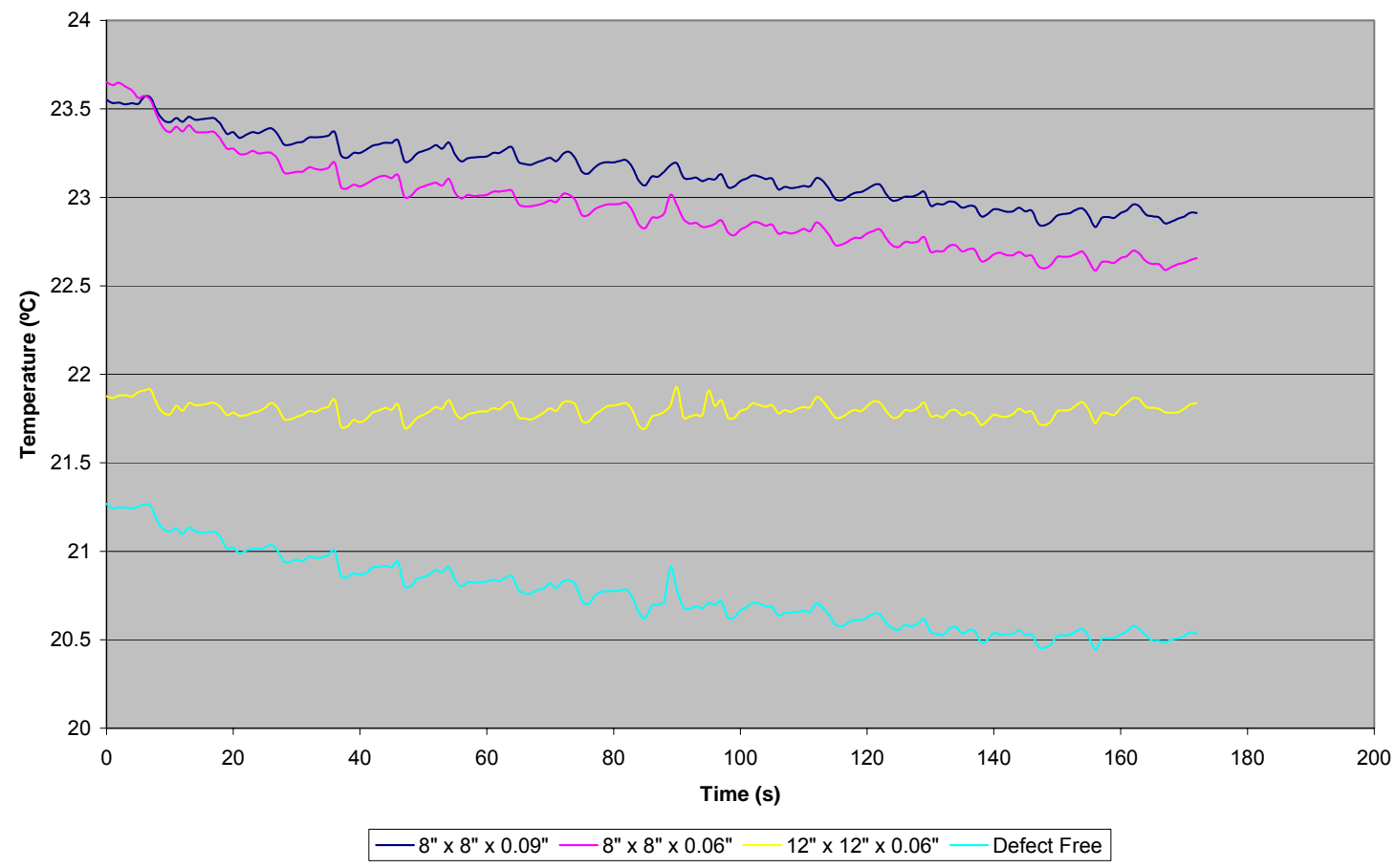

Figure 5.30: Temperature profile of various air-filled debonds. 


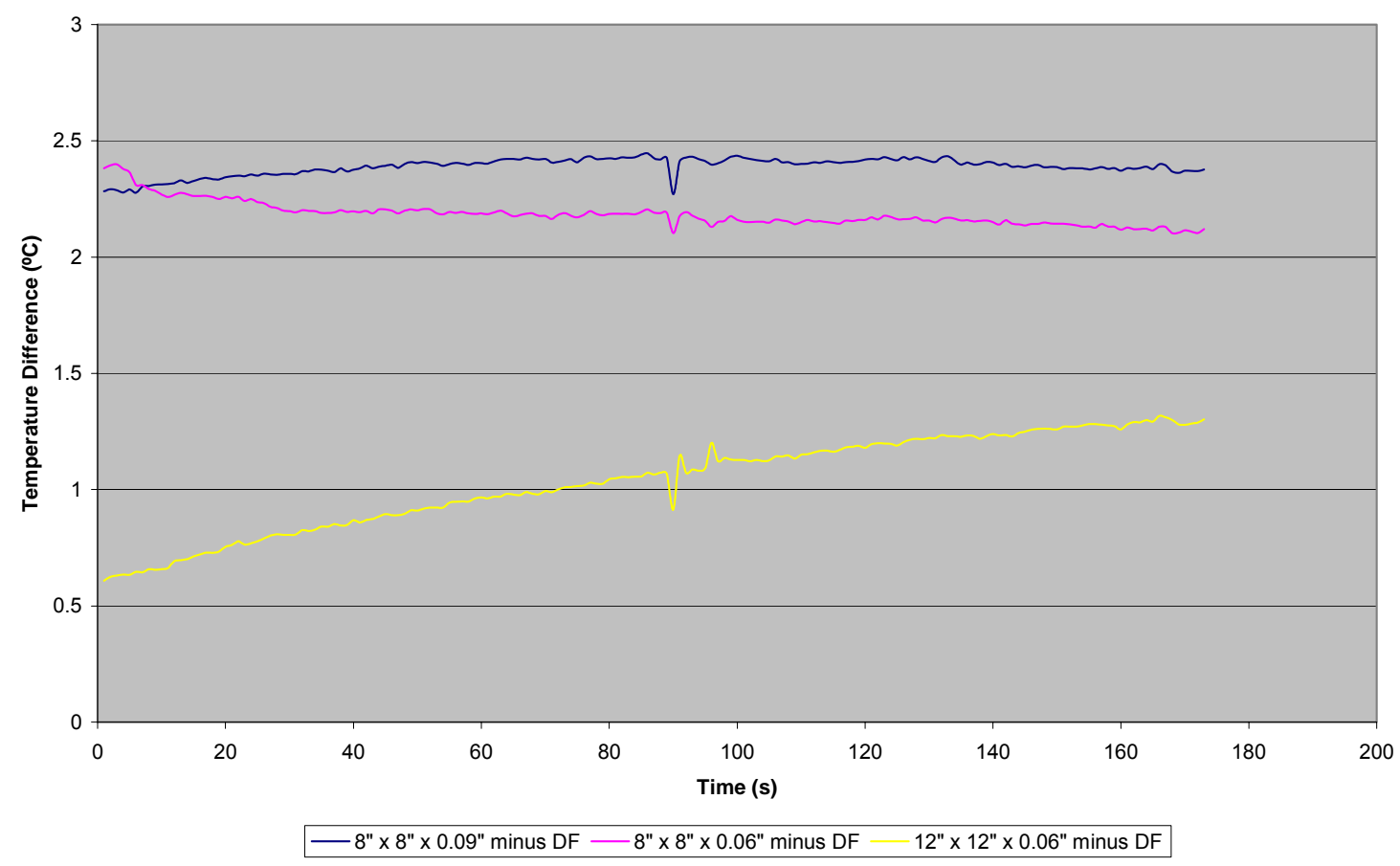

Figure 5.31: Temperature profile of defective minus defect-free for various air-filled debonds.

As indicated before, the sensitivity of IRT in detecting debonds reduced when the debonds are filled with water. As seen in Figure 5.32, the temperature difference dropped to about 0.53 to 0.68 degree Celsius when the 12 " 12 " 12.03 " debonds are filled with water. Likewise, the temperature difference for $8 " \times 8 " \times 0.09 "$ water-filled debond is about 2.1 to 2.2 degree Celsius (Figure 5.33). This indicates a drop of about 0.3 degrees when the debond is filled with water instead of air. It is interesting to note in Figure 5.33 that the temperature difference for the water-filled debonds kept rising for 60 seconds or so before it decreases. This phenomenon is largely due to water's high specific heat value which means it takes longer to get heated compared to FRP. The same phenomenon can also be seen in Figure 5.32, although it is much clearer in Figure 5.33. 


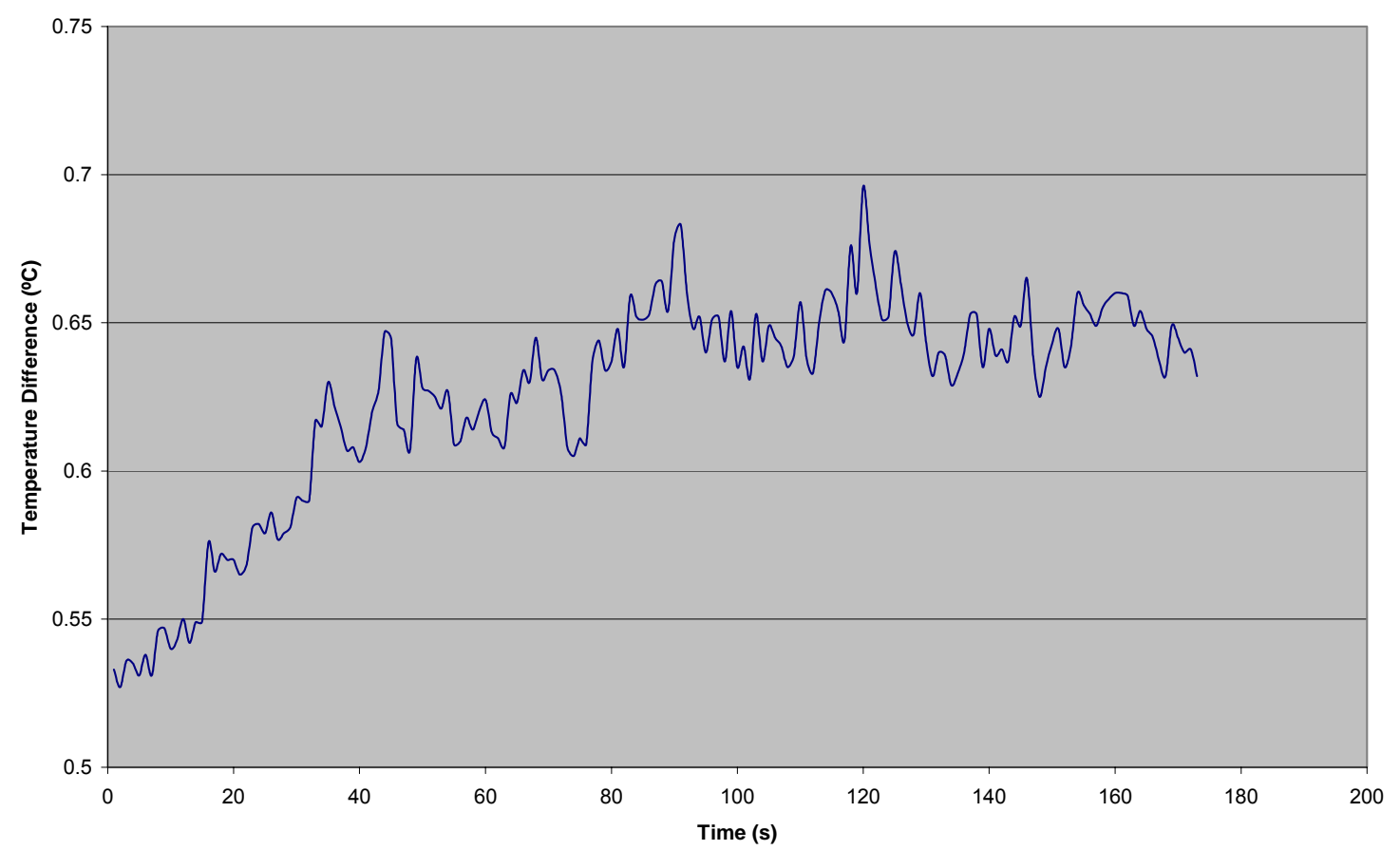

Figure 5.32: Temperature profile of defective minus defect-free for 12 " x 12 " x 0.03 " water-filled debonds.

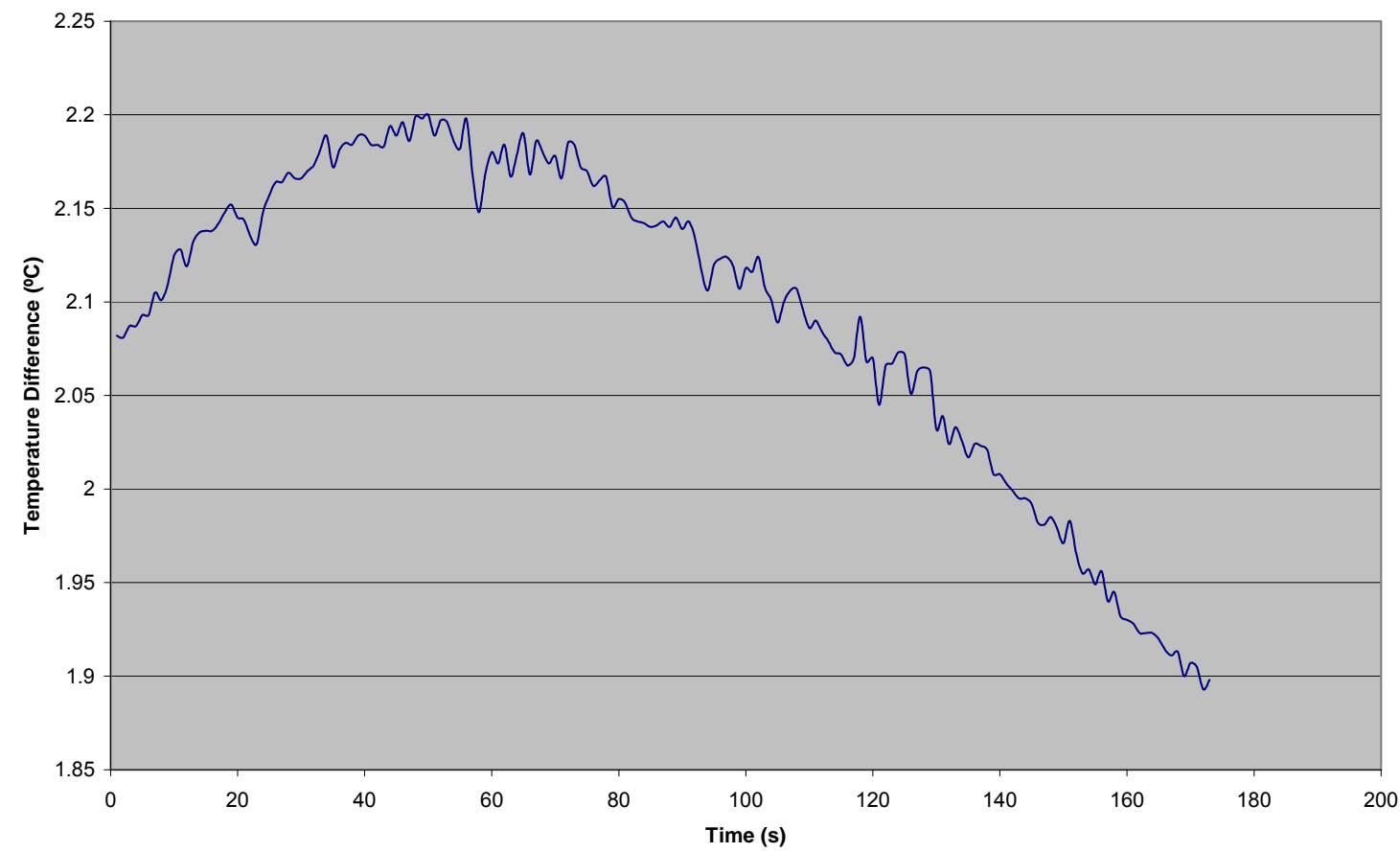

Figure 5.33: Temperature profile of defective minus defect-free for 8 " 8 " x 0.09 " water-filled debonds. 


\subsection{CONCLUSIONS}

In summary, results have shown that while commercially available heaters are capable of finding defects as small as 1 " x 1 " $(25.4 \mathrm{~mm} \times 25.4 \mathrm{~mm})$ in laboratory setting, it is certainly not as good at detecting defects in a larger scale specimen since the smallest air-filled defect that can be detected was 2 " x 2 " x 0.06 " $(50.8 \mathrm{~mm} \times 50.8 \mathrm{~mm} \times 1.5 \mathrm{~mm})$. This is because moving the heater back and forth in larger specimen reduces the heat intensity. This problem can be addressed using multiple heaters, or by testing larger areas in small pieces. Furthermore, use of heaters was not found to be very effective in detecting water-filled defects.

Solar radiation can be a good heating source if used properly. The best times to detect air-filled debonds are between 12 noon to 5 PM. Night time may provide better result for IRT testing if an external heating source is going to be employed since the temperature fluctuation is relatively low at night. A relatively low wind velocity environment is needed to produce good results. The solar radiation tests have shown that 4" x 4" x 0.09 " (101.6mm x 101.6mm x 2.25mm) water-filled debond can be detected on a warm sunny day (for example, during the summer months), while the ability to detect smaller and thinner water-filled debonds need further improvement. Both solar tests have shown that all the 4" x 4" $(101.6 \mathrm{~mm} \times 101.6 \mathrm{~mm})$ air-filled debonds as thin as $0.06 "$ $(1.5 \mathrm{~mm})$ can be detected in warm September weather and colder March days using solar energy as primary heat source. The results also showed that the defect clarity in colder March days was not very good but the warm September day produced good results. The test also showed that delamination within the flanges of FRP decks cannot be detected using only solar radiation. 
The heating blankets proved to be the weakest active heat source explored in this study. It was not capable of detecting any debonds (air-filled and water-filled) smaller than $8 " \times 8$ " in size. Although the heating blankets were sending out $1200 \mathrm{~W} / \mathrm{m}^{2}$ of output (roughly the same amount of energy emitted by the sun on a hot sunny day in June), it did not perform as well as the solar radiation test because the specimens were heated by the sun for a much longer period of time compared to only 4 minutes using the heating blanket. When comparing the heating blanket to the heaters, it is clear that heaters provide a much greater heat intensity ( $\cong 2500 \mathrm{~W} / \mathrm{m}^{2}$ output) for IRT. The only setback regarding the heaters is its inability to apply uniform heat to a large area while the heating blankets provided uniform heating of the specimens. 


\section{Chapter 6}

\section{FINITE ELEMENT MODELING}

Finite element (FE) analysis is widely accepted as a powerful and versatile analytical tool. While finite element could be a powerful tool in analyzing stress-strain and thermal behavior for objects with complex geometry, the goal of this study is to utilize a simple heat transfer finite element model that could be used for studying the effect of subsurface defects on heat transfer through FRP bridge decks and the resulting surface temperature differences.

Before thermal modeling of the FRP bridge deck, some essential information is needed such as, the thermal properties of the FRP layers and wearing surface, the thermal loading conditions (positive or negative heat input), and the boundary conditions for thermal modeling. The thermal properties needed to create an FE model are the specific heat, the emissivity, and the thermal conductivity. Radiation is assumed to be the dominant mode of heat transfer between the wearing surface and the surrounding air while conduction is the dominant mode of heat transfer between the wearing surface, defects, and the FRP layer in this model. A time-step heat flux was used to simulate the active heat source. There were no existing thermal properties available for the FRP used in this study since it is a relatively new material. Although some published tables for thermal properties (Table 3.1) are available, the FRP bridge deck specimens used in this study were tested in order to experimentally determine the specific heat, the thermal conductivity, and the emissivity for these specimens. 


\subsection{THERMOGRAPHIC DEFECT RECOGNITION AND MEASUREMENT THEORY (Shepard et al. 1999)}

The 3-Dimensional diffusion equation of the thermal response of a thick (semiinfinite) opaque solid sample with an insulating subsurface defect, immediately after illumination by spatially uniform light pulse, is described by,

$$
\nabla^{2} T-\frac{1}{\alpha} \frac{\partial T}{\partial t}=0
$$

where, del operator $\nabla^{2}=\frac{\partial^{2}}{\partial x^{2}}+\frac{\partial^{2}}{\partial y^{2}}+\frac{\partial^{2}}{\partial z^{2}}, \alpha$ is the thermal diffusivity and $\mathrm{T}=\mathrm{T}(\mathrm{x}, \mathrm{y}, \mathrm{z})$,

is the temperature of the sample. Using a simple separation of variables, one may treat this equation in such a way that a defect signature based on both spatial and temporal characteristics of the surface profile becomes evident.

In a defect-free sample, at a point far away from the edges, lateral heat flow components cancel and the temperature distribution can be approximated by,

$$
\frac{\partial^{2}}{\partial z^{2}} T-\frac{1}{\alpha} \frac{\partial T}{\partial t}=0
$$

Contrary to that, the presence of an insulating subsurface defect obstructs the flow of heat into the sample and the incident thermal energy is trapped between the defect and the sample surface. The trapped energy flows towards cooler areas surrounding the defect in the lateral direction, which will be the dominant cooling mechanism in the region. The temperature distribution in this case can be approximated by,

$$
\nabla_{x, y}^{2} T-\frac{1}{\alpha} \frac{\partial T}{\partial t}=0
$$



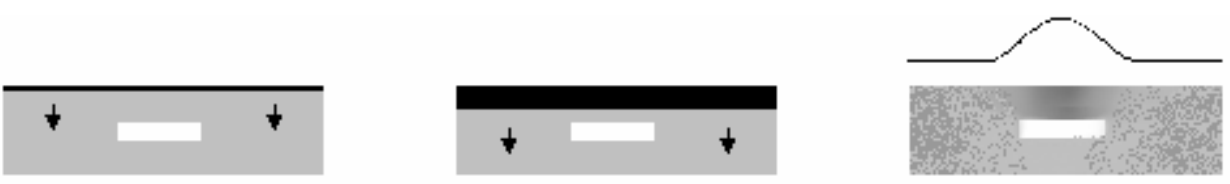

Figure 6.1: Heat flow due to a flash pulse. Initially the pulse causes thin layer of uniform heat on the surface (Left). Heat flows into the sample (Center). When a defect is encountered, the heat is trapped between the defect and the sample surface, giving rise to lateral heat flow above the defect (Shepard et al.

1999).

The analysis of pulsed thermographic data can be separated into two distinct time regimes as a result of the separation of the diffusion equation, a regime before the incident heat reaches the buried defect and that after it reaches the buried defect. The time at which the transition between the regimes occurs depends on the depth of the defect. Until the transition time is reached, the surface above the defect and the rest of the area behave in the same manner. After the elapse of the transition time, the dominant influence on the surface temperature above the defect is the lateral heat flow as per Equation 6.3.

\subsection{THERMAL PROPERTIES}

\subsubsection{Conductivity}

Thermal conductivity means "The material property that describes the rate at which heat flows within a body for a given temperature change." To measure the thermal conductivity, a thermal conductivity testing system (Unitherm model 2022 from ANTER Corp., Pittsburgh, PA) was utilized. Figure 6.2 shows the experimental setup of the test. 


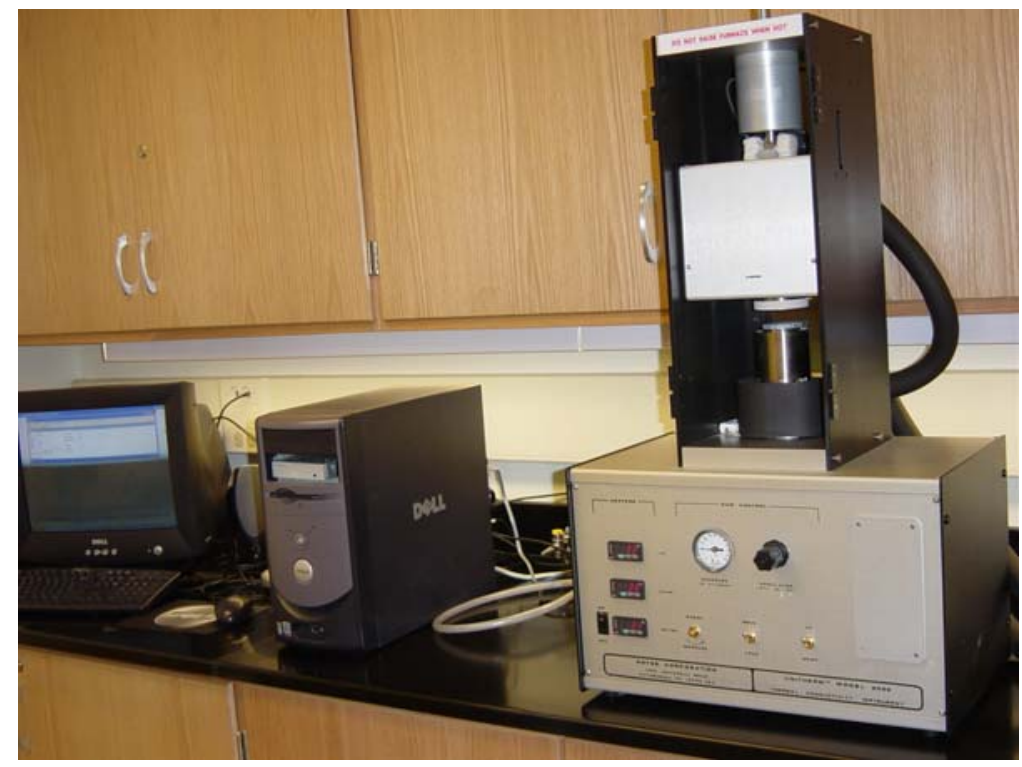

Figure 6.2: Experimental set up of Unitherm 2022.

This unit is supplied with a mid range flux module covering a thermal resistance range from 0.002 to $0.02 \mathrm{~m}^{2} \mathrm{~K} / \mathrm{W}$ and is able to measure the thermal conductivity of materials in the range of 0.1 to $40 \mathrm{~W} / \mathrm{m}-\mathrm{K}$ as per the test standard ASTM E 1530 . Samples were cut in the form of two inch diameter circular discs from the Transpo wearing surface and the FRP deck's top flange for thermal conductivity measurement.

Figure 6.3 shows the schematic arrangement of the system. The system is essentially made up of parts with different functionalities. The heater on top and bottom are used to create steady state heat transfer through the sample. The heat sink at the bottom is to prevent excessive temperature from the system resulting in system failure. A reference calorimeter is placed under the lower plate, which acts as a heat flux transducer. The sample disks are first polished to ensure good thermal contact. Then the prepared sample is placed between two polished surfaces and a pneumatic pressure of $10 \mathrm{psi}$ is applied on the top of the system as indicated in Figure 6.3. The entire system is 
maintained in a thermally insulated glass chamber. The system will check for steady state heat flow through the sample and thermal conductivity is then measured (Mutnuri 2005). Each test takes approximately 3 hours to complete.

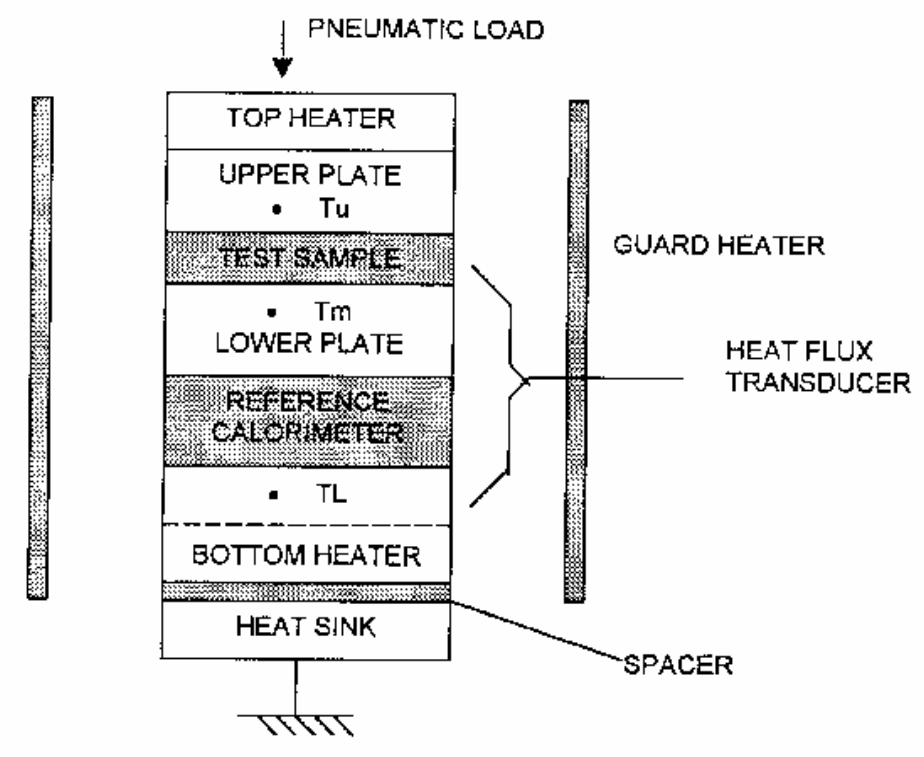

Figure 6.3: Schematic model of Unitherm 2022.

A total of four FRP deck samples and three Transpo wearing surface samples were prepared and tested (Figures 6.4a and 6.4b).

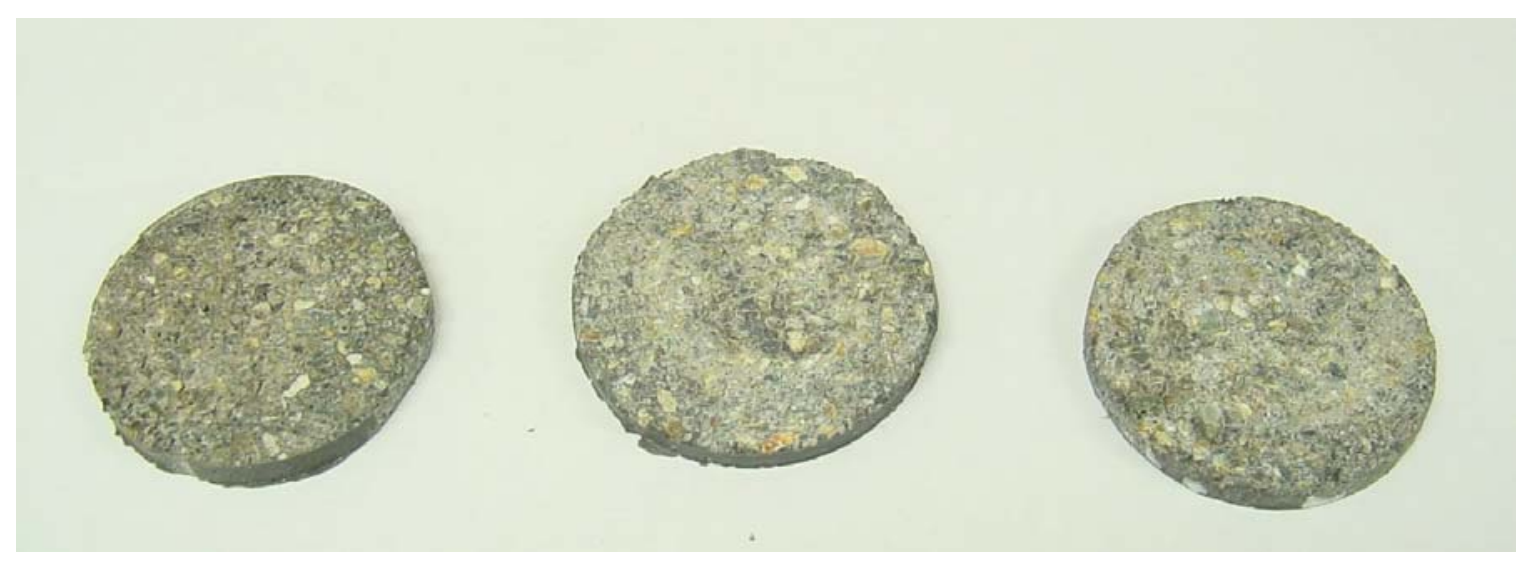

Figure 6.4(a): Circular Transpo wearing surface disc samples. 


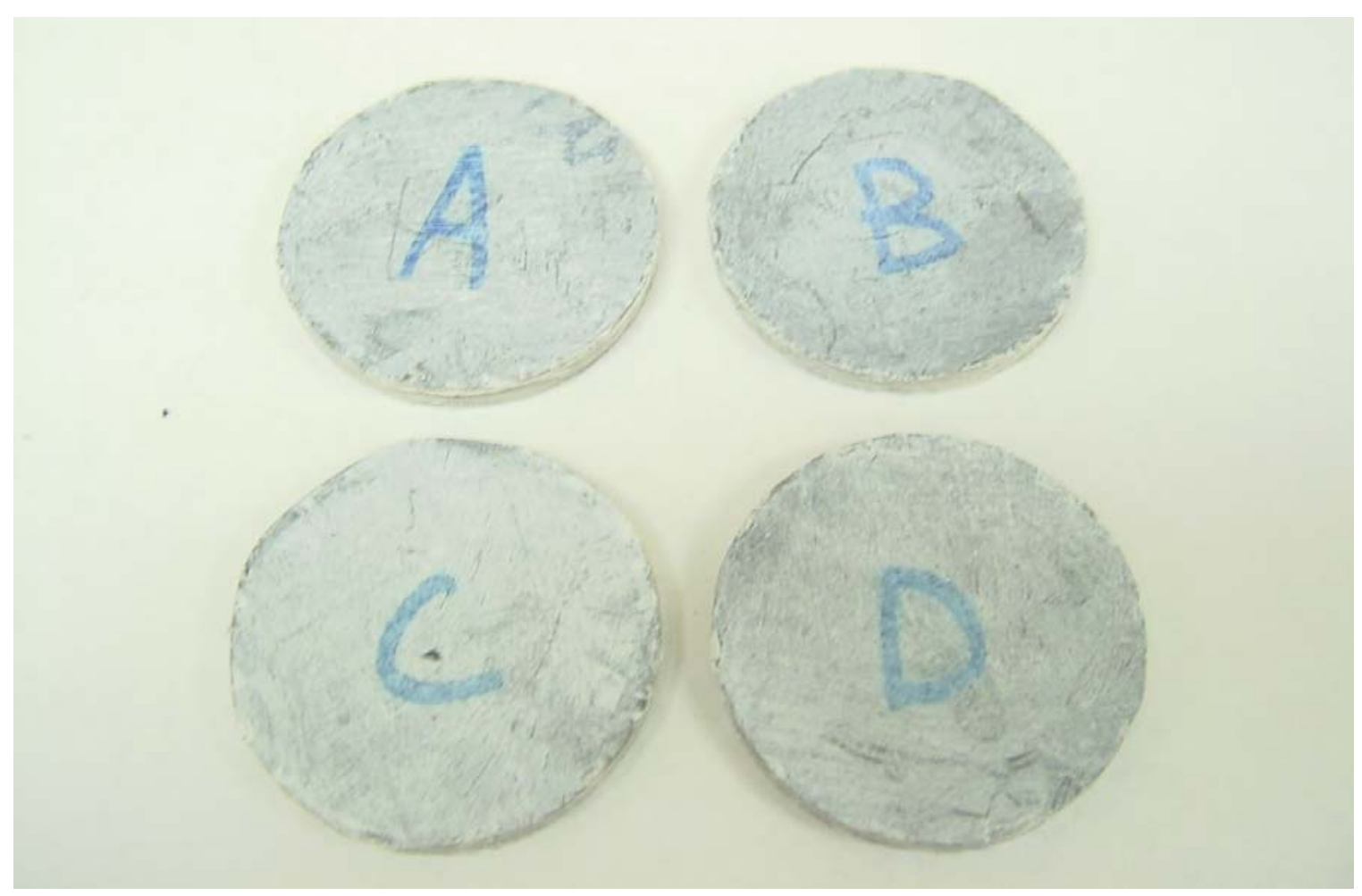

Figure 6.4(b): Circular FRP disc samples.

The thermal conductivity value for the four FRP samples ranged from 0.3 to 0.35 $\mathrm{W} \mathrm{m}{ }^{-1}{ }^{\circ} \mathrm{C}^{-1}$, thus an average of $0.325 \mathrm{~W} \mathrm{~m}^{-1}{ }^{\circ} \mathrm{C}^{-1}$ was used for the $\mathrm{FE}$ modeling. On the other hand, the three Transpo wearing surface samples yielded an average value of 0.63 $\mathrm{W} \mathrm{m}{ }^{-1}{ }^{\circ} \mathrm{C}^{-1}$. 


\subsubsection{Emissivity}

Emissivity refers to the ratio of power radiated by a substance to the power radiated by a blackbody at the same temperature. It is a measure of a material's ability to absorb and radiate energy. Based on the definition, the emissivity value can be, in principal, determined by knowing the apparent (measured) temperature of the interest material and the temperature of a blackbody at the same temperature.

To achieve such goal, a small FRP bridge deck specimen (12" x 24" or 304.8mm $\mathrm{x} 609.6 \mathrm{~mm}$ ) was prepared. Then wearing surface was placed on one half of the specimen, leaving the other half surface of the bridge deck module a FRP surface. Half of the wearing surface and half of the FRP surface were painted black using a special flat-black paint. The flat-black paint produced a near perfect blackbody, which has an emissivity value of 1 .

The bridge deck specimen was then heated using solar energy to ensure uniform heating. The temperature was recorded. The same procedure was then repeated for the other face of the bridge deck specimen.

Through the use of infrared camera, the temperatures of the blackbody region as well as the unaffected region were extracted from the original file. Base on the equation,

$$
\text { Total radiated energy }=\varepsilon(T+273)^{4}
$$

the emissivity value can be calculated with the help of Microsoft Excel. Here $\mathrm{T}$ is measured in ${ }^{\circ} \mathrm{C}$. The region with flat-black paint exhibited a higher temperature. With the known emissivity value calibrated into the infrared camera and the temperature (average 
temperature in this test), the total energy emitted can be calculated. That information was then used to back calculate the actual emissivity value of the material desired.

The emissivity value of the FRP deck's flange was determined to be 0.9 while the emissivity value of the wearing surface was 0.89 .

\subsubsection{Specific Heat}

Specific heat $(\mathrm{Cp})$ is defined as the amount of heat needed to raise the temperature of one unit mass of an object by 1 degree Celsius. As with the other thermal properties, the specific heat value pertaining to the FRP decks used in this experiment is unique, thus required to be determined experimentally.

To determine the specific heat value of the wearing surface as well as the FRP samples, a uniform heating mechanism is needed to increase the temperature to a known value. A commercially available digital deep fryer was chosen for this task (Figure 6.5).

The sample was first weighed using a digital scale (Figure 6.6). It was then heated in vegetable oil, which has a specific heat value of $1670 \mathrm{~J} / \mathrm{kg}-\mathrm{C}$, to a temperature of 160 ${ }^{\circ} \mathrm{F}\left(71.1^{\circ} \mathrm{C}\right)$. Upon reaching the equilibrium temperature, the sample was then moved to a beaker with a known volume of vegetable oil at room temperature. The temperature of the vegetable oil was recorded prior to submerging the sample. The temperature of the vegetable oil in the beaker was then carefully monitored, and the maximum temperature was recorded. 


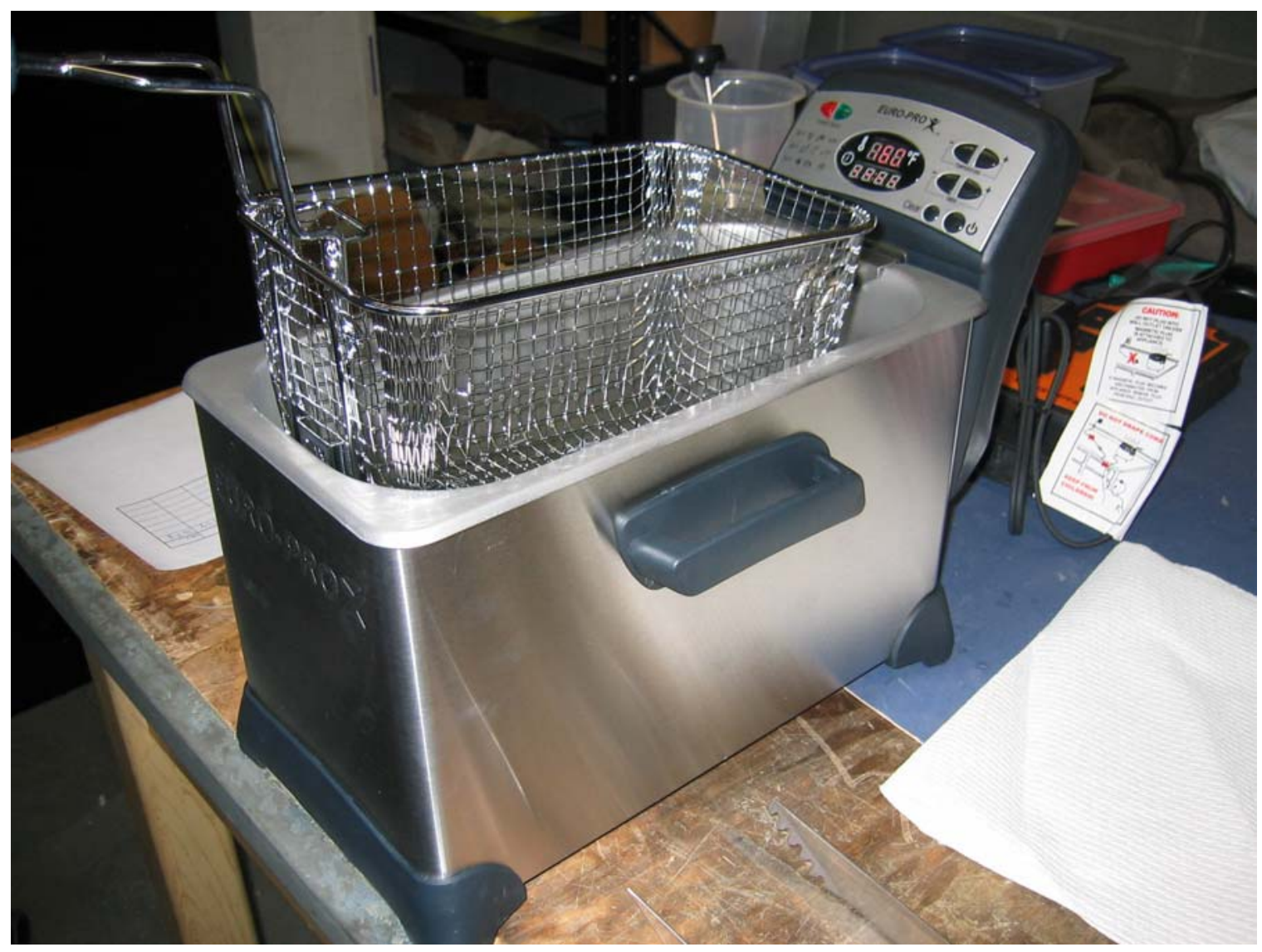

Figure 6.5: The digital deep fryer.

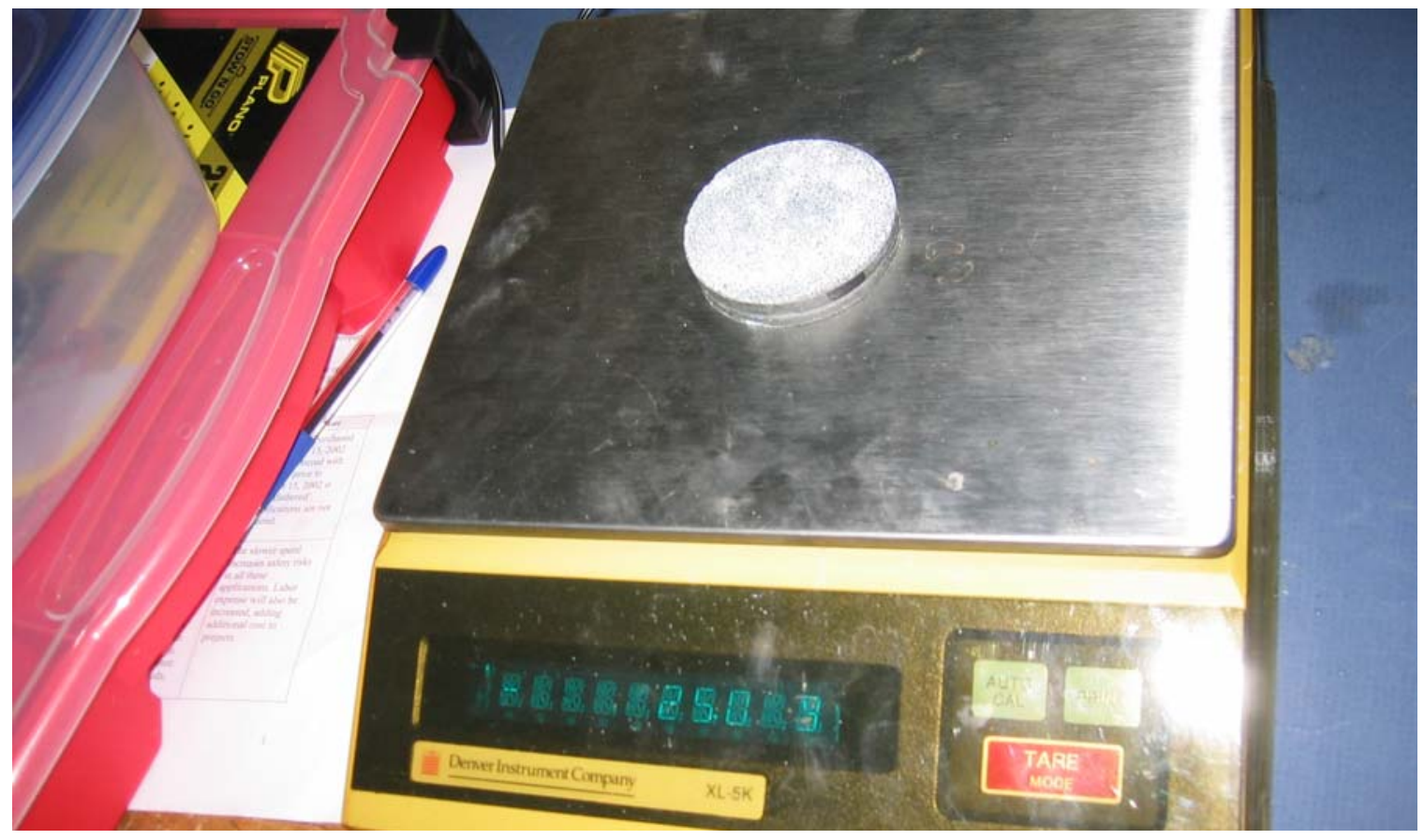

Figure 6.6: Weighing of the sample using a digital scale. 
By knowing three sets of temperature as well as the specific heat value of the vegetable oil and the weight of both vegetable oil and sample, the specific heat value of the sample was easily calculated by using the following equation:

$$
\text { (weight of sample) }\left(\Delta \mathrm{t}_{\text {sample }}\right)\left(\mathrm{Cp}_{\text {sample }}\right)=(\text { weight of oil })\left(\Delta \mathrm{t}_{\text {oil }}\right)\left(\mathrm{Cp}_{\text {oil }}\right)
$$

Table 6.1: The recorded data sheet for the specific heat value test

\begin{tabular}{|c|c|c|c|c|}
\hline & FRP $1(\mathrm{~A})$ & FRP $2(B)$ & FRP $3(\mathrm{C})$ & FRP $4(\mathrm{D})$ \\
\hline Weight of Sample disc, $(\mathrm{g})$ & 21.8 & 18.6 & 18.8 & 21 \\
\hline Temperature of Fryer Oil, (F) & 130 & 130 & 130 & 130 \\
\hline Weight of Vegetable oil, (g) & 77.3 & 77.9 & 77.9 & 77.6 \\
\hline Initial Temperature of Veg. oil, (F) & 78 & 78.7 & 78 & 78.2 \\
\hline Final Temperature of Veg. oil, (F) & 85.8 & 85.5 & 84.8 & 85.9 \\
\hline$\Delta \mathrm{t}_{\mathrm{FRP}}$ & 44.2 & 44.5 & 45.2 & 44.1 \\
\hline$\underline{\Delta \mathrm{t}_{\mathrm{oil}}}$ & 7.8 & 6.8 & 6.8 & 7.7 \\
\hline \multirow[t]{2}{*}{ Specific Heat Value, $(\mathrm{J} / \mathrm{kg}-\mathrm{C})$} & 1045.0 & 1068.8 & 1041.0 & 1077.5 \\
\hline & WS 1 & WS 2 & & \\
\hline Weight of Sample disc, $(\mathrm{g})$ & 42.9 & 39.6 & & \\
\hline Temperature of Fryer Oil, $(\mathrm{F})$ & 130 & 130 & & \\
\hline Weight of Vegetable oil, (g) & 77.4 & 77.6 & & \\
\hline Initial Temperature of Veg. oil, $(\mathrm{F})$ & 77.5 & 81.1 & & \\
\hline Final Temperature of Veg. oil, (F) & 88.4 & 90.3 & & \\
\hline$\Delta \mathrm{t}_{\mathrm{FRP}}$ & 41.6 & 39.7 & & \\
\hline$\Delta \mathrm{t}_{\text {oil }}$ & 10.9 & 9.2 & & \\
\hline Specific Heat Value, $(\mathrm{J} / \mathrm{kg}-\mathrm{C})$ & 789.5 & 758.4 & & \\
\hline \multicolumn{5}{|c|}{$\Delta \mathrm{t}_{\mathrm{FRP}}=($ Temperature of Fryer Oil $)-($ Final Temperature of Veg. oil $)$} \\
\hline \multicolumn{5}{|c|}{$\Delta \mathrm{t}_{\mathrm{oil}}=($ Final Temperature of Veg. oil $)-($ Initial Temperature of Veg. oil $)$} \\
\hline $\begin{array}{l}\text { FRP }=\text { Fiber Reinforced Polym } \\
\text { WS }=\text { Wearing Surface Disc }\end{array}$ & Disc & & & \\
\hline
\end{tabular}


Besides thermal conductivity, emissivity, and specific heat values, the density of the materials are also required. Table 6.2 and Table 6.3 show the density calculation for FRP and wearing surface (WS) material.

Table 6.2 Density table of round 2" diameter FRP and WS specimen

\begin{tabular}{|c|c|c|c|c|}
\hline Material & FRP A & FRP B & FRP C & FRP D \\
\hline diameter, $\mathrm{d}(\mathrm{mm})$ & 50.5 & 50.65 & 50.9 & 50.95 \\
\hline diameter, d (m) & 0.0505 & 0.05065 & 0.0509 & 0.05095 \\
\hline thickness, $\mathrm{t}(\mathrm{mm})$ & 5.37 & 4.77 & 4.93 & 5.16 \\
\hline thickness, t (m) & 0.00537 & 0.00477 & 0.00493 & 0.00516 \\
\hline weight, $\mathrm{m}(\mathrm{kg})$ & 0.0218 & 0.0186 & 0.0188 & 0.021 \\
\hline Density $\left(\mathrm{kg} / \mathrm{m}^{3}\right)$ & 2025.978 & 1934.51 & 1873.315 & 1995.338 \\
\hline Average & $\mathrm{FRP}=$ & 1957.285 & & \\
\hline Material & WS* A & WS* B & WS* C & \\
\hline diameter, $\mathrm{d}(\mathrm{mm})$ & 47.4 & 48.4 & 48.4 & \\
\hline diameter, d (m) & 0.0474 & 0.0484 & 0.0484 & \\
\hline thickness, $\mathrm{t}(\mathrm{mm})$ & 5.25 & 5.7 & 5.9 & \\
\hline thickness, t (m) & 0.00525 & 0.0057 & 0.0059 & \\
\hline weight, $\mathrm{m}(\mathrm{kg})$ & 0.0201 & 0.021 & 0.0235 & \\
\hline Density $\left(\mathrm{kg} / \mathrm{m}^{3}\right)$ & 2168.78 & 2001.654 & 2164.016 & \\
\hline Average & $\mathrm{WS}=$ & 2111.483 & & \\
\hline
\end{tabular}

* Wearing Surface Coupon Sample

Table 6.3 Density table of square FRP specimen

\begin{tabular}{|l|l|l|l|l|}
\hline Square Specimen & A (FRP) & B (FRP) & C (FRP) & \\
\hline Volume, Calculated $\left(\mathrm{m}^{3}\right)$ & 0.00010731 & 0.00010682 & 0.00011035 & \\
\hline Weight $(\mathrm{kg})$ & 0.2051 & 0.2107 & 0.2143 & Average \\
\hline Density, Calculated $\left(\mathrm{kg} / \mathrm{m}^{3}\right)$ & 1911.3 & 1972.5 & 1942 & 1941.33 \\
\hline
\end{tabular}

Sample Calculation:

Round Spec. $\rightarrow$ Volume $=\left[\left(\pi \mathrm{d}^{2}\right) / 4\right]^{*} \mathrm{t}=\left(\frac{22}{7} \frac{(0.0505 \mathrm{~m})^{2}}{4}\right) x 0.00537 \mathrm{~m}=0.0000108 \mathrm{~m}^{3}$

Square Spec. $\rightarrow$ Volume $=\mathrm{b} * \mathrm{~h} * \mathrm{t}=0.1002 \mathrm{~m} \times 0.1022 \mathrm{~m} \times 0.0105 \mathrm{~m}=0.00010731 \mathrm{~m}^{3}$ Density $=$ Weight $/$ Volume $=0.2051 \mathrm{~kg} / 0.00010731 \mathrm{~m}^{3}=1911.3 \mathrm{~kg} / \mathrm{m}^{3}$ 
Table 6.4 shows the thermal properties needed to create the FE model for this study.

Table 6.4 Material properties of GFRP, wearing surface, and water

\begin{tabular}{|l|l|l|l|}
\hline Material & GFRP & Wearing Surface & \multicolumn{1}{c|}{ Water } \\
\hline Mass Density, $\rho\left(\mathrm{kg} \mathrm{m}^{-3}\right)$ & $1950.5^{*}$ & 2111.5 & 1000 \\
\hline Conductivity, $\mathbf{K}\left(\mathrm{W} \mathrm{m}^{-1}{ }^{\circ} \mathrm{C}^{-1}\right)$ & 0.325 & 0.63 & 0.6 \\
\hline Specific Heat, $\mathbf{C p}\left(\mathrm{J} \mathrm{kg}^{-1} \mathrm{C}^{-1}\right)$ & 1058 & 774 & 4180 \\
\hline Emissivity & 0.9 & 0.89 & $0.95^{* *}$ \\
\hline
\end{tabular}

* Average of seven samples

** Obtained from http://www.engineeringtoolbox.com/36_447.html

It is interesting to compare the experimentally obtained values shown in Table 6.4 to that of the published values from Table 3.1. The specific heat value for a glass fiber reinforced polymer (GFRP) is $1200 \mathrm{~J} \mathrm{~kg}^{-1}{ }^{\circ} \mathrm{C}^{-1}$ while the experimentally determined value is at $1058 \mathrm{~J} \mathrm{~kg}^{-1}{ }^{\circ} \mathrm{C}^{-1}$. Furthermore, the experimentally determined thermal conductivity is within $0.025 \mathrm{~W} \mathrm{~m}^{-1}{ }^{\circ} \mathrm{C}^{-1}$ of the value shown in Table 3.1. Likewise, the mass density is only $50.5 \mathrm{~kg} \mathrm{~m}^{-3}$ higher than the value in Table 3.1 .

\subsection{FE MODEL}

The FE model in this study has a dimension of $0.3 \mathrm{~m} \times 0.6 \mathrm{~m}$. SI units were used throughout the modeling process due to the fact that all thermal properties were obtained in SI units. The FE model has two layers of material, with wearing surface as the top layer, and the FRP deck's top flange as the bottom layer. For simplicity purposes, each layer of material contains only one layer of mesh in the thickness direction. Further mesh refinement can be done for future study. The thermal properties, which are predominantly affected by the properties of resin instead of the glass fibers, were assumed to be the same in all 3 directions. The FE model considered 3-dimensional heat transfer. 
Two planar surfaces were defined to represent each of the two materials. The planar surfaces were then extruded into solid element and merged at the boundary. To simulate air-filled debond, a void was extruded from the wearing surface layer, thus creating an air gap. To simulate water-filled debond, water's property was assigned to the void that was extruded from the wearing surface layer.

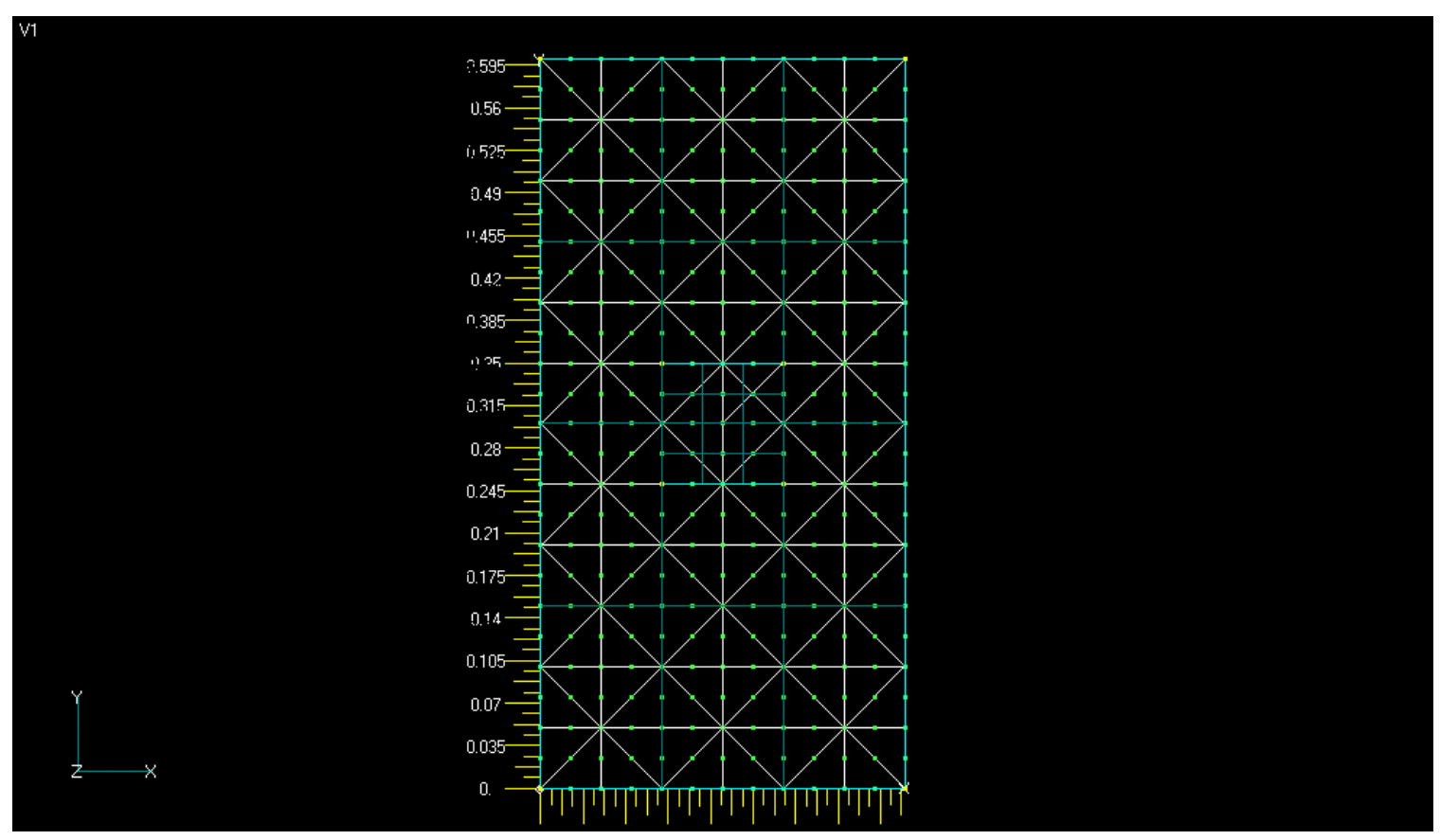

Figure 6.7: Triangular solid mesh of the FE model.

The mesh chosen for this study is a solid triangular mesh (Figure 6.7). Figure 6.8 shows the mesh for the wearing surface layer with a debond embedded in the layer.

Two mesh sizes were utilized: $0.05 \mathrm{~m}$ and $0.025 \mathrm{~m}$. Several cases of $0.05 \mathrm{~m}$ mesh were generated for comparison purposes. The majority of the generated FE models in this study are of $0.025 \mathrm{~m}$ mesh size. The thickness of the element for wearing surface and flange of FRP deck was typically $0.0095 \mathrm{~m}$, that is, each layer (FRP and wearing surface) was one element thick. The void (debond) thickness was of the order of $0.0025 \mathrm{~m}$. This 
means the aspect ratio (element size:thickness) was 10:1 for the smaller mesh size and 20:1 for the bigger mesh size in case of debonds.

A heat flux of 1000 watts $/ \mathrm{m}^{2}$ was used to simulate the active heat source. This heat intensity is similar to that provided by the heating blanket discussed in Chapter 5. To properly monitor the heat transfer, a time-step function was used in conjunction with the heat flux. The heat flux was applied for 300 seconds (5 minutes) and was removed immediately after that. Only radiation is used for heat transfer between the material and the air. The entire model was applied with an initial body temperature of $20{ }^{\circ} \mathrm{C}$ and the ambient air temperature (boundary conditions) were also set to be $20{ }^{\circ} \mathrm{C}$. Once the model was analyzed, readings were noted at 350 seconds, 500 seconds, and 650 seconds.

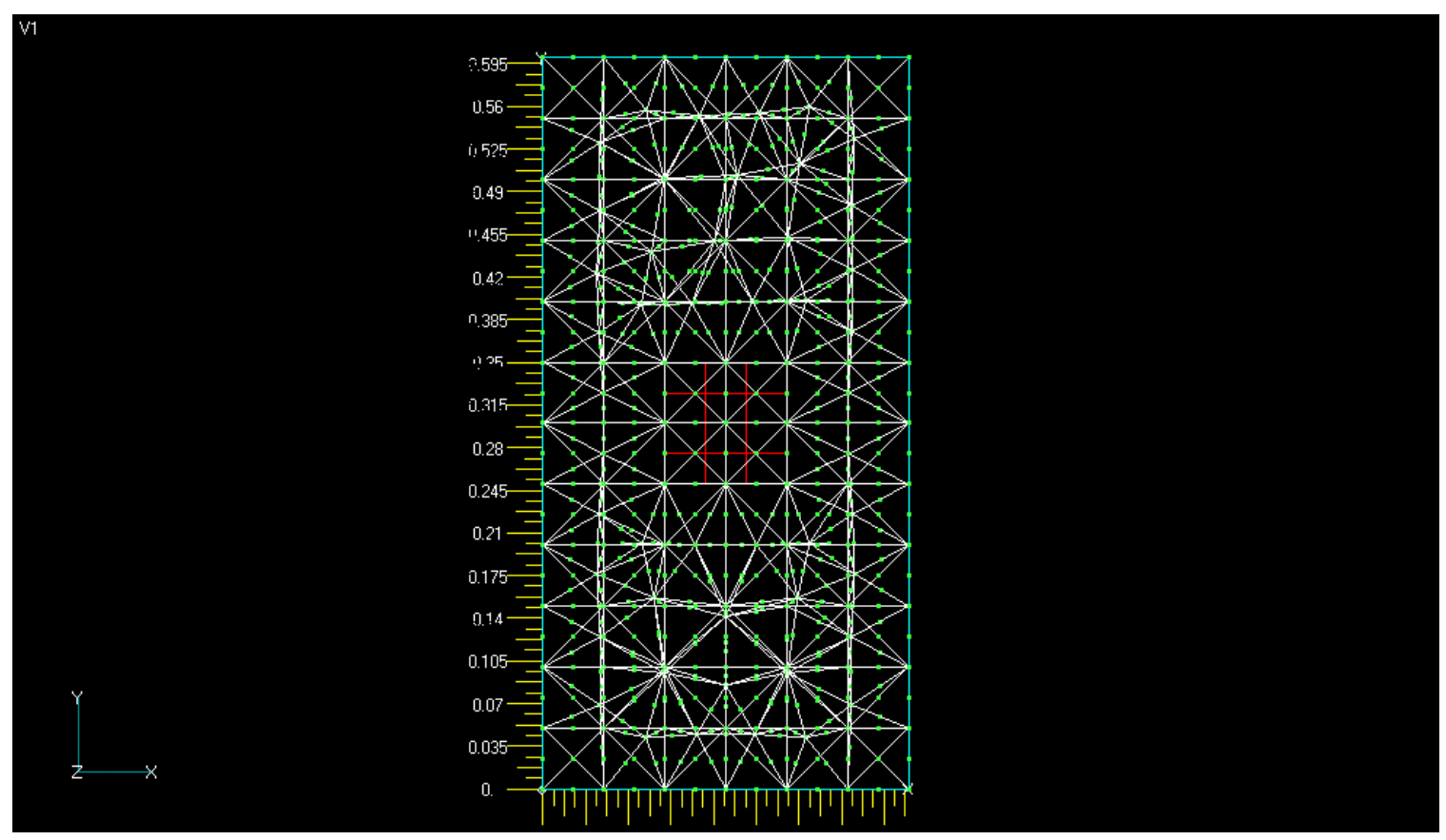

Figure 6.8: FE mesh of wearing surface layer with embedded debond.

\subsection{RESULTS AND DISCUSSION}

Even though the model in Figure 6.9 has twice the mesh size as the model in

Figure 6.10 , they have a pretty similar temperature profiles at time equal to 350 seconds. 
After 650 seconds, both profiles are still relatively close to each other. This indicates that the solution has achieved convergence. All other FE models generated from this point onward utilized the smaller $(0.025 \mathrm{~m})$ mesh size.

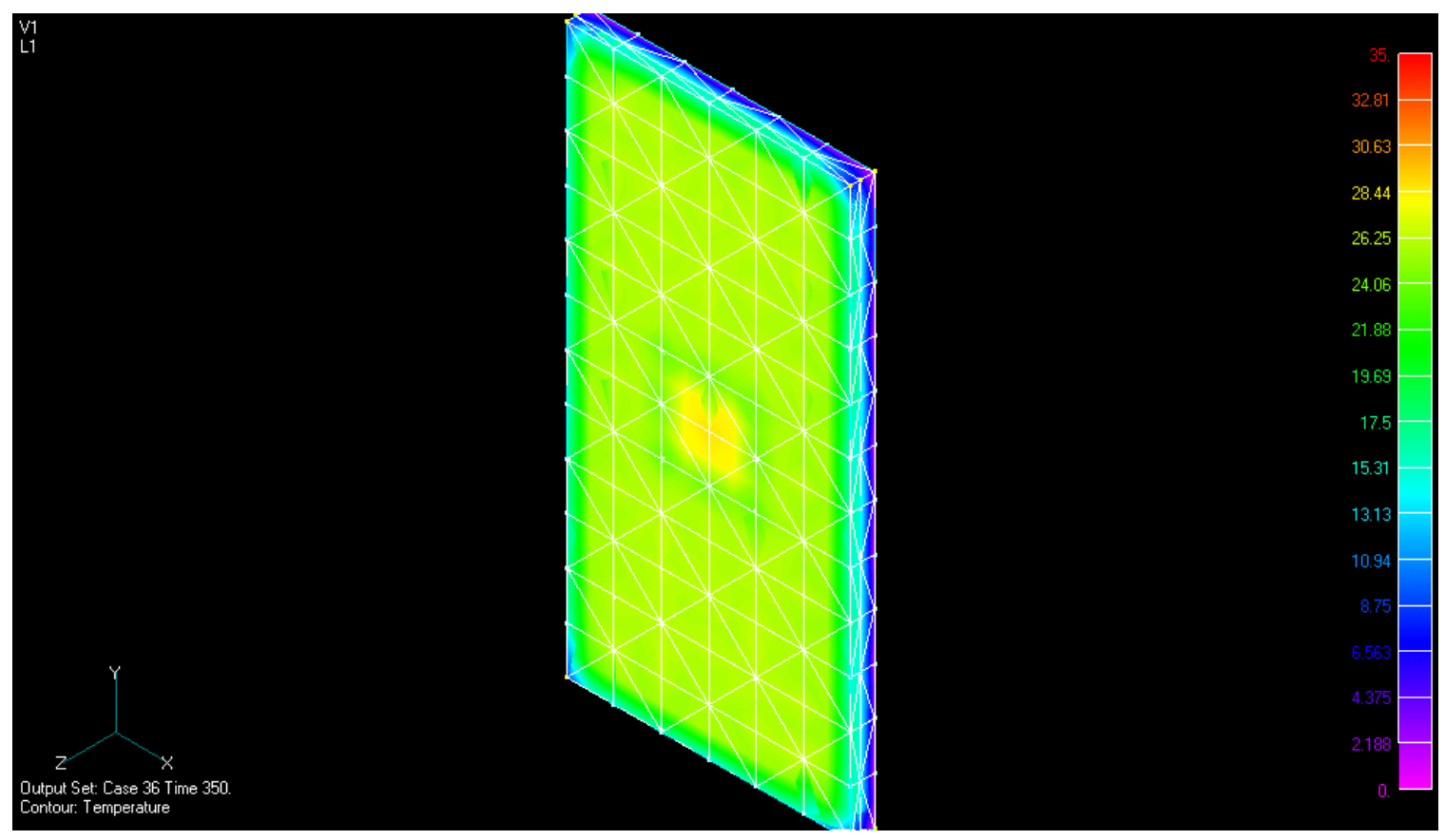

Figure 6.9: Temperature profile for a $0.1 \mathrm{~m} \times 0.1 \mathrm{~m} \times 0.0025 \mathrm{~m}$ air-filled debond with $0.05 \mathrm{~m}$ mesh, $\mathrm{t}=350 \mathrm{~s}$.

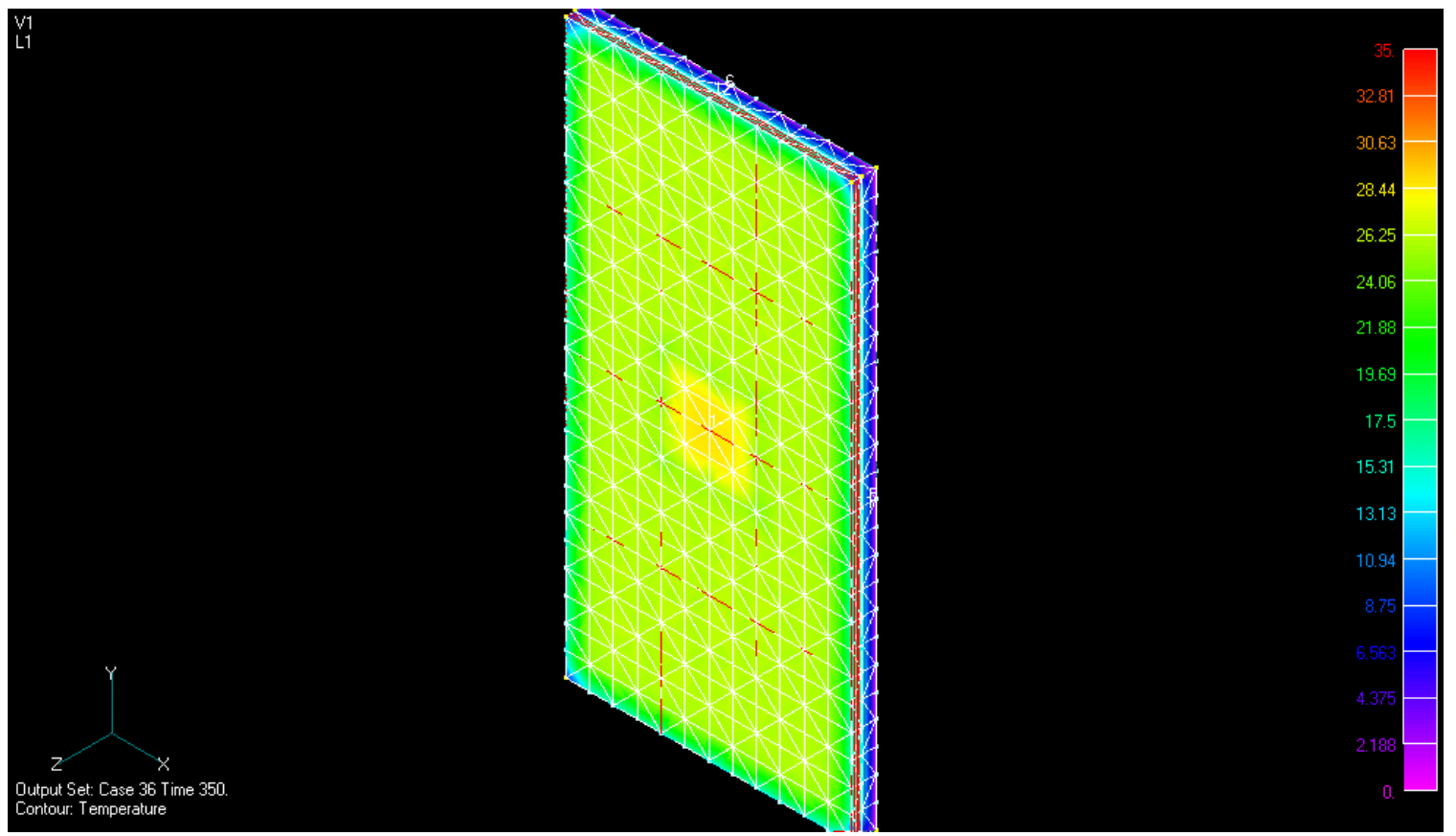

Figure 6.10: Temperature profile for a $0.1 \mathrm{~m} \times 0.1 \mathrm{~m} \times 0.0025 \mathrm{~m}$ air-filled debond with $0.025 \mathrm{~m}$ mesh, $\mathrm{t}=350 \mathrm{~s}$. 


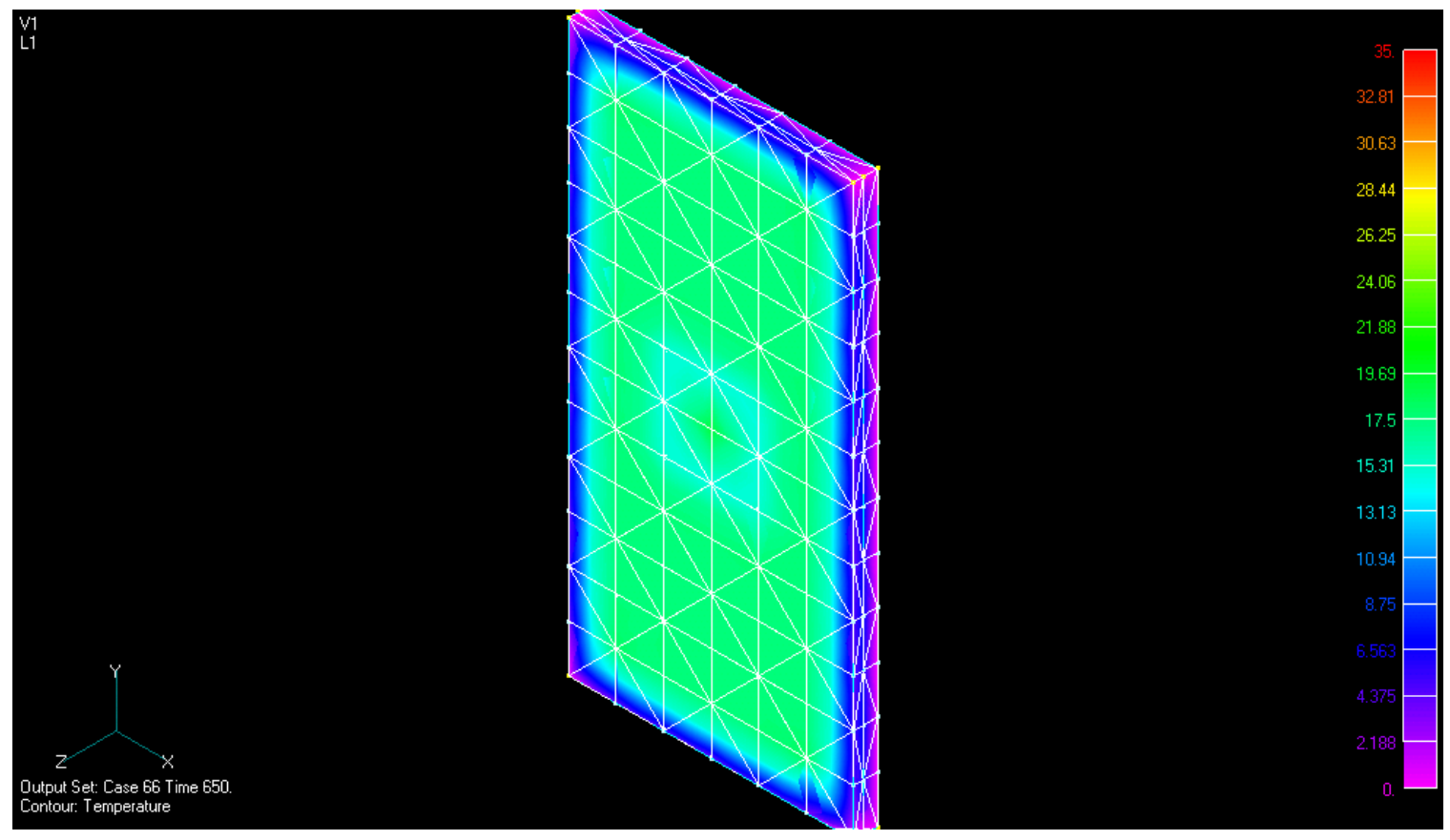

Figure 6.11: Temperature profile for a $0.1 \mathrm{~m} \times 0.1 \mathrm{~m} \times 0.0025 \mathrm{~m}$ air-filled debond with $0.05 \mathrm{~m}$ mesh, $\mathrm{t}=650 \mathrm{~s}$.

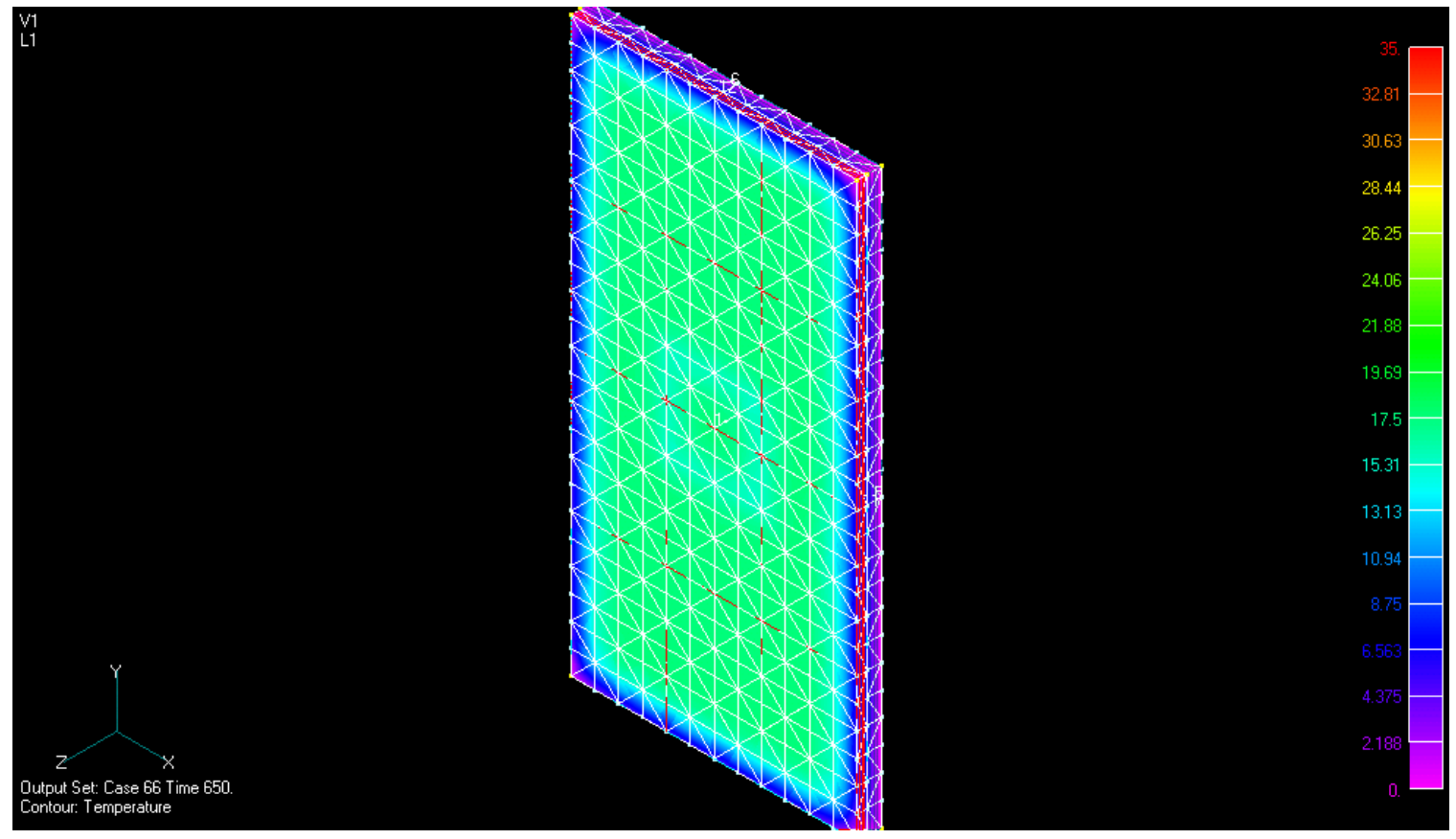

Figure 6.12: Temperature profile for a $0.1 \mathrm{~m} \times 0.1 \mathrm{~m}$ x $0.0025 \mathrm{~m}$ air-filled debond with $0.025 \mathrm{~m}$ mesh, $\mathrm{t}=650 \mathrm{~s}$. 
Notice in Figure 6.10, the model exhibits a hotter region on the surface of the defects. That is consistent with the IRT image for an air-filled debond. Close inspection of FE results for all other $0.1 \mathrm{~m} \times 0.1 \mathrm{~m}$ air-filled debond with various thicknesses reveal that as the thickness of the debond decreases, the amount of heat trapped above the debond decreases as well (Figures 6.13 and 6.14).

The surface temperature difference between the debonded and the defect-free area are recorded and plotted using Microsoft Excel (Figure 6.15). The Excel plot shows that the theoretical temperature changes are very much similar to that of actual temperature changes over time (Figure 5.29). However, the upward trend in Figure 5.29 lasted much longer than in case of the FE model shown in Figure 6.15. This could be due to the fact that Figure 5.29 (experimental result) is for larger defect sizes (in plan).

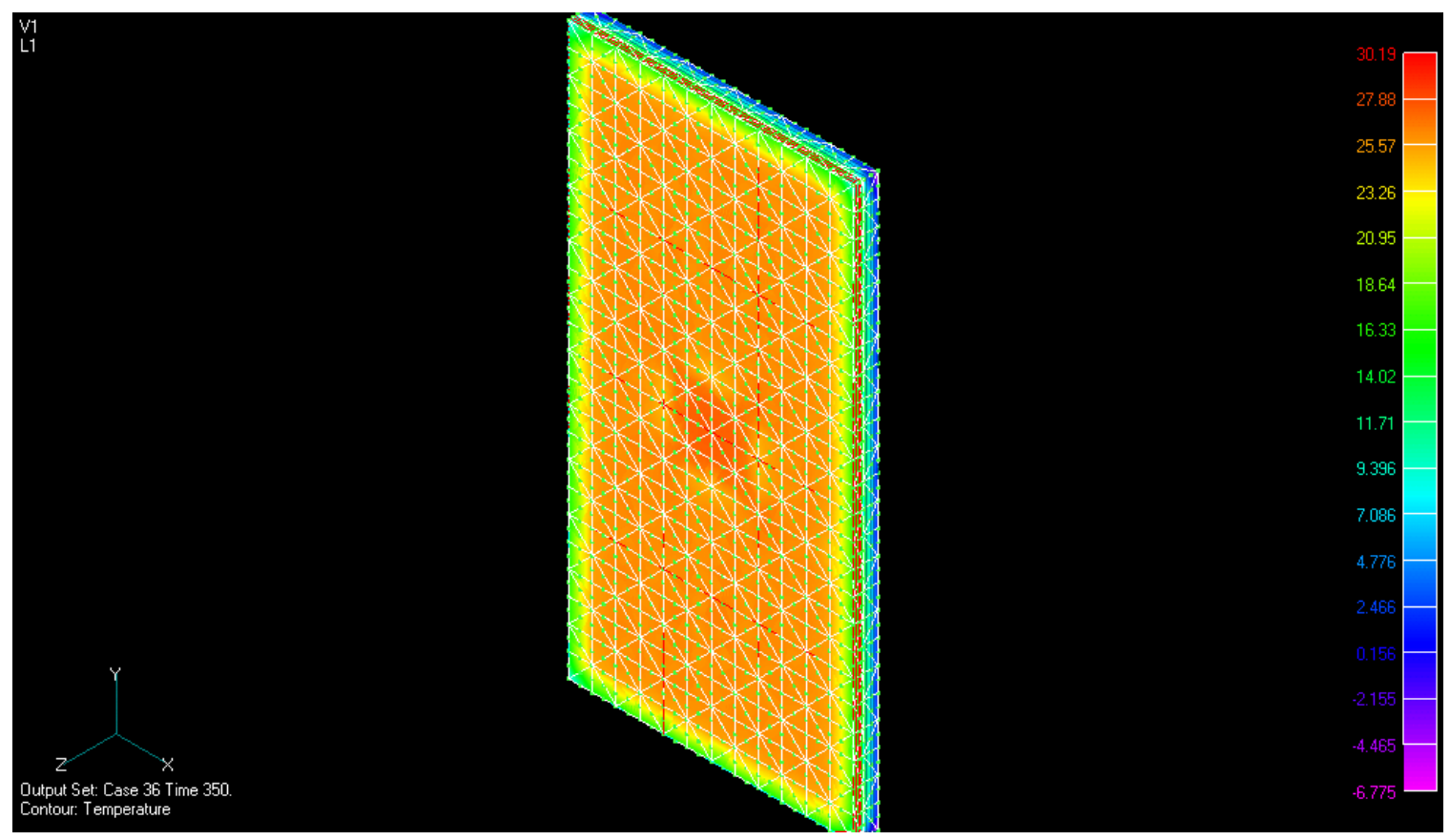

Figure 6.13: Temperature profile for a $0.1 \mathrm{~m} \times 0.1 \mathrm{~m} \times 0.0025 \mathrm{~m}$ air-filled debond with $0.025 \mathrm{~m}$ mesh, $\mathrm{t}=350 \mathrm{~s}$. 


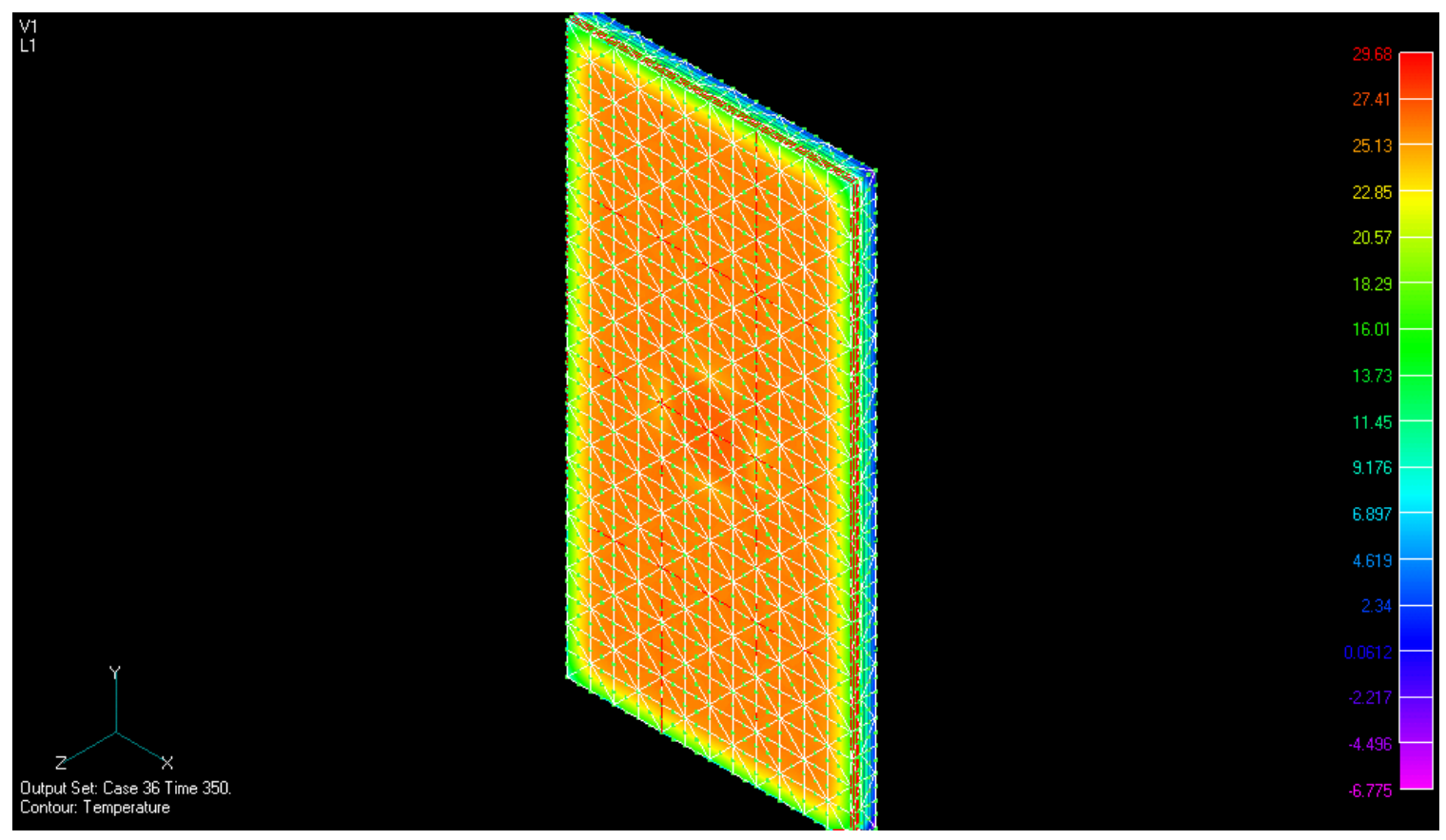

Figure 6.14: Temperature profile for a $0.1 \mathrm{~m} \times 0.1 \mathrm{~m}$ x $0.0015 \mathrm{~m}$ air-filled debond with $0.025 \mathrm{~m}$ mesh, $\mathrm{t}=350 \mathrm{~s}$.

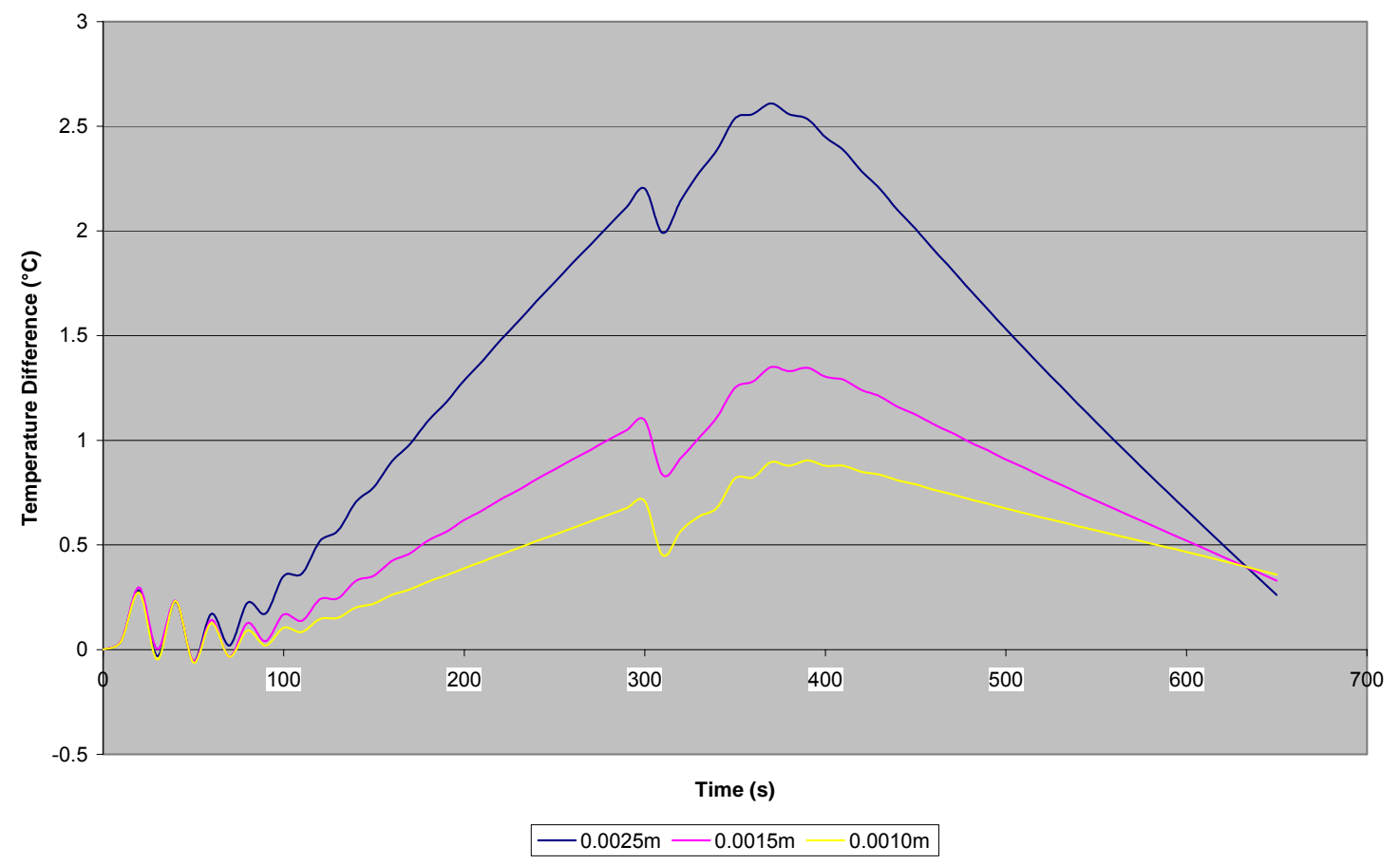

Figure 6.15(a): Temperature profile of various $0.1 \mathrm{~m} \times 0.1 \mathrm{~m}$ air-filled debonds minus defect-free. 


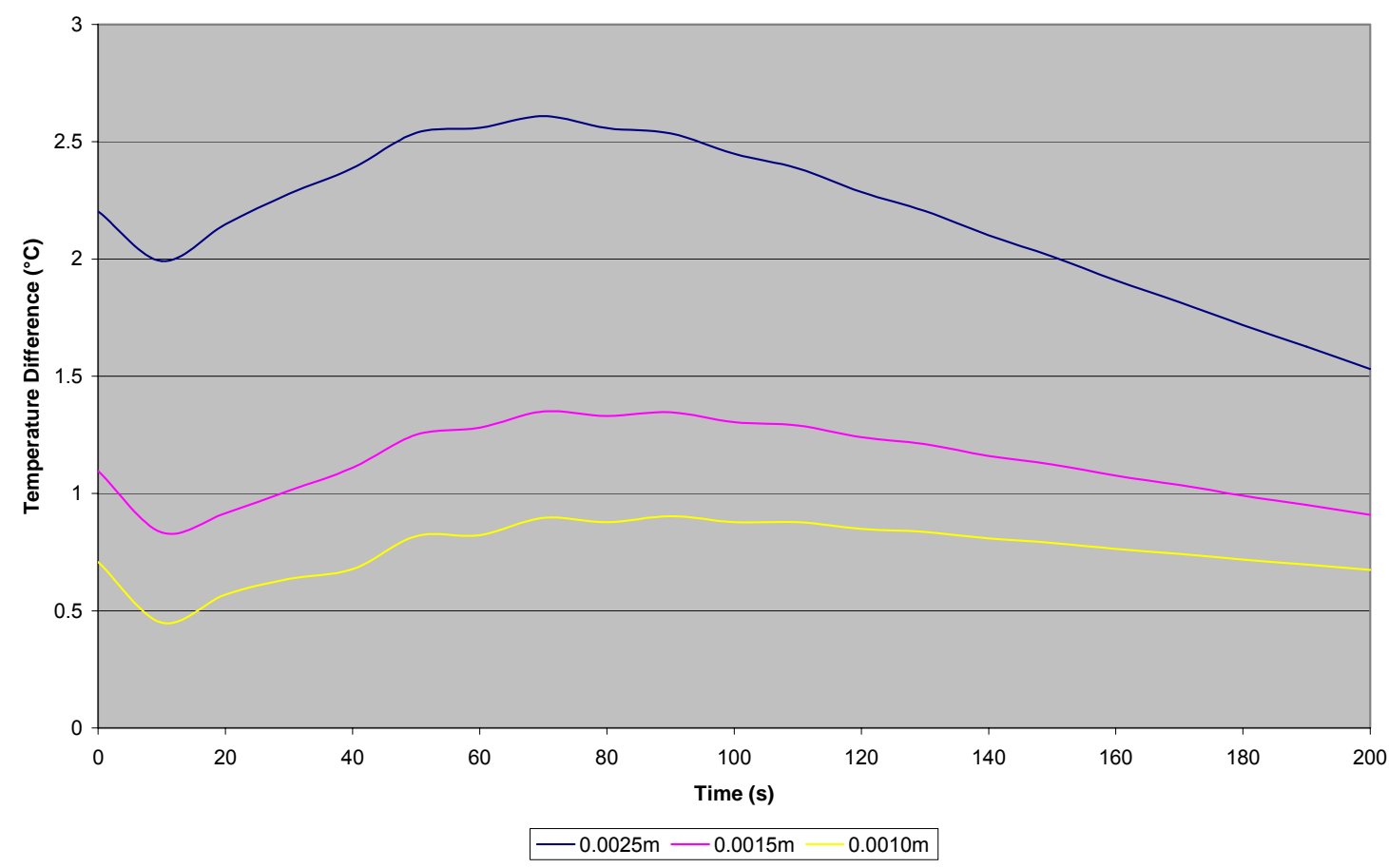

Figure 6.15(b): Temperature profile of various $0.1 \mathrm{~m} \times 0.1 \mathrm{~m}$ air-filled debonds minus defect-free (after removal of heat, 200 seconds comparison).

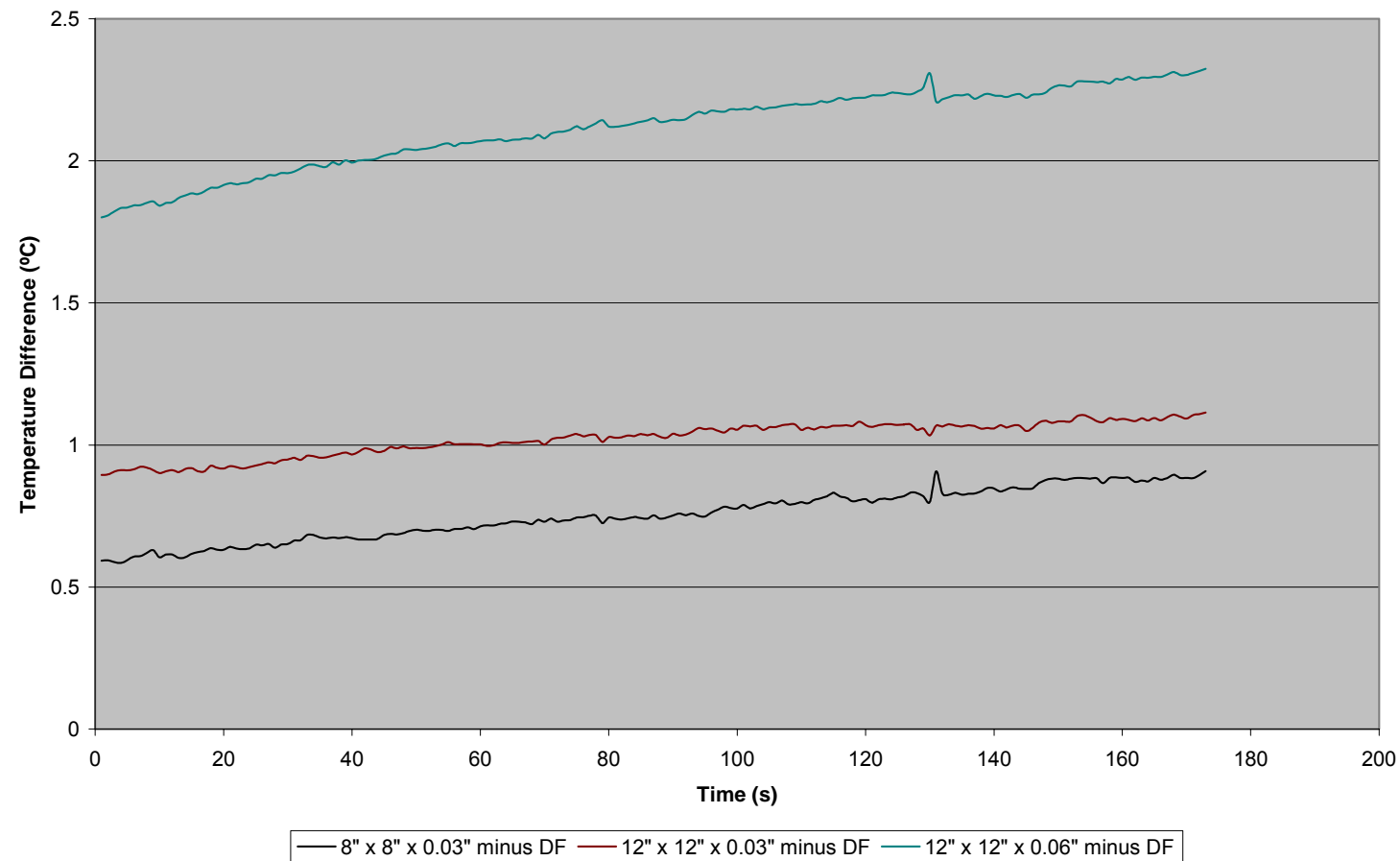

Figure 5.29: Temperature profile for defective minus defect-free for various air-filled debonds. 
Notice in Figure 6.15(a), the temperature difference seems to be converging after 650 seconds. On the other hand, a quick look at Figure 6.11 shows that though the center of the debond area and the defect-free area are indeed leveling temperature wise, there is a distinct "ring" around the boundary of the debond. This phenomenon is not common in actual IRT test in the laboratory. The temperature difference in the laboratory setting for a $0.09 "(0.0025 \mathrm{~m})$ thick air-filled debond is slightly lower than $2.5^{\circ} \mathrm{C}$ (Figure 5.31) while it is slightly higher than $2.5{ }^{\circ} \mathrm{C}$ theoretically. Furthermore, the $0.03 "(0.00076 \mathrm{~m})$ and $0.06 ”(0.0015 \mathrm{~m})$ air-filled debonds are hovering around $1{ }^{\circ} \mathrm{C}$ difference (Figure 5.29) while it is also $1{ }^{\circ} \mathrm{C}$ difference theoretically (Figure 6.15b).

The temperature difference for $0.05 \mathrm{~m} \times 0.05 \mathrm{~m}$ air-filled debonds is slightly smaller than that of $0.1 \mathrm{~m} \times 0.1 \mathrm{~m}$ (Figure 6.16). It is common knowledge in IRT imaging that a smaller size defect does indeed produce a lower surface temperature difference.

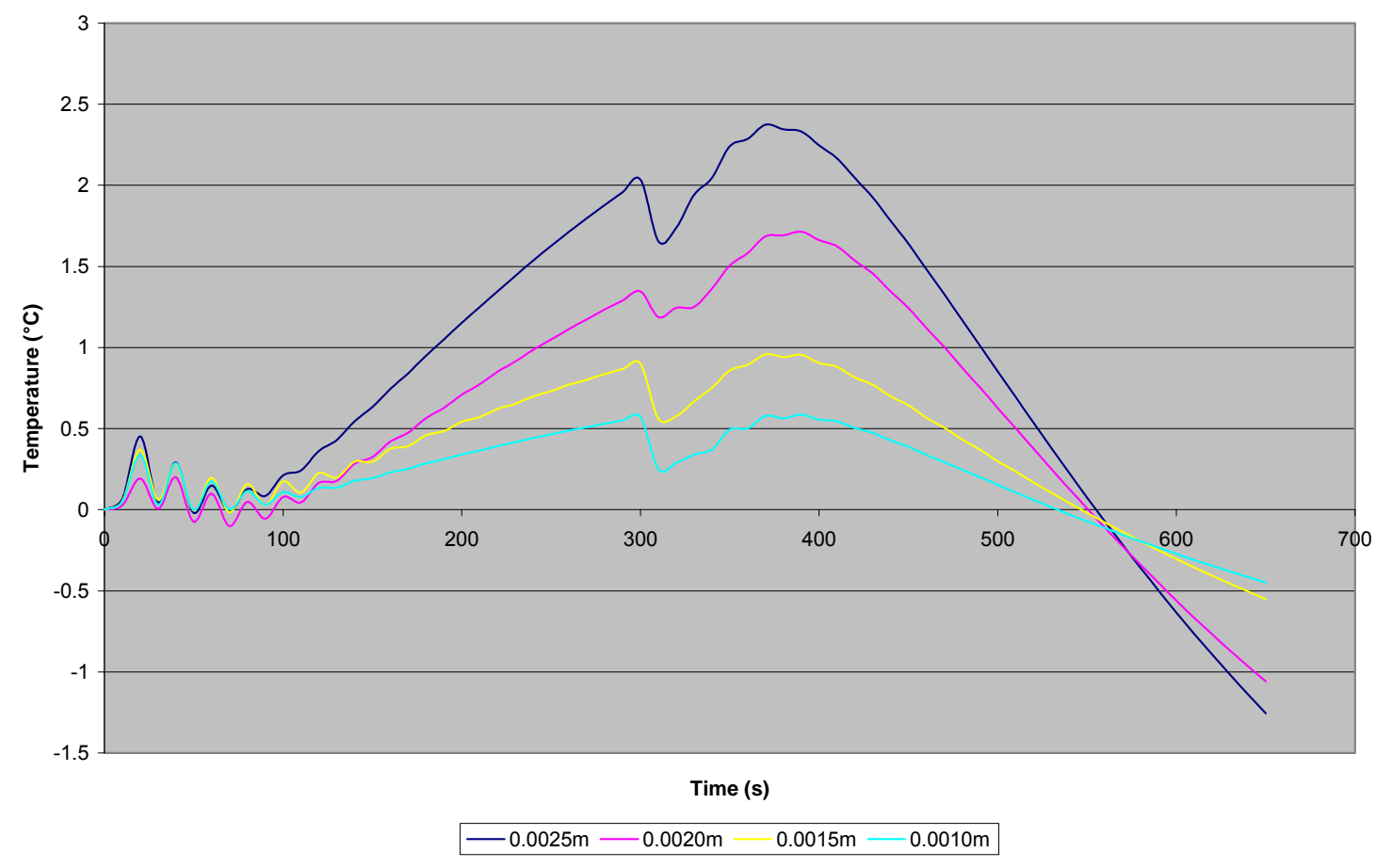

Figure 6.16: Temperature profile of various $0.05 \mathrm{~m}$ x $0.05 \mathrm{~m}$ air-filled debonds minus defect-free. 
As seen in Figure 6.17, the FE model for the water-filled debond clearly exhibits a lower surface temperature due to a much higher thermal conductivity. At the same time, water has a high specific heat value of $4180 \mathrm{~J} \mathrm{~kg}^{-1}{ }^{\circ} \mathrm{C}^{-1}$ and retains heat much better than air, thus resulting in a higher temperature than the surrounding after a long period of time (Figure 6.18). Once again, results from water-filled debond FE model shows the characteristic pertaining to a water-filled debond. The temperature of the debond as well as the defect-free area was noted and plotted using Microsoft Excel (Figure 6.19). Unfortunately, there are no actual data of 4" x 4 " (roughly $0.1 \mathrm{~m} \times 0.1 \mathrm{~m}$ ) water-filled debonds available for direct comparison with Figure 6.19. However, the temperature profile of a theoretical water-filled debond does exhibit some characteristics that could be found on actual water-filled debond. As seen in Figure 5.33 and Figure 6.19 (especially the region after 300 seconds), the curves show a general upward trend before

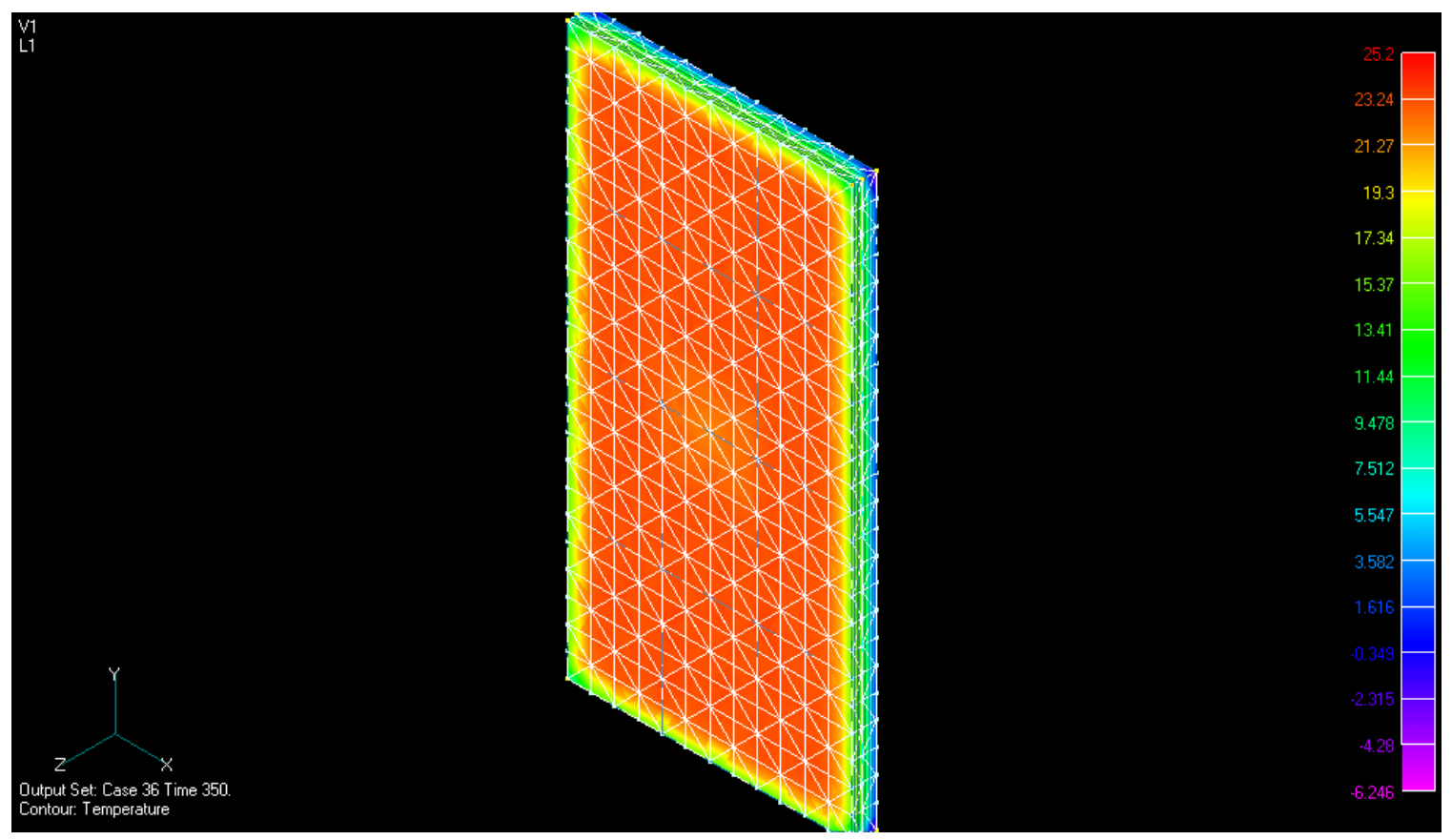

Figure 6.17: Temperature profile for a $0.1 \mathrm{~m} \times 0.1 \mathrm{~m} \times 0.0025 \mathrm{~m}$ water-filled debond with $0.025 \mathrm{~m}$ mesh, $\mathrm{t}=350 \mathrm{~s}$. 


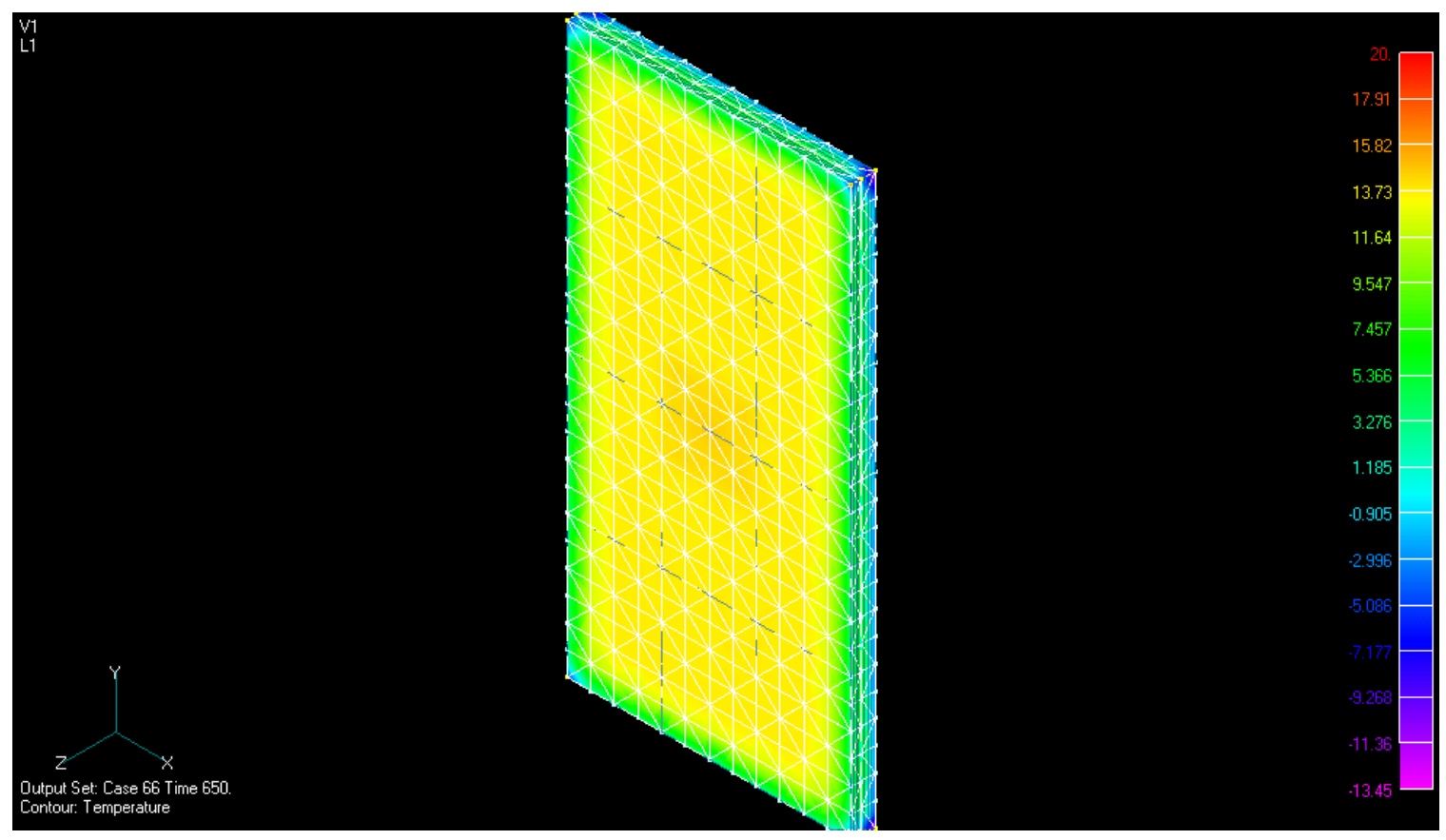

Figure 6.18: Temperature profile for a $0.1 \mathrm{~m} \times 0.1 \mathrm{~m} \times 0.0025 \mathrm{~m}$ water-filled debond with $0.025 \mathrm{~m}$ mesh, $\mathrm{t}=650 \mathrm{~s}$.

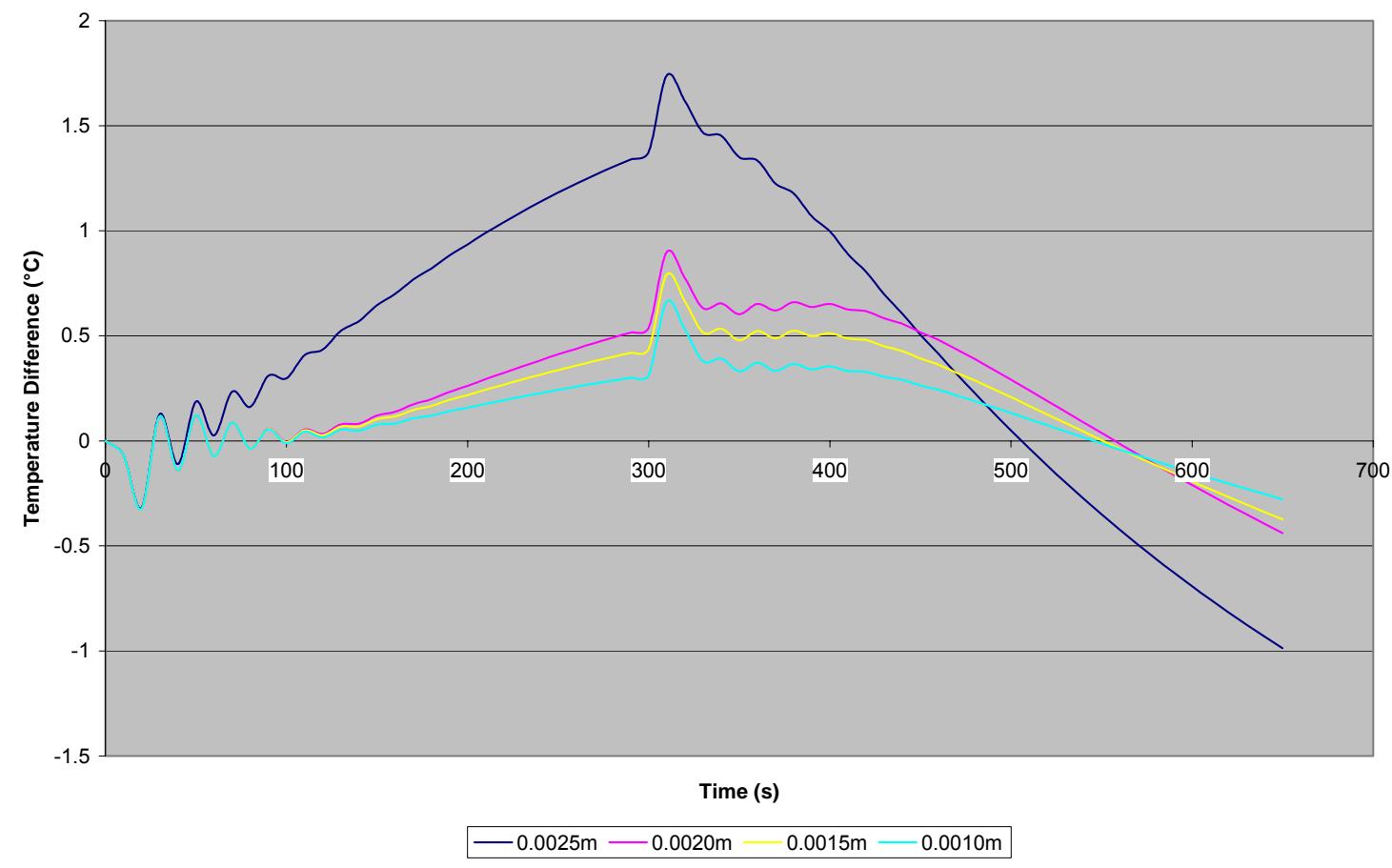

Figure 6.19: Temperature profile of defective minus defect-free for $0.1 \mathrm{~m}$ x $0.1 \mathrm{~m}$ water-filled debonds. 


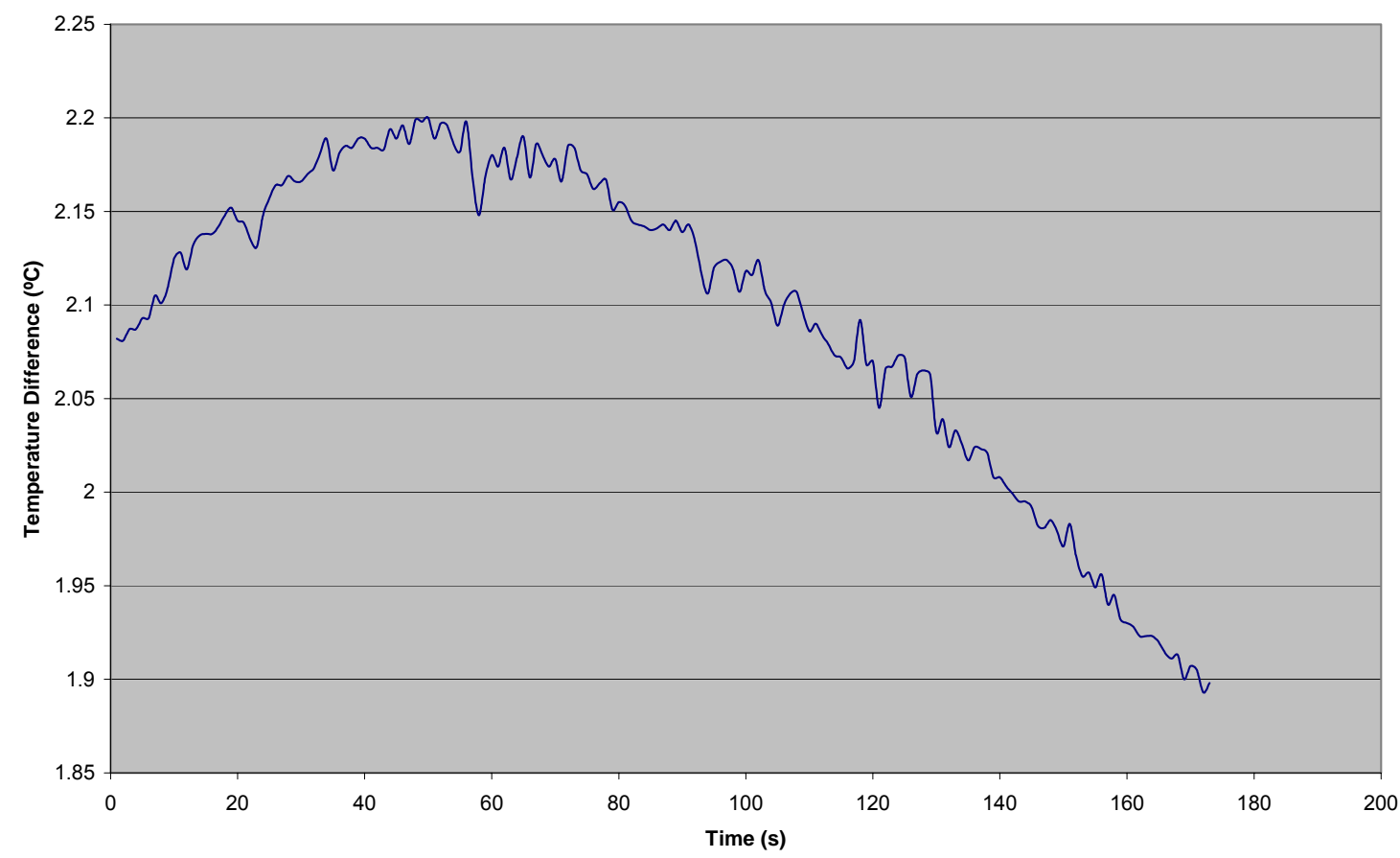

Figure 5.33: Temperature profile of defective minus defect-free for 8 " × 8 " x 0.09 " water-filled debond.

reaching a maximum value followed by a decreasing trend. Based on the results from the air-filled debond models, the water-filled debond model should in theory work as well as the air-filled debond model, but this issue needs to be investigated further by using identical defect sizes in comparing the experimental results with the theoretical predictions from the finite element mode.

In Figure 6.20, it is clear that the defects partially filled with water exhibit some rather unusual results. The temperature profile for all the cases (with different saturation levels) are identical. Even though the model assumes a horizontal deck with air layer always over the water layer in case of partially saturated debonds, the thickness of the air layer is reduced with higher water saturation level, and the model should show some difference. Thus, it can be concluded that the partially water-filled debond model is not 
valid at this point even though it was an evolution from the working air-filled debond model. This model for partially saturated case needs further investigation.

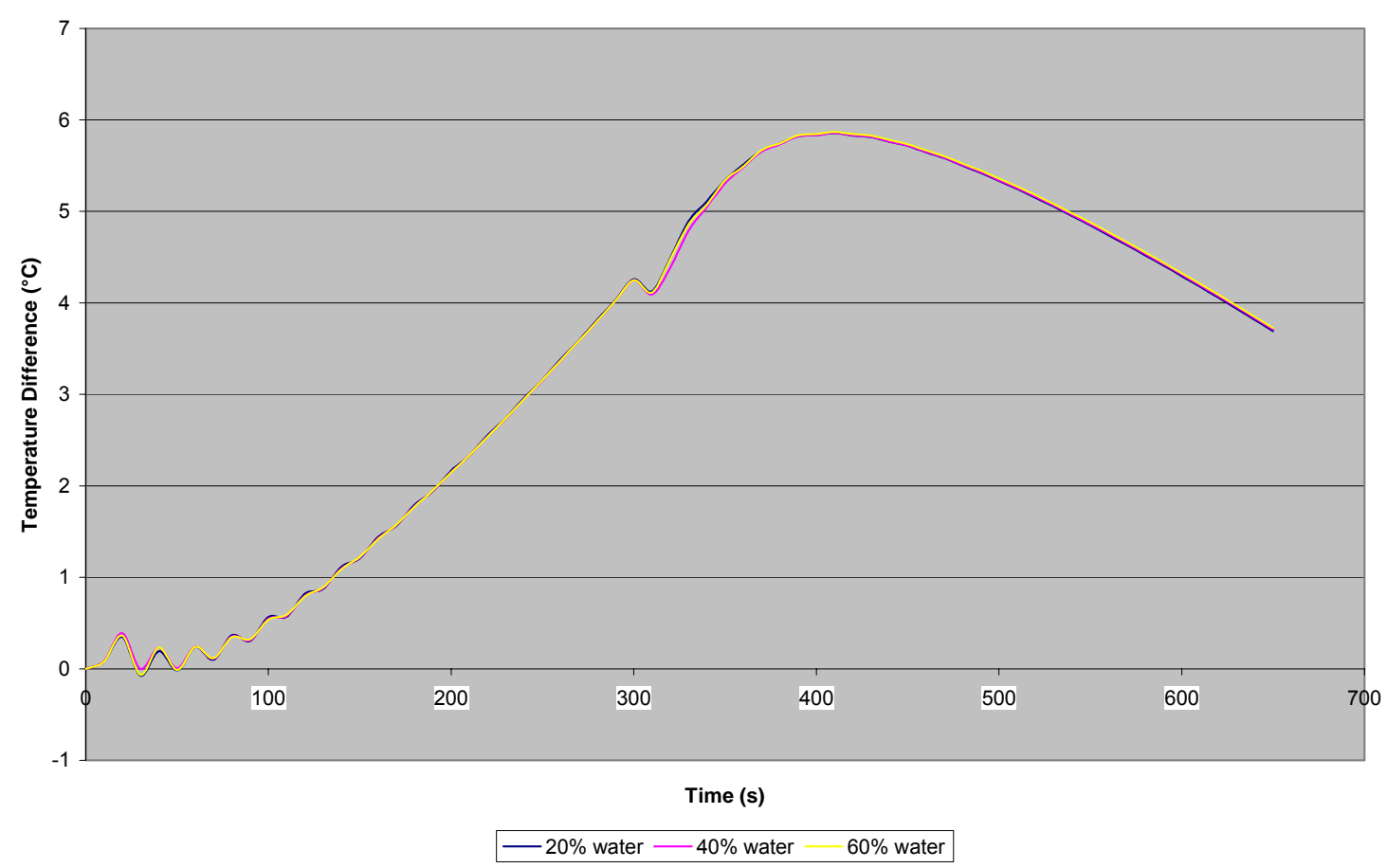

Figure 6.20: Temperature profile of defective minus defect-free for $0.1 \mathrm{~m} \mathrm{x} 0.1 \mathrm{~m}$ partially water-filled debonds.

\subsection{CONCLUSIONS}

Based on the comparison between the actual laboratory data and the theoretical data generated using finite element model, it is clear that the FE model has an acceptable accuracy in describing the actual heat transfer process in FRP decks with air-filled debonds. Future studies should focus on improving the modeling around the boundary of debonds to better describe the actual heat transfer of an air-filled debond situation.

The fully water-filled debond models generated in this study have shown promising characteristics. However, the finite element results for partially water-filled debonds are not valid, and future effort should focus on successfully modeling such cases since they may describe some of the field conditions more accurately. 


\section{Chapter 7}

\section{EXPERIMENTAL RESULTS FROM GROUND PENETRATING}

\section{RADAR}

This chapter discusses in detail all the GPR tests conducted in this study using 2.0GHz air-launched and $1.5 \mathrm{GHz}$ ground-coupled antennas. The first GPR test was conducted by placing a 3' x 2' wearing surface module on the top of a 3' x 6' low-profile (4" thick) FRP bridge deck with a water-filled defect planted underneath the 3/8" thick

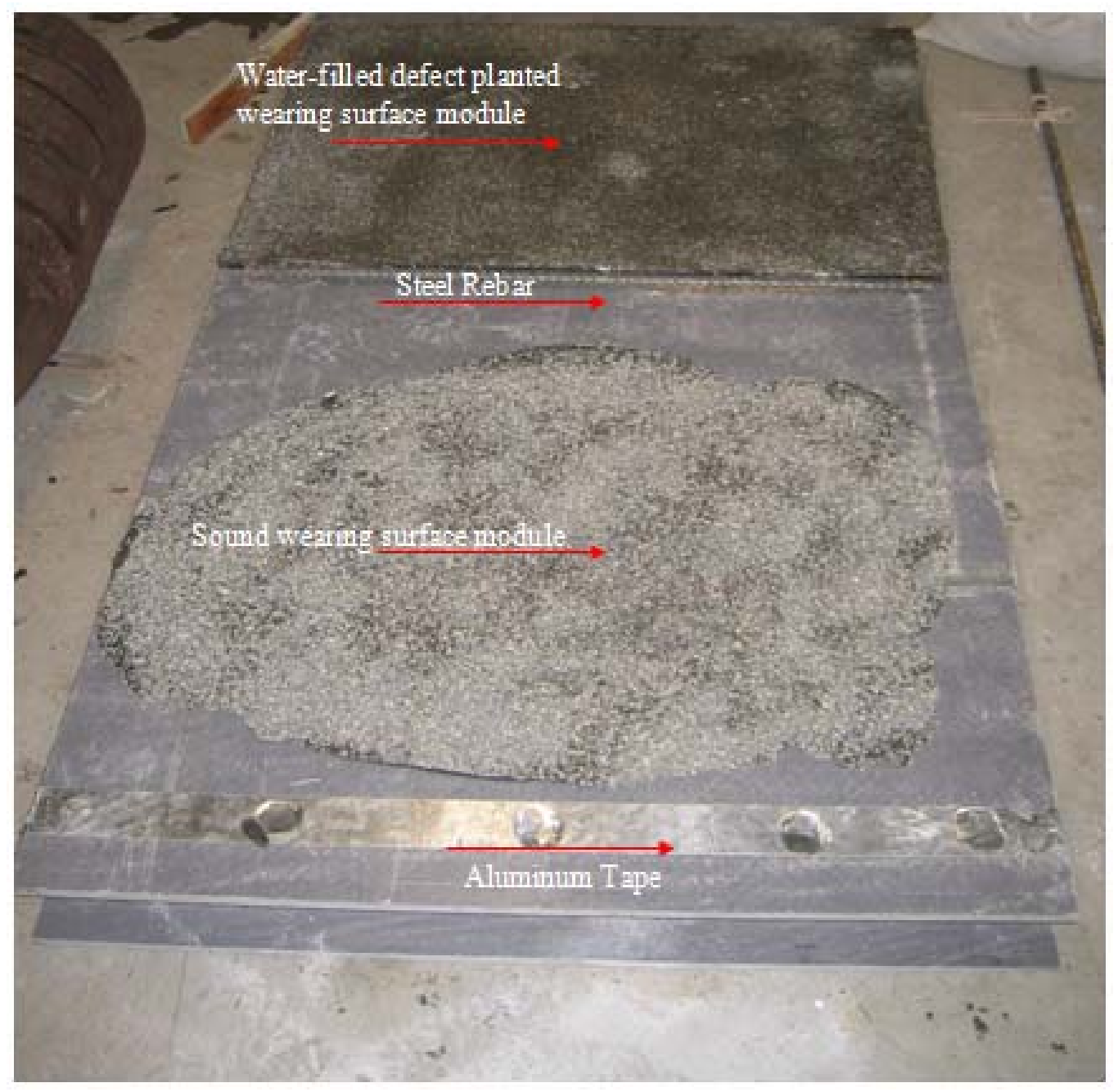

Figure 7.1: The setup of the preliminary GPR test. 
wearing surface. Adjacent to it (on the same FRP deck), another defect-free wearing surface module was placed as shown in Figure 7.1. To simplify the post-processing, an aluminum tape was placed right before the sound wearing surface module and a steel rebar was placed right before the water-filled wearing surface module (Figure 7.1). The entire setup was then tested using the $2 \mathrm{GHz}$ air-launched GPR system in the laboratory, with the signals recorded and processed later by using the GPR processing software (RADAN 5.0) provided by the GPR manufacturer. The GPR data was also converted to ASCII format for signal comparisons using EXCEL plots.

RADAN 5.0 was used to process the collected GPR data. After applying a range gain of 5 as well as Finite Impulse Response (FIR) filters, the RADAN software resulted in the GPR scan shown in Figure 7.2.

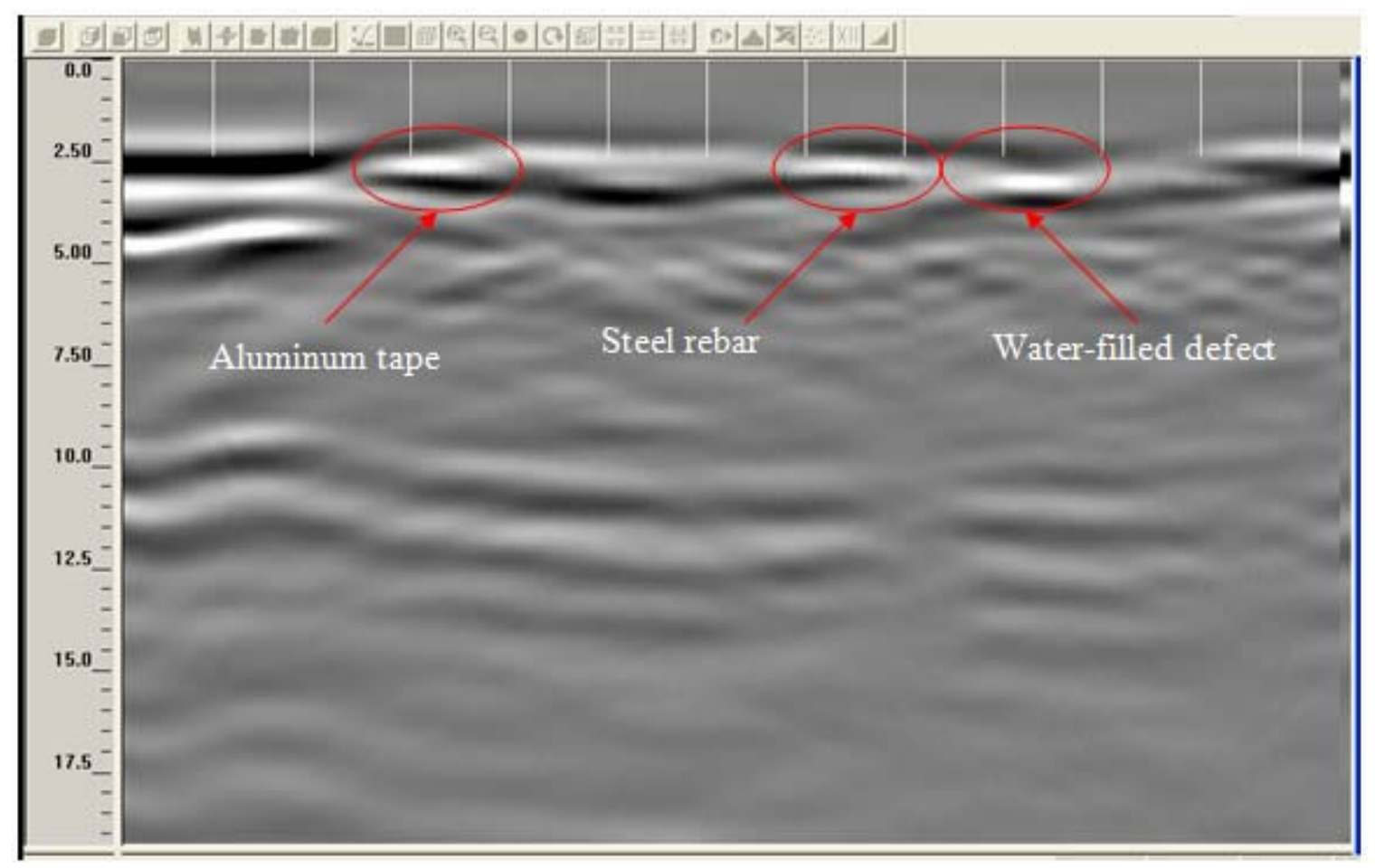

Figure 7.2: GPR scan of the test deck. 
As indicated in Figure 7.2, the aluminum tape, steel rebar, and the water-filled defect could be detected very clearly. This is because these objects result in higher signal reflection echoes in the GPR scan as shown by the bright white strips. It was later verified through the distance measurement that the position of the objects were accurately stated on the plot. To take a step further, GPR signals were extracted from each of the region listed on the plot above, as well as the defect-free wearing surface region. The signals were plotted in Excel and are shown in Figure 7.3.

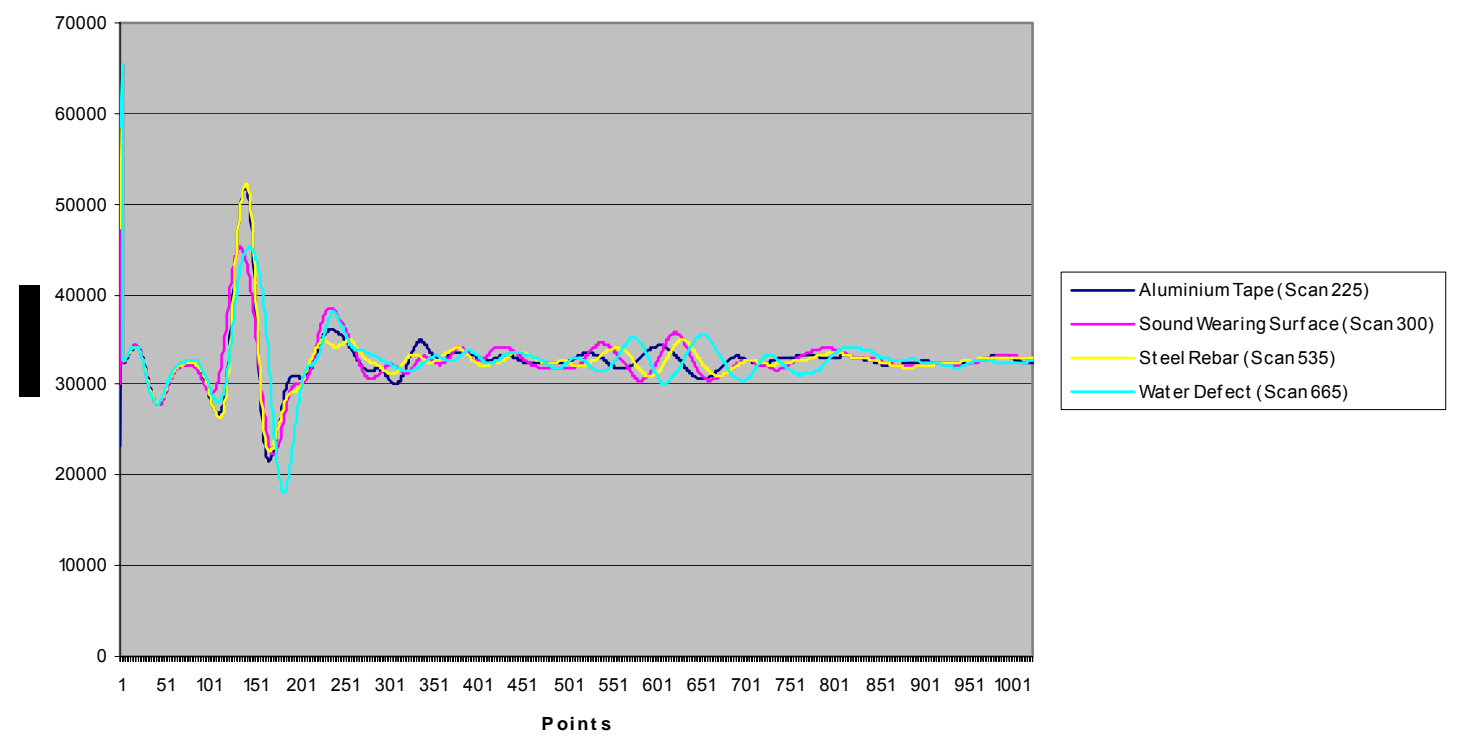

Figure 7.3: Processed signals as Excel plot.

The amplitude of the aluminum tape is almost the same as that of the steel rebar. They are both metallic objects with more or less same plan sizes, and equal reflection amplitudes from these objects indicate that the GPR system is working perfectly. The location of the water-filled defect was directly under the wearing surface, and the reflection from the top surface of the wearing surface and the reflection from water-filled defect overlapped to give rise to the resulting water-filled defect signal. When the defect- 
free signal was subtracted from the water-filled defect signal, the resulting difference (Figure 7.4) showed the presence of the water-filled defect very clearly.

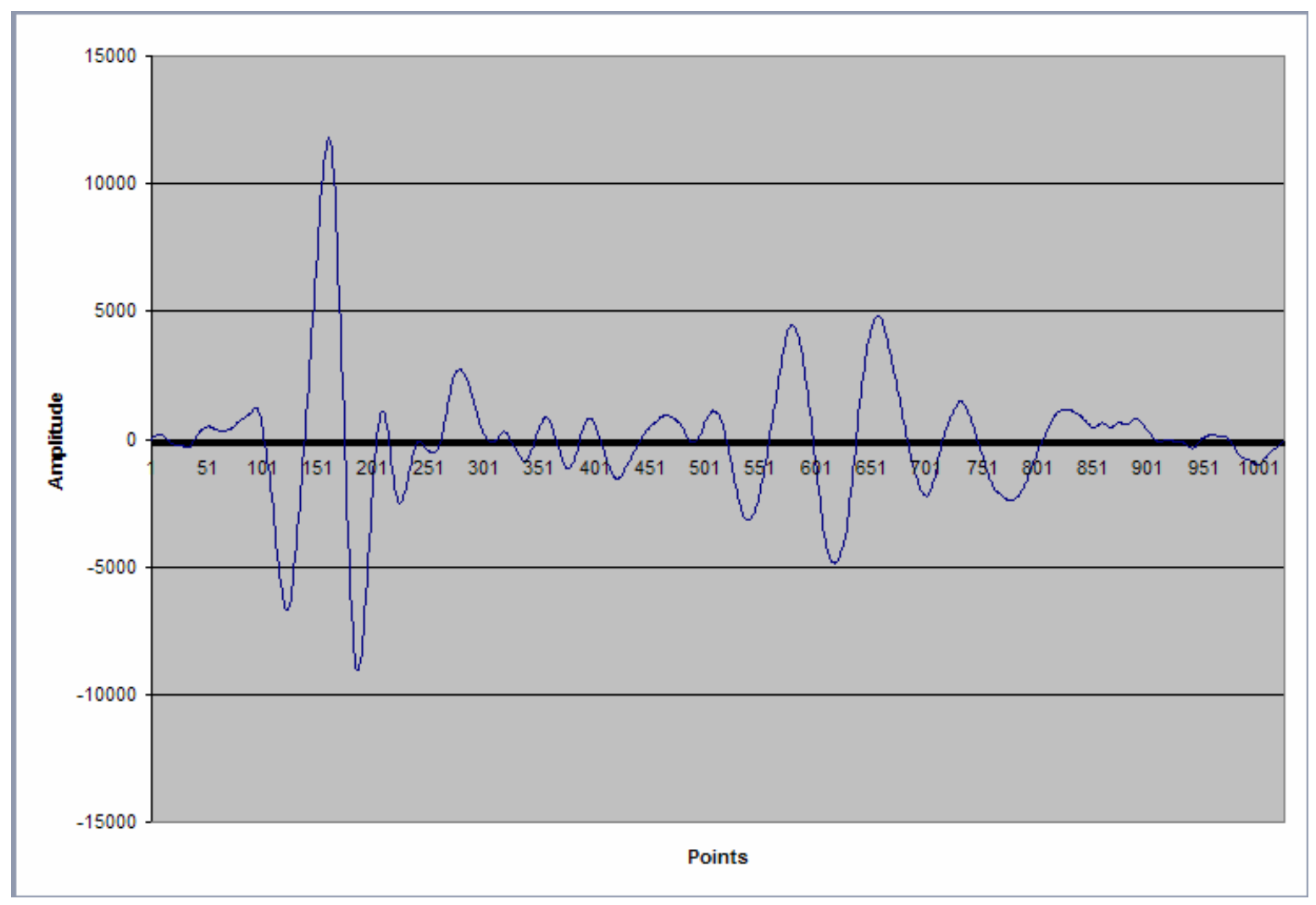

Figure 7.4: Result of subtraction of defect-free signal from water-filled signal.

\subsection{FULL SCALE GPR TEST}

\subsubsection{Detection of Water-filled and Air-filled Debonds}

The GPR system used in this study was the SIR-20 system manufactured by Geophysical Survey Systems, Inc. (GSSI), New Hampshire. The SIR-20 GPR system with the vehicle mount boom was assembled on a hand-wheel cart instead of a vehicle so that the experiments could be conducted inside a laboratory setting. Because of the long length of the assembled GPR scanning system, a wooden platform with plan dimensions of $30 \mathrm{ft} \times 9 \mathrm{ft}(9.14 \mathrm{~m} \times 2.74 \mathrm{~m})$ was constructed around the FRP test bridge deck with the wearing surface (Figure 7.5). The surface of the FRP bridge deck was level with the rest 


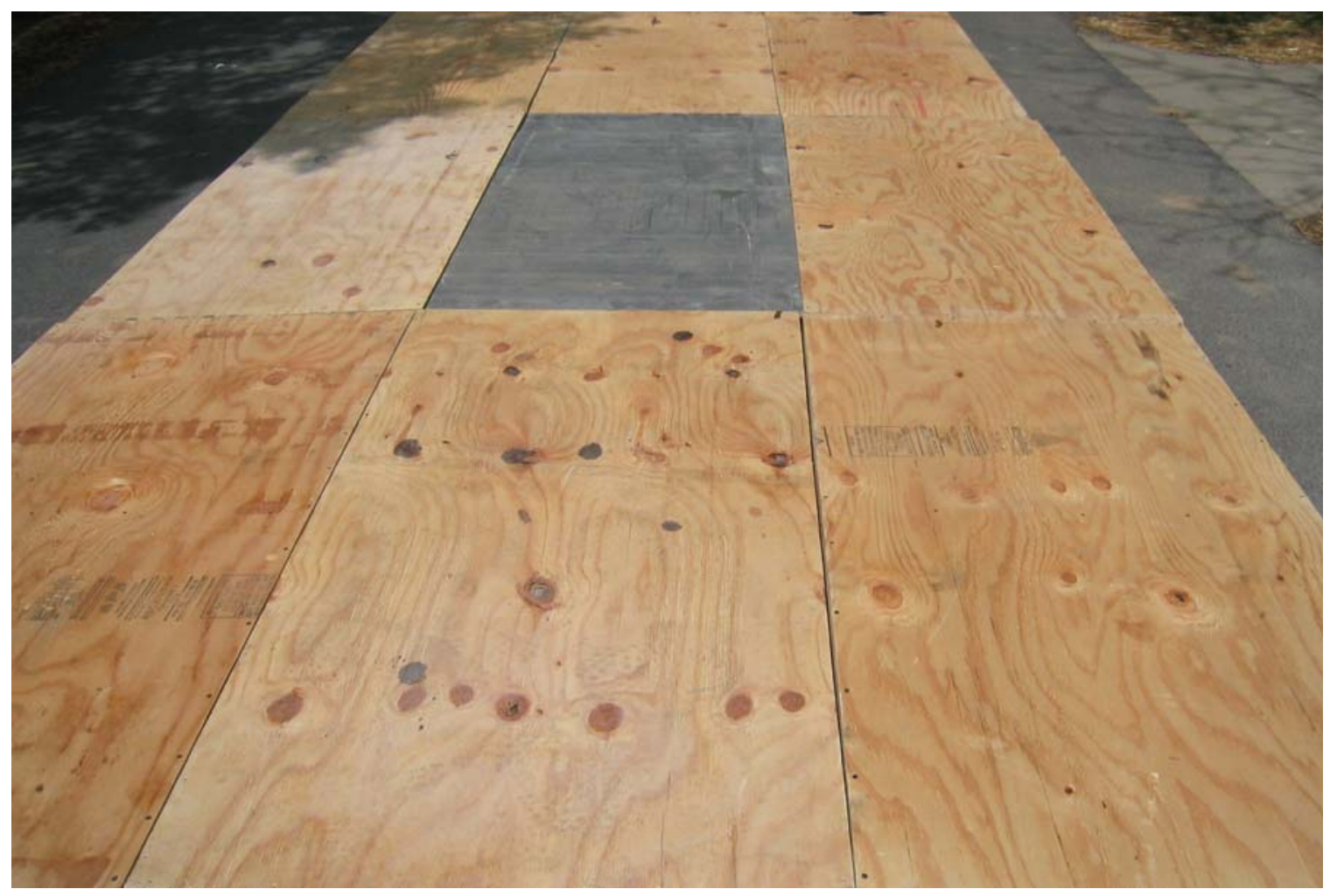

Figure 7.5: FRP Deck module with the wooden platform setup.

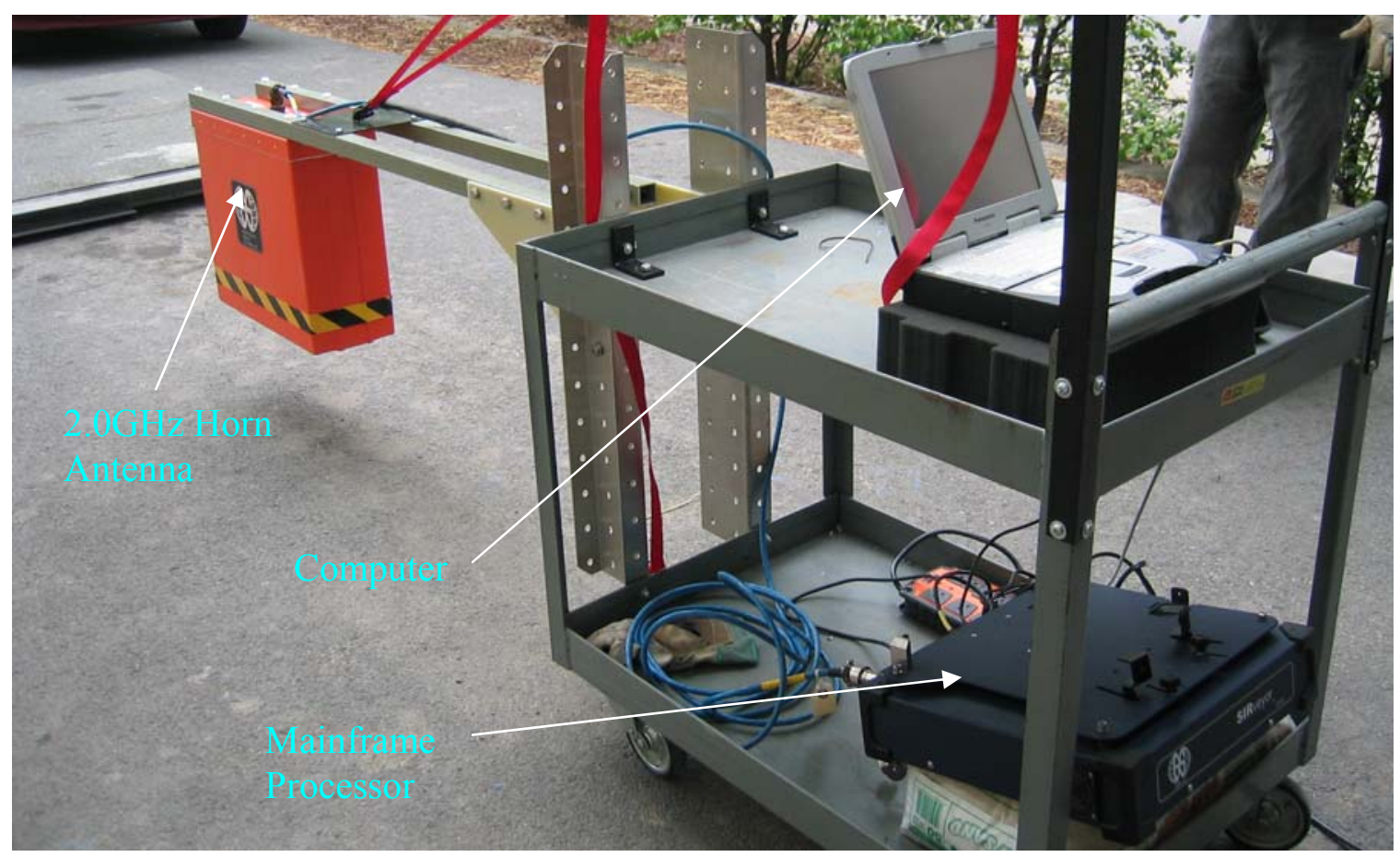

Figure 7.6: Hardware configuration of SIR-20 GPR system with $2.0 \mathrm{GHz}$ horn antenna. 
of the platform as can be seen in Figure 7.5. The hand-wheel cart housed the GPR mainframe processor as well as the computer, while an optical encoder based survey wheel was attached to the bottom of the cart itself. A $2.0 \mathrm{GHz}$ air-coupled horn antenna was attached to one end of the boom extension and the other end of the boom was bolted to the cart (Figure 7.6). In addition to the horn antenna, a $1.5 \mathrm{GHz}$ ground-coupled antenna was also used in the GPR experiments (Figure 7.7).

The GPR mainframe processor is used to excite the antenna and receive the collected data while the attached computer is used to configure the data acquisition settings and store the collected data. The same mainframe processor and the computer are used for both the $2.0 \mathrm{GHz}$ air-coupled horn antenna and the $1.5 \mathrm{GHz}$ ground-coupled antenna. These antennas were chosen since they offered the highest commercially available frequency in the air-coupled and ground-coupled categories, respectively. The higher the frequency, the shorter is the wavelength, and this results in better resolution of subsurface defects.

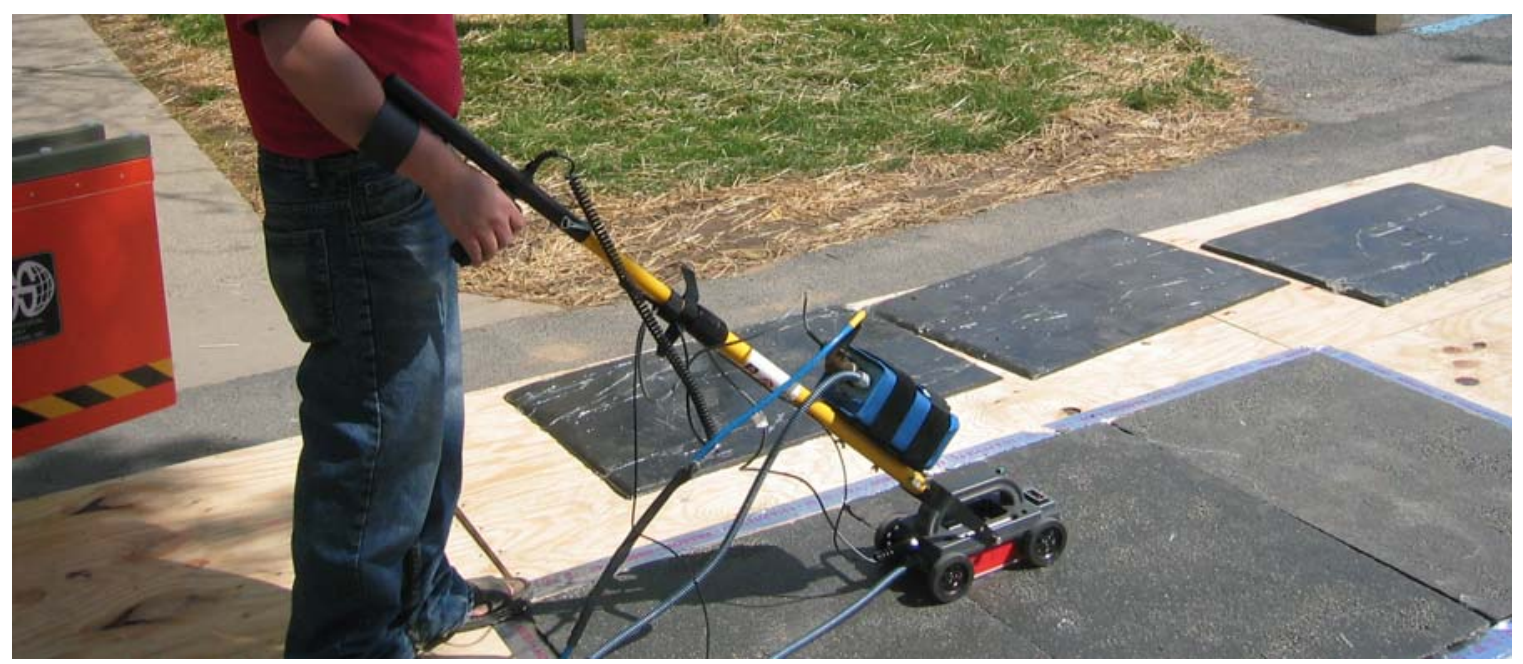

Figure 7.7: $1.5 \mathrm{GHz}$ ground-coupled antenna. 
The focus of this experiments using GPR was to evaluate GPR's ability to detect small subsurface defects. The defects were carefully designed and placed under the wearing surface modules. The 3' x 2' wearing surface module with defects were placed over the middle portion of the 3' x 6' FRP bridge deck, with two defect-free wearing surfaces (3' x 2' plan size) placed before and after it over the same deck. After scanning the deck setup using GSSI's SIR-20 unit along with the $2.0 \mathrm{GHz}$ air-coupled horn antenna as well as the $1.5 \mathrm{GHz}$ ground-coupled antenna, the radar data were processed using the available RADAN post-processing software provided by the GPR equipment manufacturer. For research purposes, the data range for this experiment was set to 5 nanoseconds to ensure a higher resolution at regions where the clarity of the data set is most crucial. At the same time, the system was set to collect 512 points (rather than 1024 points) for each scan so that the GPR system could be operated at a faster pace. Prior to the actual experiments, a scan of the wearing surface as well as a metal plate reflection was taken (using the air-launched antenna) and used to compute the dielectric constant of the wearing surface. The dielectric constant of the Transpo's wearing surface was computed as 4. This is an important piece of information as the SIR-20 system will need user input of the dielectric constant of the first medium encounter by GPR. The dielectric constant of the FRP deck was found to be higher $(\sim 10)$.

All subsequent GPR experimental data from the test FRP deck was collected using the survey wheel mode, which provides an accurate account of horizontal position in the scanning area. The survey wheel has an in-built optical encoder which provides distance information to the mainframe processor, and also enables the user to control the 
number of radar waveforms acquired per unit distance. The survey wheel data allows the user to visually pin-point potential defective areas.

In order to compare the data sets from different GPR tests later during the postprocessing phase of the experiment, all GPR data were collected using the same initial setting. Aluminum tapes were placed at the boundary of the FRP deck to mark the starting and ending points for each scan in order to provide a benchmark for postprocessing. Since aluminum tape reflects the entire electromagnetic wave energy incident on it, the amplitude of its reflection will be significantly higher than from other areas of the FRP deck (Figures 7.8 and 7.9). Also, as indicated by Figure 7.8, the $1.5 \mathrm{GHz}$ groundcoupled antenna has the sensitivity to pick up even the webs of the FRP deck which are not visible in the GPR scan using the $2.0 \mathrm{GHz}$ air-coupled antenna (Figure 7.9). This is because in case of the air-coupled antenna, a significant portion of the incident electromagnetic energy is reflected off of the wearing surface (air-wearing surface boundary). On the other hand, the ground-coupled antenna is able to transmit more energy into the deck, which results in deeper penetration.

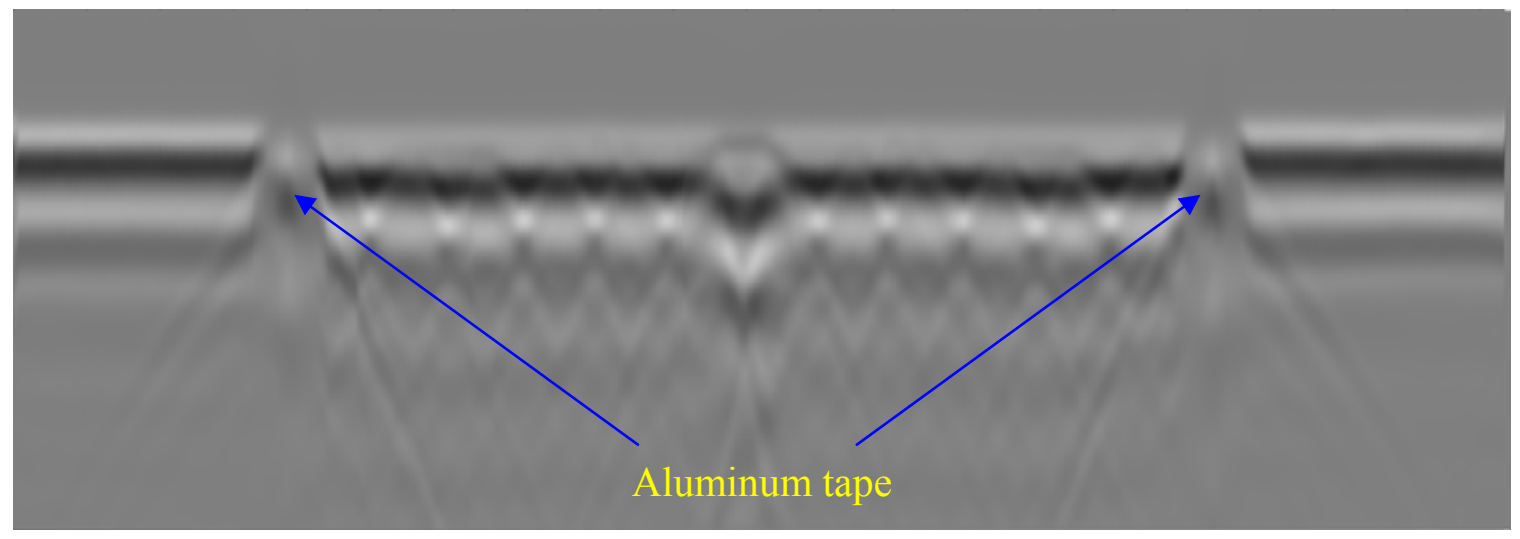

Figure 7.8: A typical GPR scan of an FRP deck using 1.5GHz ground-coupled antenna. 


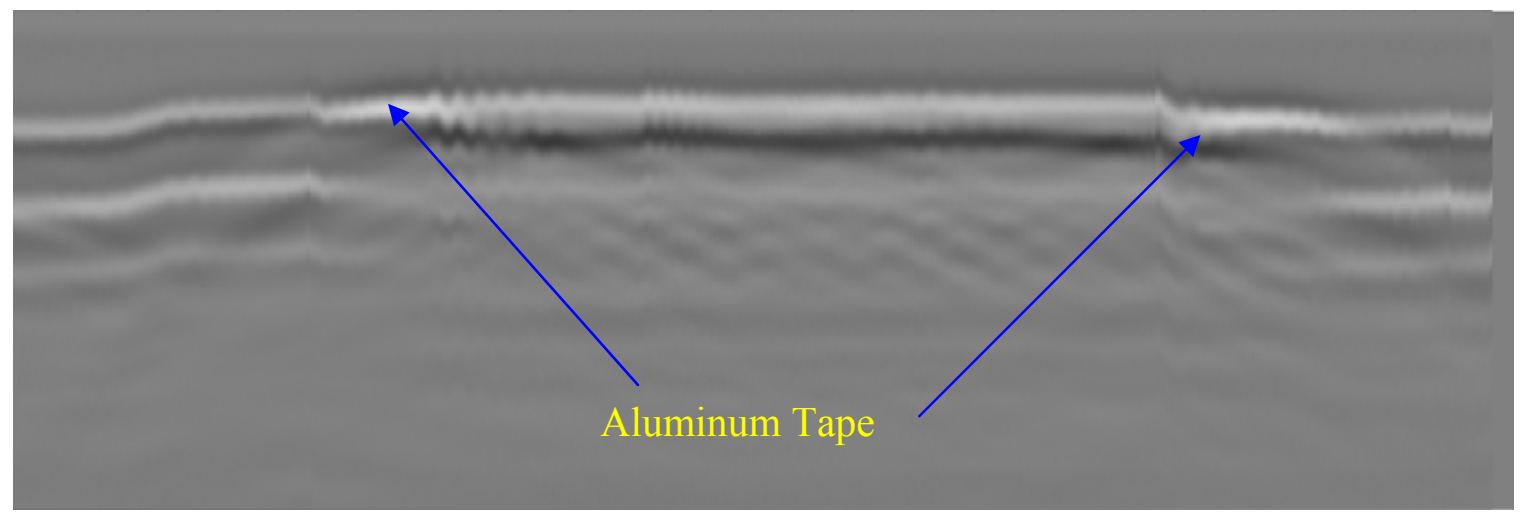

Figure 7.9: A typical GPR scan of an FRP deck using 2.0GHz air-coupled horn antenna.

In this experiment, three passes of GPR scanning were conducted along the length of the FRP deck. Each length pass included a defect-free wearing surface module followed by a defective wearing surface module (with defect attached to the bottom side of the wearing surface, thus simulating debonds) and then another defect-free module. By collecting multiple (three in this case) GPR passes for the test deck at equal distances (9" or $228.6 \mathrm{~mm}$ ) apart, RADAN post-processing software enabled the creation of a 3D profile of the scanning project (Figure 7.10). This 3D profile (obtained using groundcoupled antenna in this case) is very useful as it allows user to visually identify any detectable defects and pinpoint its exact location through the 3D coordinate system. Most 3D data have to be gained (amplified) during the post-processing stage in order to identify smaller defects. 


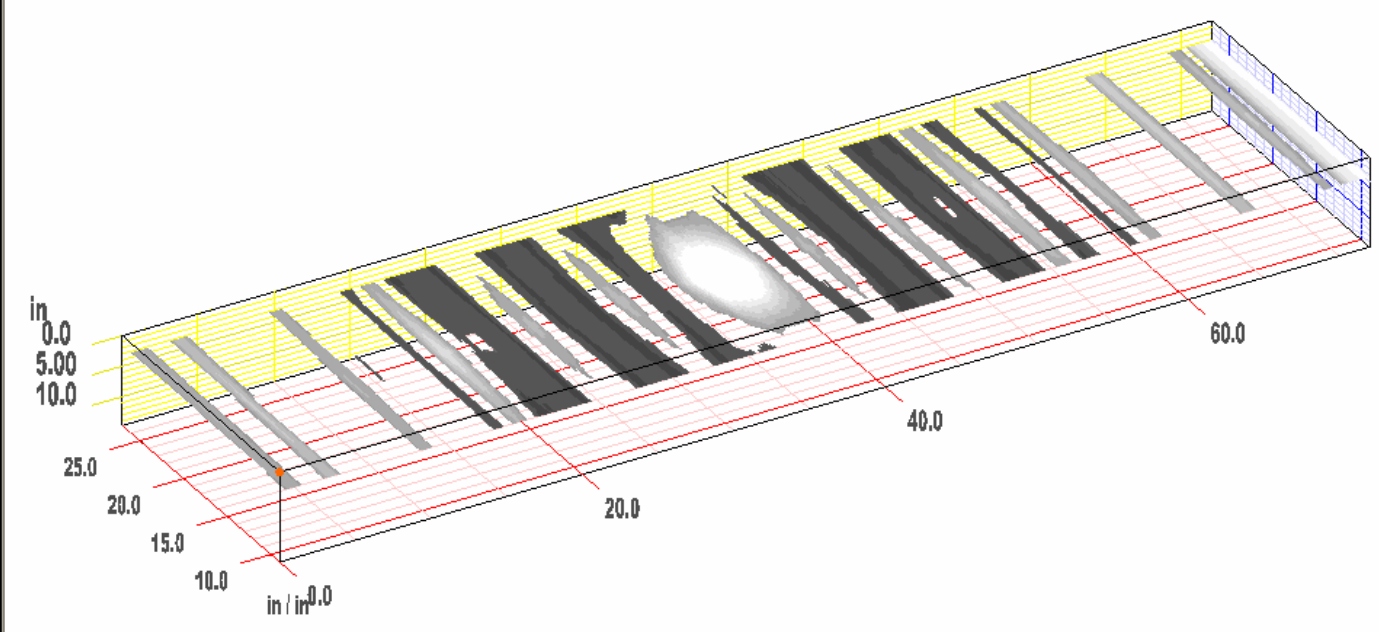

Figure 7.10: 3D profile created by collecting three passes of GPR data at equal distances (9") apart using ground-coupled antenna.

There are several few post-processing options available through the use of the post-processing software, RADAN. Process such as zero-correction, will remove the gap between the antenna and the top surface of the first medium. This process essentially enables the user to identify the depths of detectable defects. Another one of the many post-processing options available is the Finite Impulse Response (FIR) filter. FIR filtering removes any constant system noise generated within the GPR system. For this experiment, the FIR filter was applied to all data sets. The data were then gained in order to identify any subsurface defects. Figure 7.11 shows the GPR scan after the FIR was applied to the original (raw) data set shown in Figure 7.8 (ground-coupled antenna). It should be noticed that the uniform "top" layer that is visible in Figure 7.8 no longer exists after the FIR was applied (Figure 7.11). Also, the application of gain has made the features in the GPR scan look much sharper in Figure 7.11. 


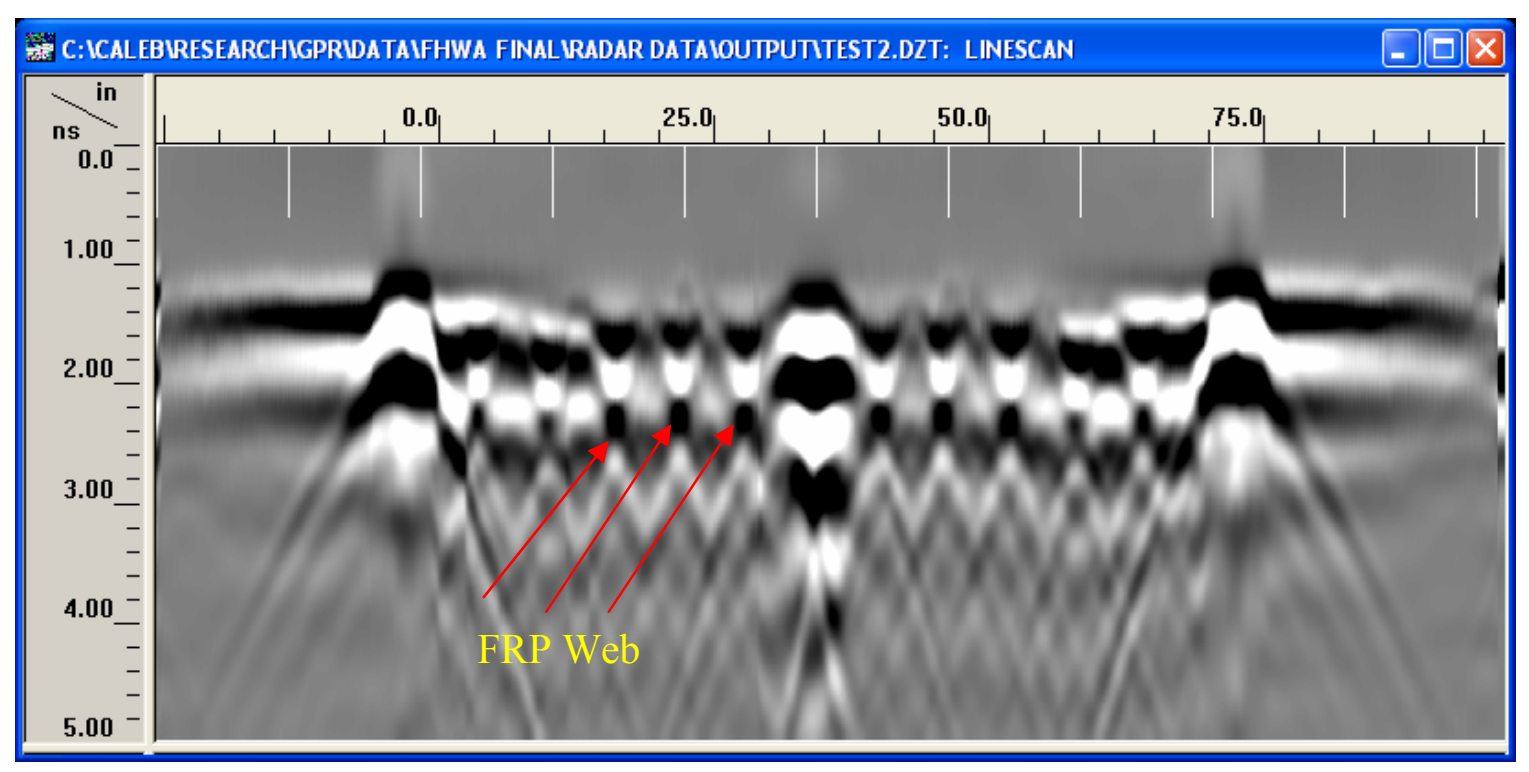

Figure 7.11: GPR processed data after FIR filter was applied to the raw data in Figure 7.8.

Large water-filled defect (4" x 4" x $0.09 "$ or $101.6 \mathrm{~mm}$ x $101.6 \mathrm{~mm} \times 2.25 \mathrm{~mm})$ could be easily detected by using a $1.5 \mathrm{GHz}$ ground-coupled antenna (Figure 7.12 ). The bright spot in the middle of the deck is due to the presence of the water-filled debond. The predicted location was later confirmed by measurement.

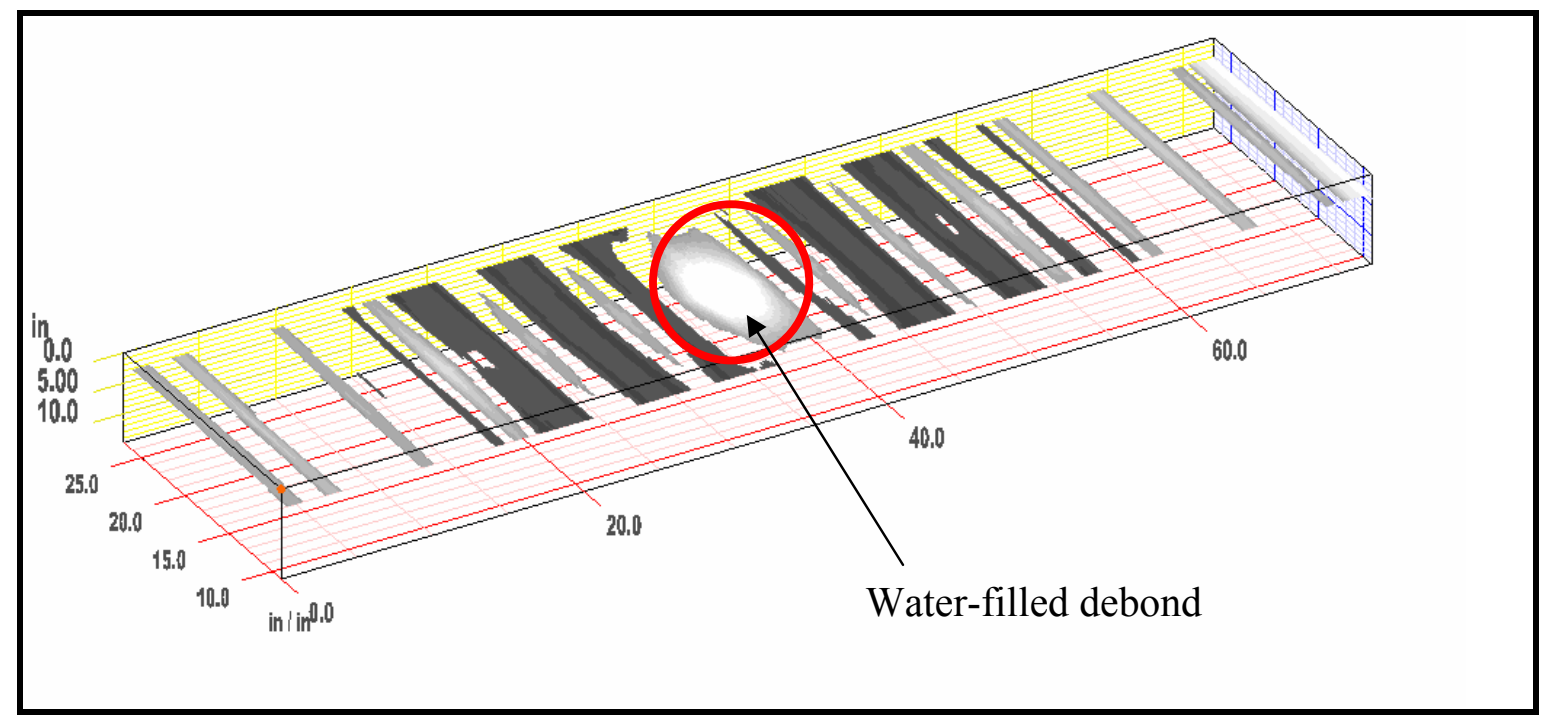

Figure 7.12: 4" x 4" x 0.09" (101.6mm x 101.6mm x 2.25mm) water-filled debond shown in 3D profile using ground-coupled antenna. 
A 4" x 4" x 0.09" (101.6mm x 101.6mm x 2.25mm) air-filled defect could not be detected using the 3D GPR profile (Figure 7.13), which was found to be very noisy.

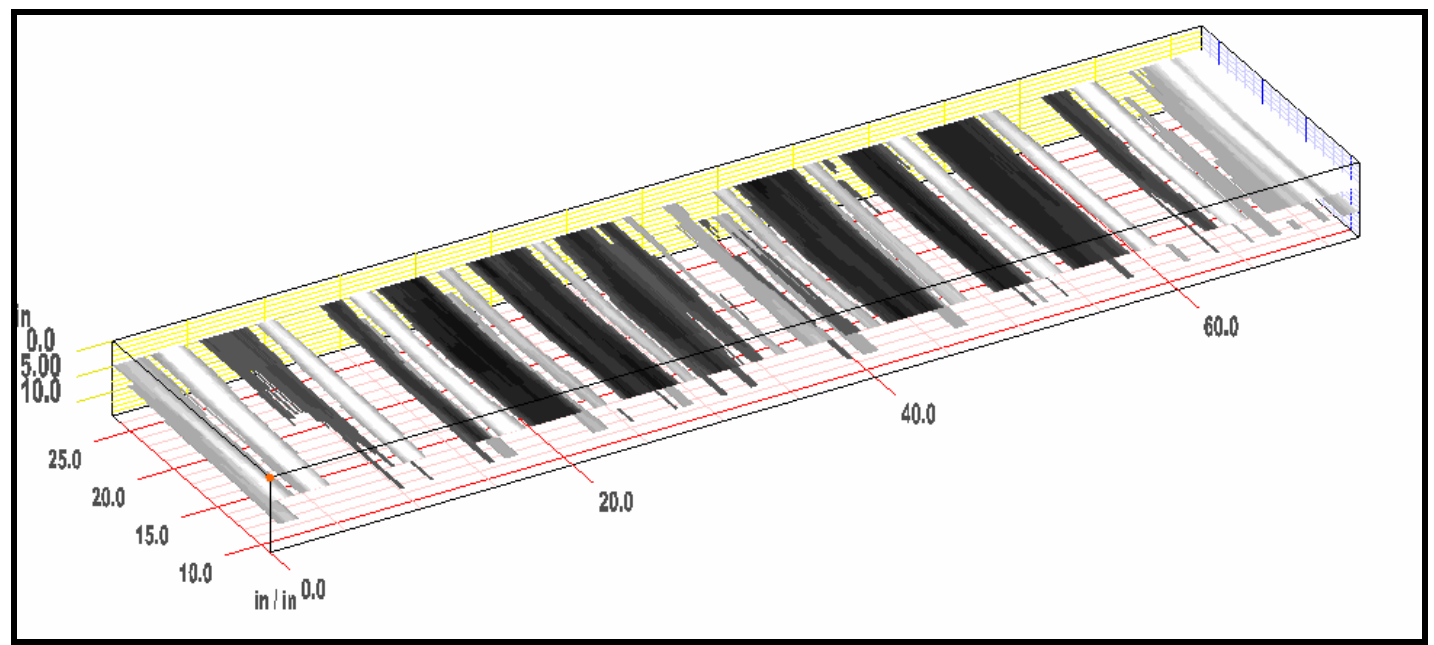

Figure 7.13: 4" x 4" x 0.09" (101.6mm x 101.6mm x 2.25mm) air-filled debond in the central region of the FRP deck is not visible in this $3 \mathrm{D}$ profile obtained using ground-coupled antenna.

3D profiles of every data set (with different configuration of subsurface defects) were generated. It was noted that the ground-coupled antenna's data produced a clearer and more useful 3D profiles (e.g., Figure 7.12) while the horn antenna profile (Figure 7.14) indicated a high degree of wave attenuation.

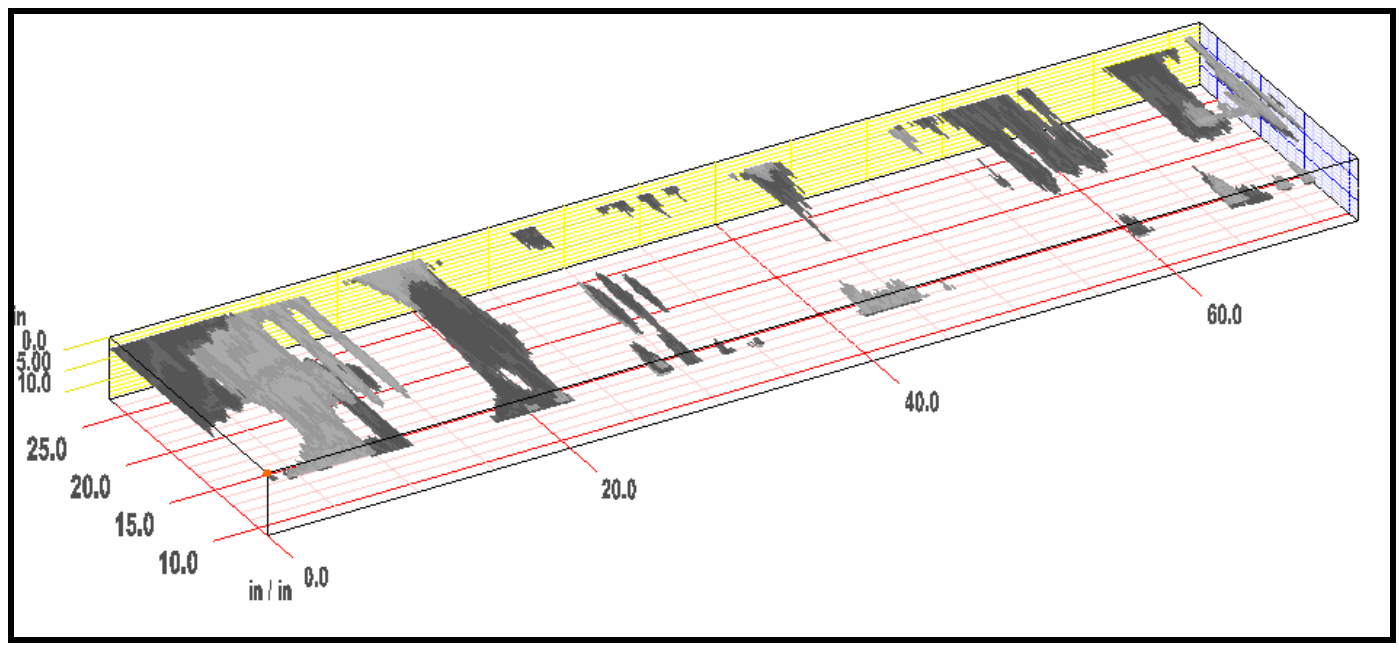

Figure 7.14: 3D profile generated by the air-coupled horn antenna. 
As shown in Figure 7.14, the features were sparse and not uniform. The features only show noise, and one cannot make a firm conclusion based on the 3D profile obtained using the air-coupled antenna. The ground-coupled antenna's 3D profile could easily detect subsurface water-filled debonds (Figure 7.12) of size 4" x 4" x 0.09 " $(101.6 \mathrm{~mm} \times 101.6 \mathrm{~mm} \times 2.25 \mathrm{~mm})$ and greater.

While 3D profile offers a user friendly way to identify subsurface defects, there are many instances where inspecting and even processing the 2D scan profile could yield better results. For instance, the amplified FIR scan of 4" x 4" x 0.03 " $(101.6 \mathrm{~mm} \times$ $101.6 \mathrm{~mm} \times 0.75 \mathrm{~mm}$ ) water-filled defect could be identified through the $2 \mathrm{D}$ profile (Figure 7.15) but not via the 3D profile. By visual inspection of Figure 7.15, one can conclude that the middle portion of the scan is unusual. Since the aluminum tapes can easily be spotted towards the beginning and the end of the 2D scan, the distance of the "unusual" spot to one of the aluminum tapes could be measured. This measurement confirmed that the unusual spot was indeed the 4" x 4" x 0.03 " $(101.6 \mathrm{~mm}$ x $101.6 \mathrm{~mm} x$ $0.75 \mathrm{~mm}$ ) water-filled debond.

Another characteristic of the water-filled debond is that it tends to create an upward "wave". The same upward wave is very obvious in the middle region of the scan in Figure 7.11, which is once again the 4" $\mathrm{x}$ 4" $\mathrm{x} 0.09$ " $(101.6 \mathrm{~mm} \times 101.6 \mathrm{~mm} \times 2.25 \mathrm{~mm})$ water-filled defect. By inspecting the 2D scan, the smallest defect that could be detected was 2" x 2" x 0.06" (50.8mm x 50.8mm x 1.5mm) water-filled debond (Figure 7.16). By knowing the characteristic of a water-filled debond (upward "wave") as well as obvious visual indications, GPR proved to be an extremely useful tool in detecting subsurface water-filled debonds between the wearing surface and the underlying FRP bridge deck. 


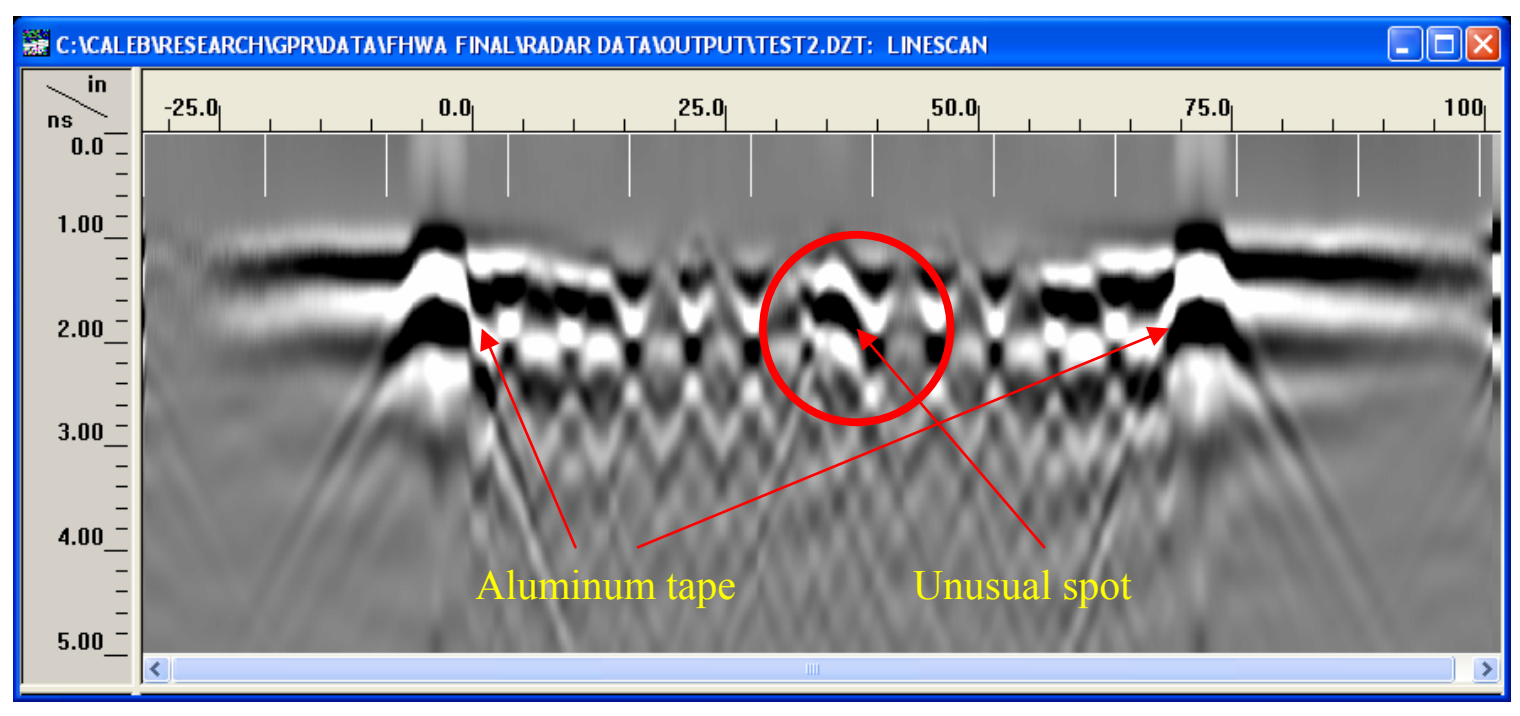

Figure 7.15: 4" x 4" x 0.03" (101.6mm x 101.6mm x 0.75mm) water-filled debond seen in a 2D GPR scan obtained using ground-coupled antenna.

Unfortunately, the air-filled defects were not visible in the 2D or 3D GPR profiles due to the fact that the dielectric constant of air is relatively close to FRP. In the case of water-filled defect, the complex dielectric permittivity of water is significantly higher compared to the FRP which makes the amplitude of the water-filled debond to be significantly higher. While visual inspection of the 2D and 3D plot is an acceptable tool for detecting subsurface defects, a more systematic approach such as automated algorithms is needed for future advancement of the inspection system. Besides, it is a good practice to verify the validity of the visual inspection of the $2 \mathrm{D}$ and $3 \mathrm{D}$ profiles by other means. To achieve these goals, the individual scans at the location of the defects and at a defect-free area were identified and converted into ASCII format. These ASCII files were then imported into Excel spreadsheet. Simple analysis was done to confirm the validity of visual inspection of $2 \mathrm{D}$ and $3 \mathrm{D}$ profiles. 


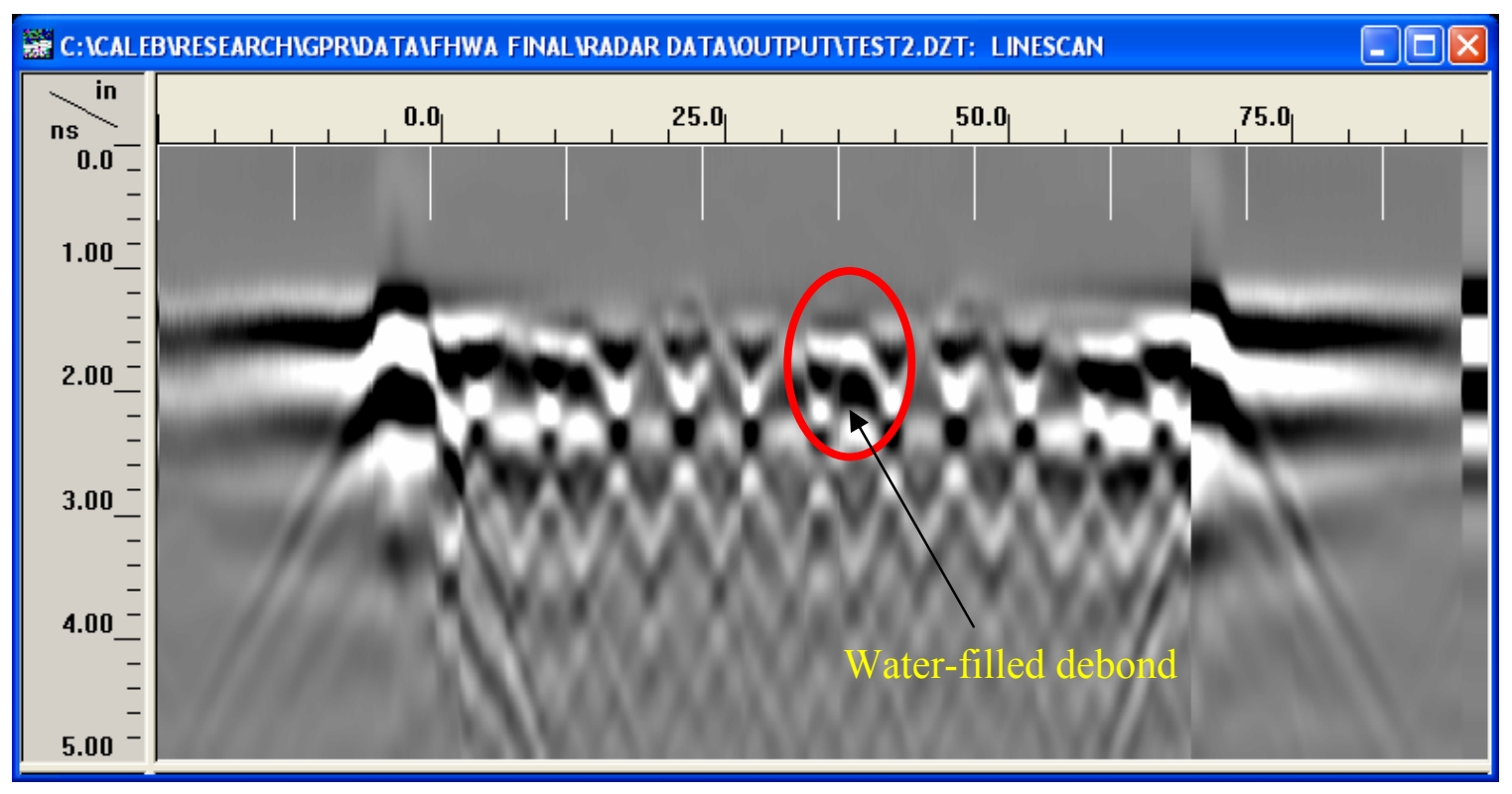

Figure 7.16: 2" x 2" x 0.06" (50.8mm x 50.8mm x 1.5mm) water-filled debond.

A 5 to 6 character code system was used to help analyze this set of data. The first two characters represent the size of the subsurface debond in inches. The third character represents the thickness of the defect ( 1 for 0.03 " or $0.75 \mathrm{~mm}$ thick, 2 for 0.06 " or $1.5 \mathrm{~mm}$ thick, and 3 for 0.09 " or $2.25 \mathrm{~mm}$ thick). The fourth character was a "W" for water-filled defects or an "A" for air-filled defects. The last one or two character would be "GC" which stands for ground-coupled antenna, or " $\mathrm{H}$ " which stands for horn antenna. For example, a “443WGC” means a 4" x 4" x 0.09” (101.6mm x 101.6mm x 2.25mm) waterfilled debond scan with a $1.5 \mathrm{GHz}$ ground-coupled antenna. "DFGC" is used occasionally to represent the defect free waveform collected using the ground-coupled antenna.

As indicated in Figure 7.17, the 443WCG, i.e., 4" x 4" x 0.09" (101.6mm x $101.6 \mathrm{~mm} \times 2.25 \mathrm{~mm}$ ) water-filled debond can be easily detected because of its higher than usual amplitude compared to the defect-free waveform. Notice that the $223 \mathrm{WGC}$, or 2 " x 2" x $0.09 "(50.8 \mathrm{~mm} \times 50.8 \mathrm{~mm} \times 2.25 \mathrm{~mm})$ water-filled debond has a comparable, but 
smaller amplitude waveform. Though the amplitude is smaller than the 443WGC case (yet slightly higher than the defect free waveform), the $223 \mathrm{WGC}$ is obviously a waterfilled defect because its phase is similar to 443WGC. Also notice that the 222WGC waveform, which corresponds to a 2 " $\mathrm{x} 2$ " x 0.06 " $(50.8 \mathrm{~mm} \times 50.8 \mathrm{~mm} \times 1.5 \mathrm{~mm})$ waterfilled debond, is showing a slight time-shift. The waveforms corresponding to the 0.03 " $(0.75 \mathrm{~mm})$ thick water-filled debonds (221WGC and 441WGC) are more or less aligned with the defect-free waveform. While it is clear that the signal amplitudes in these waveforms (221WGC and 441WGC) are slightly higher than the defect-free waveform (DFGC), such distinction may not be obvious in the field data. Thus, it would be difficult to detect $0.03 "(0.75 \mathrm{~mm})$ thick water-filled debonds, while water-filled debonds with larger thicknesses $(\geq 0.06$ " or $1.5 \mathrm{~mm})$ could be detected.

To further investigate this result, signal subtraction was carried out. The defectfree signal was subtracted from the signals corresponding to the various water-filled debonds. Figure 7.18 shows the waveforms for the various water-filled debonds after the subtraction was carried out. 


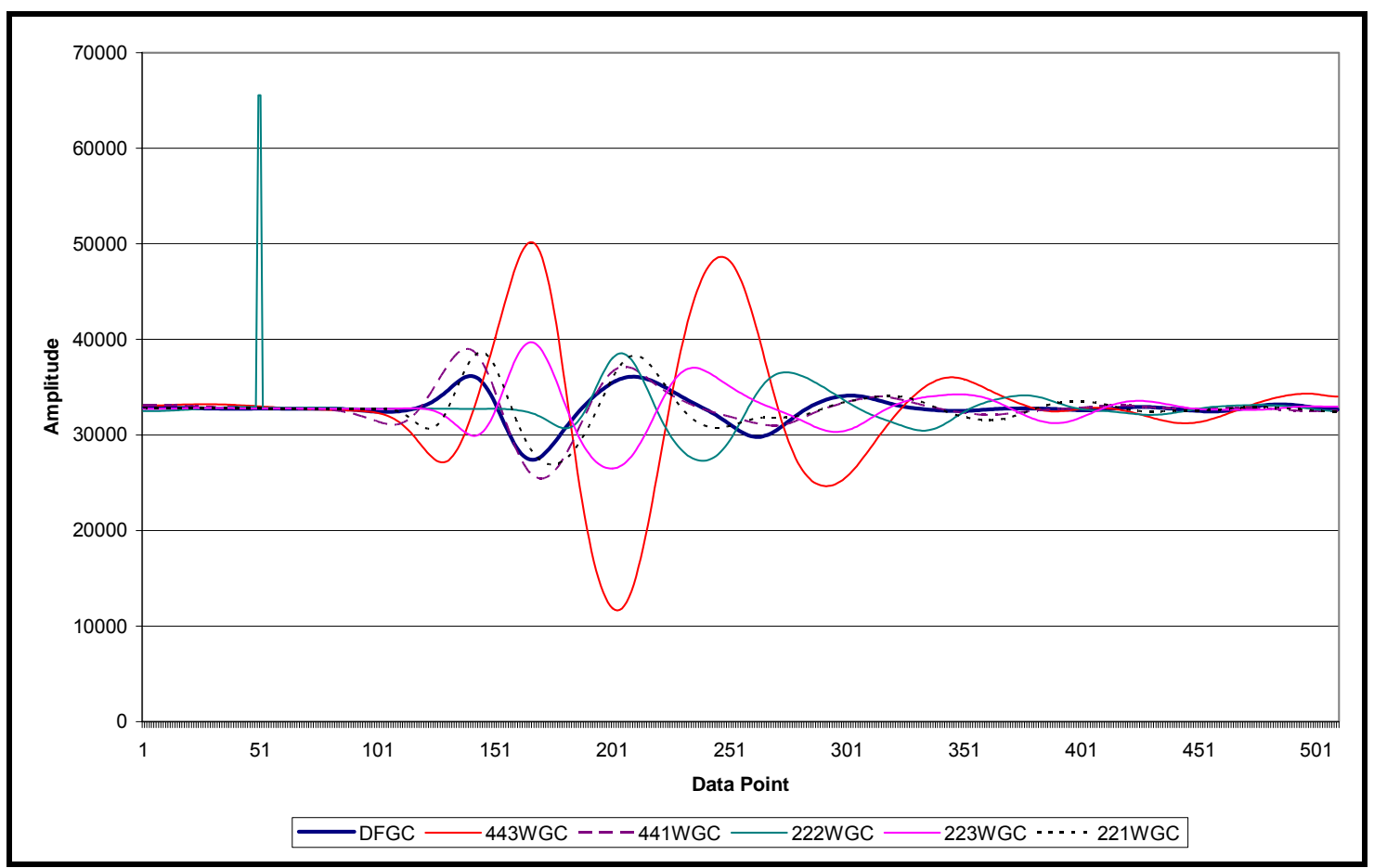

Figure 7.17: Comparison of signal amplitudes for GPR waveforms (ground-coupled antenna) from various water-filled debonds.

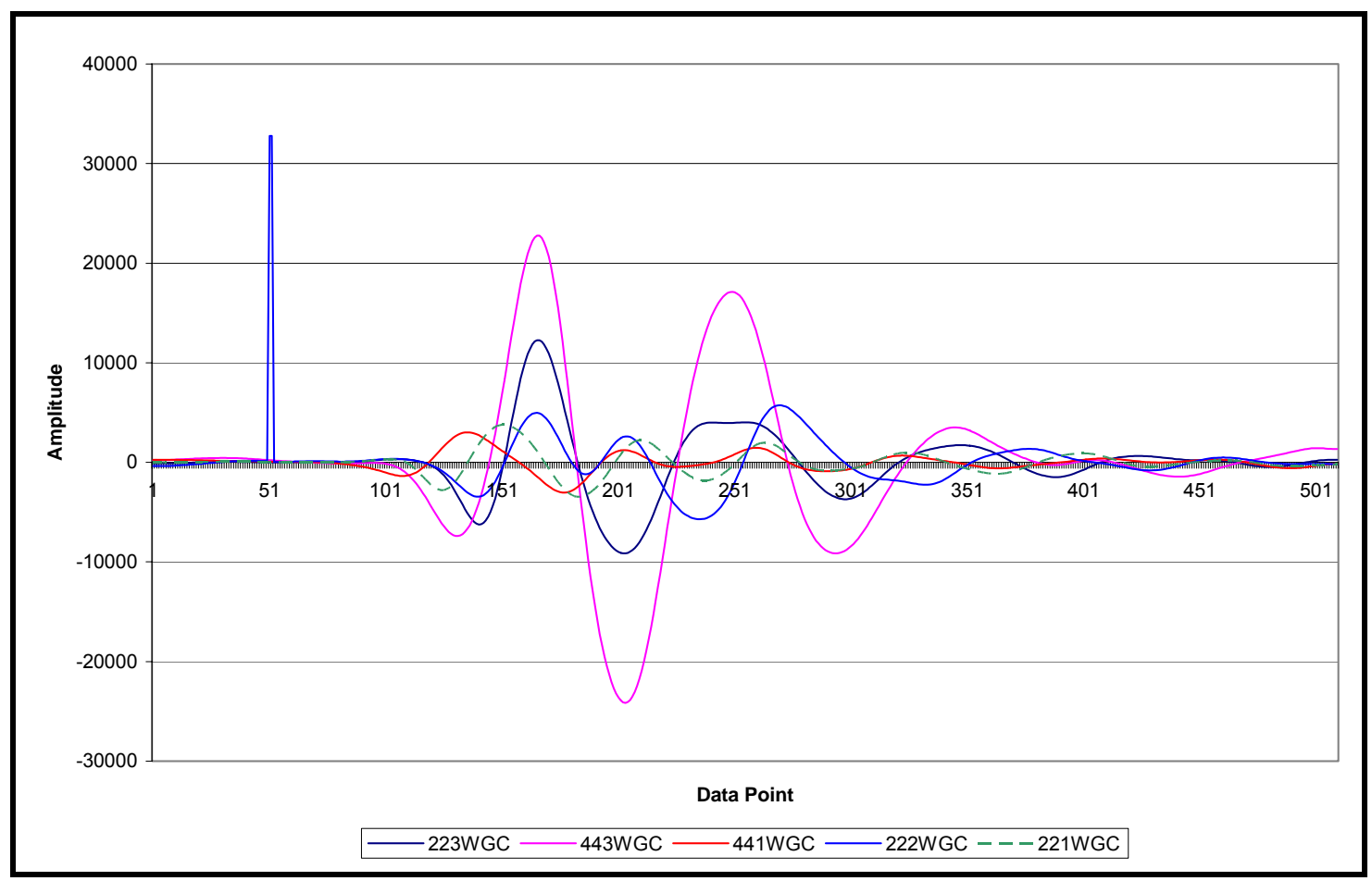

Figure 7.18: Waveforms corresponding to various debonds after subtraction of defect-free waveform (ground-coupled antenna). 
As seen from Figure 7.18, 223WGC and 443WGC show higher amplitudes while the other waveforms show significantly lower amplitudes. Though the laboratory setting allows the smaller debonds to be detected through such GPR waveform comparisons, the amplitude of such debonds are not significant enough to confidently classify as defect, especially in the field setting when many other factors could give rise to signals of comparable amplitude.

A similar analysis was conducted for the GPR waveform from subsurface debonds obtained using air-coupled horn antenna. Unfortunately, as seen in Figure 7.19, the horn antenna waveforms yield no useful information. Also, the spikes in Figure 7.19 are the result of noises introduced during the processing of GPR data.

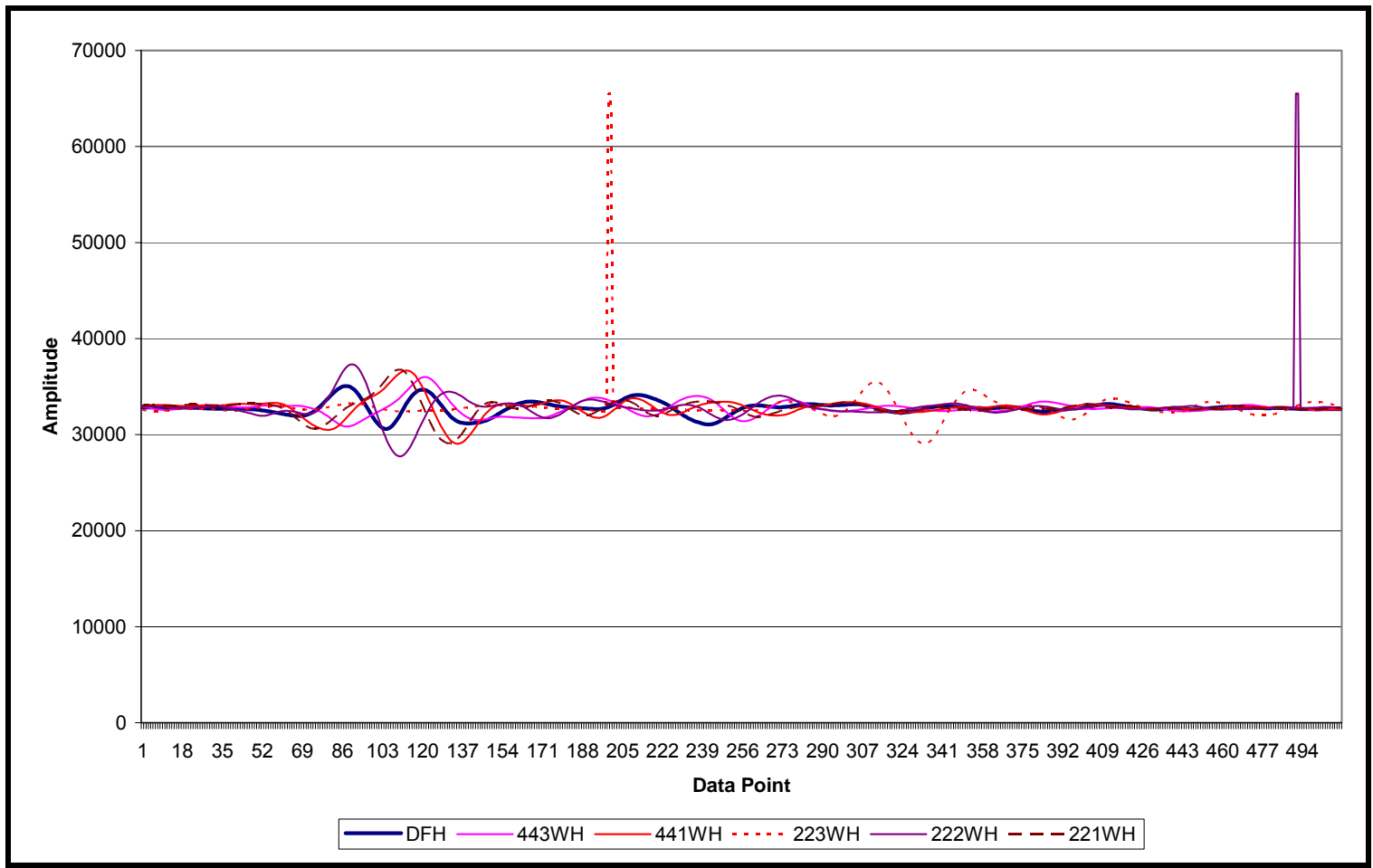

Figure 7.19: Comparison of signal amplitudes for GPR waveforms (air-coupled horn antenna) from various water-filled debonds. 
In lieu of previous results which indicated the inability of $2.0 \mathrm{GHz}$ air-coupled antenna GPR system to detect smaller defects (2" x 2", 4" x 4" defects with thickness varies from 0.03 " to $0.09 ")$, various larger size defects, such as 8 " 8 " $203.2 \mathrm{~mm})$ and $12 " \times 12 "(304.8 \mathrm{~mm} \times 304.8 \mathrm{~mm})$ debond, were created to accurately identify the minimum size and thickness defect detectable by the $2.0 \mathrm{GHz}$ air-coupled antenna GPR system. The defects' thicknesses ranged from 0.03 " $(0.75 \mathrm{~mm})$ to 0.09 " $(2.25 \mathrm{~mm})$. The layout of the defects is shown in Figure 7.20.

Data were collected by using the $2.0 \mathrm{GHz}$ horn antenna GPR system on the modules with air-filled defects follow by water-filled defects. As with any other GPR procedure, a set of fixed parameters were established to ensure uniformity of the data collected. In this case, the gain during the data acquisition was set to 10 times, while lowering the range down to $5 \mathrm{~ns}$ with 1024 points collected with each pass.

The data were then analyzed by using the Radan 6.0 software. Finite Impulse Response (FIR) was applied to all the data, and a 3D model was created as well. As noted in the previous reports, the key to analyzing GPR data is to look at a combination of the 2D image, 3D image, and the signal analysis in order to draw conclusions.

Results show that the $2.0 \mathrm{GHz}$ horn antenna GPR system is not capable of detecting subsurface air-filled defects (Figure 7.21). As such, the next logical step is to apply the FIR in hope of eliminating the constant signal noise thus improving the quality of the 2D plot. Unfortunately, the result of FIR did not reveal any helpful result either. Finally, the files were stitched together to form a 3D image. As with the procedures before, it too did not reveal any conclusive result. By knowing the exact location of the 
defects beforehand, 10 scans of data were extracted from the location of each defect and converted into ASCII format for signal analysis. The average of the 10 scans was used.

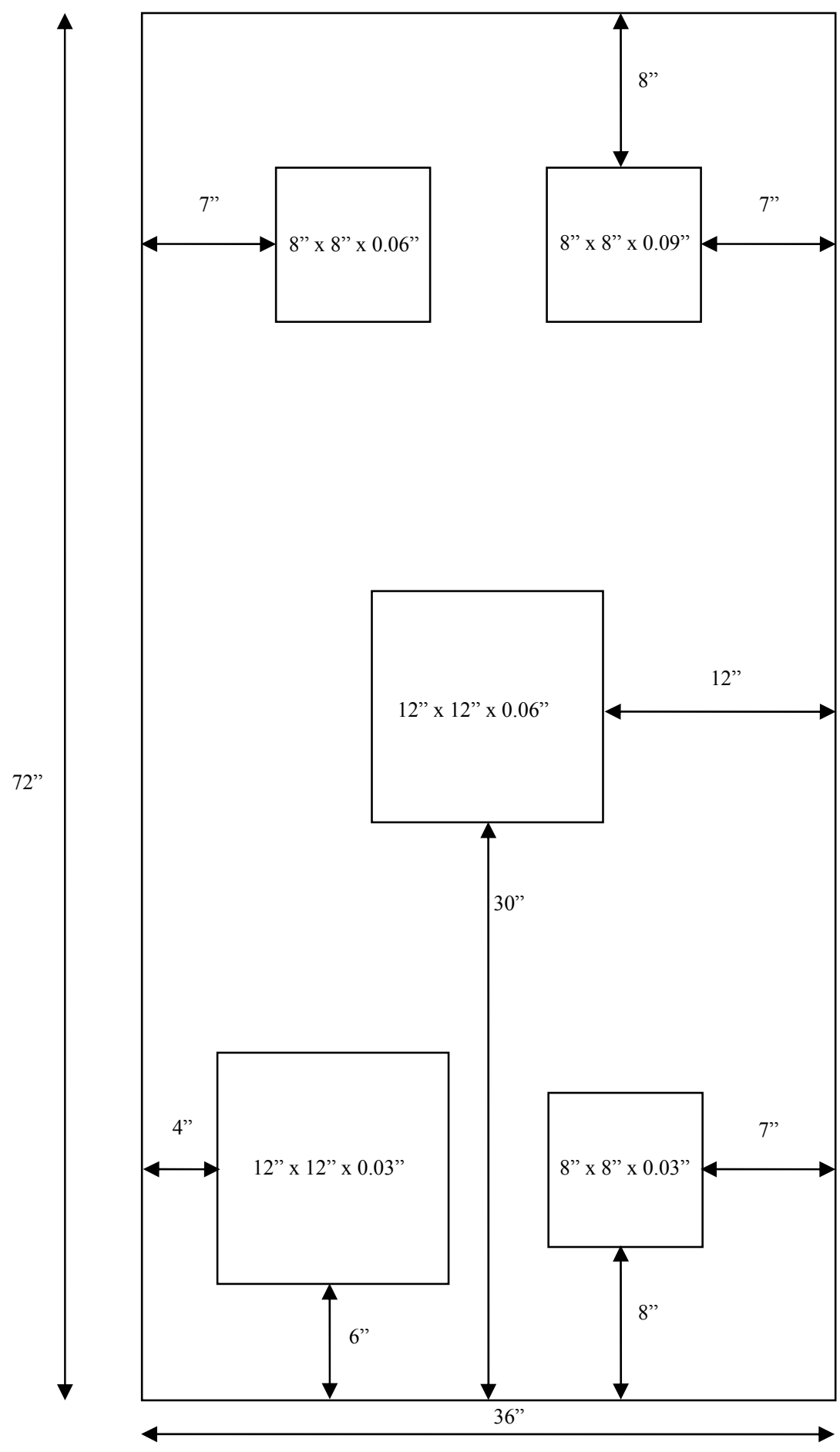

Figure 7.20: Layout of defects. 


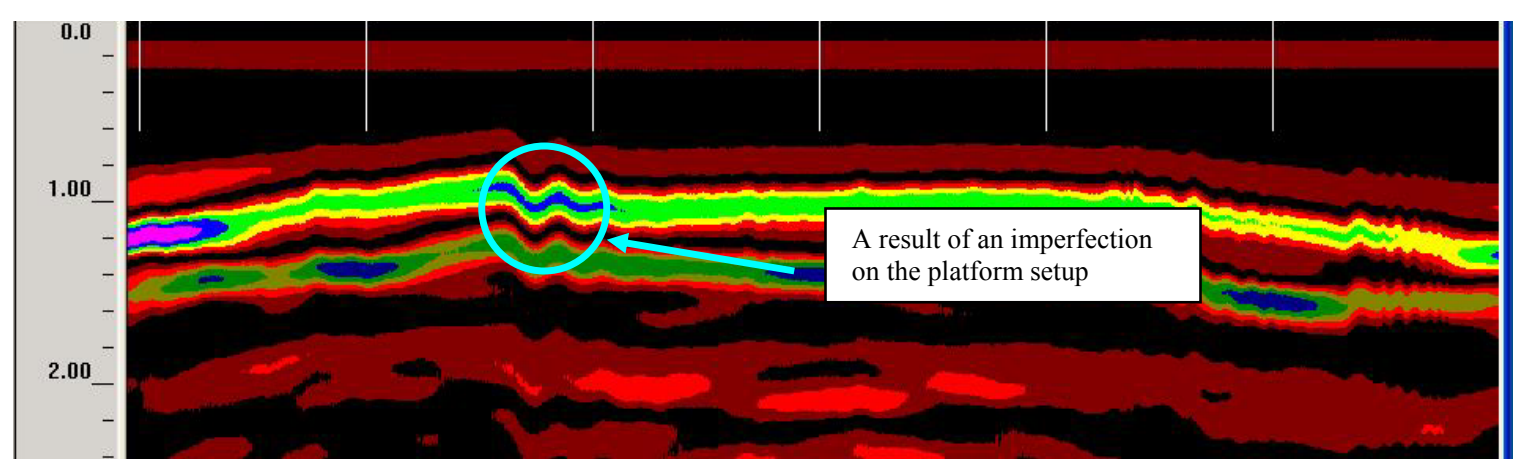

Figure 7.21: 2D image of an air-filled debond scan.

Figure 7.22 shows the GPR signals corresponding to the air-filled defects data prior to applying FIR. Each waveform corresponds to various air-filled debonds after subtraction of defect-free waveform. The maximum amplitude of each waveform is about 4000 points within each other, hovering around the 6000 point mark. Compared to the same signal analysis for a ground-coupled antenna (Figure 7.23), the signal amplitudes (after subtraction of defect-free waveform) for the air-filled defects clearly is not significant enough to show any promising results (note that all analysis figures use a 70,000 point scale for easy comparison). In hopes of getting better responses, a FIR analysis was done to each set of data, prior to taking the average value. Once again, it did not reveal any compelling results, since the resulting signals had comparable signal strengths as the original data sets (Figures 7.24 and 7.22). 


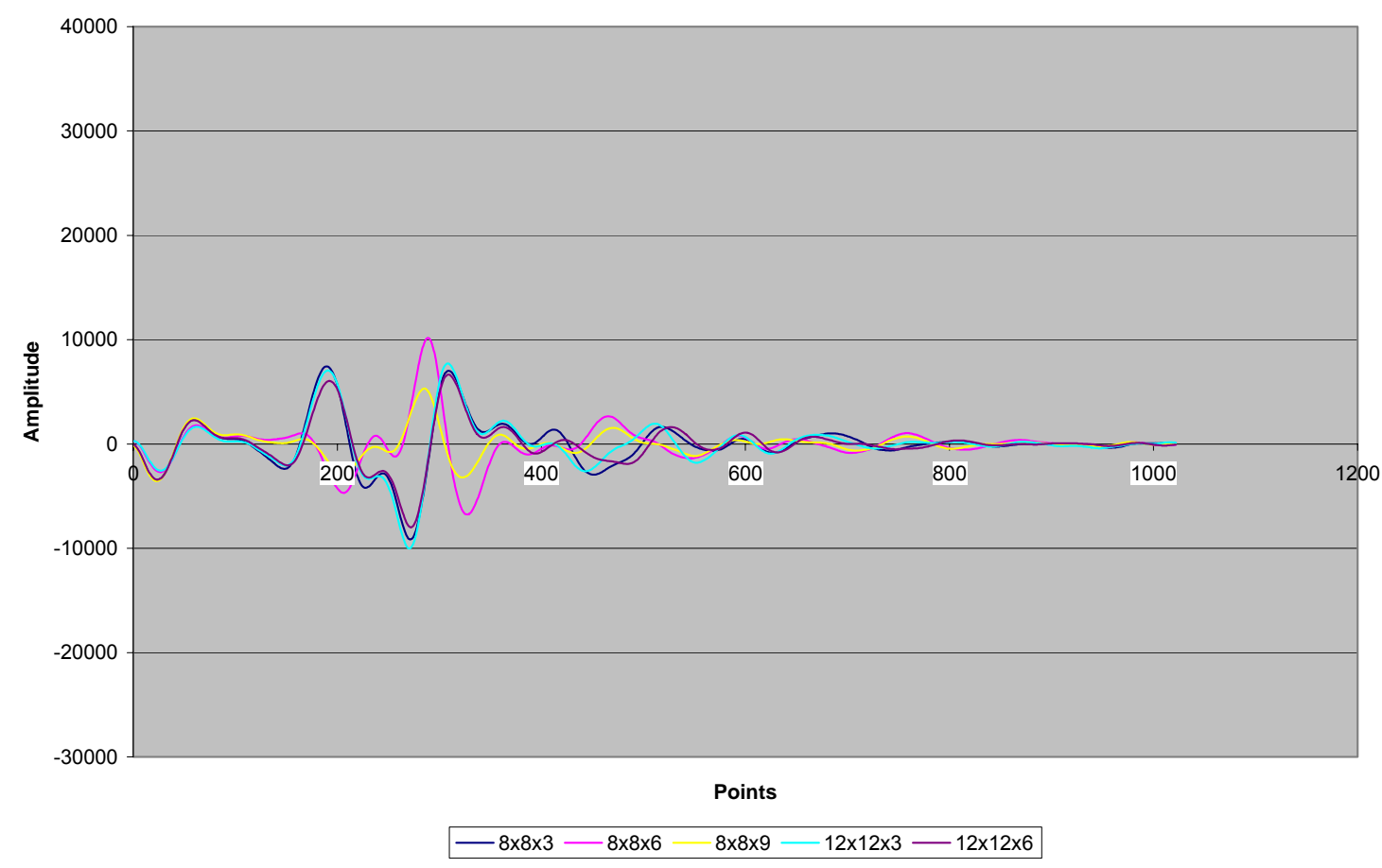

Figure 7.22: Signals corresponding to the original air-filled defects data after subtraction of defectfree waveform (horn antenna).

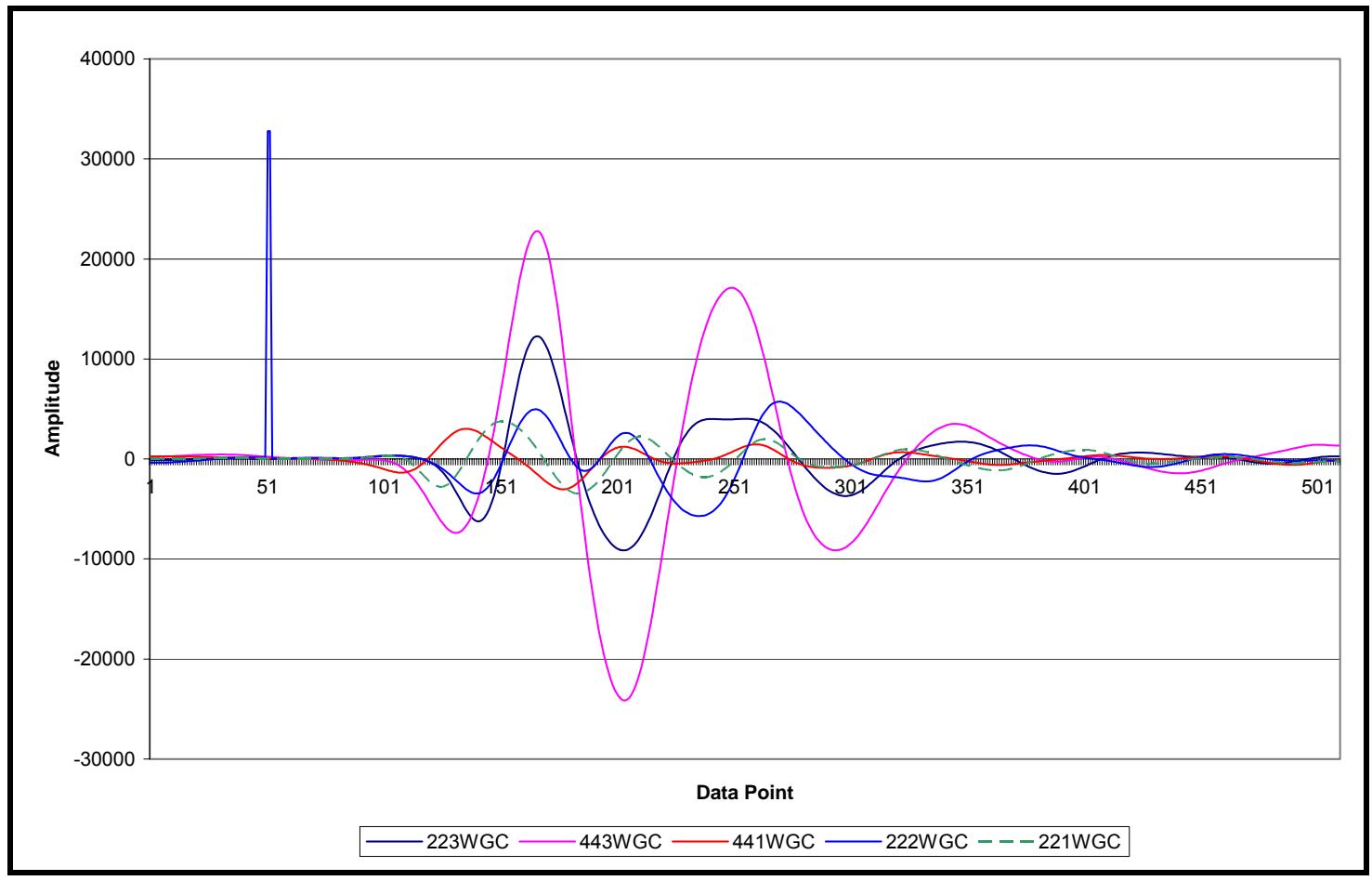

Figure 7.23: Waveforms corresponding to various water-filled debonds after subtraction of defect-free waveform (ground-coupled antenna). 


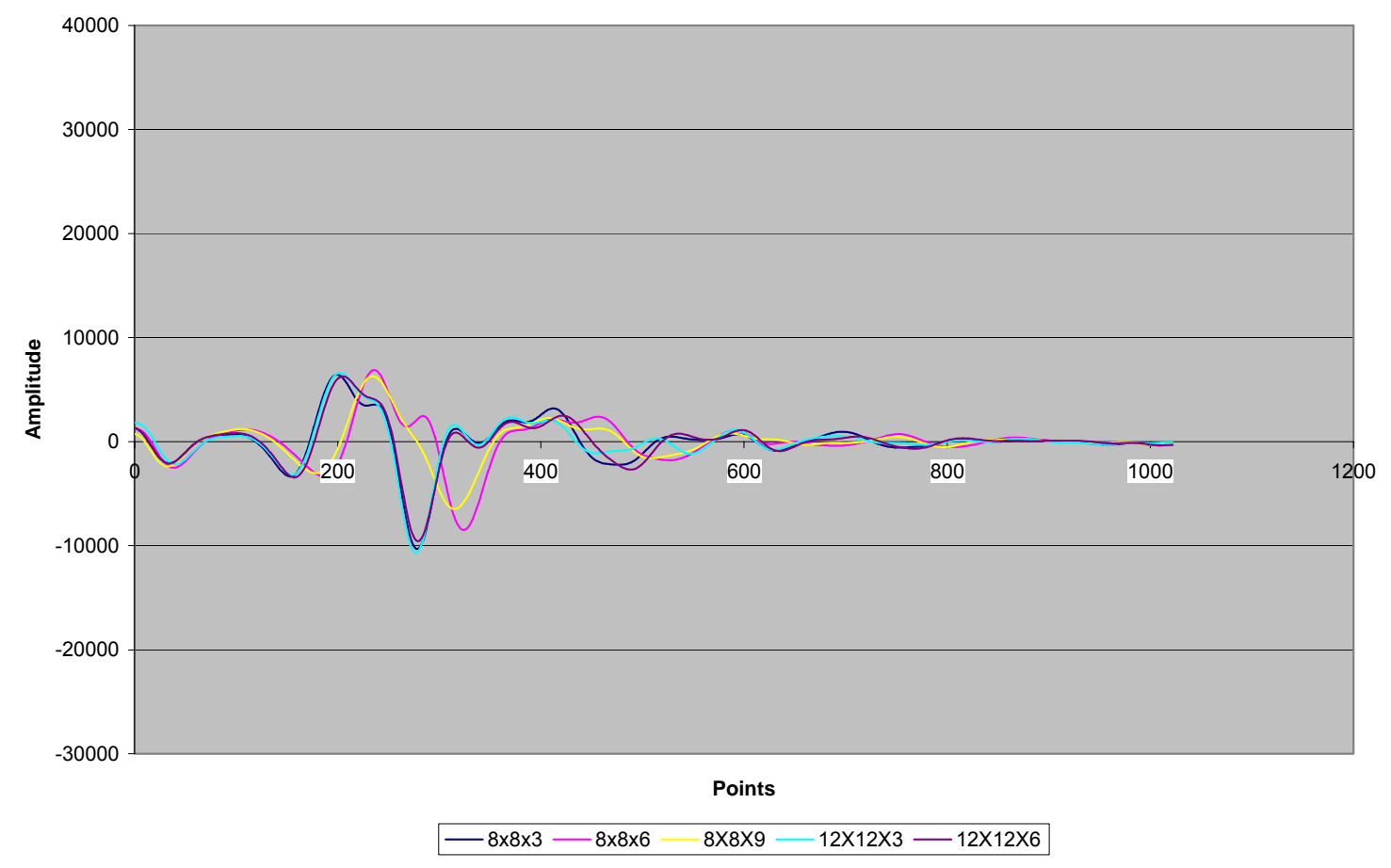

Figure 7.24: Signals corresponding to the air-filled defects data, after subtraction of defect-free waveform, with FIR (horn antenna).

Quick glance at the 2D image of a water-filled defect scan (horn antenna) showed that it is very promising. As easily seen in Figure 7.25, there is a questionable spot in the $2 \mathrm{D}$ image. Note that this is the scan of the deck portion with a 12 " $\times 12$ " $\times 0.03$ " waterfilled defect at 12 " from the beginning of the scan and another 8 " x 8 " x 0.06 " waterfilled defect at 12" from the end of the scan. However, it is not conclusive enough to draw a conclusion based on only one 2D image. Hence, FIR filter was applied to the original set of data (Figure 7.26).

Interestingly, the defect that was first identified by the 2D image has disappeared. However, the FIR image (Figure 7.26) did show the second water-filled defect, which has a smaller plan size but larger thickness at 0.06 ". Figures 7.27 and 7.28 show the 2D image of the original and FIR scan for the 12" x 12" x 0.06 " water-filled defect. Likewise, Figures 7.29 and 7.30 show the 2D image of the original and FIR scan for the 
8 " 8 " 80.03 " water-filled defect at 8 " from the beginning of the scan and 8 " 8 " $\mathrm{x}$ 0.09 " water-filled defect at 8 " from the end of the scan. Figures 7.27 and 7.28 clearly show the 12 " x 12 " x 0.06 " water-filled defect. Figure 7.29 did not reveal anything while Figure 7.30 clearly shows the 8 " x 8" x 0.09 " water-filled defect.

To further evaluate the data, a 3D scan was constructed (Figure 7.31). As usual, a FIR version of the 3D scan was constructed as well for comparison purposes (Figure 7.32).

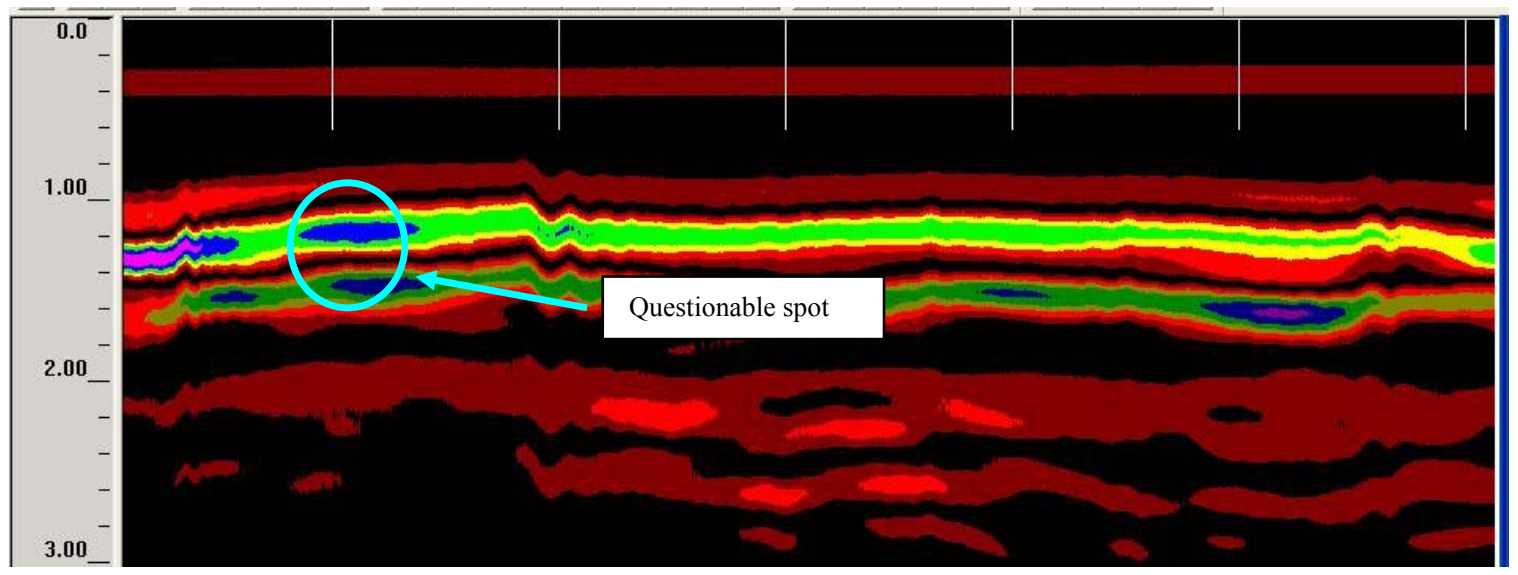

Figure 7.25: 2D image of a water-filled debond scan.

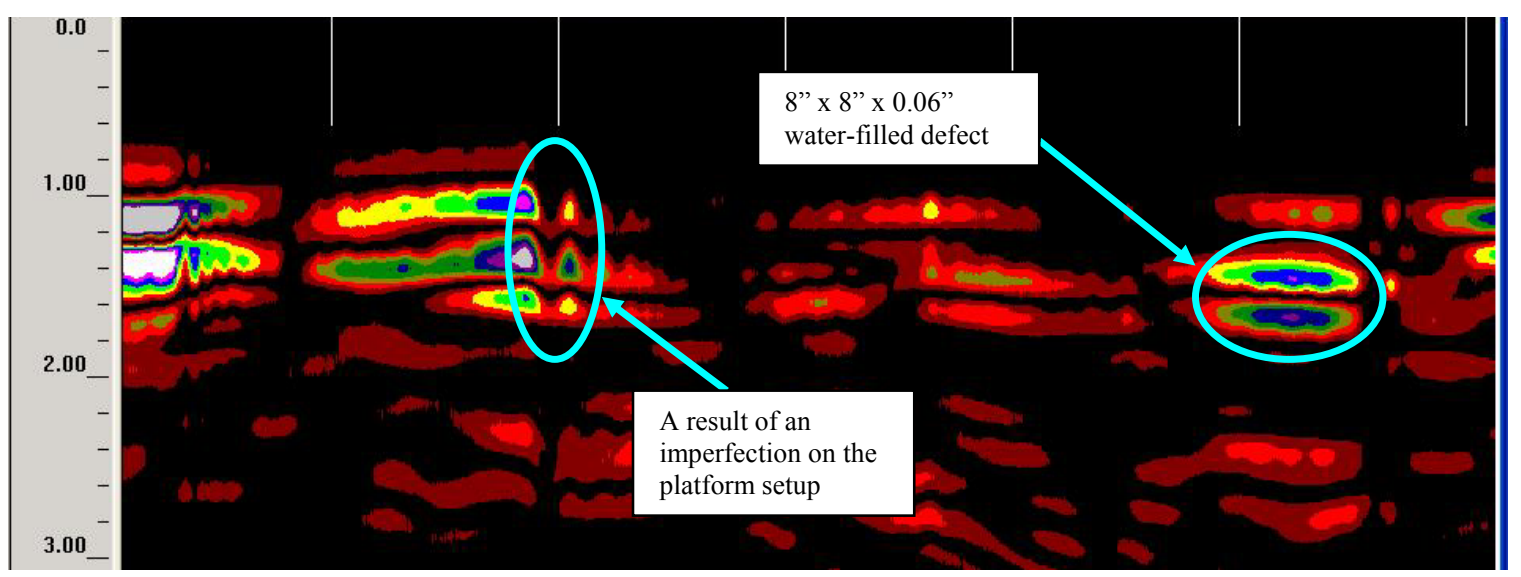

Figure 7.26: 2D image of a water-filled debond scan with FIR. 


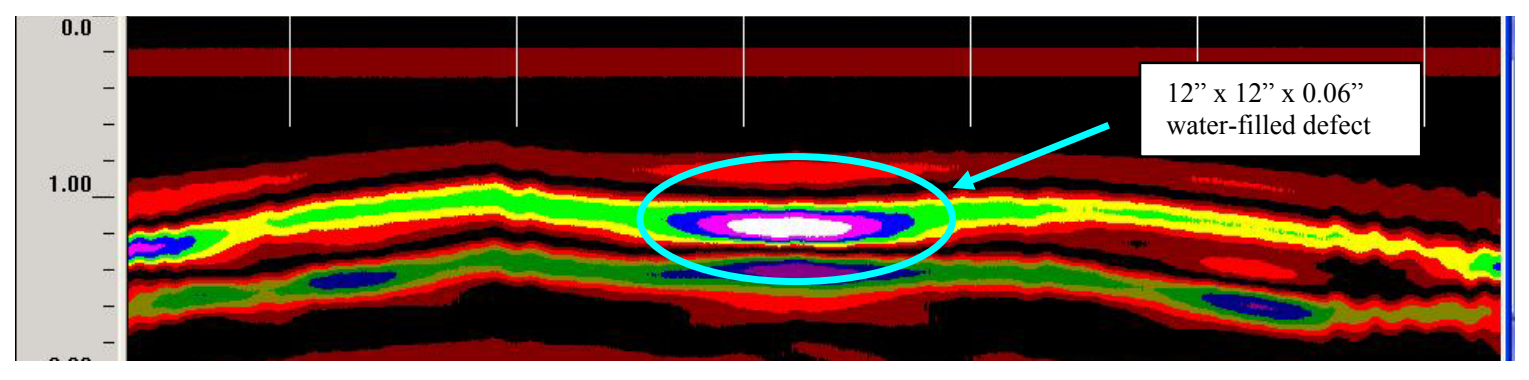

Figure 7.27: 2D image of the original scan for the 12 " $\mathrm{x} 12$ " $\mathrm{x} 0.06$ " water-filled defect.

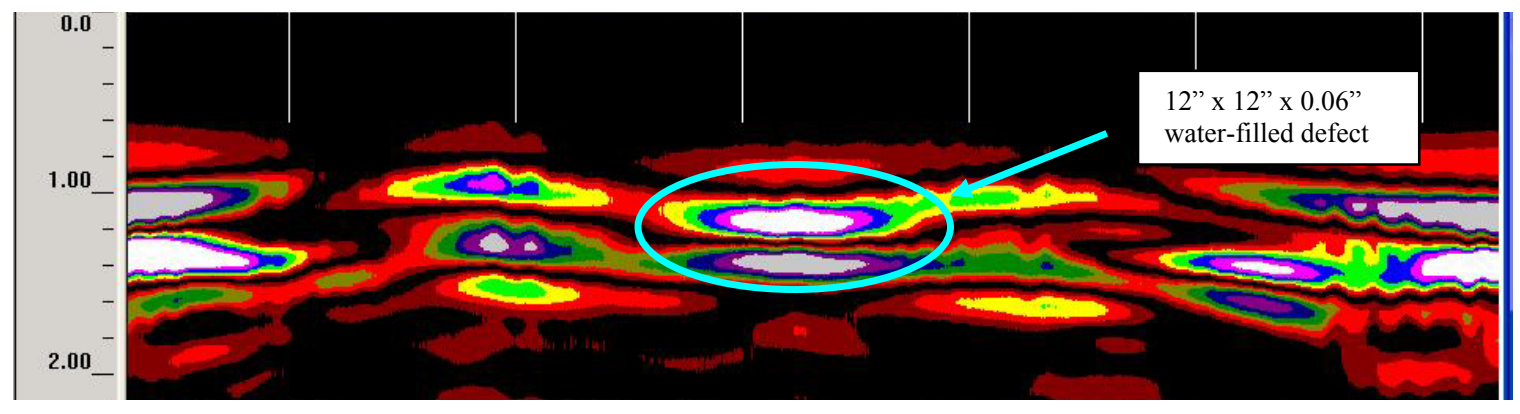

Figure 7.28: 2D image of the FIR scan for the 12 " x 12 " x 0.06 " water-filled defect.

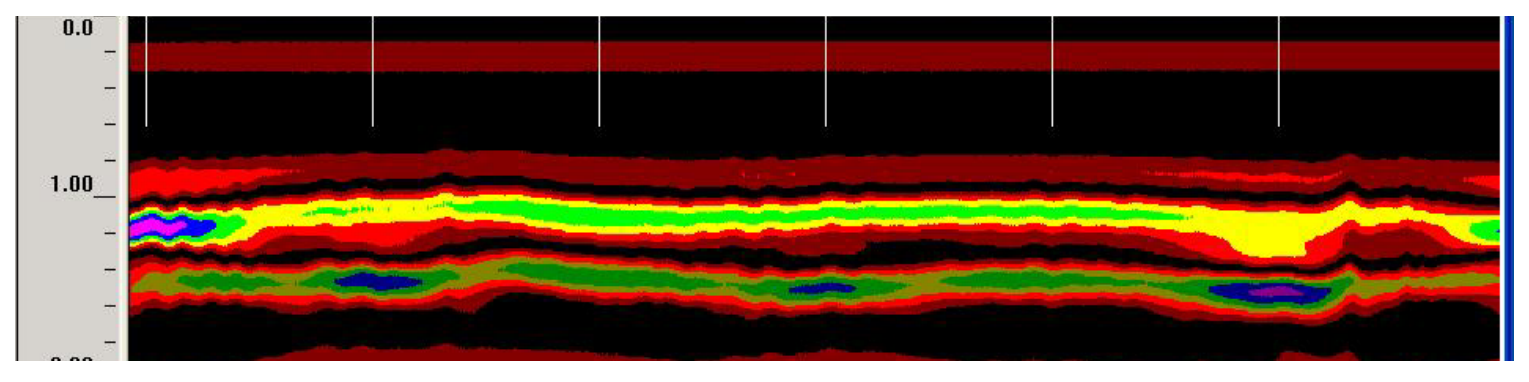

Figure 7.29: 2D image of modules with 8" x 8" x 0.03" and 8" x 8" x 0.09" water-filled debonds.

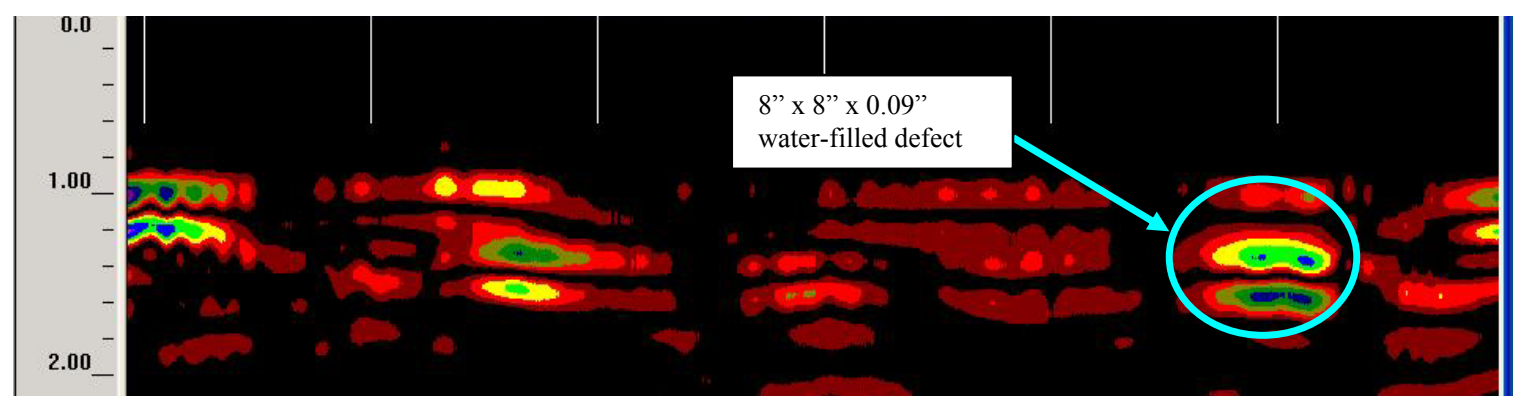

Figure 7.30: 2D image of modules with 8 " 8 " x 0.03 " and 8 " x 8 " x 0.09 " water-filled debonds with FIR. 


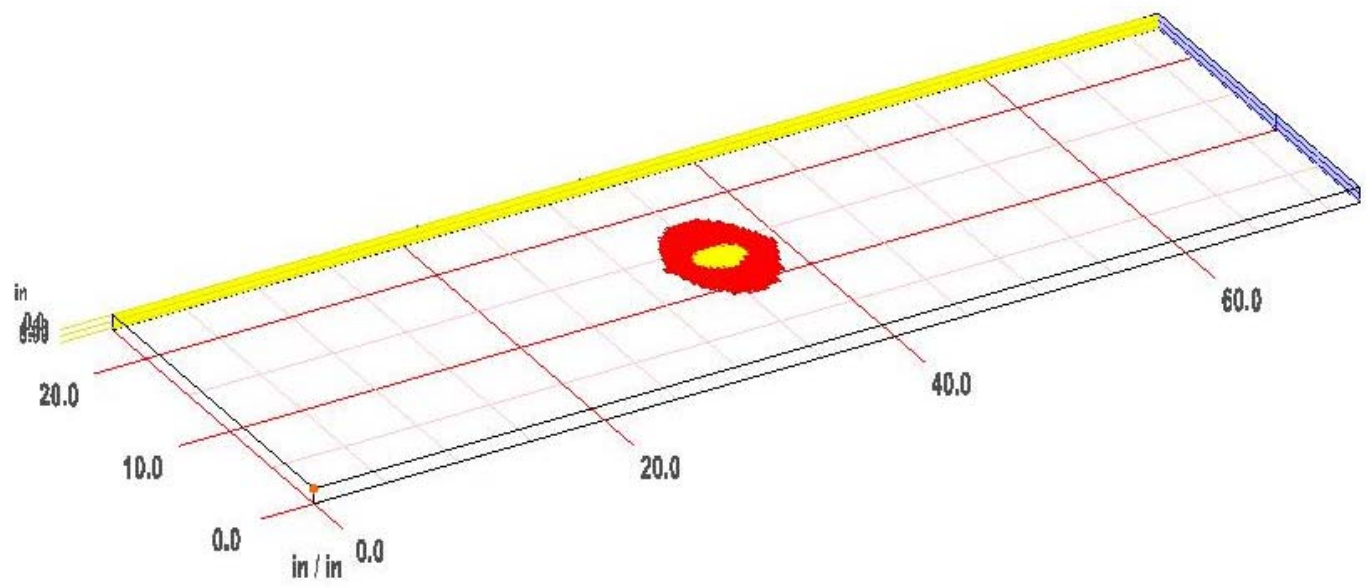

Figure 7.31: 3D plot of the entire deck module with various water-filled defects.

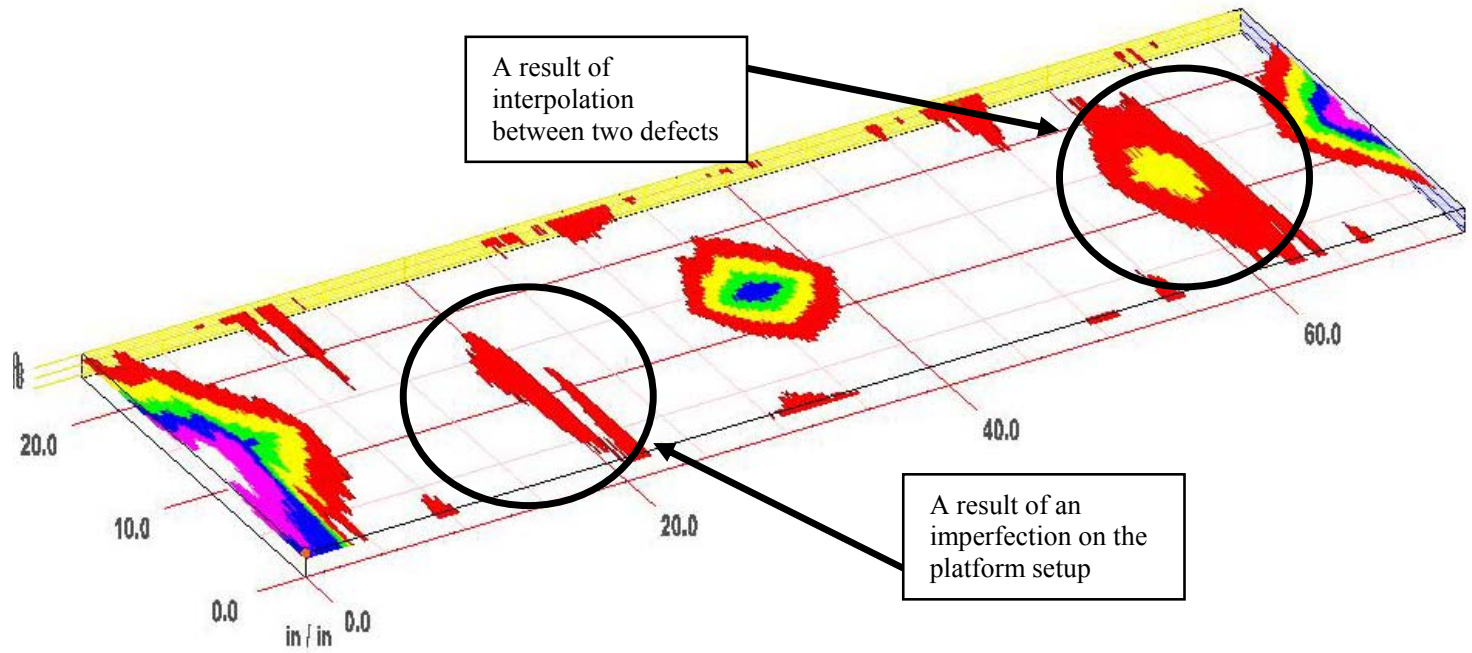

Figure 7.32: FIR 3D plot of the entire deck module with various water-filled defects.

The result from Figure 7.31 is consistent with all the original $2 \mathrm{D}$ images. The only visible water-filled defect that can be detected was the 12 " x 12 " x 0.06 " water-filled debond while the other defects are not as conclusive. While looking at Figure 7.32 and comparing it with the $2 \mathrm{D}$ images, it is clear that FIR did indeed provided a better overall results. Due to the nature of the software (interpolating data between scans), the 8 " $\mathrm{x} 8$ " $\mathrm{x}$ 
0.06 " and 8 " $\times 8$ " x 0.09 " water-filled defects are shown as one big defect on the $3 \mathrm{D}$ plot (Figure 7.32).

Finally, a close look at the signal analysis further strengthens the previous findings. In Figure 7.33, it is clear that 12" x 12" x $0.06 ", 8 "$ x 8" x $0.06 "$, and 8 " x 8" x 0.09" water-filled defects (after subtracting the defect-free waveform) have a significantly higher amplitude compared to other defects with smaller thicknesses. The signal analysis for FIR data sets (Figure 7.34) shows that 12 " x 12 " x 0.06 " water-filled defect can be easily detected but the rest of the waveforms are not as clear cut.

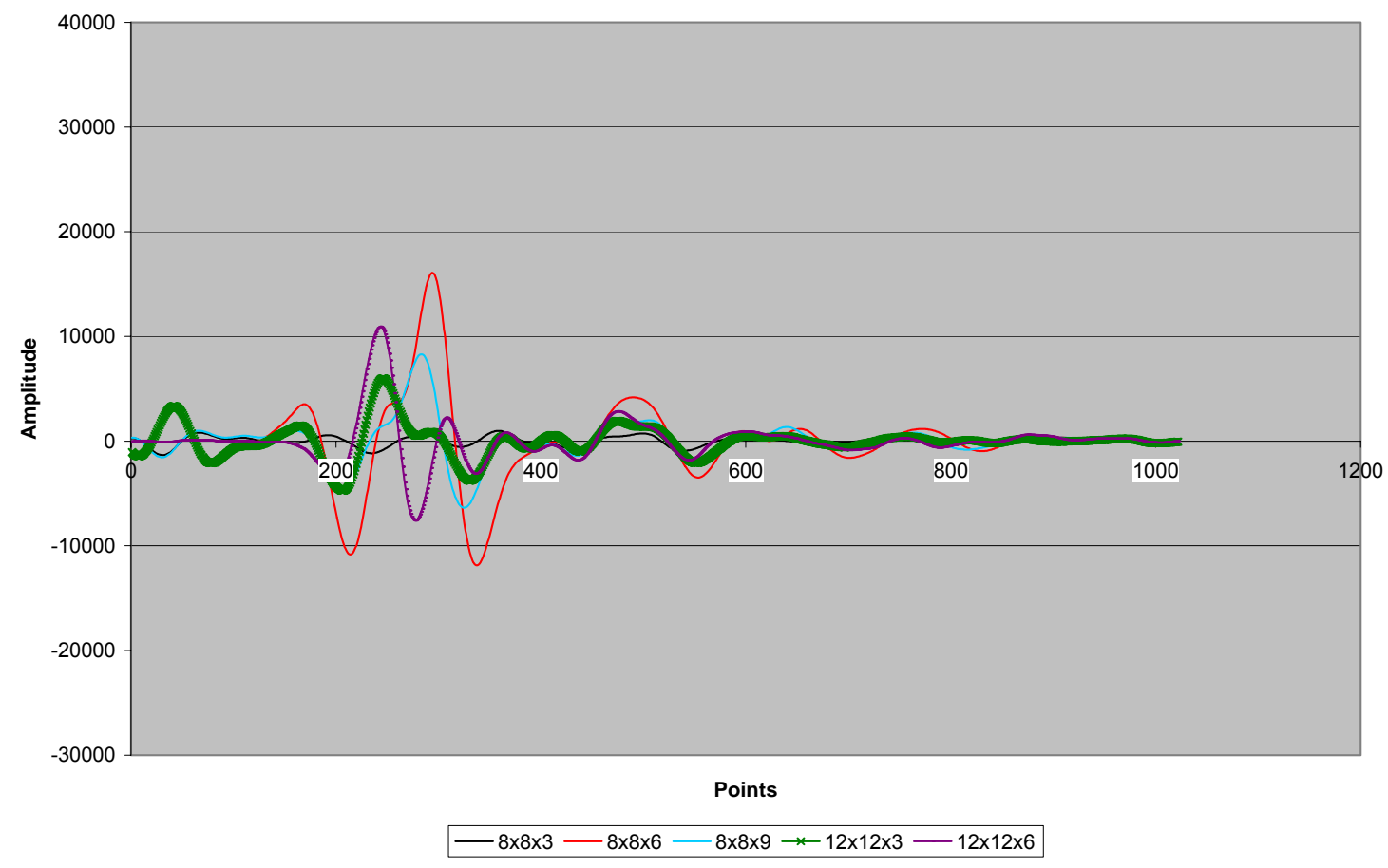

Figure 7.33: Signal analysis of the water-filled defects data. 


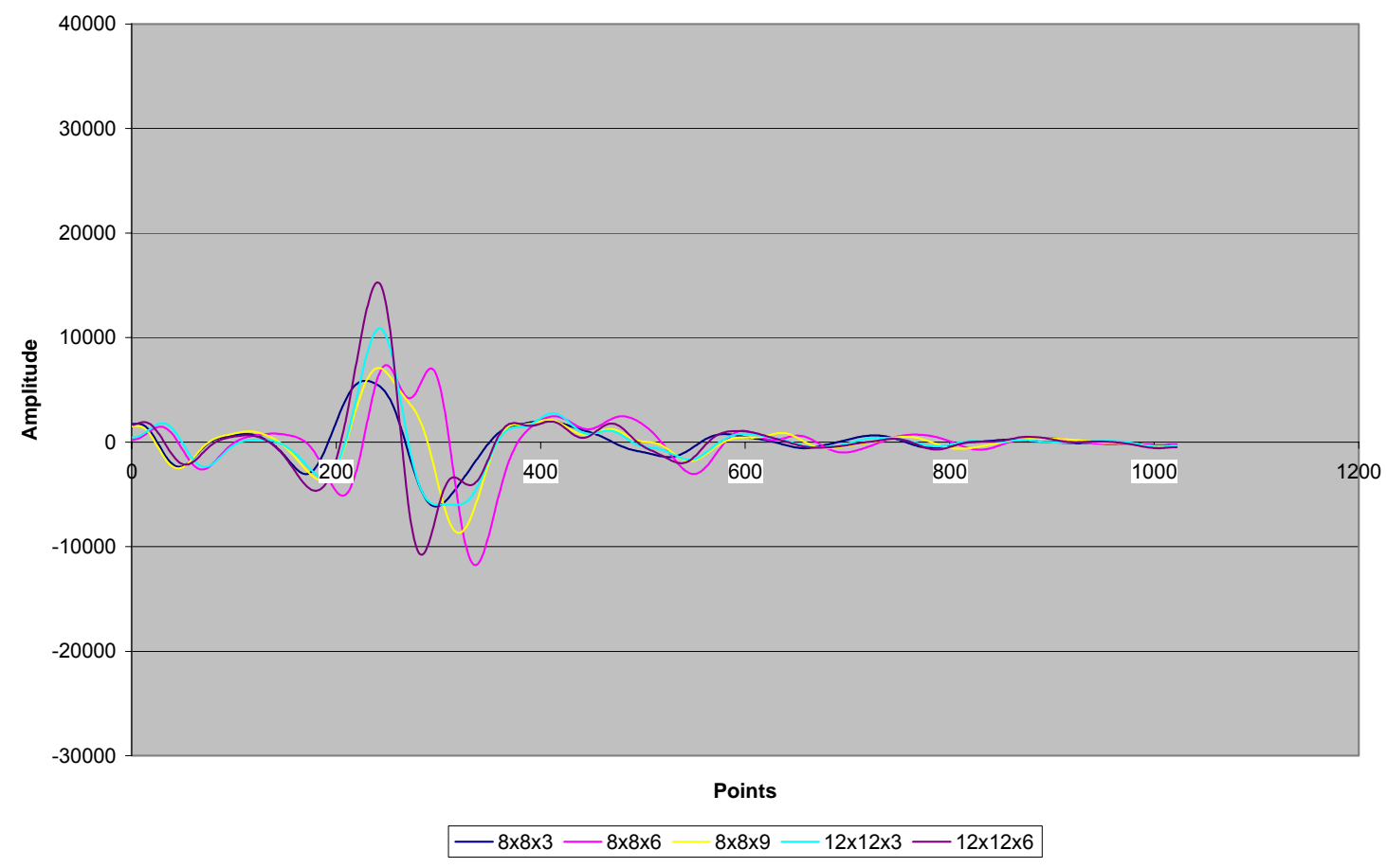

Figure 7.34: Signal analysis of the water-filled defects data with FIR.

In summary, the $2.0 \mathrm{GHz}$ air-coupled antenna GPR system is not capable of detecting any air-filled defects with thicknesses up to 0.09 " $(2.3 \mathrm{~mm})$. By combining the 2D images, 3D images, and signal analysis of both original data and data sets applied with Finite Impulse Response (FIR) filter, the smallest water-filled defect that can be detected by the $2.0 \mathrm{GHz}$ air-coupled antenna is 8 " $\mathrm{x} 8$ " $\mathrm{x} 0.06$ ".

Further GPR tests were all conducted using the $1.5 \mathrm{GHz}$ ground-coupled antenna. All data acquisition was conducted using a 1024 points record length, with a time range of $4 \mathrm{~ns}$ for top flange defect detection and $10 \mathrm{~ns}$ for bottom flange defect detection. A further discussions on the parameter settings is provided later in section 7.2

The following defect layout (Figure 7.35) was used to conduct a series of GPR tests using the $1.5 \mathrm{GHz}$ ground-coupled antenna. 


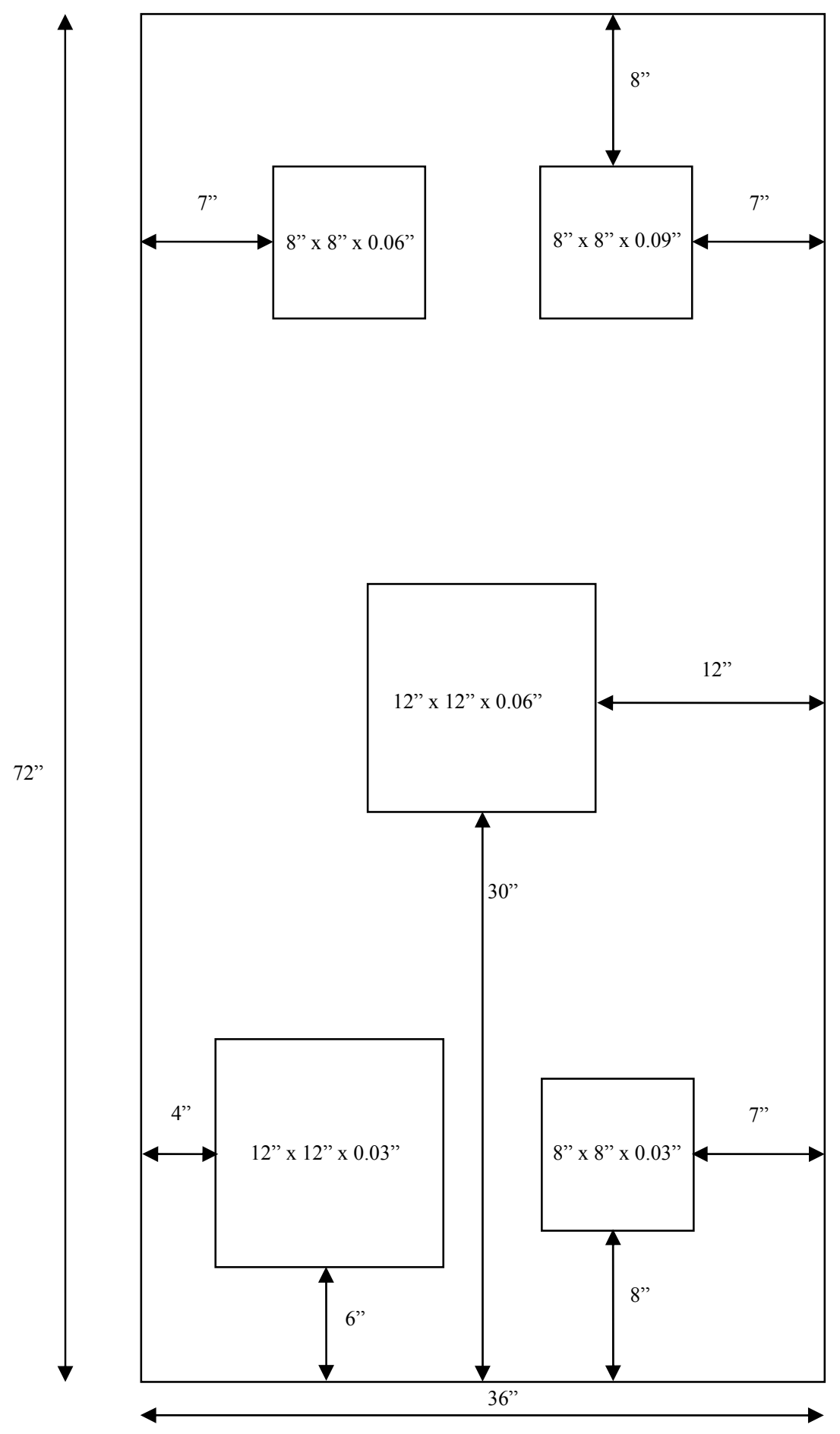

Figure 7.35: Layout of the defect for $1.5 \mathrm{GHz}$ ground-coupled antenna GPR test. 
As seen in Figures 7.36 and 7.37, the two water-filled debonds can be clearly identified. The same process was repeated to identify other water-filled debonds and the results are shown in Figures 7.38 to 7.41 . It can be seen from these figures that the GPR scans obtained after applying the FIR filter to the original data sets show the defect locations much more clearly. Notice that the size of the defect shown on the GPR scans is directly dependent on the thickness of the defect rather than its planar size.

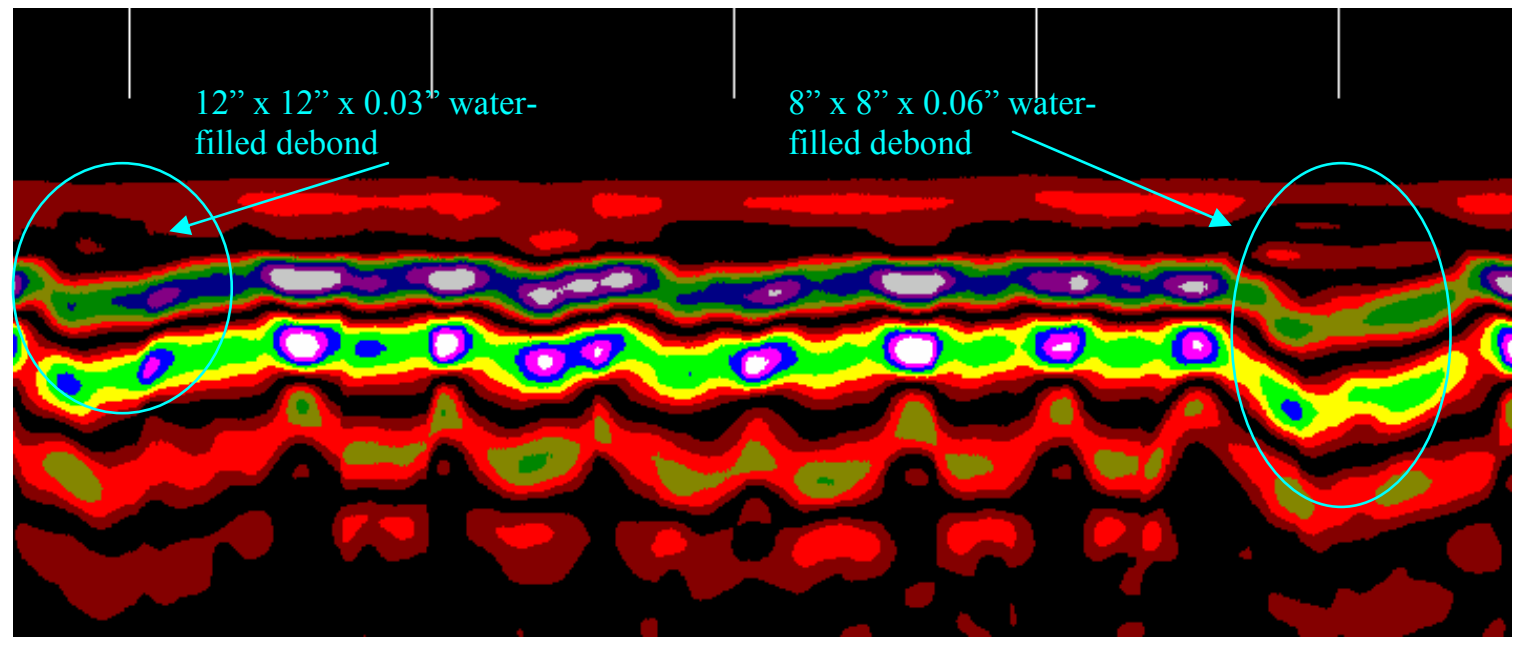

Figure 7.36: Results of GPR test showing 12 " 12 " x 0.03 " and 8 ” 8 " x 0.06 " water-filled debonds.

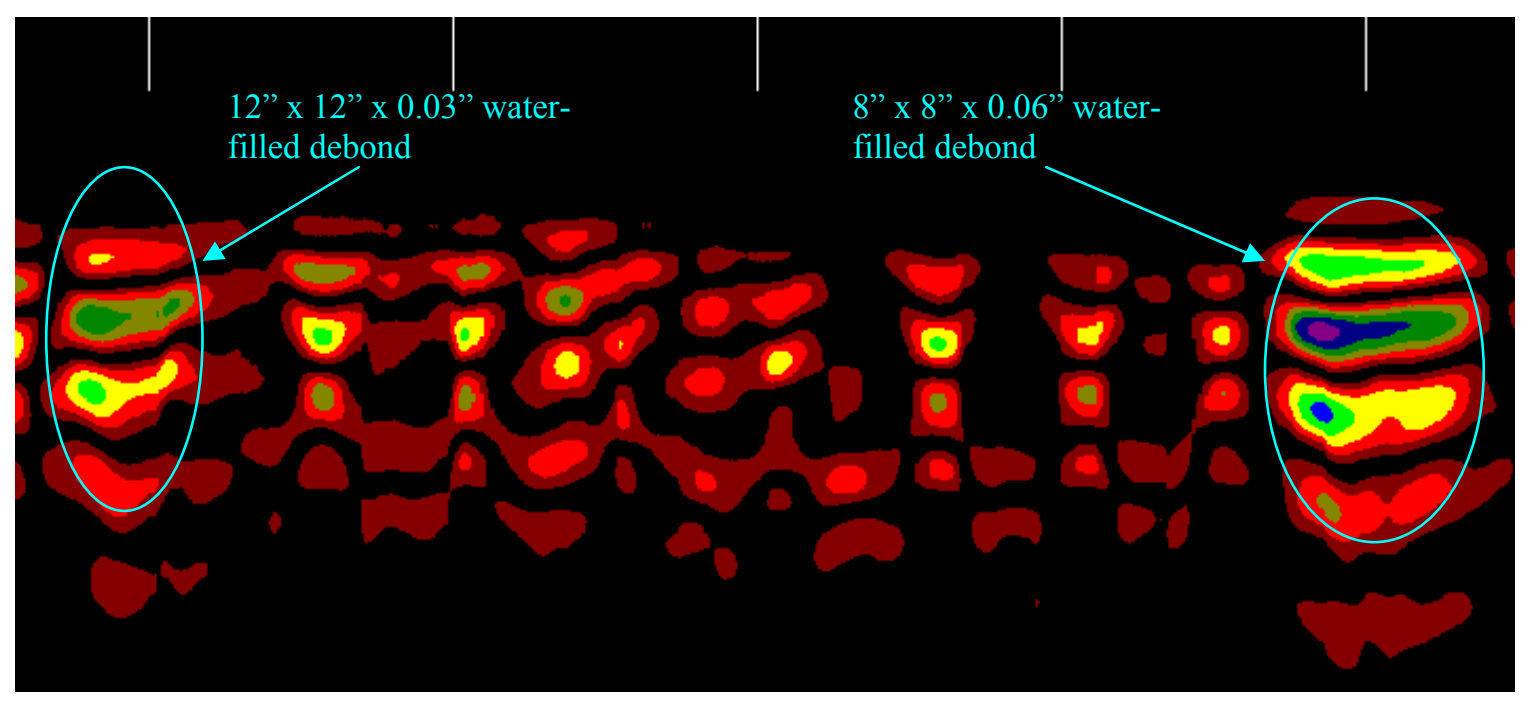

Figure 7.37: Results of GPR test (FIR) showing 12" x 12 " x 0.03 " and 8 " x 8 " x 0.06 " water-filled debond. 


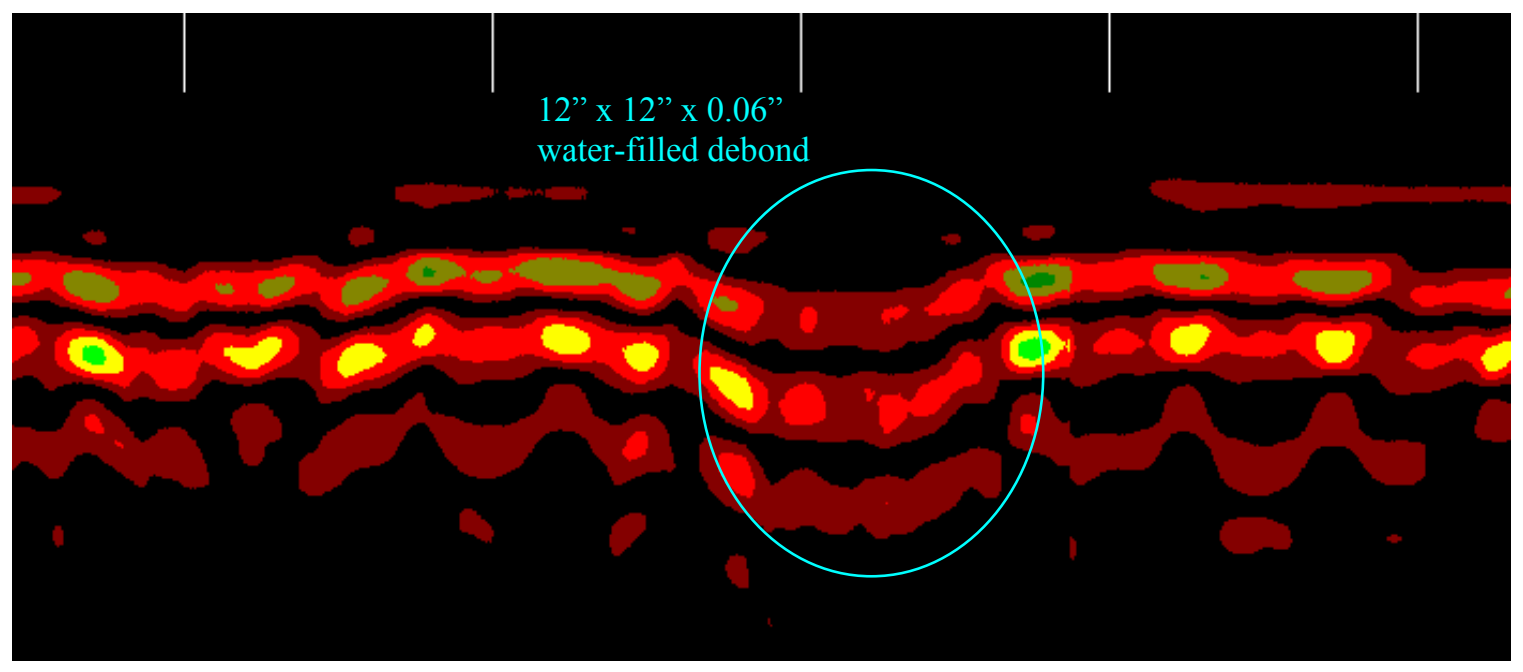

Figure 7.38: Results of GPR test showing 12" x 12" x 0.06 " water-filled debond.

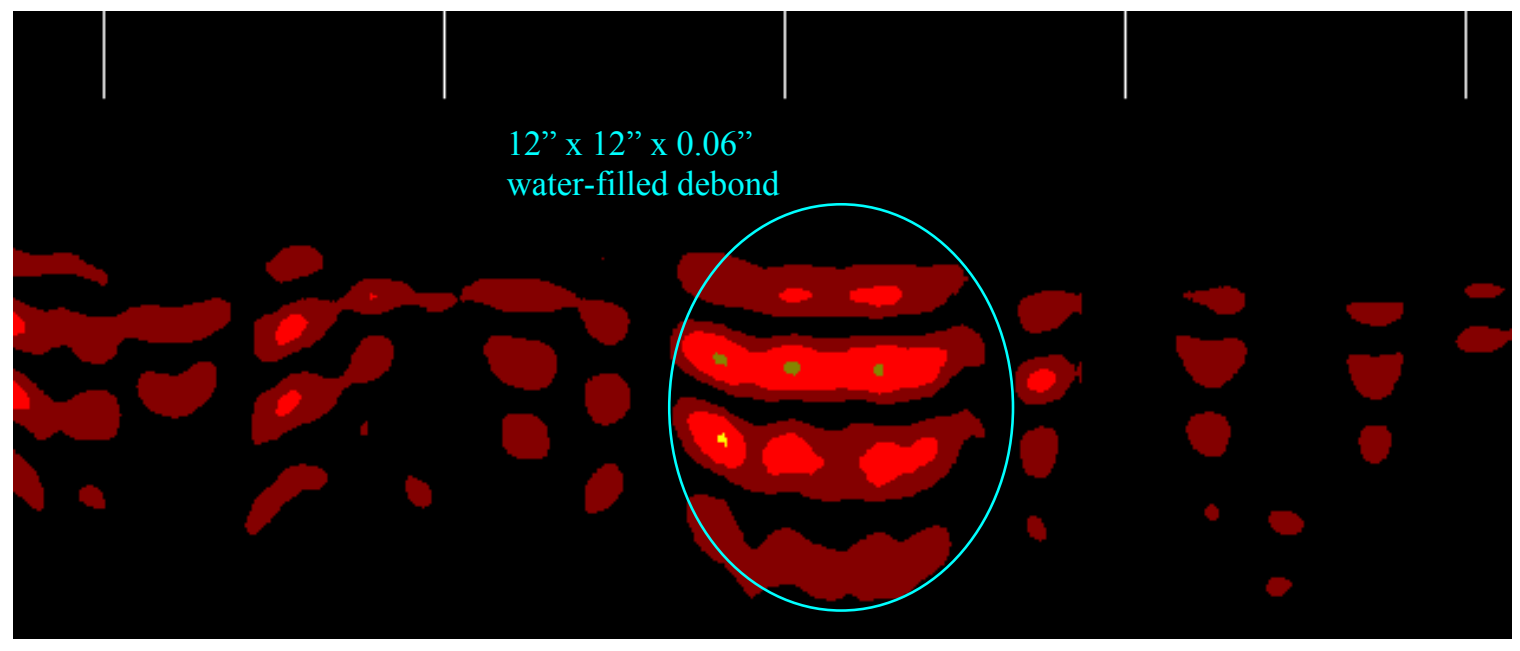

Figure 7.39: Results of GPR test (FIR) showing 12" x 12" x 0.06 " water-filled debond. 


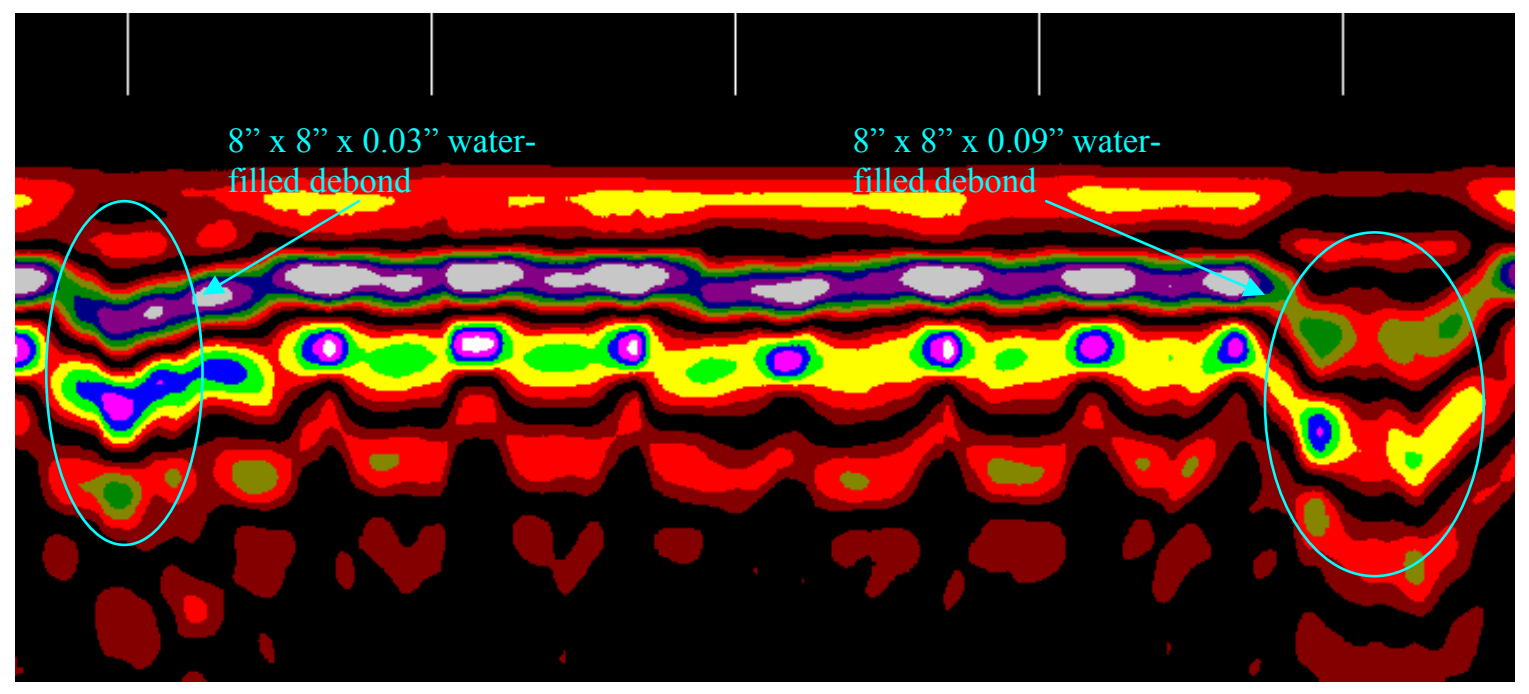

Figure 7.40: Results of GPR test showing 8 " x 8" x 0.03 " and 8 " x 8" x 0.09 ” water-filled debond.

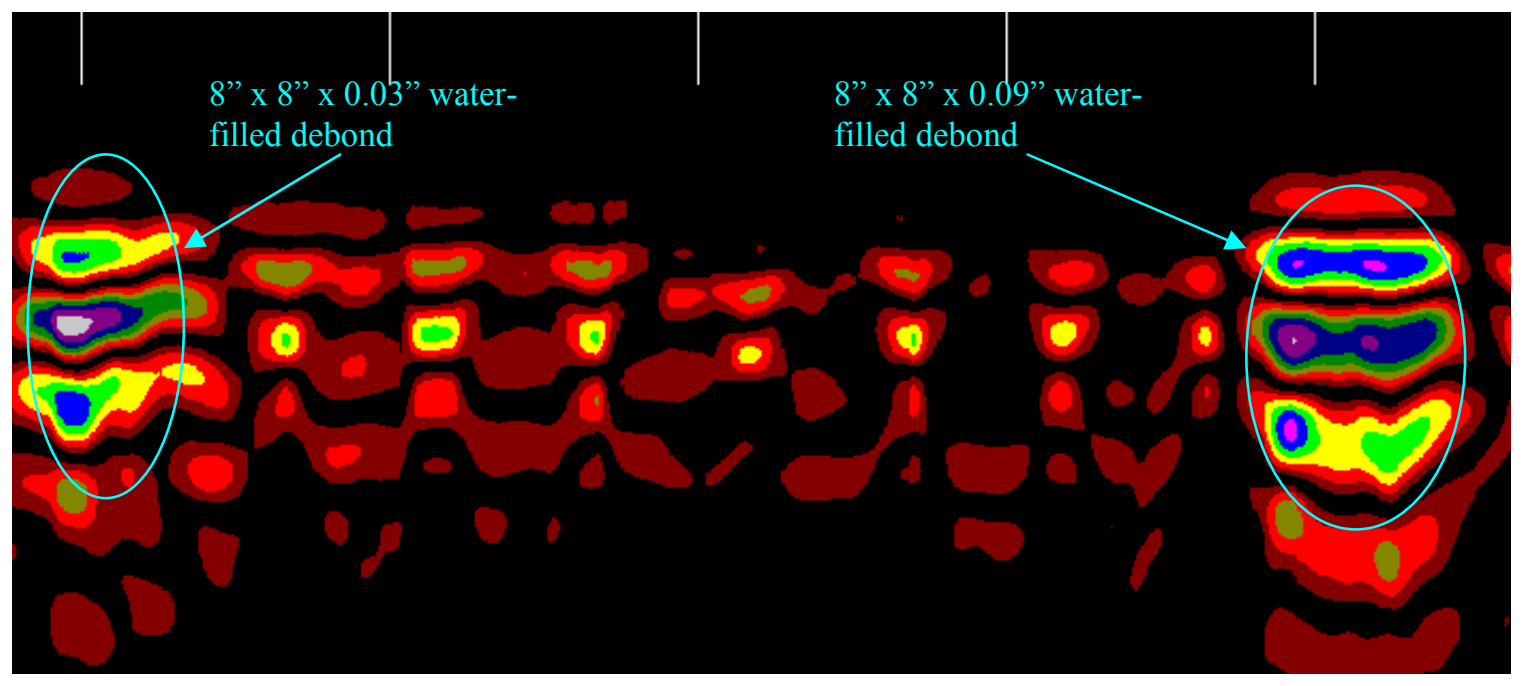

Figure 7.41: Results of GPR test (FIR) showing 8 " x 8" x 0.03 ” and 8” x 8" x 0.09 ” water-filled debond. 


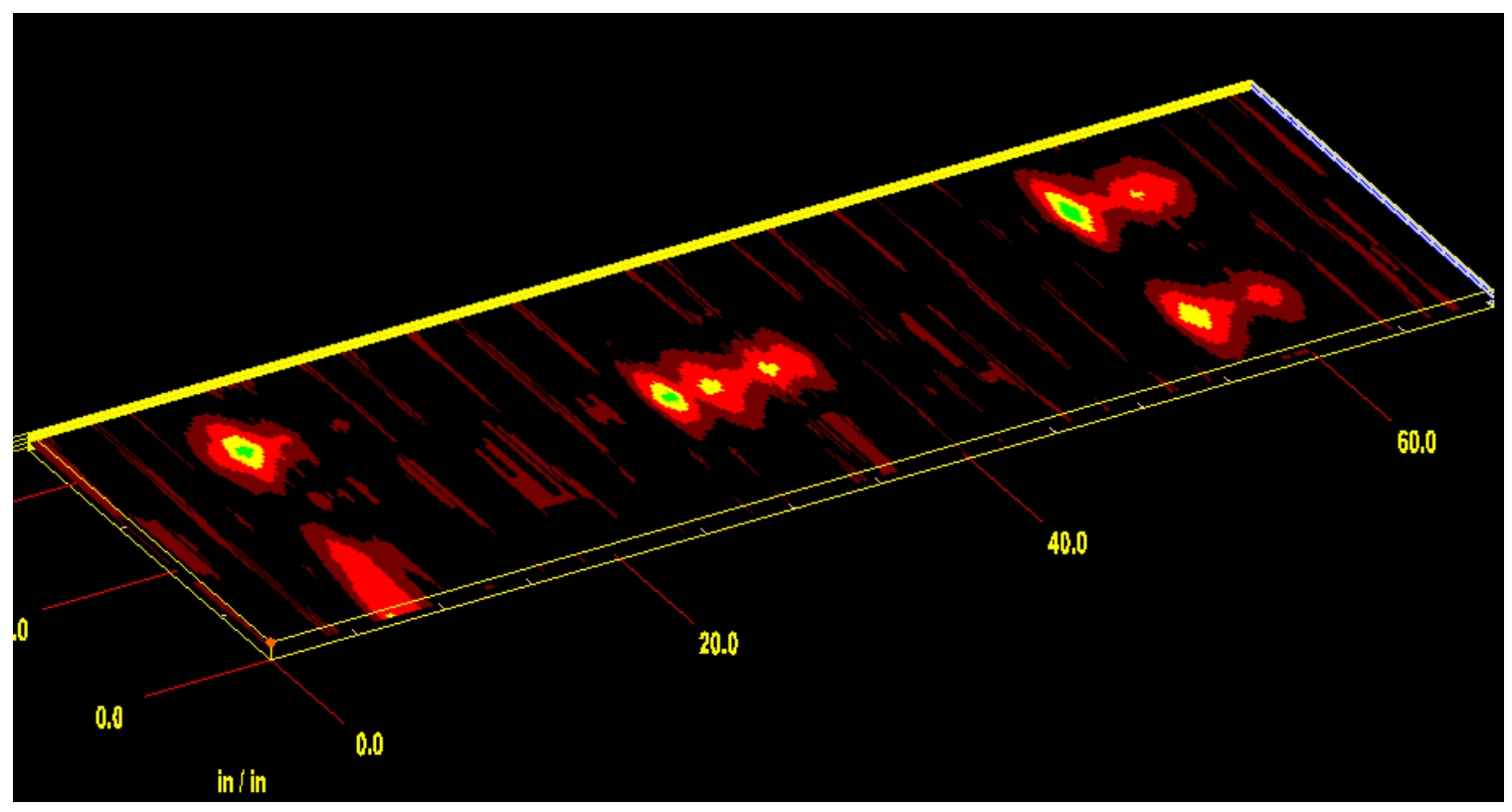

Figure 7.42: 3D map showing all the water-filled debonds.

Figure 7.42 shows the 3D map of the location of all the water-filled debonds. As seen above, the 3D representation is an extremely convenient tool to identify defect locations.

The $1.5 \mathrm{GHz}$ antenna proved to have no problems in identifying the larger defects (8" x 8 " and $12 "$ x 12"). A further study of the $1.5 \mathrm{GHz}$ ground-coupled antenna was done by performing a GPR test on an FRP deck with the debond configuration shown in Figure 7.43. This configuration included many smaller size defects.

As shown in Figures 7.44 and 7.45, the smaller water-filled debonds can also be detected. Notice in Figure 7.44, only three distinctive distorted regions can be identified from the original scan while there are four water-filled debonds altogether. Whereas in Figure 7.45, all four water-filled debonds can be clearly identified. This indicates that while the original 2D scan may be able to display the small water-filled defects, it is best first apply the FIR filter to eliminate any system noise thus greatly improving the results.

Also note that no air-filled debonds could be clearly identified in these scans. 


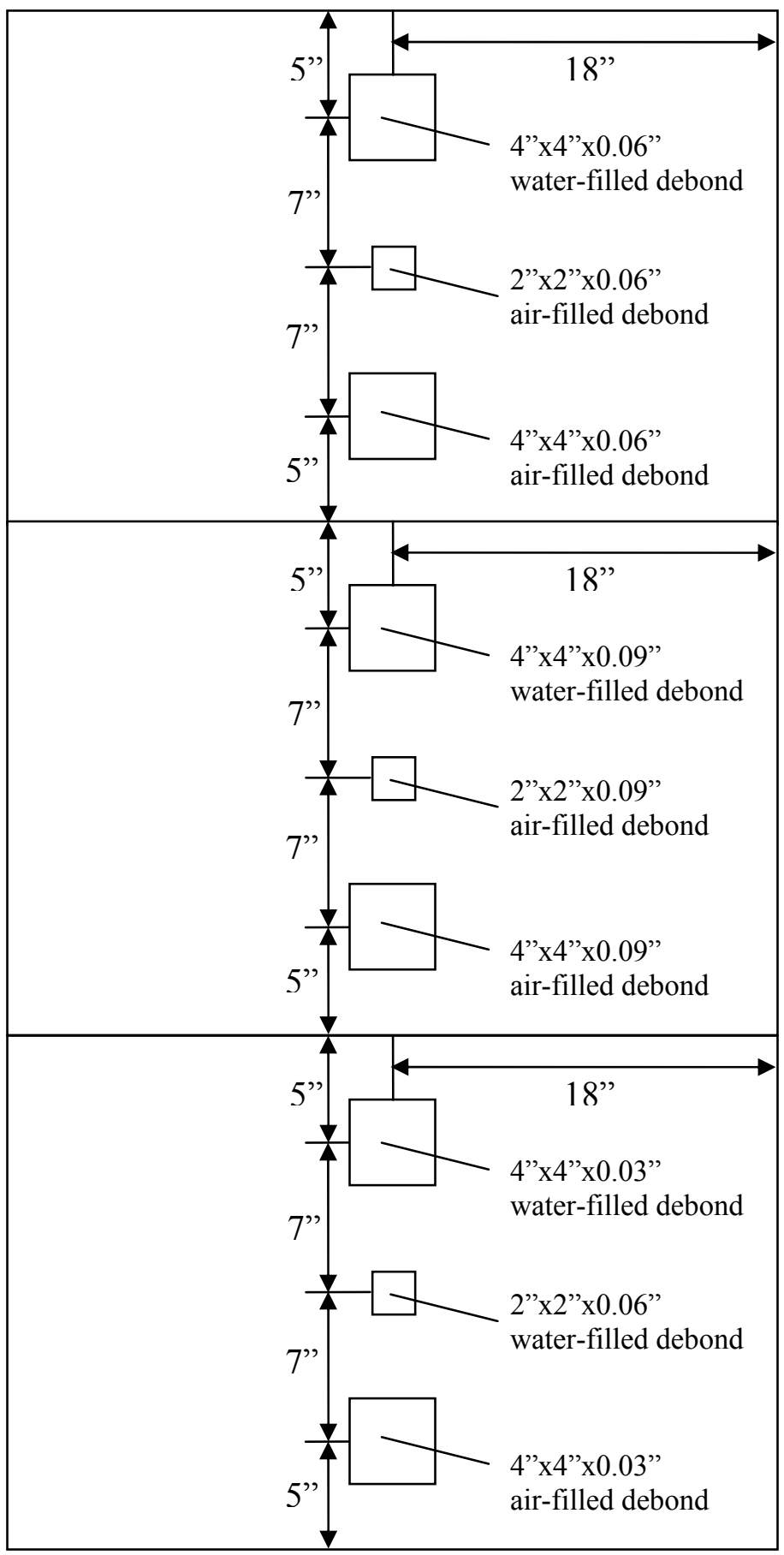

Figure 7.43: Layout of the defects for $1.5 \mathrm{GHz}$ ground-coupled antenna GPR test. 


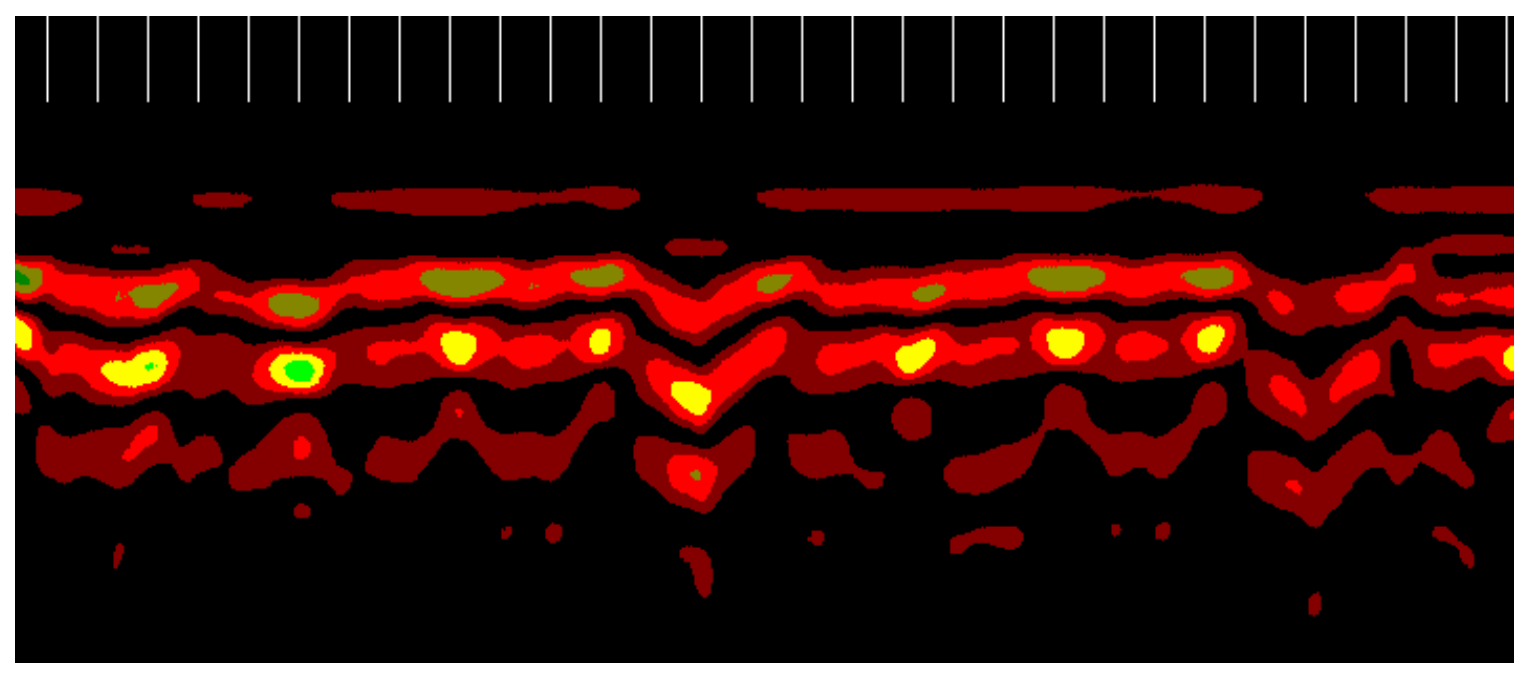

Figure 7.44: Results of GPR test showing smaller sized water-filled debonds.

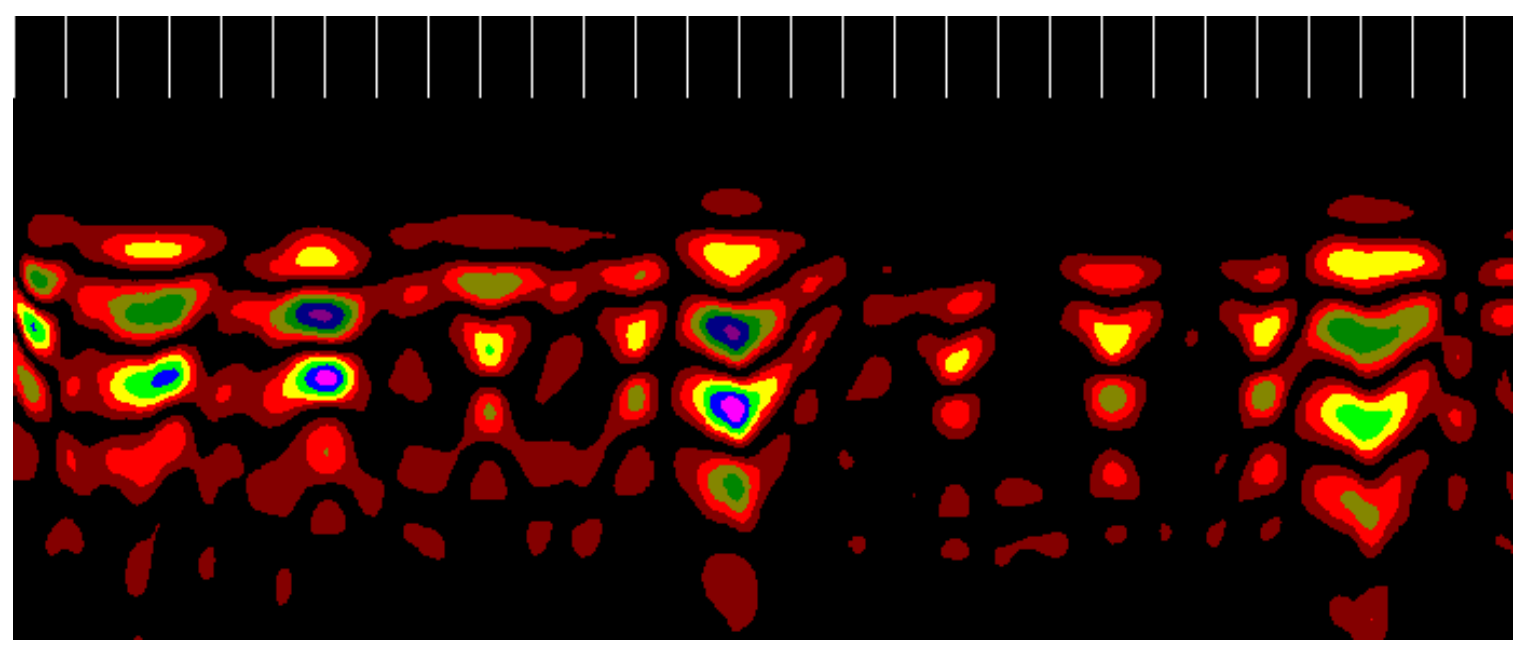

Figure 7.45: Results of GPR test (FIR) showing smaller sized water-filled debonds. 


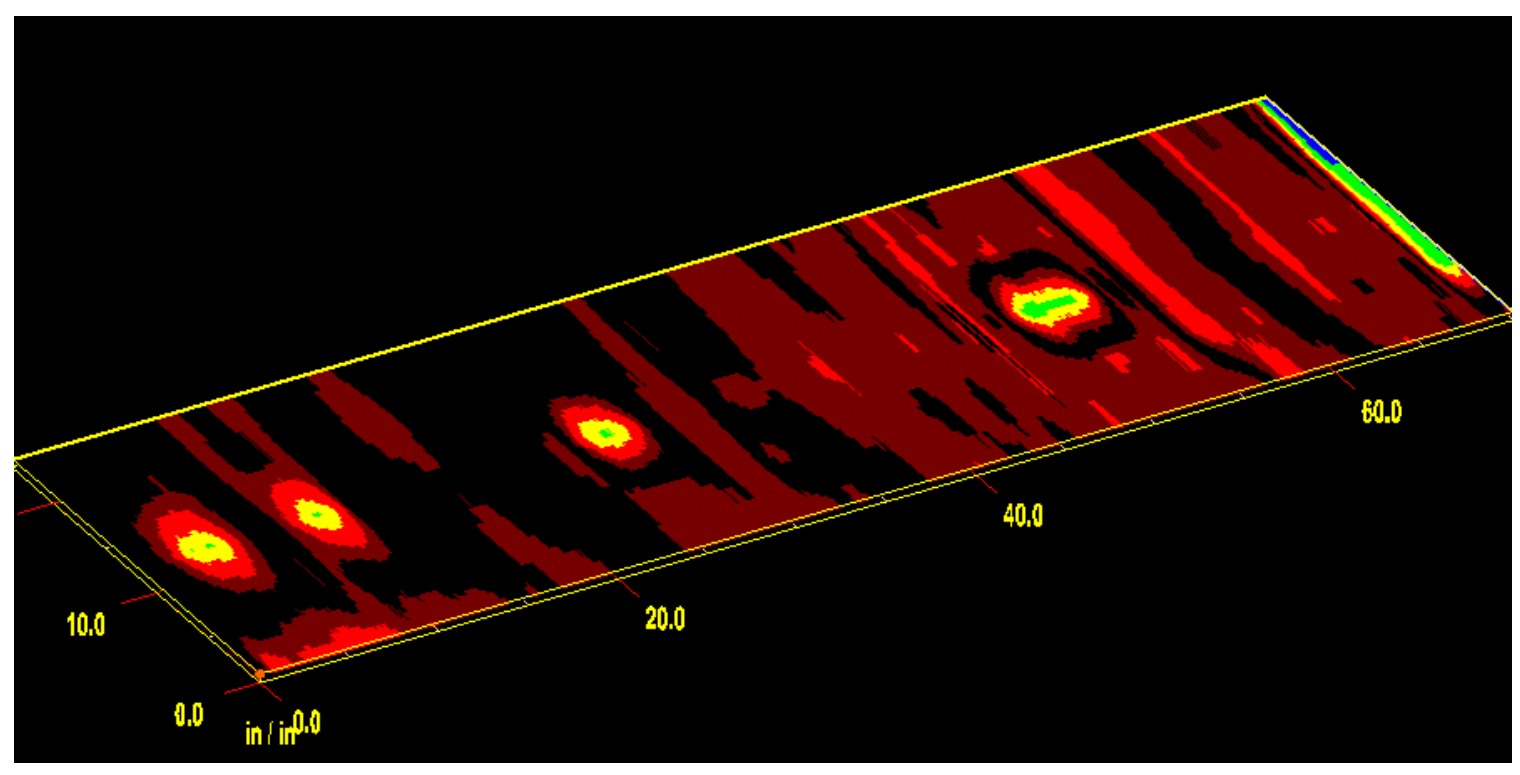

Figure 7.46: 3D map showing all the water-filled debonds.

As with the larger water-filled debonds, a 3D map can be constructed through the data sets applied with FIR. As shown in Figure 7.46, all the four water-filled debonds are clearly displayed on the 3D map at their respective locations.

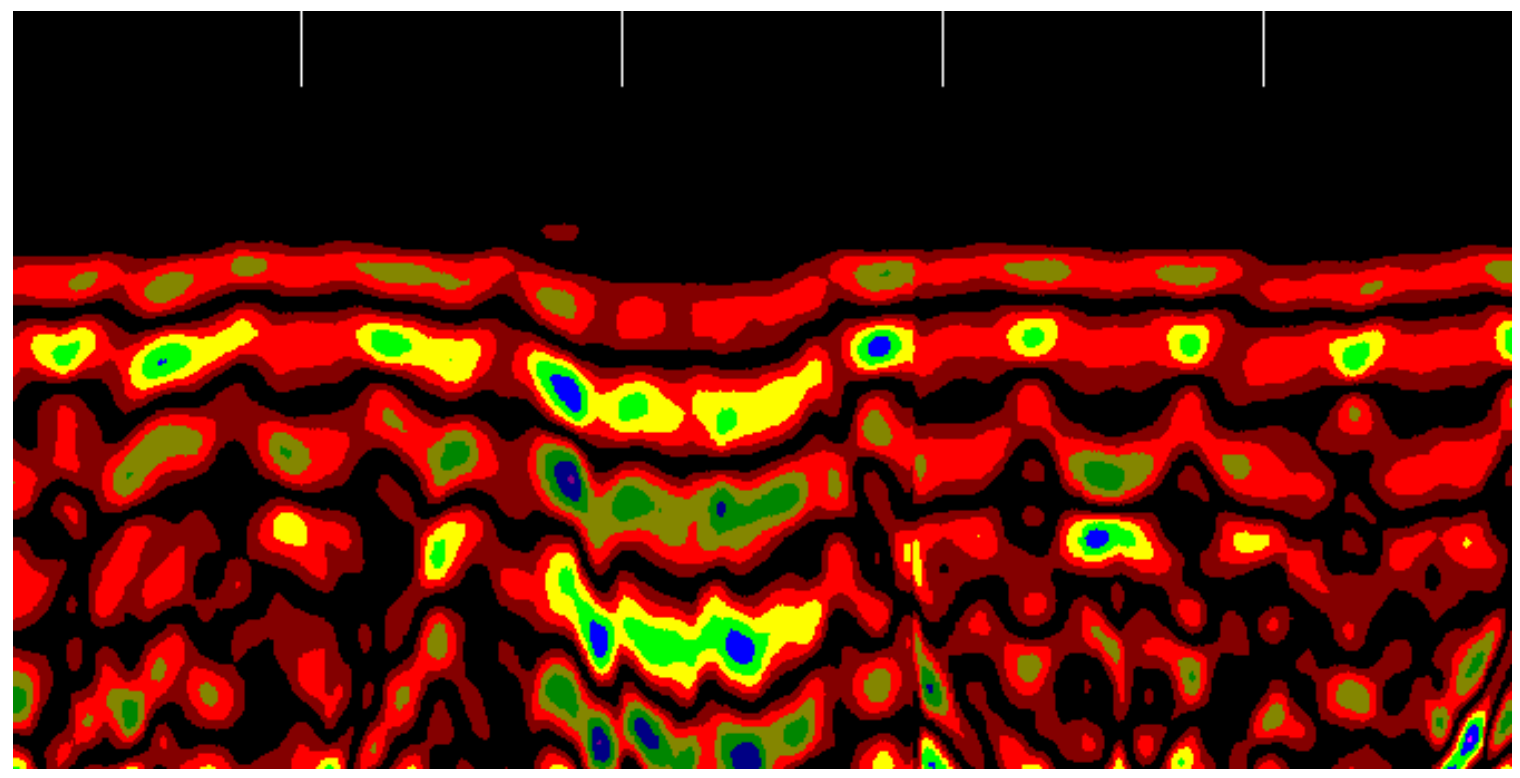

Figure 7.47(a): 2D GPR data collected using linear gain setting. 


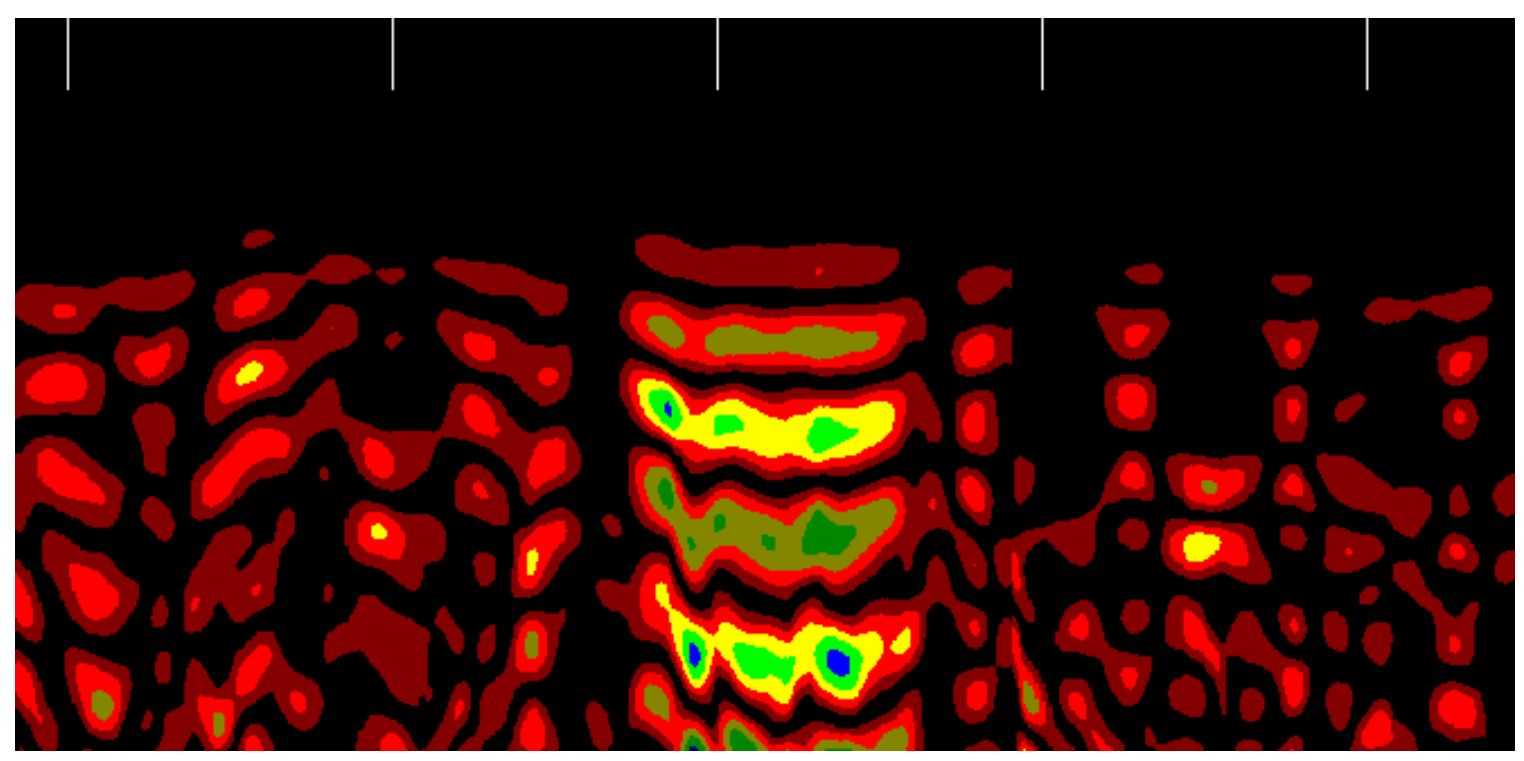

Figure 7.47(b): 2D GPR data (FIR) collected using linear gain setting.

Figures $7.47(a)$ and $7.47($ b) show the 2D data acquired using a "linear" gain (increasing with depth), while all previous data was acquired using constant gain. By comparison with Figures 7.38 and 7.39 (constant gain), the linear gain data in Figures 7.47(a) and 7.47(b) certainly provided a larger intensity. However, the original or raw linear gain data (before applying FIR filter) in Figure 7.47(a) is more difficult to interpret than the raw constant gain data in Figure 7.38.

\subsubsection{Delamination Detection Using GPR}

One of the goals of this research was to determine the ability to detect delamination within the top and bottom flange of FRP decks without requiring access to the bottom side of the deck, that is, by conducting nondestructive measurements only from the top surface of the FRP deck. Infrared thermography could not be used in this case since there is a wide gap between the top and bottom flanges which affect the heat transfer through the deck, and also since the infrared technique can only detect detects 
over shallow depths. GPR, on the other hand, provides deeper penetration capability. Therefore, its use was investigated for defect detection in the bottom flange of an FRP deck.

A 4" (101.6mm) thick low profile FRP deck (with properties as described earlier) was structurally tested until failure occurred (Figures 7.48(a) and 7.48(b)). The defectfree wearing surface modules were placed on the top of the deck. The $1.5 \mathrm{GHz}$ groundcoupled antenna was used to scan the failed deck. Ten nanosecond range was used instead of the previous five nanosecond range since the bottom flange was of concern.

The 3D profile (Figure 7.49) reveals the exact location of the failed region within the low profile FRP deck. Though the failed region is clearly observable due to the severity of the damage, it is encouraging to note that GPR could detect defects as far down as 4" (101.6mm). To verify the result, FIR filtering was applied to the 2D data and this $2 \mathrm{D}$ scan was observed. The 2D profile of the GPR data revealed the same information. It can be seen from Figure 7.50 (failed region) and Figure 7.51 (defect-free region) that the severity of the damage causes the radar echo from the webs to be attenuated extensively. The web echo signals from the FRP deck can be seen clearly in the defect-free 2D profile (Figure 7.51) but the web echo signals attenuated significantly at damaged regions (Figure 7.50 ). While the 2D profile may not show the bottom flange clearly, the 3D profile of this experiment proves that GPR has potential for locating defects at greater depths (web and possibly bottom flange). 


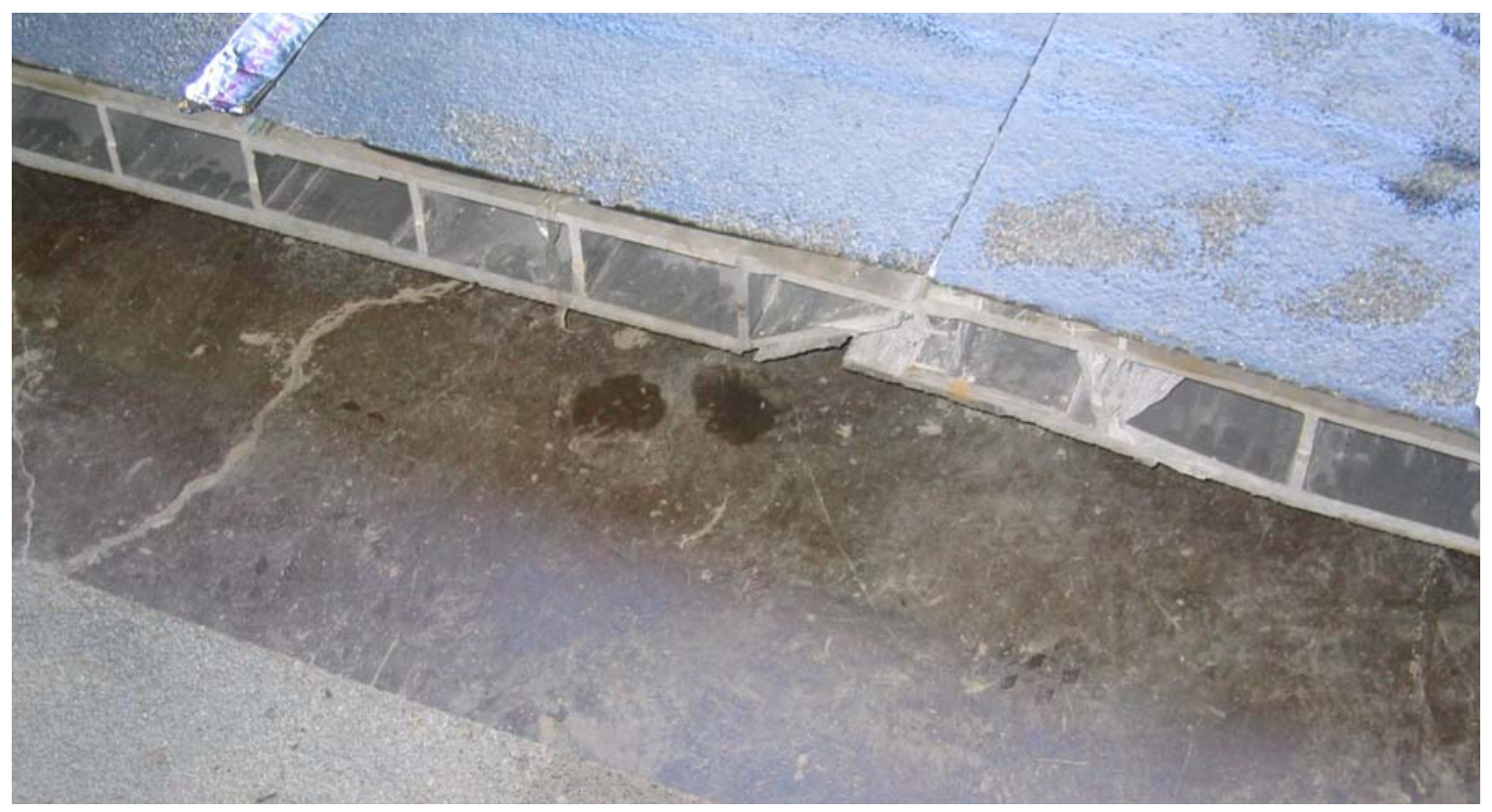

(a)

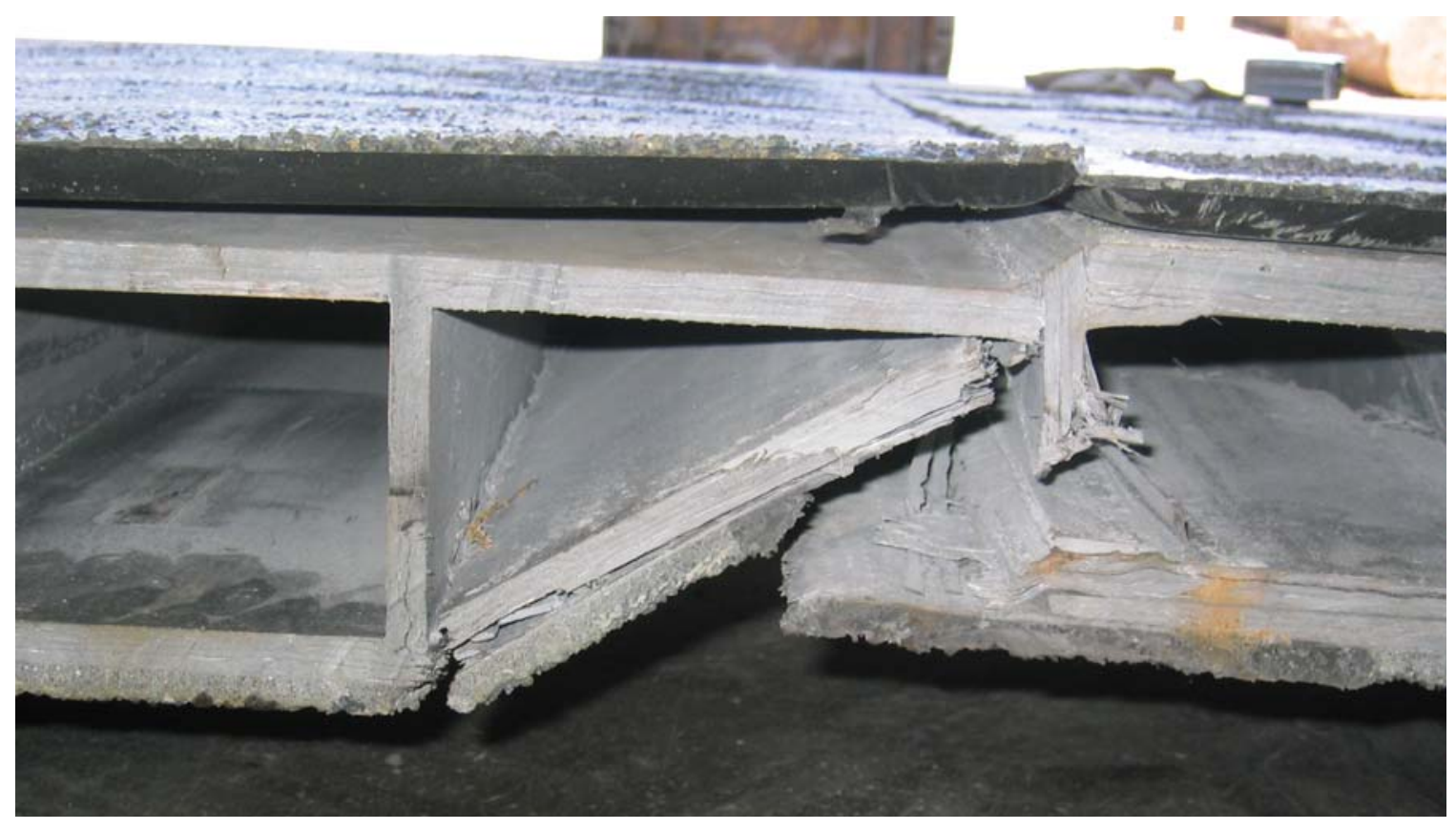

(b)

Figure 7.48: Pictures showing 4" thick low profile deck that was loaded until failure. 


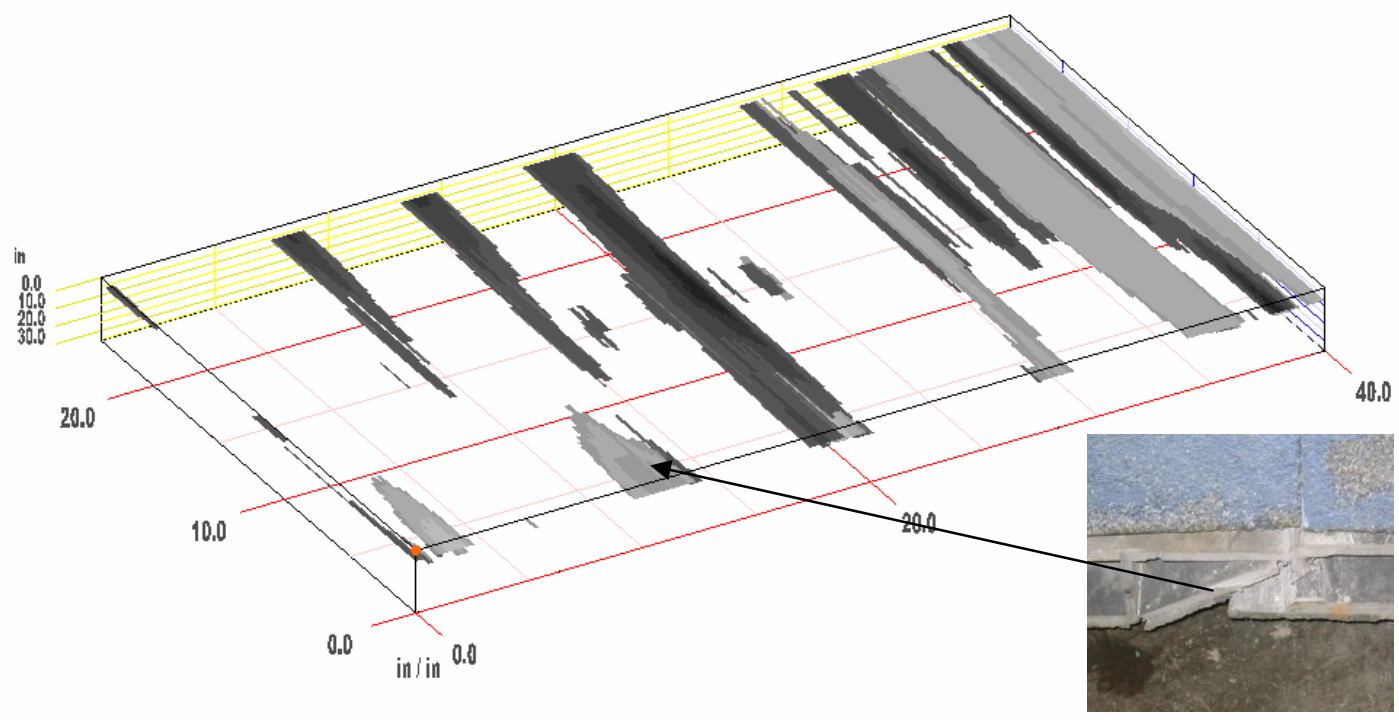

Figure 7.49: 3D GPR profile of the 4" thick low profile failed deck.

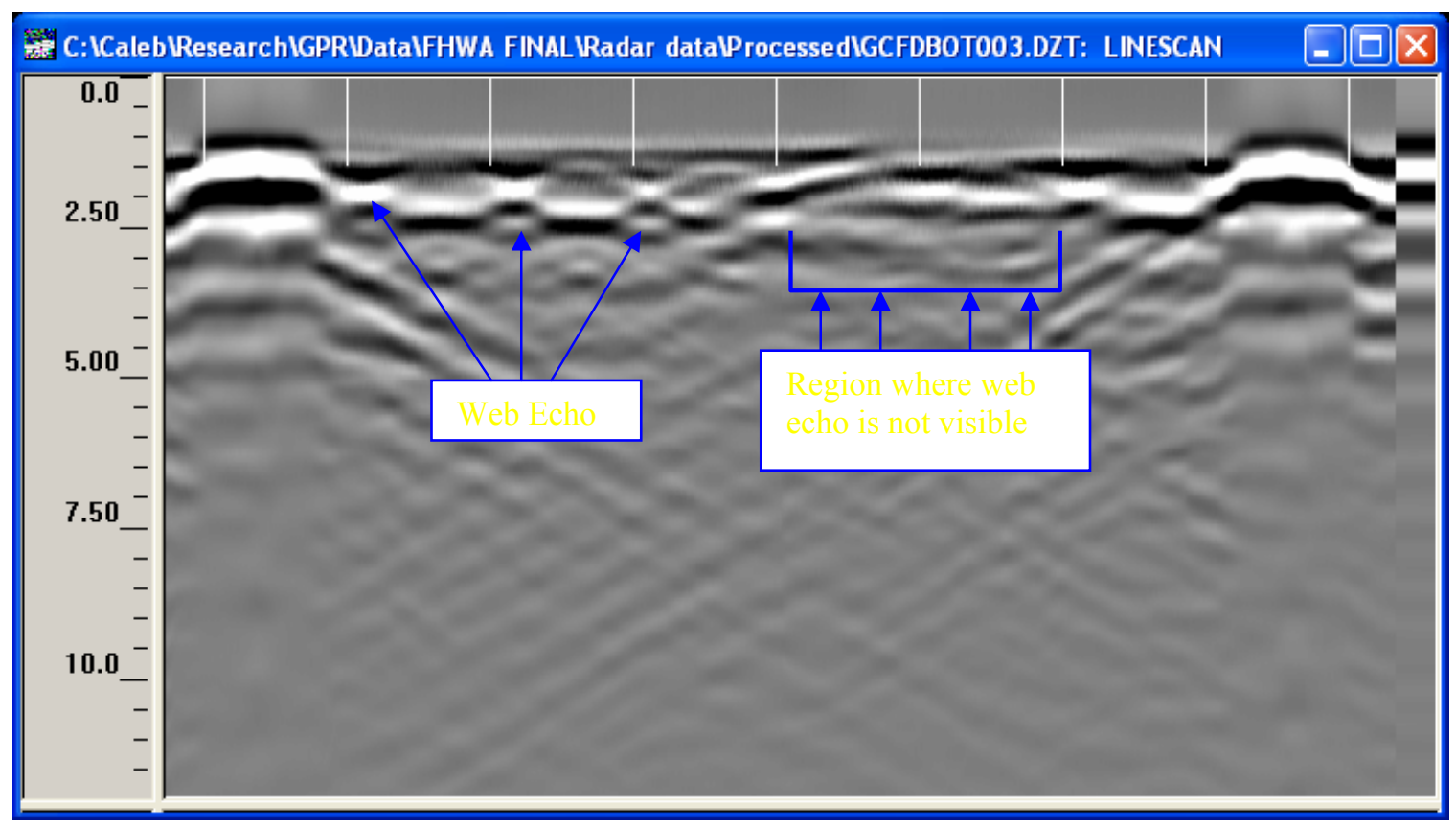

Figure 7.50: 2D GPR profile of the "failed region" in the 4" thick low profile failed deck. 


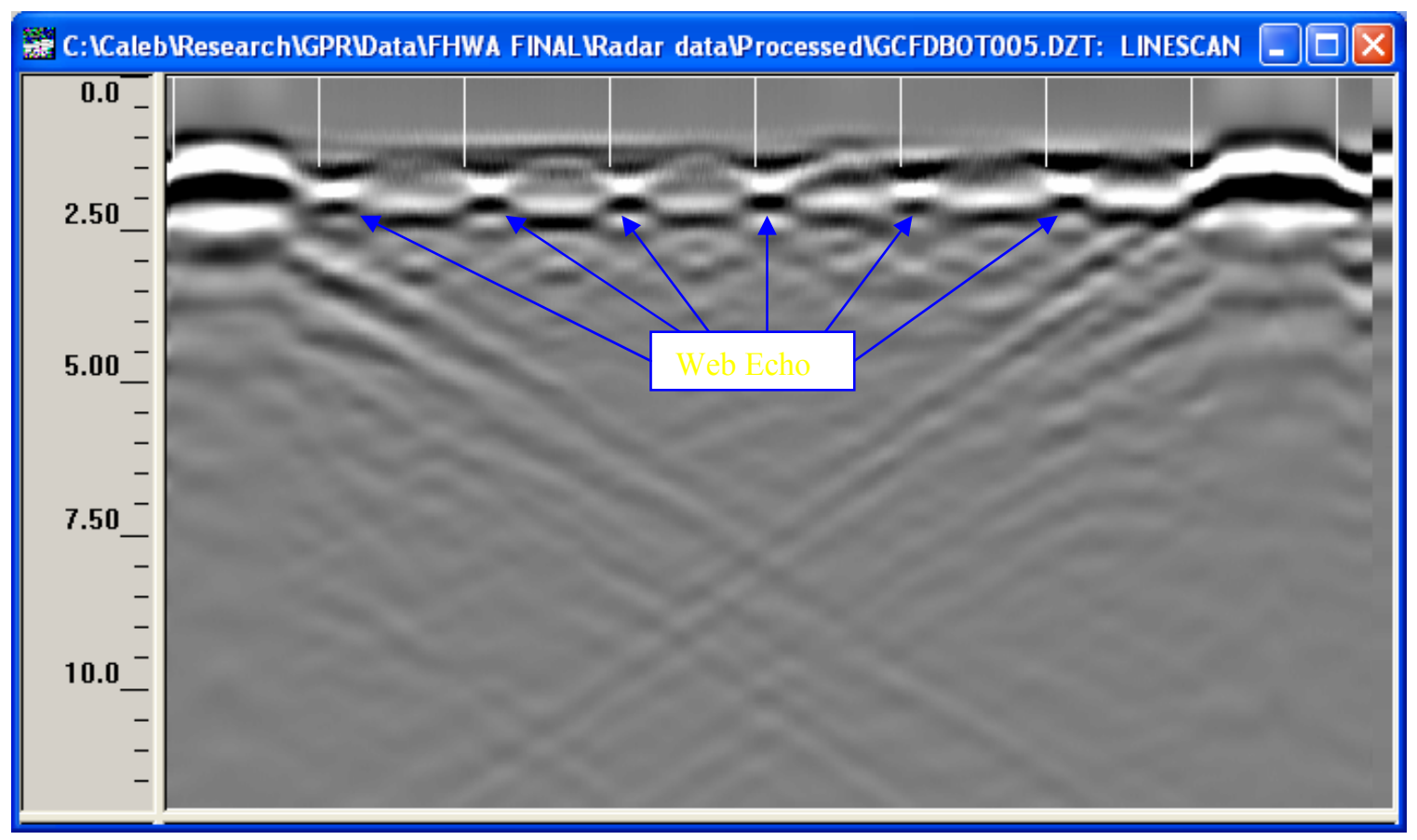

Figure 7.51: 2D GPR profile of the "defect-free region" in the 4" thick low profile failed deck.

To further investigate the viability of GPR as a tool to detect delaminations within top and bottom flanges of a FRP bridge deck, a delaminated module was used to perform GPR test using $1.5 \mathrm{GHz}$ antenna. Figure 7.52 shows the configuration of the delaminations (also see Figures 4.13 to 4.16 in Chapter 4). A defect-free wearing surface was placed over the top flange (with embedded delaminations) of the FRP deck. Figure 7.53 shows the GPR scan in which only the 3 " $\mathrm{x} 3$ " $\mathrm{x} 0.06$ " water-filled delamination is clearly visible. Apparently, it is easy to locate a 3" x 3 " x 0.06 " water-filled delamination within the top flange. However, GPR is unable to detect the air-filled delamination.

The bottom flange delamination could be easily simulated by inverting the entire bridge deck module upside down and supported on both ends to ensure a gap between the bottom face of the bridge deck module and the ground. In this case, the wearing surface was still placed over the top flange but the delaminations were inside the bottom flange. Another round of GPR test was conducted using a linear gain (to magnify the weakened 
signal towards the bottom). Comparing Figures 7.54 and 7.55 , it is clear that the $1.5 \mathrm{GHz}$ ground-coupled antenna is capable of detecting water-filled delaminations within the bottom flange of FRP decks. Notice, however, that unlike the case of delamination within the top flange (Figure 7.53), the location of the water-filled delamination cannot be pinpointed exactly in case of the bottom flange (Figure 7.55) since the radar echo is more

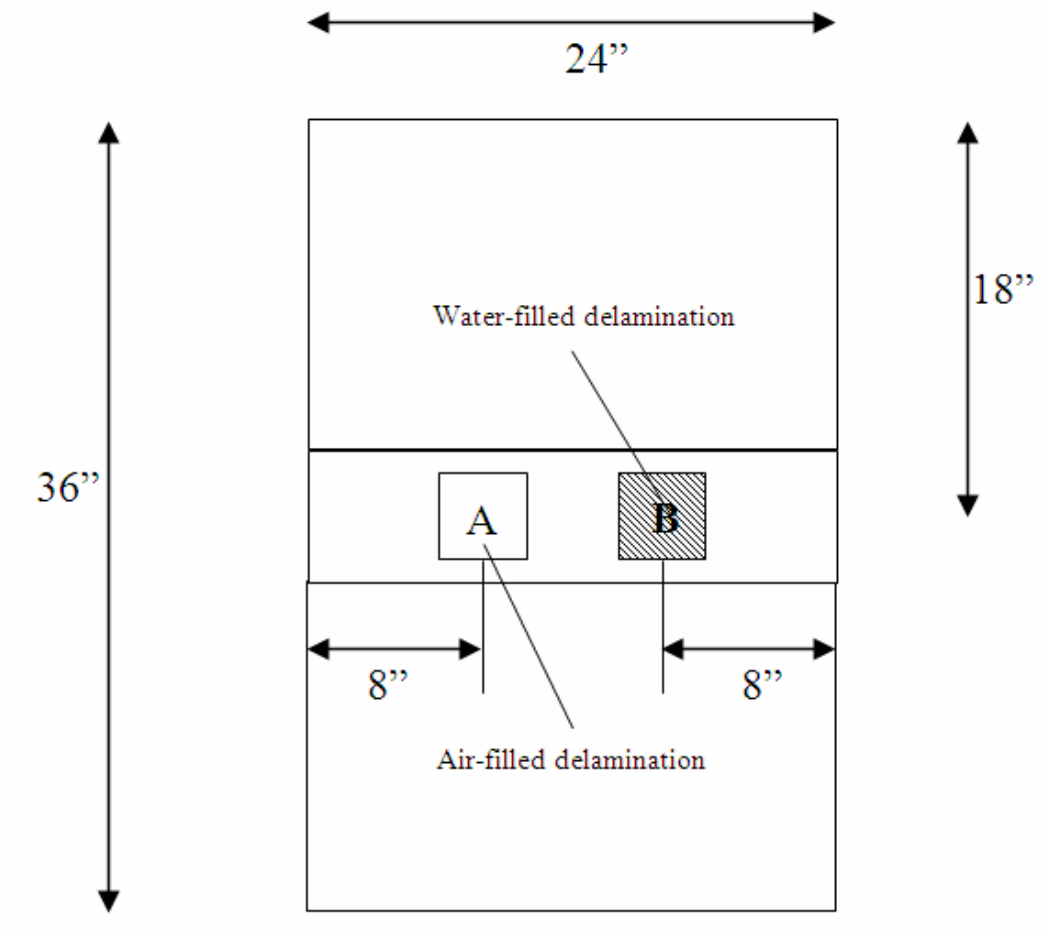

Figure 7.52: Defect layout of delamination module.

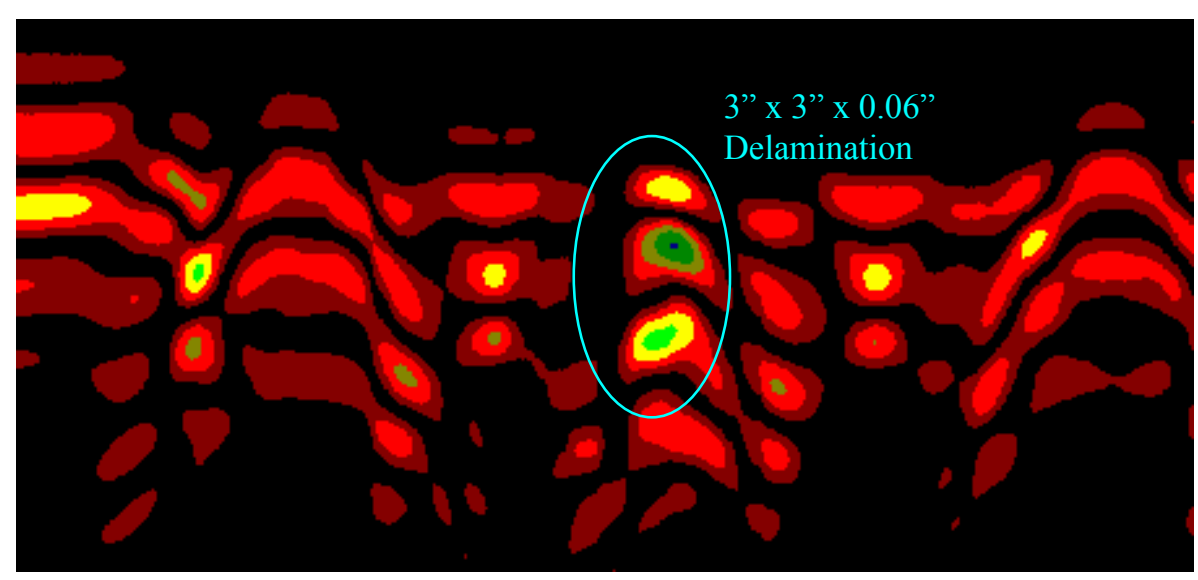

Figure 7.53: 2D scan of delamination embedded within top flange of FRP bridge deck module. 


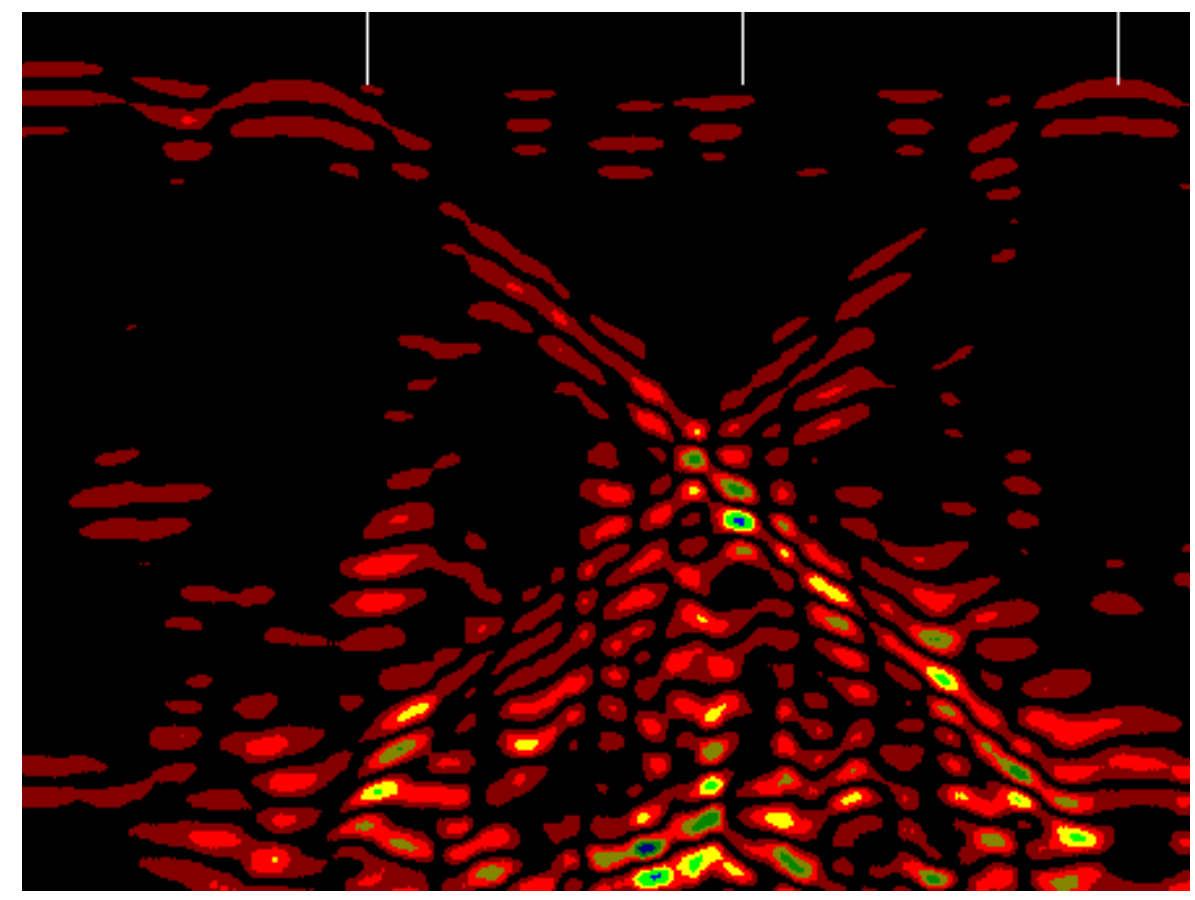

Figure 7.54: 2D scan of defect free FRP bridge deck module.

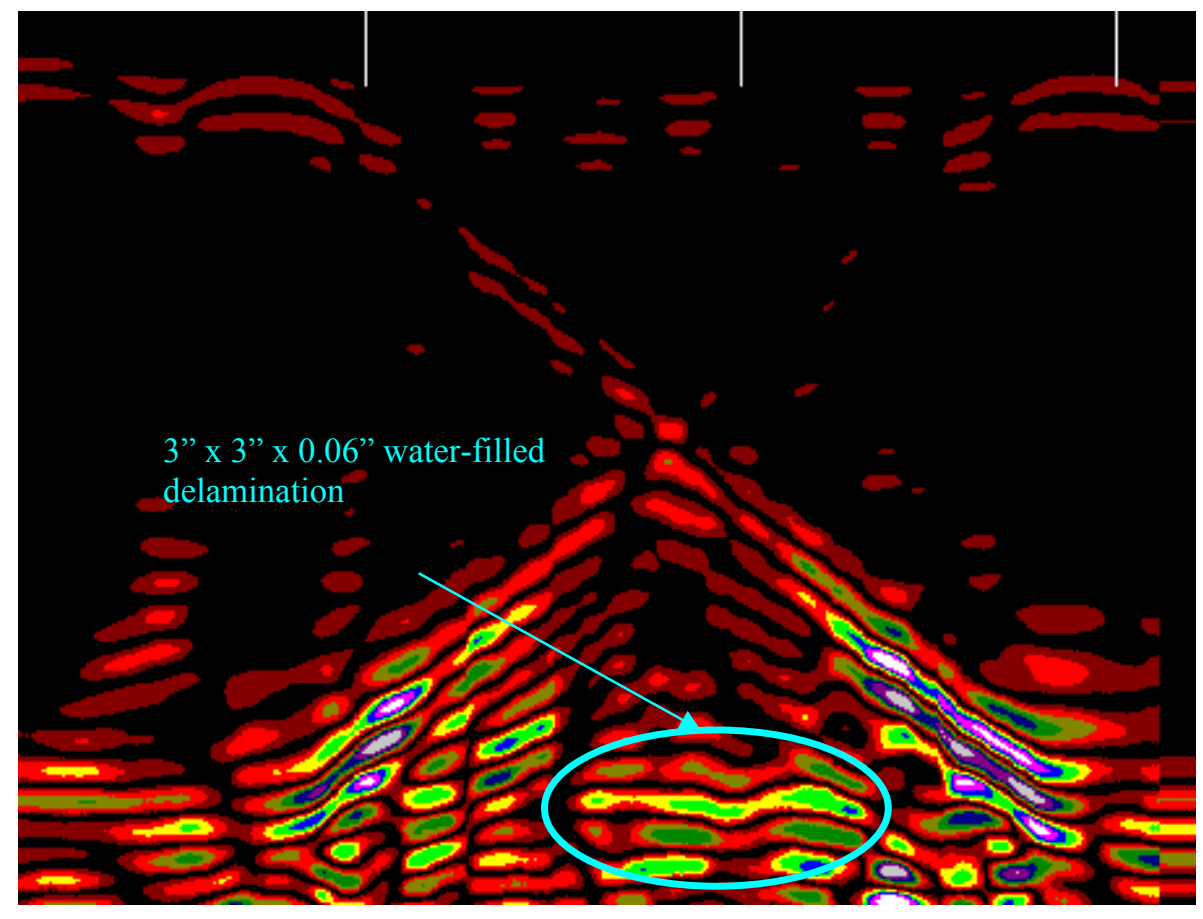

Figure 7.55: 2D scan of delamination embedded within bottom flange of FRP bridge deck module. 
spread out due to larger depth of the delamination. Furthermore, it was noted that it is actually harder to locate water-filled delamination in the top flange with a linear gain data set (Figure 7.56) than a constant gain data (Figure 7.53). On the other hand, linear gain helped in detecting delamination in the bottom flange (Figure 7.55). The 3D map using constant gain is shown in Figure 7.57 and the water-filled delamination is clearly visible.

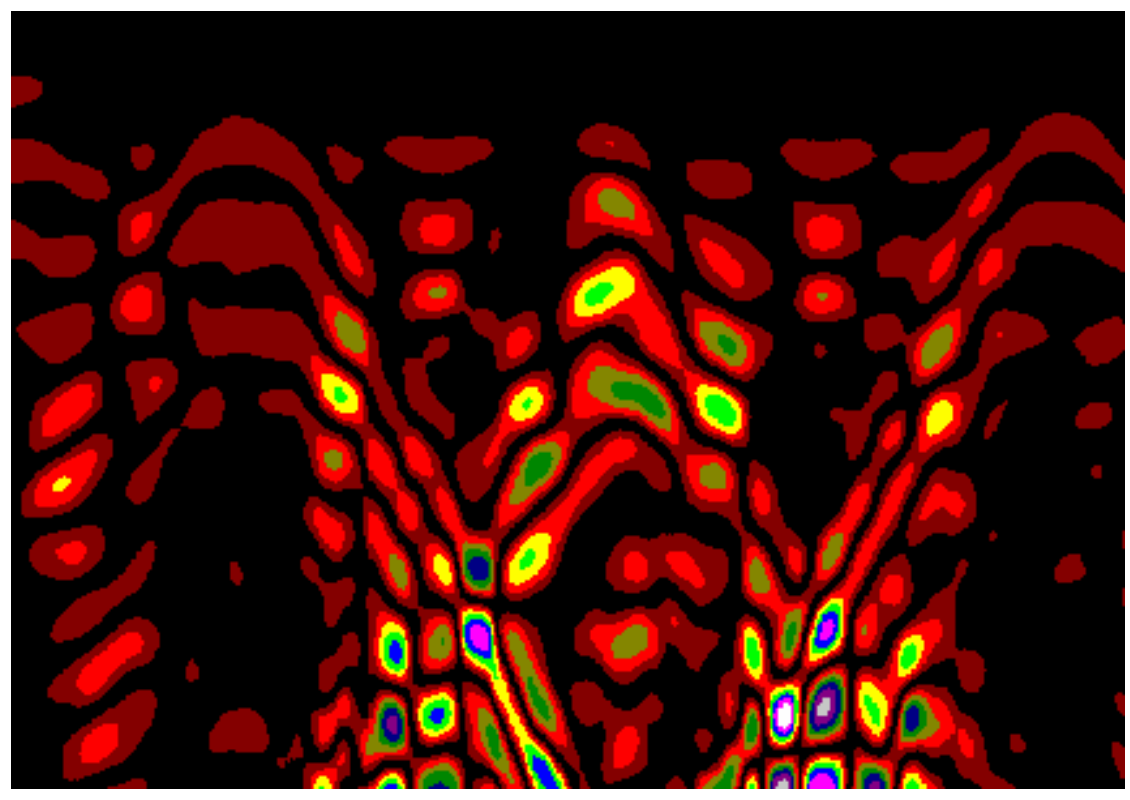

Figure 7.56: 2D linear scan of delamination embedded within top flange of FRP bridge deck module.

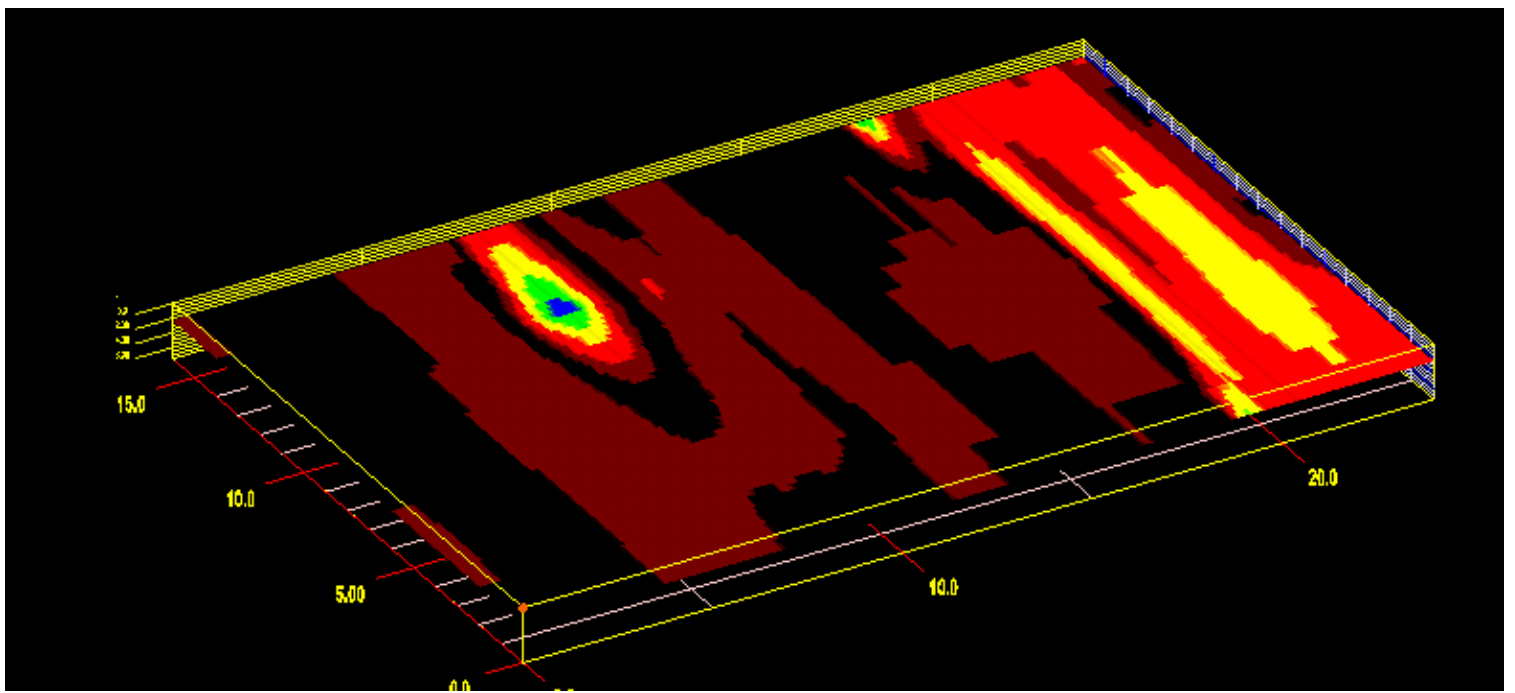

Figure 7.57: 3D map of delamination module constructed by the constant gain data set. 


\subsection{PARAMETER SETTINGS}

\subsubsection{Record Length (512 Points versus 1024 Points)}

The $1.5 \mathrm{GHz}$ GPR system is capable of acquiring data using different record lengths. Obviously, data collection using more points per waveform in any given scan will improve its resolution. Consequently, the speed at which 1024 data can be acquired will be slower than that of 512 points. Laboratory tests showed that data acquisition speed is not of real concern between 512 and 1024 point settings since they are relatively close to each other. However, further studies should be done to evaluate the importance of data acquisition speed in the field using 512 points versus 1024 points.

In terms of data quality, it is clear that 1024 point setting does provide a better resolution (Figure 7.58), which could be important for detecting defects with smaller sizes. As for defects with larger sizes, there is no distinct advantage of using 1024 points versus 512 points. Overall, a 1024 point data acquisition is recommended for subsurface defect detection in FRP bridge decks since this setting provides better resolution for smaller defect sizes.

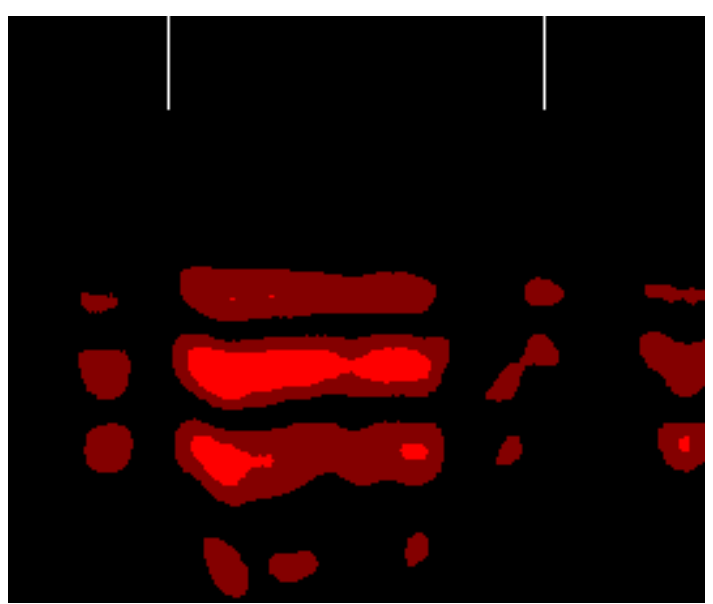

(a)

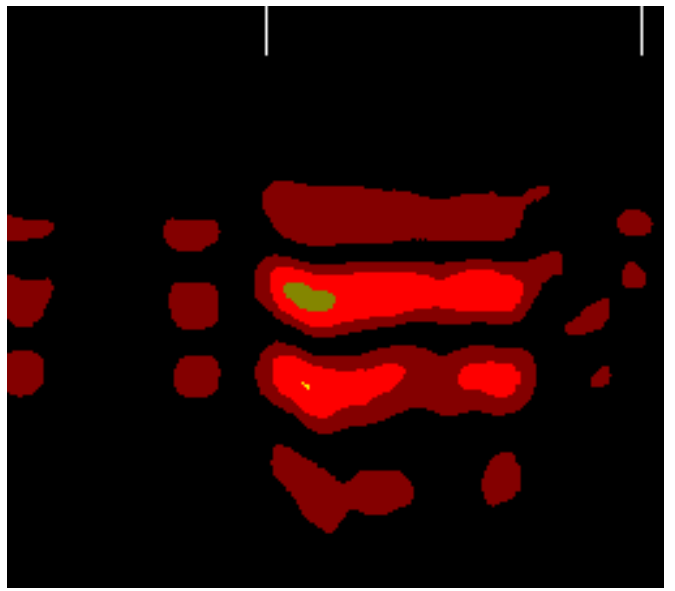

(b)

Figure 7.58: (a) 512 points 2D scan (b) 1024 points 2D scan. 


\subsubsection{Constant Gain versus Linear Gain}

Before the actual data acquisition process begins, gain is a function that could be applied to all the subsequent data collection. It is important to understand the dynamics of applying gain to a set of data. While some argue that the data could be collected first and a gain function (linear or constant) can be applied later during the post-processing, this laboratory study has found that the quality of data is far better if gain is applied during the data acquisition stage itself. Post-processing gain could again be applied to such data.

Figure 7.59 shows a 2D GPR scan obtained using a constant gain for a 10ns range data collection. It is evident from this figure that a constant gain is not sufficient in

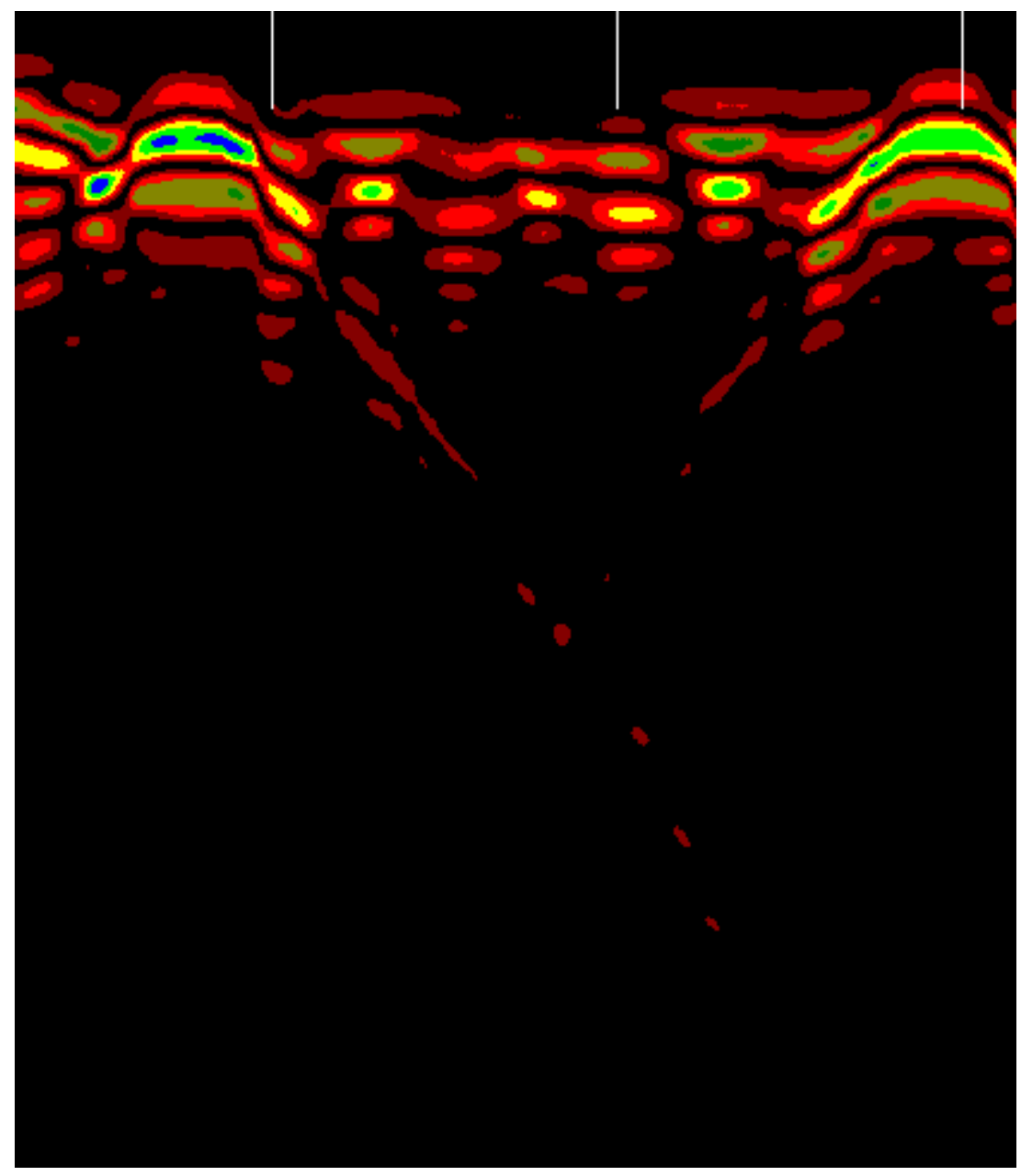

Figure 7.59: A constant gain 2D scan of 10ns range. 
recording vital information needed for defect detection in the bottom flange. In fact, no useful information can be obtained beyond $4 \mathrm{~ns}$ range.

Traditionally, the application of linear gain does not affect the quality of a top

flange 2D scan. However, when the defect size gets smaller, the magnified signal may interfere with the signal of the small defect thus affecting the detection of such defect. At the same time, a linearly (or exponentially) increasing gain is necessary for defect detection in the bottom flange.

The actual gain value that is required will vary from surface to surface, and it is also dependent on environmental parameters such as moisture content of the deck. It is strongly recommended that proper gain setting be calibrated upfront according to the equipment manufacturer's suggested procedure. Proper use of gain function allows for acquisition of better quality data, which will ensure a successful GPR test.

\subsection{BLIND TEST}

A full scale FRP bridge deck (3' x 6') was cast with an integral wearing surface. During casting, six water-filled pouches (frozen initially to facilitate easy placement) of various sizes and thicknesses were added between the wearing surface layer and the FRP bridge deck to simulate water-filled debonds. Figure 7.60 shows the defect layout. After the wearing surface was cured for 24 hours, a GPR test using $1.5 \mathrm{GHz}$ ground-coupled antenna was conducted while the water-filled debonds (now with liquid water) between the surfaces were still intact. Since this was a blind test, the deck specimen was prepared by someone else and the locations of the defects were not known to the GPR surveyor. 


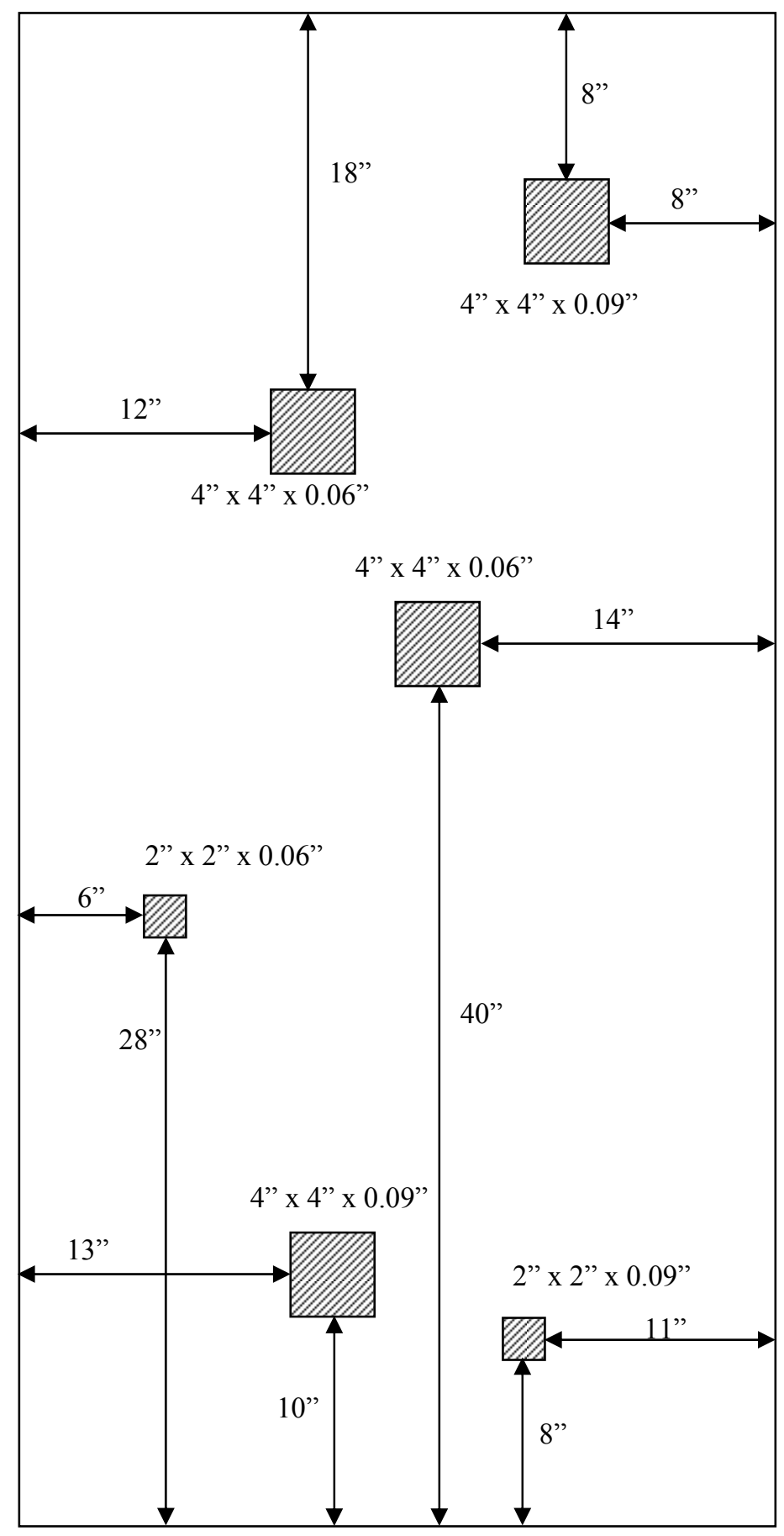

Figure 7.60: Defect layout for blind test. 


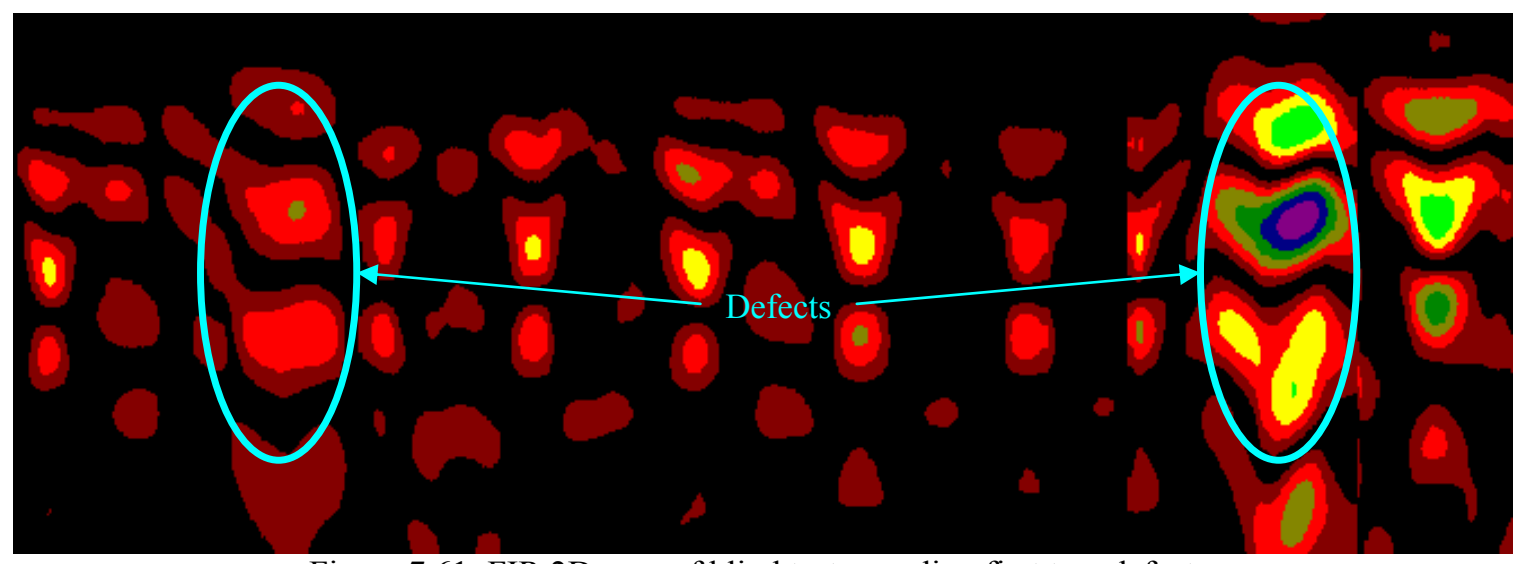

Figure 7.61: FIR 2D scan of blind test revealing first two defects.

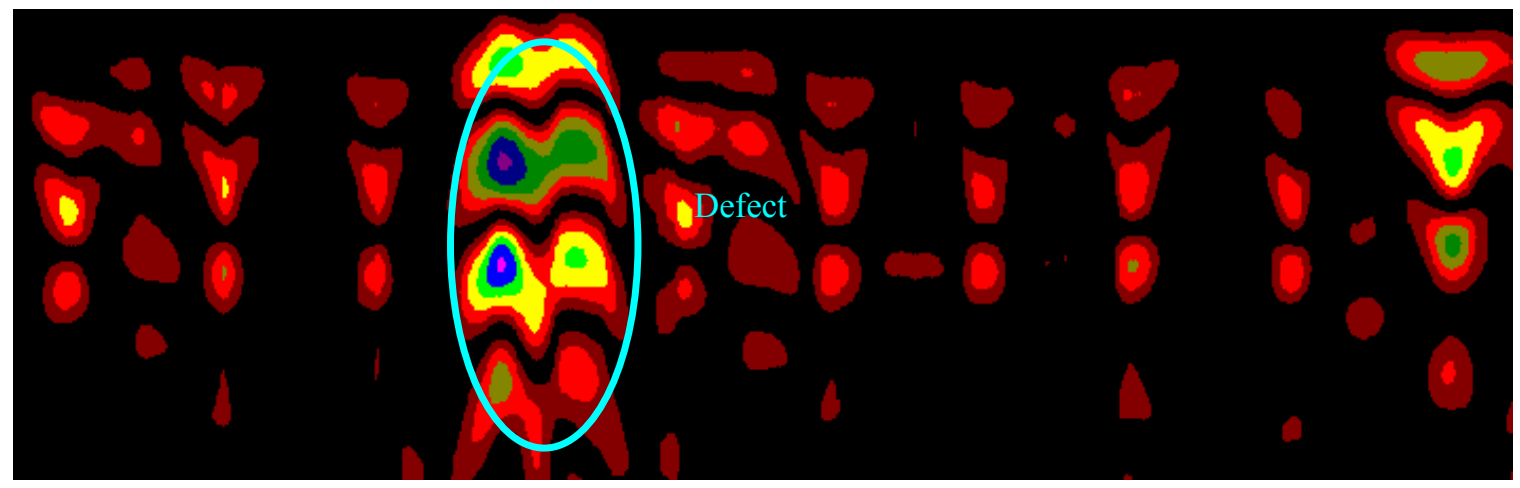

Figure 7.62: FIR 2D scan of blind test revealing third defect.

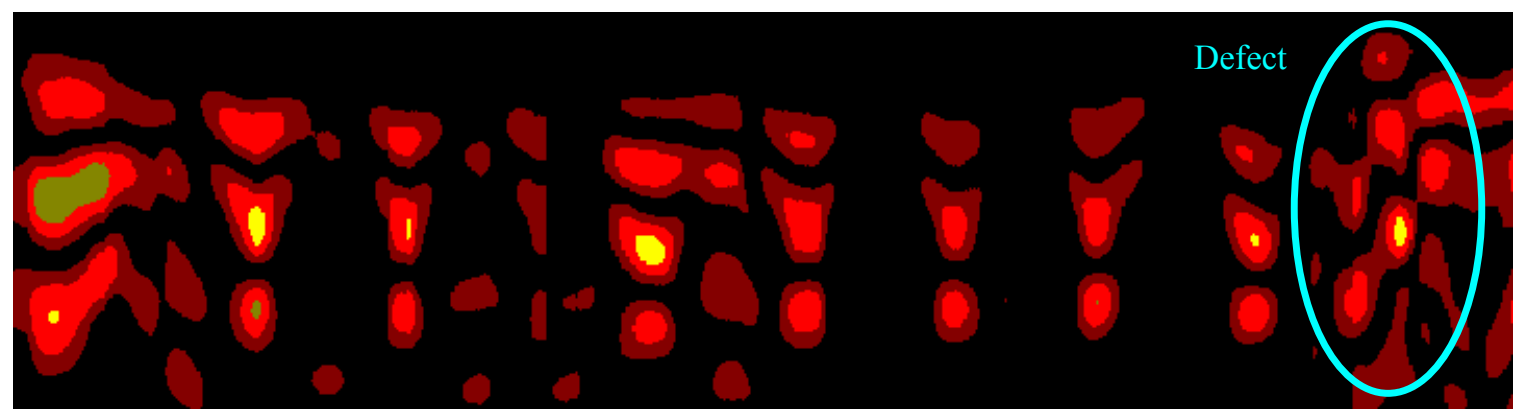

Figure 7.63: FIR 2D scan of blind test revealing the fourth defect.

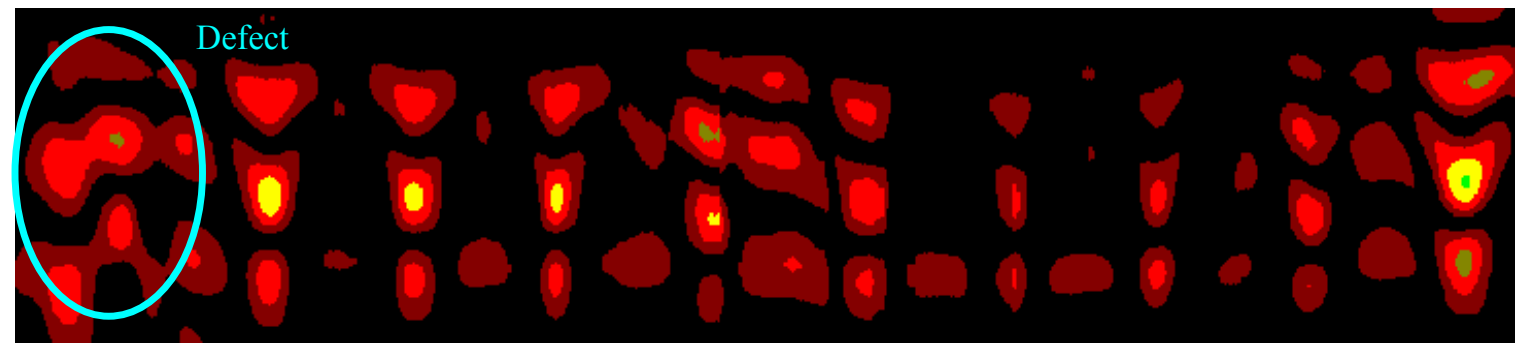

Figure 7.64: FIR 2D scan of blind test revealing the fifth defect. 
Eight longitudinal scans were collected at 4" spacing from right to left. The first scan was collected 4" away from the edge of the deck. From Figures 7.61 and 7.62, one can clearly identify 3 defects. Figure 7.63 shows the defect because of the disappearance of the high intensity spike near the end of the scan. In Figure 7.64, though there appears to be a defect, further testing was needed to confirm its existence. Besides, only 5 defects were found in the first round of testing, thus requiring a refined round of testing to identify the last defect. The first round of GPR testing was done with 4" scan spacing, thus there's a possibility that a smaller defect could go unnoticed due to $1.5 \mathrm{GHz}$ antenna's small footprint. Therefore, a total of seven additional scans were taken, each in between two of the first round scans. Ultimately, the entire blind test was conducted with a 2" spacing data collection.

In Figure 7.65 , the sixth defect can be seen clearly in the early part of the scan. The other defect shown towards the end of the scan is one that was identified before. Since it's showing up in both scans, it is a large 4" x 4" defect. In Figure 7.66, the 2D scan basically confirmed the fifth defect that was detected previously from Figure 7.64.

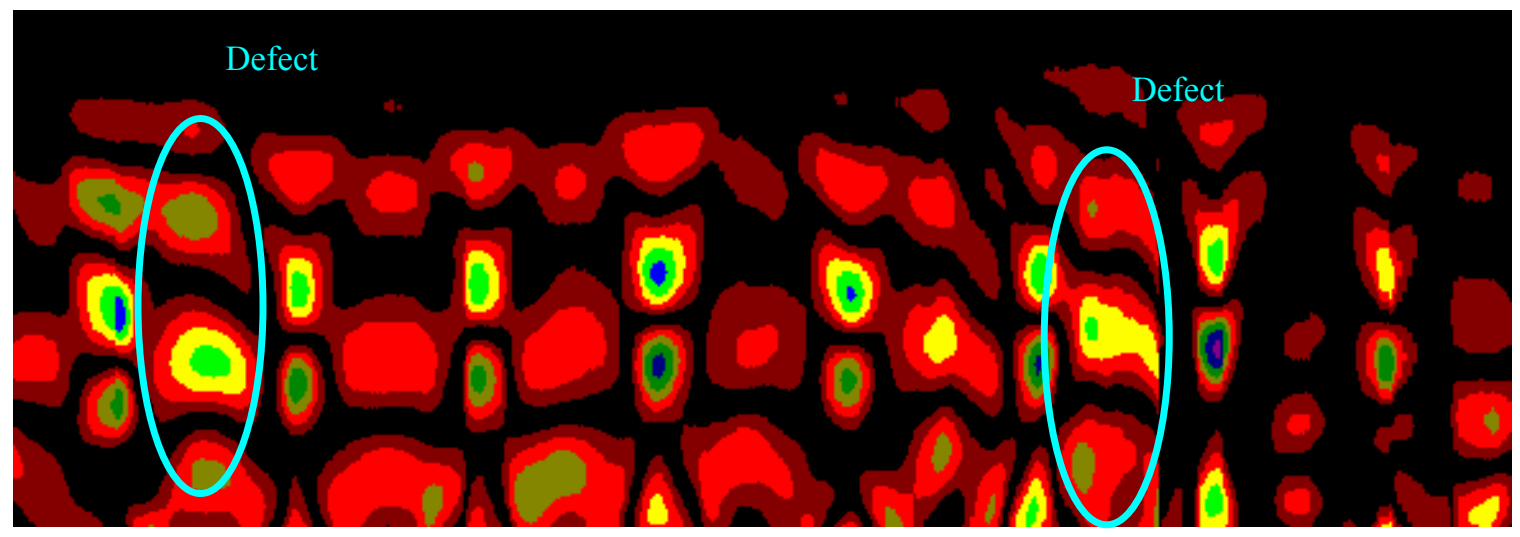

Figure 7.65: FIR 2D scan of blind test revealing the sixth defect. 


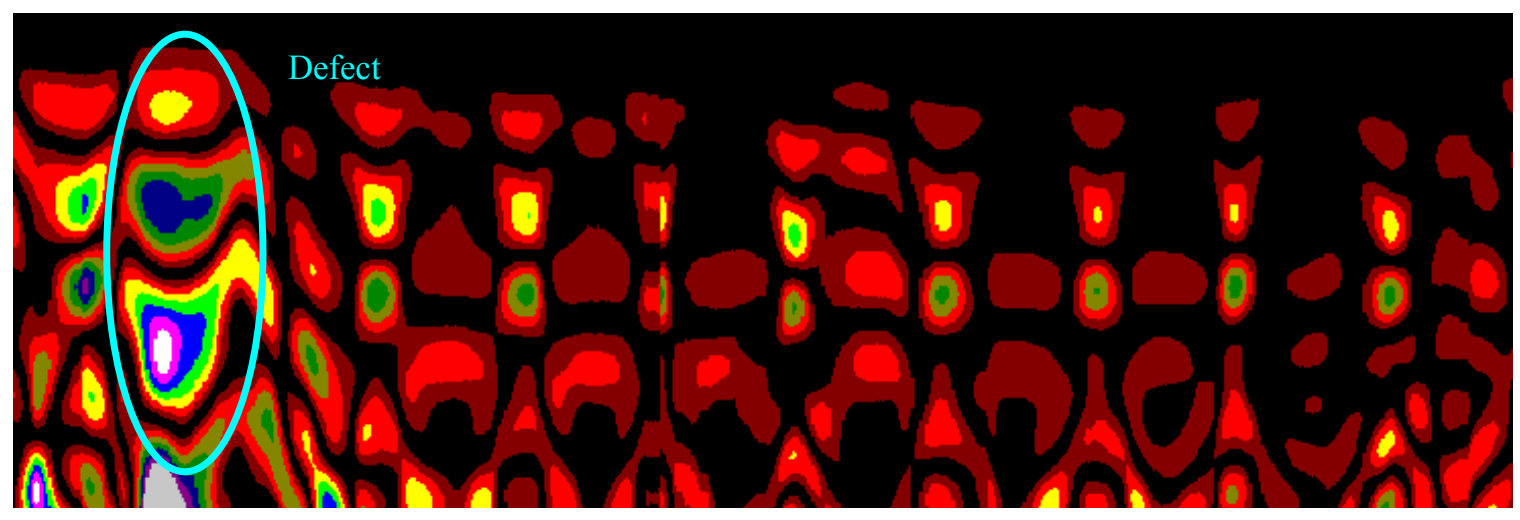

Figure 7.66: FIR 2D scan of blind test confirming the fifth defect shown in Figure 7.64.

Notice that GPR is excellent in detecting the water-filled debonds, but was unable to provide important information such as the planar size of the defects or its thickness. Future study should attempt to quantify the results in hope of categorizing the defects by type, size, and thickness.

Figure 7.67 shows the 3D plot of the defect location. The $1.5 \mathrm{GHz}$ ground-coupled antenna is proven to be extremely effective in detecting water-filled debond.

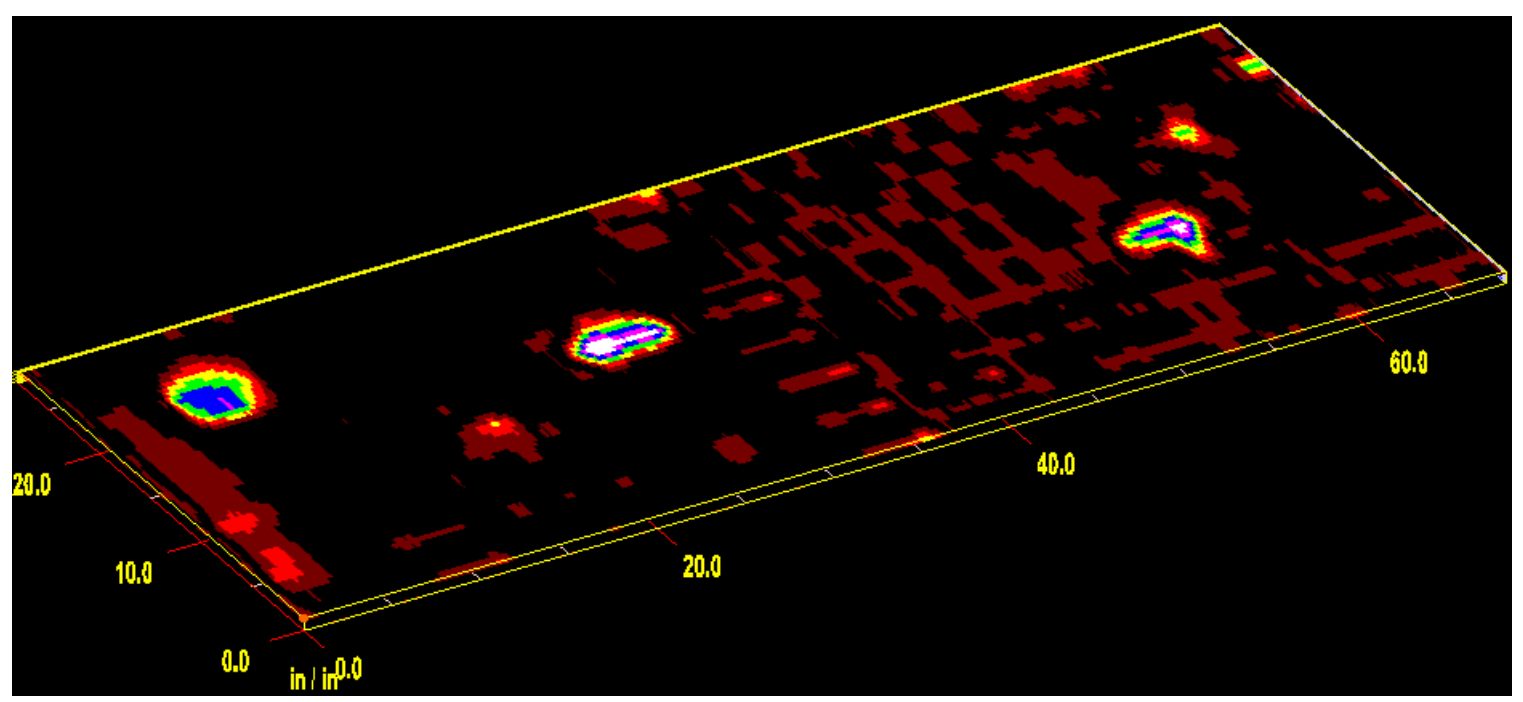

Figure 7.67: 3D map showing the location of the water-filled defects. 


\subsection{CONCLUSIONS}

Based on the post processing results, it is concluded that $2.0 \mathrm{GHz}$ horn antenna GPR system is not capable of detecting air-filled defects regardless of size and thickness. This is consistent with the general consensus regarding the low effectiveness of any GPR system for air-filled defects. By combining the 2D images, 3D images, and signal analysis of both original data and data sets applied with Finite Impulse Response (FIR), the smallest water-filled defect that could be detected by the $2.0 \mathrm{GHz}$ air-coupled horn antenna was found to be $8 " \times$ × 8 " $0.06 "$.

On the other hand, the $1.5 \mathrm{GHz}$ ground-coupled antenna proved to be an excellent tool in detecting subsurface water-filled defects (both debonds and delaminations). The smallest water-filled debond that could be detected was 2" 2 2" $\mathrm{x} 0.06$ " given the footprint size of the $1.5 \mathrm{GHz}$ antenna. Although it has a 1 " footprint size on each side of the center line, the signal decreases drastically. Hence, in order to detect a very small defect, such as a 1" x 1" debond, the antenna has to go directly over the defect. Not only is the probability of achieving such feat a monumental task in the field, it is simply not practical considering the large size of the area usually surveyed using GPR system.

In order to conduct a successful GPR test, a time range of $4 \mathrm{~ns}$ is recommended for defect detection in the top flange while 10ns range should be used for the bottom flange. Furthermore, in conjunction with 10ns range, a linearly increasing gain is needed to magnify the echo signals from the bottom flange and produce meaningful results. Laboratory test did not find any advantage in using 512 point record length over 1024 points in terms of acquisition speed. However, the 1024 point record length provided somewhat better resolution. Thus it is recommended that any GPR tests on FRP bridge decks be conducted using 1024 points record length.

The blind test conducted in this study proves that $1.5 \mathrm{GHz}$ can easily detect waterfilled debonds as small as 2 " x 2 " x $0.06 "$. 


\section{Chapter 8}

\section{CONCLUSIONS AND RECOMMENDATIONS}

\subsection{CONCLUSIONS}

In summary, results have shown that while commercially available heaters are capable of finding defects as small as 1 " x 1 " $(25.4 \mathrm{~mm} \times 25.4 \mathrm{~mm})$ in laboratory setting, it is certainly not as good at detecting defects in a larger scale specimen since the smallest air-filled defect that can be detected was 2" x 2" x 0.06 " $(50.8 \mathrm{~mm} \times 50.8 \mathrm{~mm} \times 1.5 \mathrm{~mm})$. This is because moving the heater back and forth in larger specimen reduces the heat intensity. This problem can be addressed using multiple heaters, or by testing larger areas in small pieces. Furthermore, use of heaters was not found to be very effective in detecting water-filled defects.

Solar radiation can be a good heating source if used properly. The best times to detect air-filled debonds are between 12 noon to 5 PM. Night time may provide better result for IRT testing if an external heating source is going to be employed since the temperature fluctuation is relatively low at night. A relatively low wind velocity environment is needed to produce good results. The solar radiation tests have shown that 4" x 4" x $0.09 "(101.6 \mathrm{~mm} \times 101.6 \mathrm{~mm}$ x $2.25 \mathrm{~mm})$ water-filled debond can be detected on a warm sunny day (for example, during the summer months), while the ability to detect smaller and thinner water-filled debonds need further improvement. Also, solar tests have shown that all the $4 " \mathrm{x} 4 "(101.6 \mathrm{~mm} \times 101.6 \mathrm{~mm})$ air-filled debonds as thin as 0.06 " $(1.5 \mathrm{~mm})$ can be detected in warm September weather and colder March days using solar energy as primary heat source. The results also showed that the defect clarity in colder 
March days was not very good but the warm September day produced good results. The test also showed that delamination within the flanges of FRP decks cannot be detected using only solar radiation.

The heating blankets proved to be the weakest active heat source explored in this study. It was not capable of detecting any debonds (air-filled and water-filled) smaller than $8 " \mathrm{x} 8$ " in size. Although the heating blankets were sending out $1200 \mathrm{~W} / \mathrm{m}^{2}$ of output (roughly the same amount of energy emitted by the sun on a hot sunny day in June), it did not perform as well as the solar radiation test because the specimens were heated by the sun for a much longer period of time compared to only 4 minutes using the heating blanket. When comparing the heating blanket to the heaters, it is clear that heaters provide much greater heat intensity $\left(\cong 2500 \mathrm{~W} / \mathrm{m}^{2}\right.$ output) for IRT. The only setback regarding the heaters is its inability to apply uniform heat to a large area while the heating blankets provided uniform heating of the specimens.

Based on the comparison between the actual laboratory data and the theoretical data generated through the use of finite element model, it is clear that the FE model has an acceptable accuracy in describing the actual heat transfer process between an air-filled debond and the surrounding. Future studies should focus on improving the modeling around the boundary of the debond to better describe the actual heat transfer in the vicinity of an air-filled debond.

The FE model for fully water-filled debond has shown promising characteristics. Unfortunately, adequate experimental data is currently unavailable to support the validity of the model. Future testing of IRT on water-filled debond should be done to provide data for verification purposes. 
Since the results have shown that the FE predictions for partially water-filled debond case are questionable, future effort should focus on successfully modeling such cases since it may be more representative of the field conditions.

Based on the post processing results, it is concluded that $2.0 \mathrm{GHz}$ horn antenna GPR system is not capable of detecting air-filled defects regardless of size and thickness. This is consistent with the general consensus regarding the lack of effectiveness of any GPR system in detecting air-filled defects. By combining the 2D images, 3D images, and signal analysis from both original data and data sets applied with Finite Impulse Response (FIR), the smallest water-filled defect that can be detected by the $2.0 \mathrm{GHz}$ airlaunched horn antenna is $8 " \mathrm{x} 8$ " $\mathrm{x} 0.06 "$.

On the other hand, the $1.5 \mathrm{GHz}$ ground-coupled antenna proved to be an excellent tool in detecting subsurface water-filled defects (debond and delamination). The smallest water-filled debond that can be detected is 2 " $\mathrm{x} 2$ " $\mathrm{x} 0.06$ ". While results certainly indicated the ability to detect even smaller defects using $1.5 \mathrm{GHz}$ ground-coupled antenna, the problem remains due to footprint size of the $1.5 \mathrm{GHz}$ antenna. Although it has a 1" footprint to each side from the center line, the signal decreases drastically away from the center line. Hence, in order to detect a small defect, such as 1" x 1" debond, the antenna has to go directly over the defect. Not only is the probability of achieving such feat a monumental task in the field, it is simply not practical considering the large size of the areas usually surveyed by the GPR system.

In summary, the $1.5 \mathrm{GHz}$ ground-coupled antenna has out-performed the $2.0 \mathrm{GHz}$ air-coupled horn antenna in subsurface defect detection for FRP bridge decks. 
In order to conduct a successful GPR test, a time range of $4 \mathrm{~ns}$ is recommended for defect detection in the top flange while 10ns should be used for the bottom flange. Furthermore, in conjunction with 10ns range, a linear gain is needed to magnify the signals from the bottom flange of the FRP deck to produce meaningful results. Laboratory test did not find any major advantage in terms of data acquisition speed in using 512 points record length over 1024 points. However, the results clearly show that 1024 point data provided somewhat better resolution. Thus it is recommended that GPR tests on FRP decks be conducted using 1024 points record length. The blind test results proved that $1.5 \mathrm{GHz}$ antenna can detect water-filled debonds as small as 2 " x 2 " x 0.06 ".

Finally, it has proved to be beneficial to combine IRT's ability to detect air-filled debonds if a proper active heat source is employed and GPR's ability to detect waterfilled defects. GPR can even detect delaminations in the bottom flange of an FRP bridge deck for a thorough nondestructive evaluation. By complementing each technique's short-coming, the combination of IRT and GPR will greatly improve the quality of nondestructive evaluation and associated maintenance plan for FRP bridge decks, which is necessary to ensure their long-term in-service performance.

\subsection{RECOMMENDATIONS FOR FUTURE RESEARCH}

It is recommended that future research be focused on quantitative analysis of GPR data for extracting vital information such as defect thickness. Such quantitative models will also be needed for automating the signal analysis procedure. As for IRT, a more powerful yet efficient heat source should be explored for field use. Also, quantitative analysis of IRT data should be explored to improve IRT's performance in defect detection. 


\section{REFERENCES}

Barnes, C. L., and Trottier, J. F. (1999). "Use of Ground Penetrating Radar for Estimating Deterioration Repair Quantities on Reinforced Concrete Bridge Decks.” Department of Civil Engineering, Dalhousie University, Daltech Halifax, Nova Scotia, Canada. World Wide Web publication.

http:/www.dal.ca/ barnesc/Quebec.html

Brown, J. R. and Hamilton III, H. R. (2003). "NDE Reinforced Concrete Strengthened with Fiber-Reinforced Polymer Composites using Infrared Thermography." Proceedings of the InfraMation 2003, Las Vegas, NV, October 13-16.

Carter, C. R., Chung, T., Reel, R., Tharmabala, T., and Wood, D. (1995). “Impulse Radar Evaluation of Reinforced Concrete Bridge Decks." Society of Photo-Optical Instrumentation Engineers (SPIE), Vol. 2456, pp. 174-184.

Cook, J. C. (1960). "Proposed Monocycle-Pulse, VHF Radar for Airborne Ice and Snow Measurements.” AIEE Trans. Commun. And Electron., 79(2), 588-594.

FLIR SYSTEMS (2002a). ThermaCAM ${ }^{T M}$ S60 Operator's Manual, Revision A, 14 June.

FLIR SYSTEMS (2002b). ThermaCAM ${ }^{T M}$ Researcher Operating Manual, Version A.

Groenenboom, J., van der Kruk, J. and Zeeman, J. H. (2001). “3D GPR Data Acquisition and the Influence of Positioning Errors on Image Quality." Extended abstract EAGE, $63^{\text {rd }}$ Conference and Technical Exhibition, Amsterdam, Netherland. June, 2001.

Groenenboom, J. and Yaravoy, A. G. (2000) "Data Processing for A Landmine Detection Dedicated to GPR." Proceedings of the Eight International Conference on Ground Penetrating Radar, May, 2000. 
Halabe, U. B., Vasudevan, A., GangaRao, H. V. S., Klinkhachorn, P., and Lonkar, G. (2004a). "Subsurface Defect Detection in FRP Composites Using Infrared Thermography." Proceedings of the Thirty-First Annual Review of Progress in Quantitative Nondestructive Evaluation, Vol. 24 (American Institute of Physics - Vol. CP760), Colorado School of Mines, Golden, Colorado, July 25 - 30, pp. 1477-1484.

Halabe, U. B., Vasudevan, A., Roy, M., and GangaRao, H. V. S. (2004b). "Infrared Thermography for Detecting Subsurface Debonds and Delaminations in FRP Bridge Decks Subjected to Accelerated Aging." ASNT Fall Conference and Quality Testing Show - 2004, Paper Summaries, Las Vegas, NV, November 15-19, pp. 129-131.

Halabe, U. B., Vasudevan, A., and GangaRao, H. V. S. (2004c). "Infrared Thermography for Monitoring Subsurface Debonds in FRP Bridge Decks Subjected to Fatigue Loading." ASNT Fall Conference and Quality Testing Show - 2004, Paper Summaries, Las Vegas, NV, November 15-19, pp. 132-134.

Halabe, U. B., Vasudevan, A., Klinkhachorn, P., and Lonkar, G. (2004d). "Task 5.2.1.: Develop an Infrared Thermography Based System to Detect Delaminations.” FHWA Contract No. DTFH61-01-C-00002, Draft Final Report, submitted to Federal Highway Administration (FHWA), Washington, D.C., April, 183 pp.

Halabe, U. B., Vasudevan, A., and GangaRao, H. V. S. (2003a). "Nondestructive Testing of Composite Bridge Components Using Digital Infrared Thermography." ASNT Fall Conference and Quality Testing Show - 2003, Paper Summaries, Pittsburgh, PA, October 13-17, 2003, pp. 120-122.

Halabe, U. B., Bangalore, G., GangaRao, H. V. S., and Klinkhachorn, P. (2002). "Infrared Scanning of FRP Composite Members." Proceedings of the Twenty-Ninth Annual Review of Progress in Quantitative Nondestructive Evaluation, Vol. 22, Bellingham, Washington, July 14 - 19, pp. 1003-1010. 
Halabe, U. B., Maser, K. R., and Kausel, E. A. (1995). "Condition Assessment of Reinforced Concrete Structures Using Electromagnetic Waves." American Concrete Institute (ACI) Materials Journal, Vol. 92, No. 5, pp. 511-523.

Halabe, U. B. (1990). "Condition Assessment of Reinforced Concrete Structures Using Electromagnetic Waves." Ph.D. Dissertation, Department of Civil Engineering, Massachusetts Institute of Technology (MIT), Cambridge, MA.

Halabe, U. B., and Maser, K. R. (1986). “Thermal Modeling of A Leaky Roof.” MIT CCRE-Technical Note 86-4, Center for Construction Research and Education, Department of Civil and Environmental Engineering, Massachusetts Institute of Technology, Cambridge, MA.

Hodge Jr., S. (1987). Thermography \& Personal Injury Litigation. Wiley Law Publications, pg. 71.

Jackson, D. R., Islam, M., and Alampalli, S. (2000). "Feasibility of Evaluating the Performance of Fiber Reinforced Plastic (FRP) Wrapped Reinforced Concrete Columns Using Ground Penetrating Radar (GPR), and Infrared (IR) Thermography Techniques." Proceedings of Structural Materials Technology IV - An NDT Conference, Atlantic City, NJ, February 28 - March 3.

Johnson, E.C., Nokes, J. P., and Hawkins, G. F. (1999). "NDE of Composite Seismic Retrofits to Bridges." Nondestructive Characterization of Materials IX, AIP conference proceedings 497, Sydney, Australia, p. 367-372.

Kaplan, H. (1999). Practical Applications of Infrared Thermal Sensors and Imaging Equipment, SPIE - The International Society for Optical Engineering, Bellingham, Washington, USA. 
Maldague, X. P. V., and Moore, P. O. (2001). "Infrared and Thermal Testing."

Nondestructive Testing Handbook. American Society for Nondestructive Testing, p. 3.

Maldague, X. (1993). Nondestructive Evaluation of Materials by Infrared Thermography, Springer-Verlag, London.

Malloy, J.F. (1969). Thermal Insulation. Reinhold Book Corporation, New York, NY.

Maser, K. R., Horschel, M. G., and Grivas, D. A. (2002a). "Integration of Ground Penetrating Radar and Infrared Thermography for Bridge Deck Assessment." Proceedings of Structural Materials Technology V-An NDT Conference, Cincinnati, OH, September 10-13, pp. 119-128.

Maser, K. R., Roberts, R., Kutrubes, D., and Holland, J. (2002b). "Technology for Quality Assurance of New Pavement Thickness." Proceedings of Structural Materials Technology V-An NDT Conference, Cincinnati, OH, September 10-13, pp. 217-226.

Maser, K. R., and Roddis, W. M. K. (1990). "Principles of Thermography and Radar For Bridge Deck Assessment." Journal of Transportation Engineering, 116(5), SeptemberOctober.

Miceli, M., Horne, M. R., and Duke, J. C. (2001). "Health Monitoring of FRP Bridge Decks.” Advanced Nondestructive Evaluation for Structural and Biological Health Monitoring, Proceedings of SPIE, 4335, March 6-8, 100-105.

Moffatt, D. L., and Puskar, R. J. (1976). “A Subsurface Electromagnetic Pulse Radar.” Geophysics, 41(3), 506-518.

Mtenga, P.V., Parzych, J.G., and Limerick, R. (2001). "Quality Assurance of FRP Retrofit Using Infrared Thermography.” Proceedings of the 2001 Structural Congress and Exposition, Washington, D.C., May 21-23. 
Mutnuri, B. (2005). "Thermal Conductivity Characterization of Composite Materials." Master Thesis, Department of Mechanical Engineering, West Virginia University, Morgantown, WV.

Neville, A.M. (1973). Properties of Concrete. John Wiley \& Sons, Inc., New York, NY.

Radzevicius, S., and Daniels, J. (2000). "Ground Penetrating Radar Polarization and Scattering From Cylinders.” Journal of Applied Geophysics, 45, 111-125.

Olhoeft, G. R. GEORADAR: Ground Penetrating Radar. World Wide Web. http://www.g-p-r.com/

Reynolds, W.N., and Wells, G.M. (1984). Video-compatible thermography. British Journal of NDT, January, p. 40-44.

Ring, E.F.J. (1997). Royal National Hospital for Rheumatic Diseases, Bath, England. University of Glamorgan - Inaugural Lecture Jan. $28^{\text {th }}$.

Shepard, S. M., Rubadeux, B. A., and Ahmed, T. (1999). "Automated Thermographic Defect Recognition and Measurement." Nondestructive Characterization of Materials IX, AIP Conference Proceedings 497, Sydney, Australia, p. 373-378.

Smemoe, C.M. (2000). "Processing and Visualization of Ground Penetrating Radar Data for Assessing Natural Hydrogeologic Conditions." World Wide Web publication. http://emrl.byu.edu/chris/gpr.htm\#_Toc500291468

Touloukian, Y.S., and DeWitt, D.P. (1970). Thermal Radiative Properties. Thermophysical Properties of Matter, vol. 7, IFI/Plenum, New York, Washington. 
Tretout, H. (1987). “Applications Industrielle De La Thermographie Infrarouge Au Controle Non Destructif De Pieces En Materiaux Composites." Rev. Gen. Therm. Fr., vol. 301, p. 47- 53.

Van der Kruk, J. (2001). "Use of Crossed Dipole Antennas in 3D Imaging of MultiComponent GPR Data." Extended abstract SEG, $71^{\text {st }}$ Conference and Technical Exhibition, San Antonio, Texas. September, 2001.

Van der Kruk, J., Wapenaar, C., and Fokkema, J. (2001). "Comparison of Resolution Functions of 3D Multi-Component with 3D Single-Component Imaging Algorithms for Ground Penetrating Radar Data." Proceedings of $5^{\text {th }}$ SEGJ International Symposium Imaging Technology - The Society of Exploration Geophysicists of Japan (SEGJ), Japan, 229-236.

Vavilov (1980). "Infra-Red Non-Destructive Testing of Bonded Structures: Aspects of Theory and Practice.” British Journal of NDT, July, p. 175-183.

Vanzetti, R. (1972). Practical Applications of Infrared Techniques. John Wiley \& Sons, Inc., New York, NY.

Wang, T. and Oristaglio, M. (2000). "GPR Imaging using The Generalized Radon Transform.” Geophysics, 65, no. 5, 1553-1559. 


\section{APPENDIX A \\ RADAN POST PROCESSING}

\section{A.1 FINITE IMPULSE RESPONSE (FIR)}

Finite impulse response (FIR) is a built-in function within the GPR RADAN software. Its main function is to eliminate any constant noise generated by the GPR system during the data acquisition phase. FIR process is highly recommended for any data sets to be used for 3D modeling, since it would eliminate many unnecessary features within a GPR image (2D and 3D). The process of applying FIR filter to each set of data is described in detail below.

STEP 1: Open any data file that wished to be FIR filtered (Figure A1).

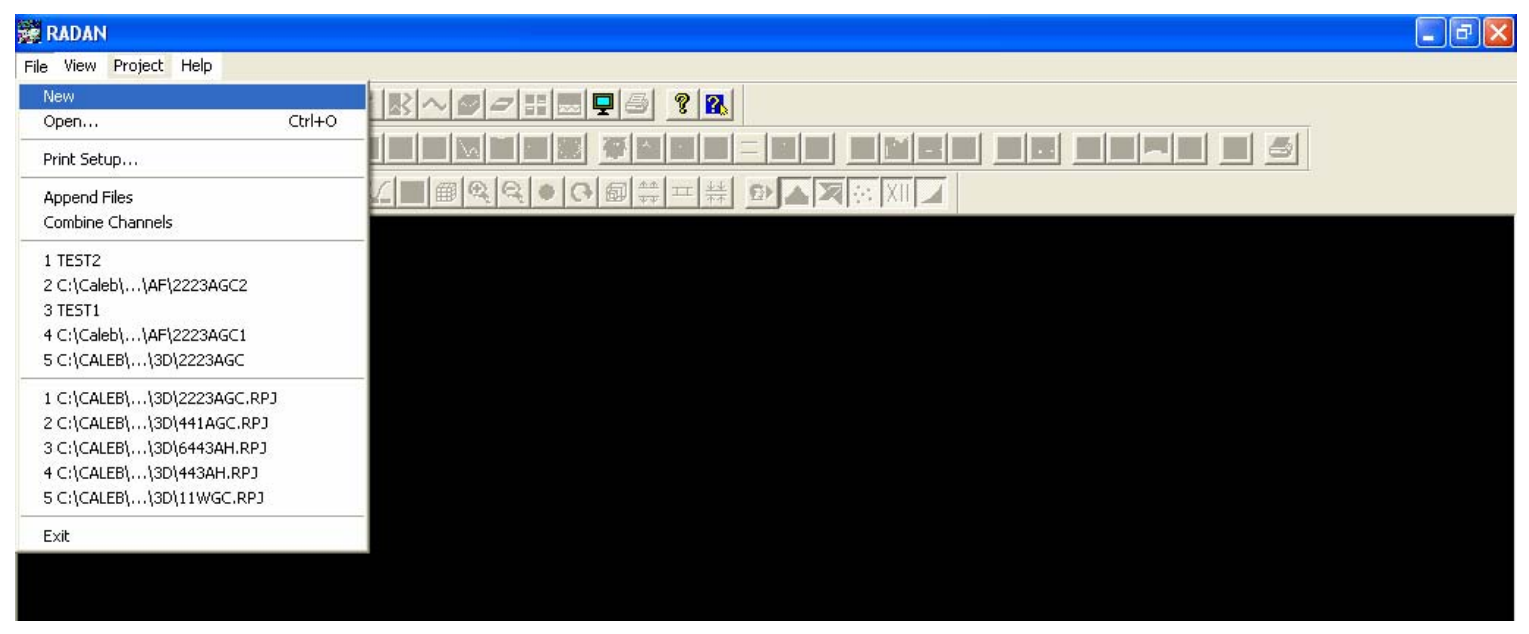

Figure A1: RADAN main operating screen.

STEP 2: Click on FIR Filter under the "Process" tab (Figure A2), or simply click on the

FIR icon 
STEP 3: On the ensuing screen, information pertaining to the data acquisition as well as the antenna is needed. Under the "Background Removal" column, type in a number equal to 1 less than the number of data acquisition point. For example, if the data was acquired using 512 points setting, type 511 in the "Background Removal" column. Under the "Vertical Filter" column, the "Low Pass" is equals to double of your antenna's frequency. For instance, if a $1.5 \mathrm{GHz}(=1500 \mathrm{MHz})$ antenna was used for data acquisition, then the number would be $1500 \times 2=3000$. Likewise, the high pass is usually $1 / 3$ of the antenna frequency. In this example, $500(1 / 3 \times 1500)$ was used. Finally, click "Apply" for the changes to take place, and then click "OK” (Figure A3).

STEP 4: Once the filter is applied, a screen will prompt for a location where the software will save the file.

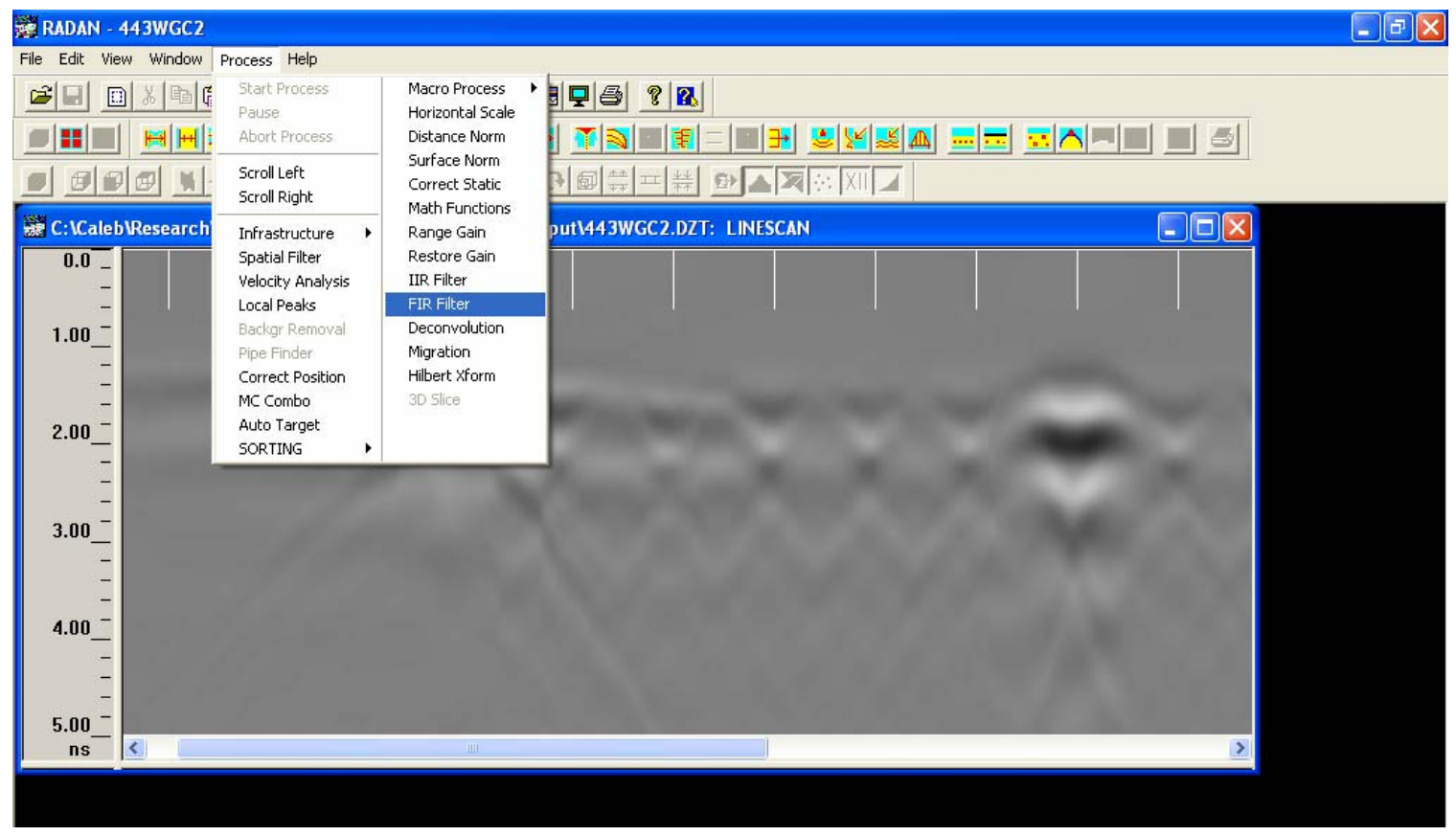

Figure A2: Click to apply FIR filter. 


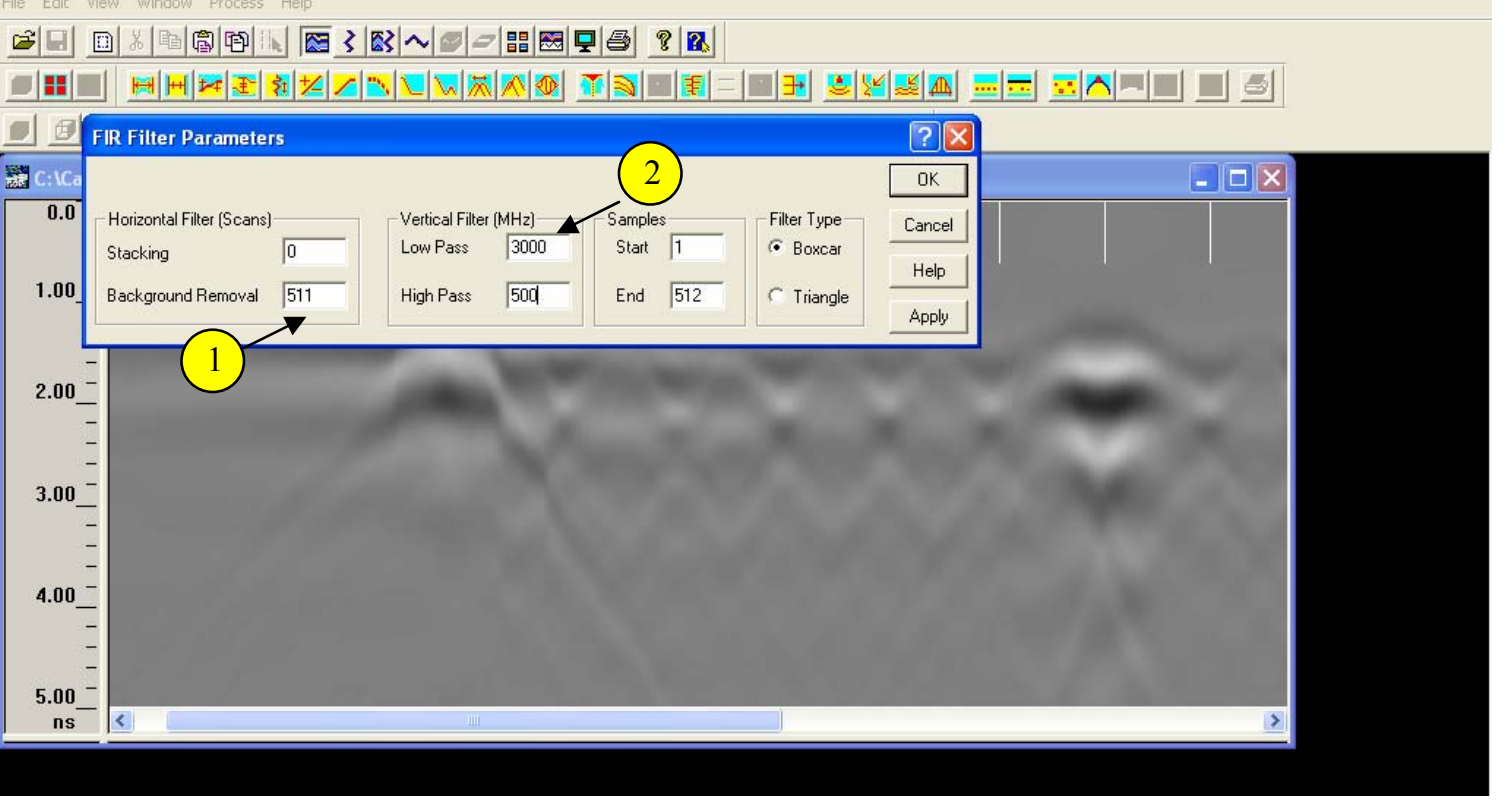

Figure A3: Input necessary information for FIR filter application.

\section{A.2 3D MODELING}

3D modeling enables the user to visually identify the defects and its locations. FIR filtered data are recommended for construction of 3D model since it will help the user by removing any unwanted noise and features within the 3D model. The process of $3 \mathrm{D}$ construction is described in detail below.

STEP 1: Start a new project by click "New" under the "File" tab.

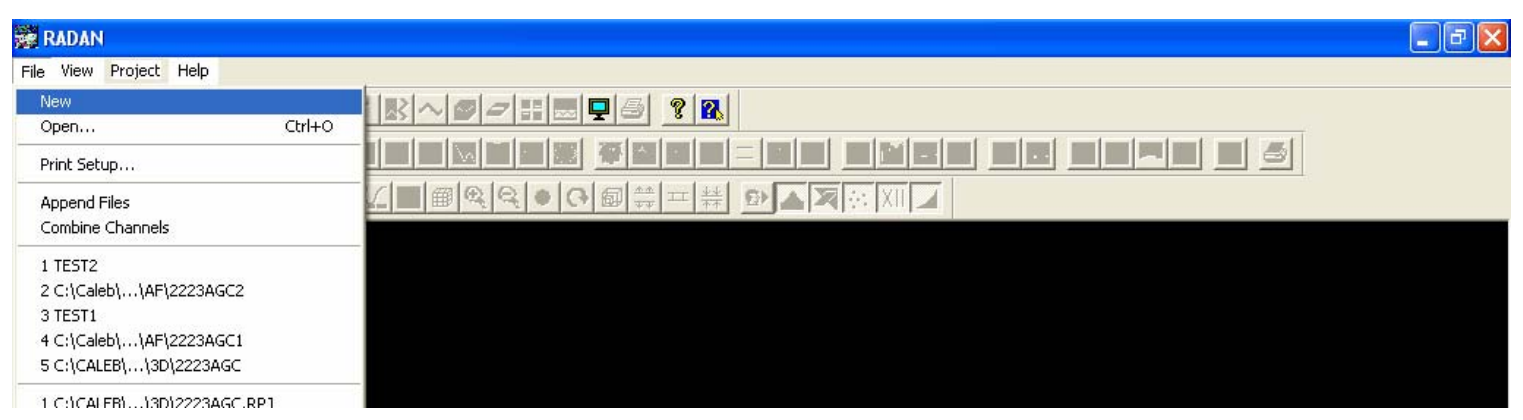

Figure A4: Start a new project for 3D construction. 
STEP 2: Provide a name for the 3D project, and check "RADBridge Project".

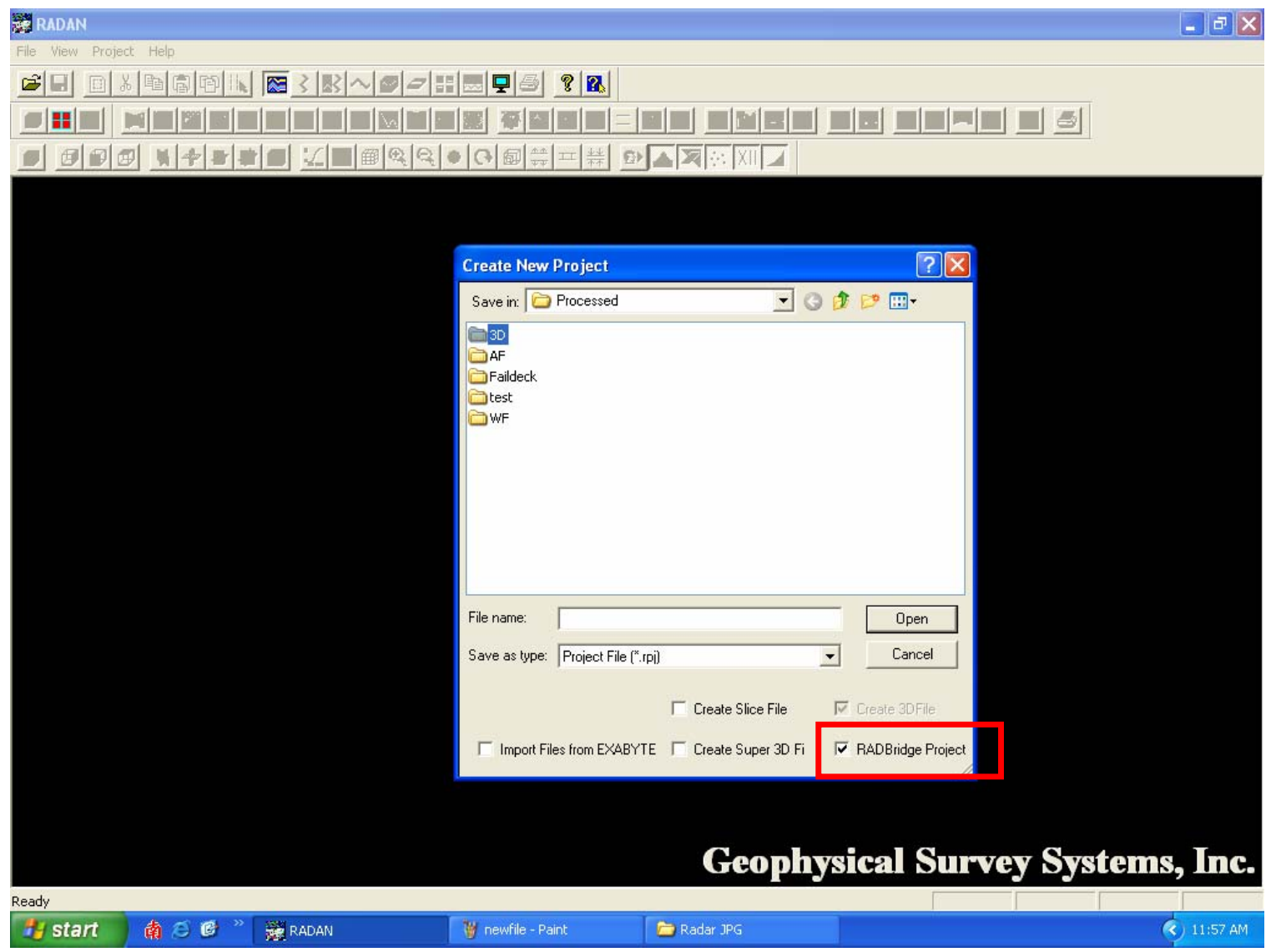

Figure A5: Create a new RADAN bridge project.

STEP 3: After providing the file name and the location where the 3D file is going to be saved, a list of required files need to be added one by one, starting from the file collected on the far right of the surveyed area and proceed left (Figure A6). When the addition is over, click "Done".

STEP 4: A dummy macro is needed to proceed. A dummy macro is a macro which multiplies the entire file set by 1 (essentially, not doing anything to the file set). This macro can be created once and re-used forever. Select the files attached before and click “Attach Macro” (Figure A7). 


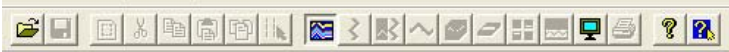

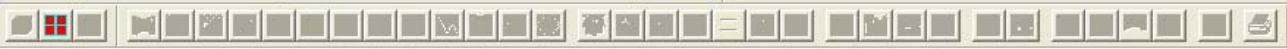

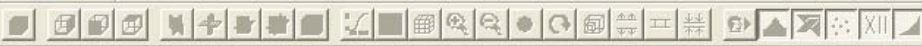

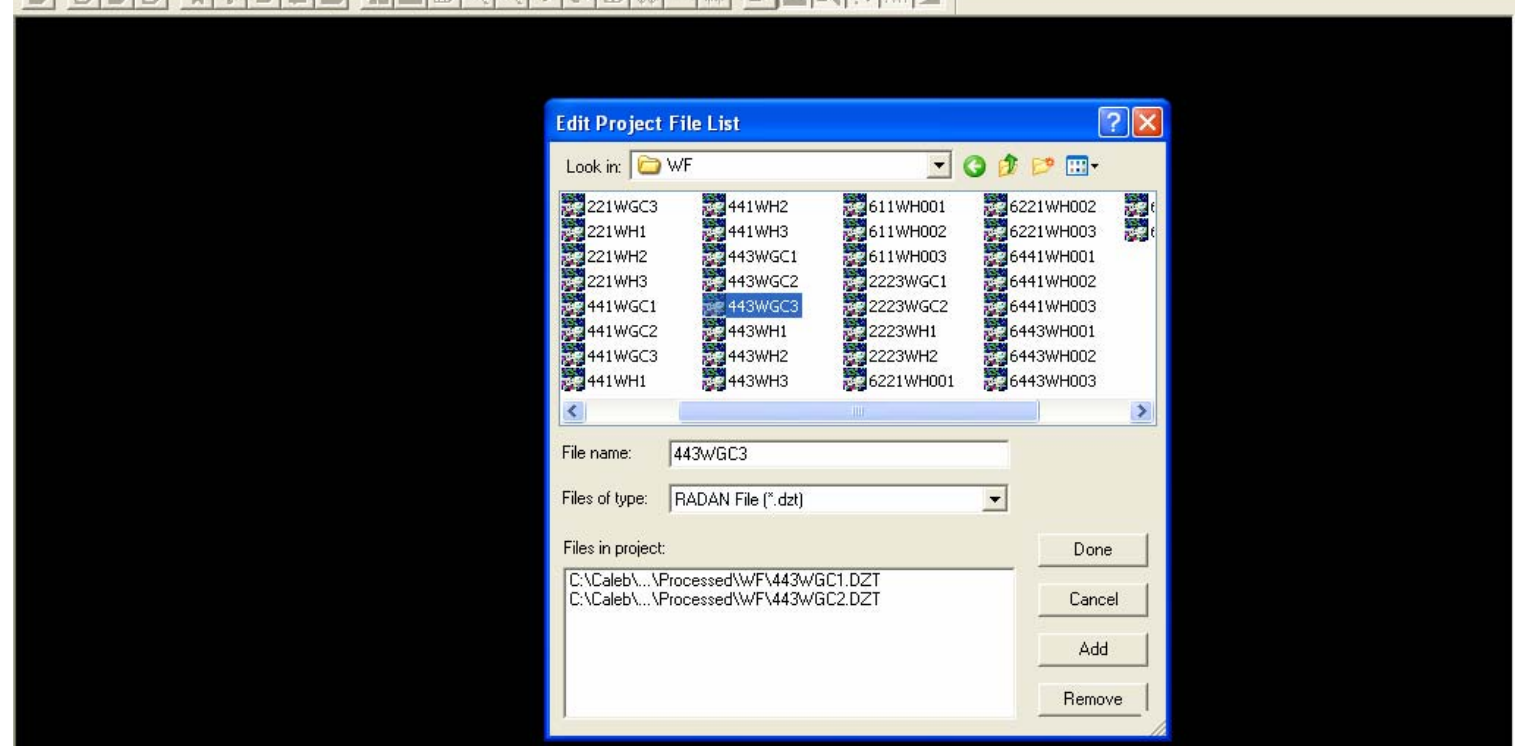

Figure A6: Adding necessary files for 3D construction.

TRADAN

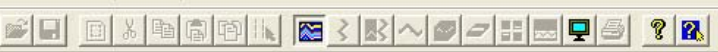

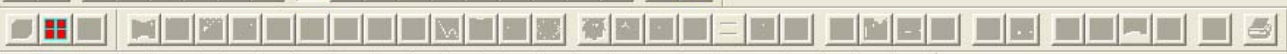

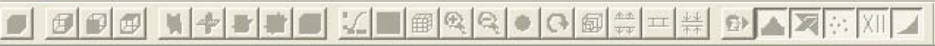

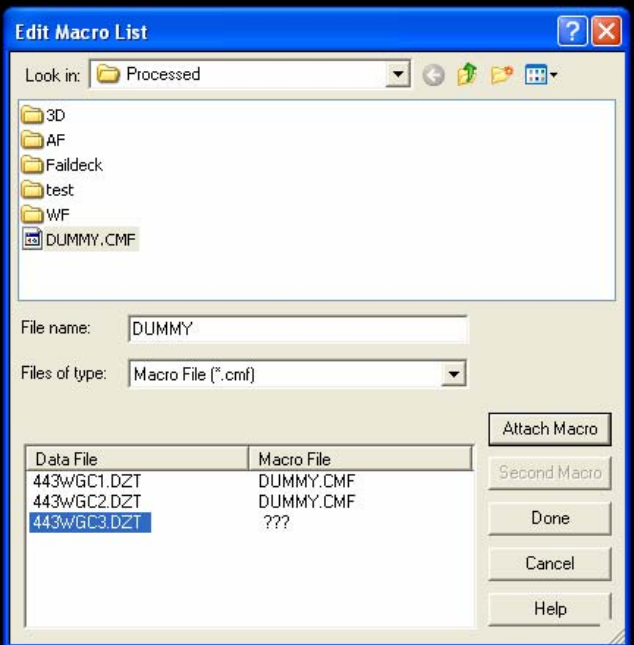

Figure A7: Attach a dummy macro to all files. 
STEP 5: On the ensuing screen, define the actual 3D physical characteristics. Under the "X Max" column, insert the length of the survey (for consistency purposes, an aluminum tape is recommended to be placed on the boundary of the survey area). Insert the distance between scan under the "Y Max" column (Figure A8). Click on "3D Output Filename" and give it any name. The 3D construction will generate a $3 \mathrm{D}$ file by stitching together all the files provided before.

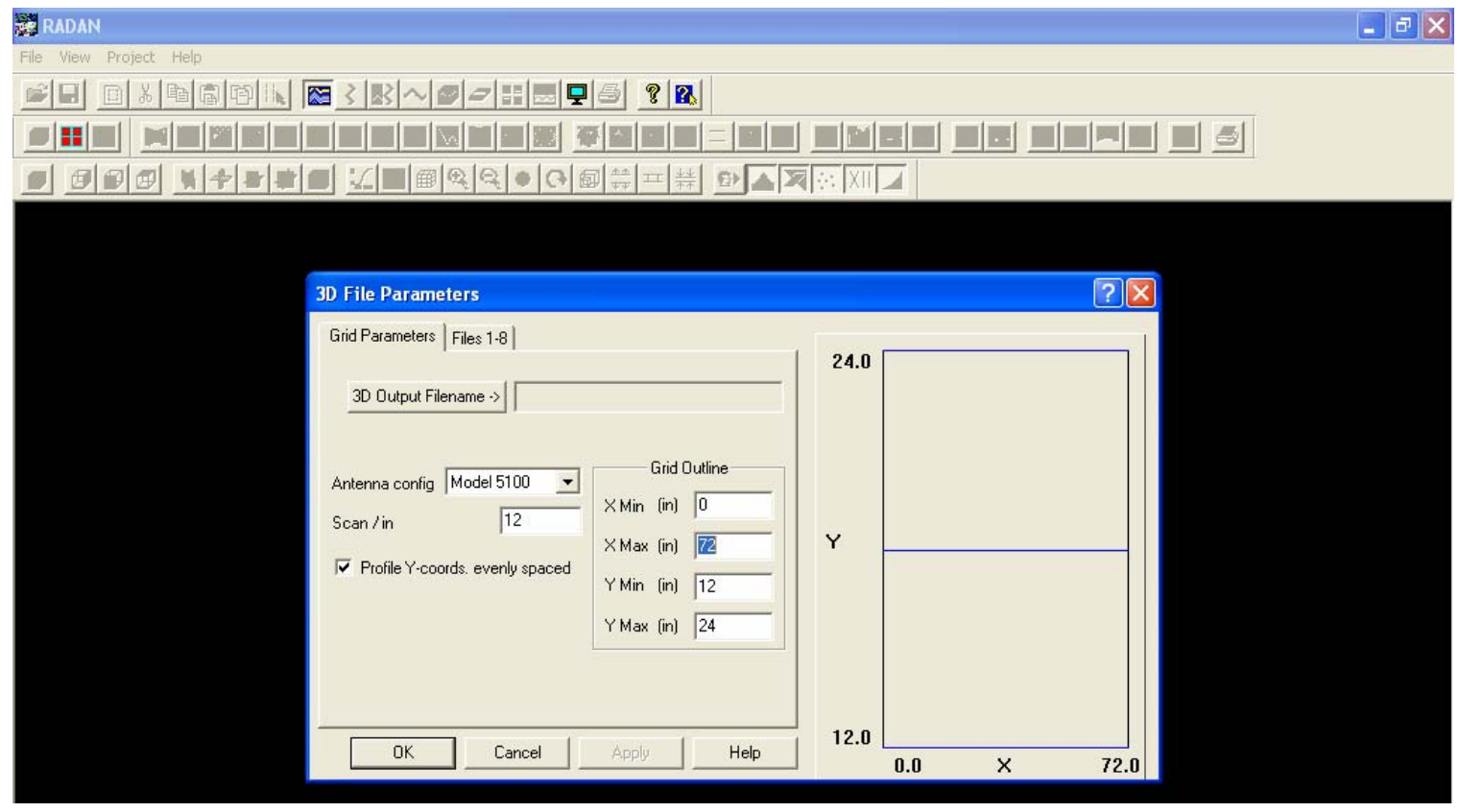

Figure A8: Provide 3D file parameters.

STEP 5: Click on "Files 1-8" tab to select the beginning of the file for each file. If more than 8 files are added, there will be tabs like "Files 9-16" and so on (Figure A9). Click on "Click" to open up the file and manually pick out the beginning of the file. If aluminum tape was used to mark the boundary of the survey area (as shown in the example), the selection of the beginning of file should be easy (Figure A9). Once the beginnings of all the files are picked, click "Apply", then click "OK". 


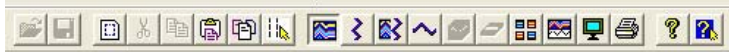

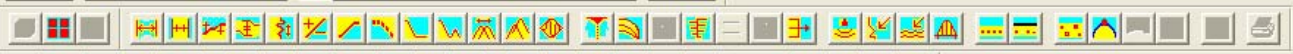

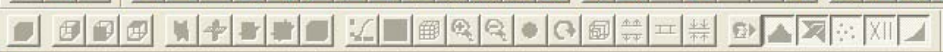

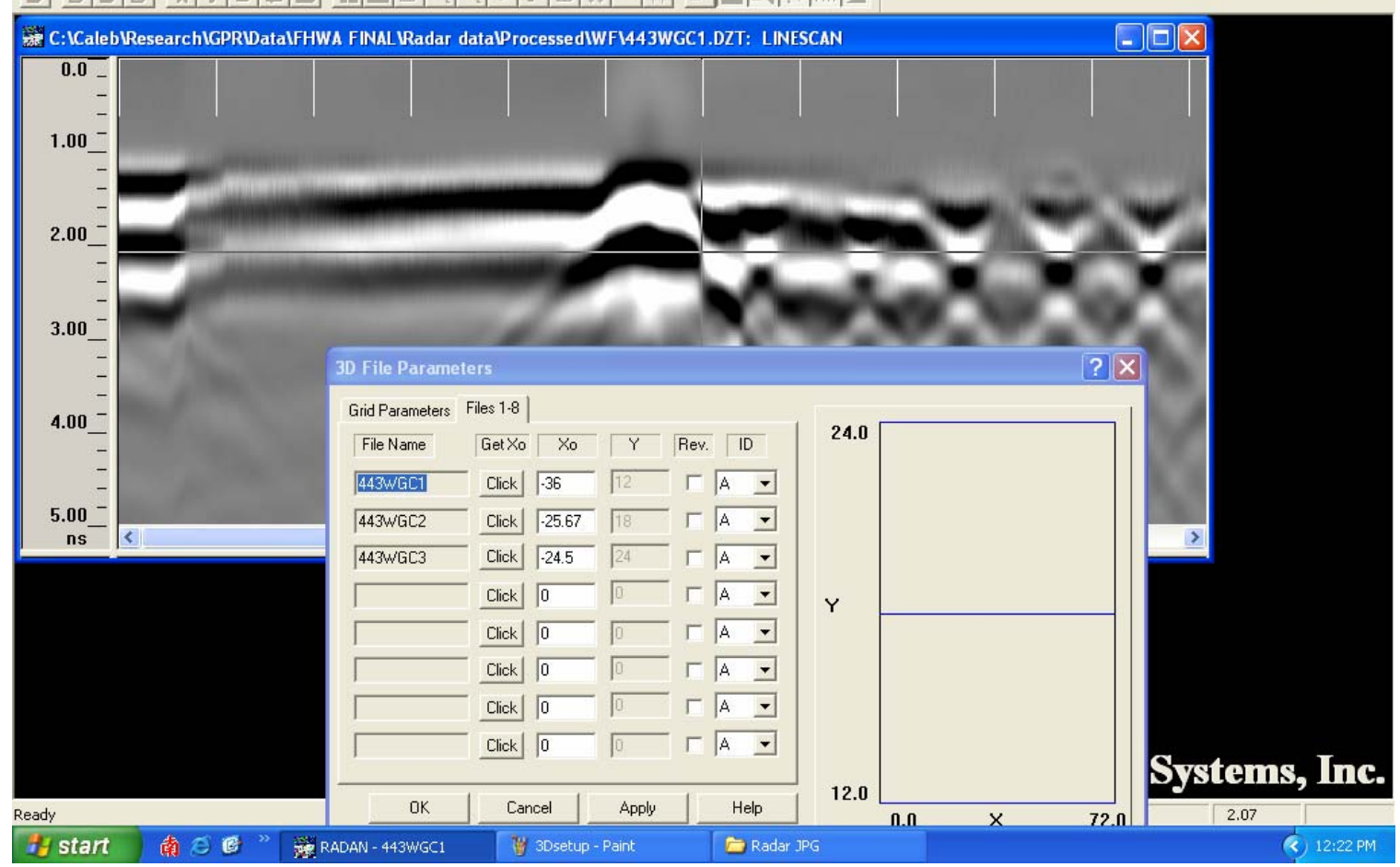

Figure A9: Pick the beginning of each file.

STEP 6: Click "Run" under the "Process" tab to initiate stitching of the files based on the parameters provided.

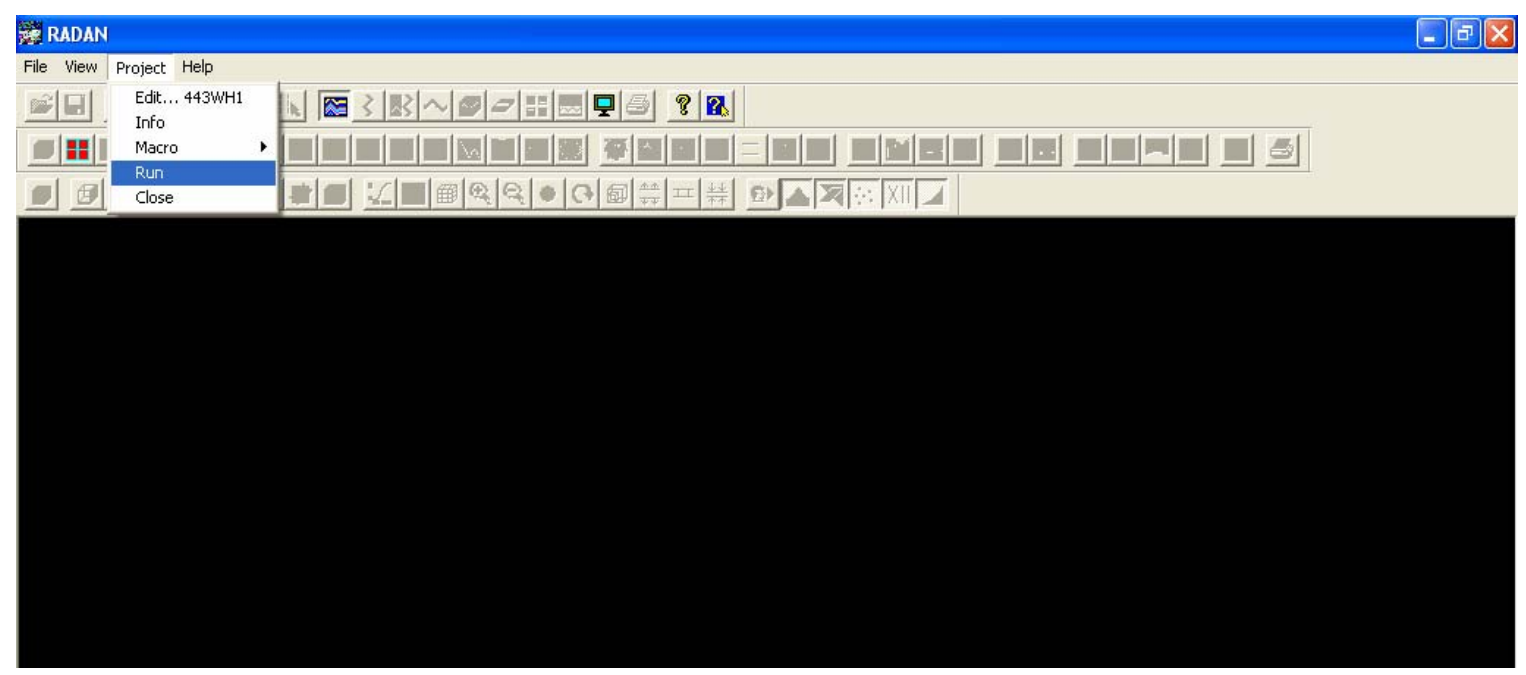

Figure A10: Initiate the stitching of the files. 
STEP 7: Once the process of stitching is complete, a large "stitched" file can be seen (Figure A11). By selecting the "3D Mode", a set of 3D post-processing options will be activated (Figure A12).

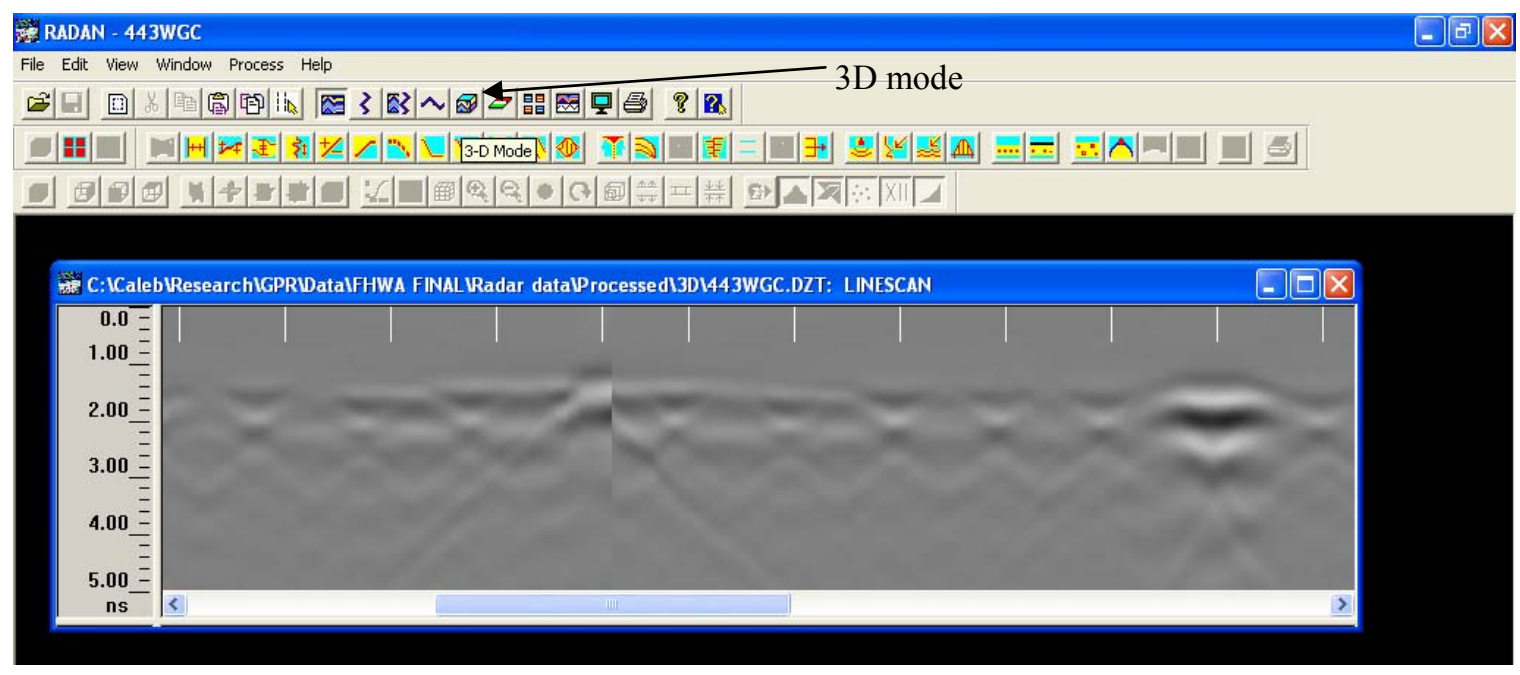

Figure A11: 3D "stitched" file.

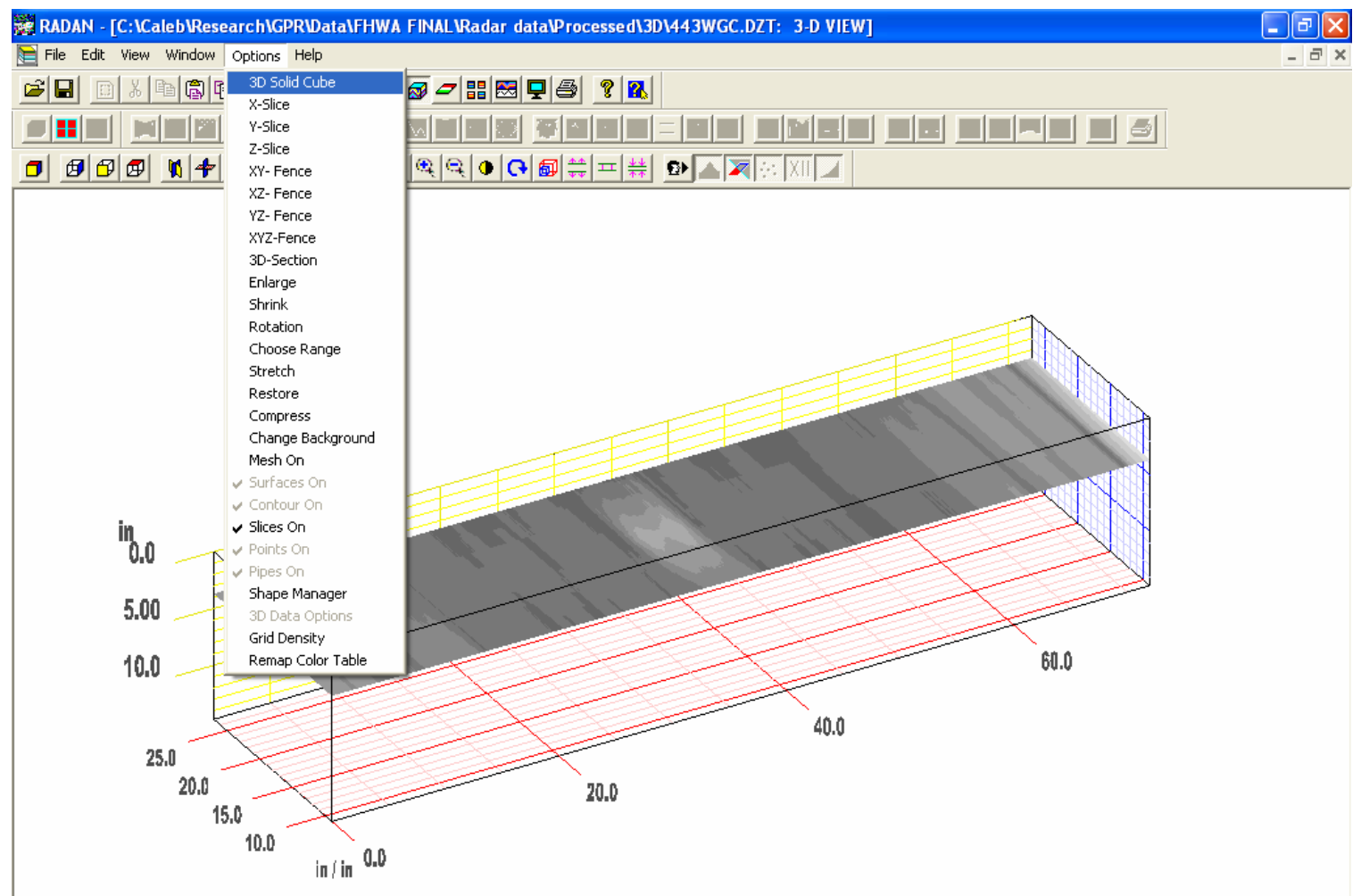

Figure A12: 3D processing options. 